\title{
Introducing War: \\ Visual Analysis of Online Media Discourse on Forces and Weapons of the Iraq Conflict
}

\author{
by \\ George Ross \\ In partial fulfillment of \\ the requirements for the degree of \\ Master of Arts
}

School of Linguistics and Applied Language Studies

Carleton University

(C) George Ross

September 2008 


$\begin{array}{ll}\begin{array}{l}\text { Library and } \\ \text { Archives Canada }\end{array} & \begin{array}{l}\text { Bibliothèque et } \\ \text { Archives Canada }\end{array} \\ \begin{array}{l}\text { Published Heritage } \\ \text { Branch }\end{array} & \begin{array}{l}\text { Direction du } \\ \text { Patrimoine de l'édition }\end{array} \\ \begin{array}{l}\text { 395 Wellington Street } \\ \text { Ottawa ON K1A 0N4 } \\ \text { Canada }\end{array} & \begin{array}{l}\text { 395, rue Wellington } \\ \text { Ottawa ON K1A 0N4 } \\ \text { Canada }\end{array}\end{array}$

Your file Votre référence ISBN: 978-0-494-43490-1

Our file Notre référence

ISBN: 978-0-494-43490-1

NOTICE:

The author has granted a nonexclusive license allowing Library and Archives Canada to reproduce, publish, archive, preserve, conserve, communicate to the public by telecommunication or on the Internet, loan, distribute and sell theses worldwide, for commercial or noncommercial purposes, in microform, paper, electronic and/or any other formats.

The author retains copyright ownership and moral rights in this thesis. Neither the thesis nor substantial extracts from it may be printed or otherwise reproduced without the author's permission.
AVIS:

L'auteur a accordé une licence non exclusive permettant à la Bibliothèque et Archives Canada de reproduire, publier, archiver, sauvegarder, conserver, transmettre au public par télécommunication ou par l'Internet, prêter, distribuer et vendre des thèses partout dans le monde, à des fins commerciales ou autres, sur support microforme, papier, électronique et/ou autres formats.

L'auteur conserve la propriété du droit d'auteur et des droits moraux qui protège cette thèse. $\mathrm{Ni}$ la thèse ni des extraits substantiels de celle-ci ne doivent être imprimés ou autrement reproduits sans son autorisation.
In compliance with the Canadian Privacy Act some supporting forms may have been removed from this thesis.

While these forms may be included in the document page count, their removal does not represent any loss of content from the thesis.
Conformément à la loi canadienne sur la protection de la vie privée, quelques formulaires secondaires ont été enlevés de cette thèse.

Bien que ces formulaires aient inclus dans la pagination, il n'y aura aucun contenu manquant.

\section{Canada}




\begin{abstract}
This study analyzes visual patterns and strategies used within two sets of documents on the weapons and forces of the Iraq conflict, published online by the CNN (1998) and $\mathrm{BBC}$ (2003) respectively to accompany news coverage of the war. Using a functional approach to image analysis and multimodality and referring to Social Semiotics as an overall theoretical approach, all of the illustrations contained within these documents are analyzed, noting patterns of Representational, Orientational and Organizational meanings, and discussing their possible ideological antecedents. A variety of strategies used by $\mathrm{BBC}$ and $\mathrm{CNN}$ to distance the viewers (users) of these documents from suffering and violence, align them with the Coalition's position in the conduct of war, and present aesthetically-appealing images of Coalition forces. The roles of narrative structures, perspective, coding orientations, and relations between images and articles which they accompany are shown to be of particular importance in producing these portrayals and supporting an ideological position which favours the Coalition's interests.
\end{abstract}




\section{Acknowledgements}

It is with much gratitude and relief that I acknowledge the support, help, and encouragement I have received while working on this thesis project. I am especially grateful to my supervisor, Dr. Lynne Young, who guided me from beginning to end in this long process, going above and beyond to accommodate my sometimes-difficult working habits. I am also thankful to my second reader, Dr. Jaffer Sheyholislami for his support, inspiration and advice, and Dr. Mahmoud Eid, Assistant Professor at the Department of Communication, University of Ottawa, for kindly offering his time, attention, and valuable input on such short notice.

I would also like to thank the faculty at the School of Linguistics and Applied Language Studies, Carleton University, who for many years guided me - especially Dr. Graham Smart, Dr. Richard Darville and Dr. Natasha Artemeva, who helped me find my interest in studies of writing and discourse, and gave me so many opportunities to freely explore it and find my own niche within the field. I have been blessed with many great teachers throughout the last 18 years, and nowhere else was this more evident than during my time at Carleton University. I am also happy to say thanks to the many great classmates I have had here over the years, without whom this experience would never have been complete.

In a very difficult year for me, there is no one I owe more to than my family, both immediate and distant, whose faith and support has meant so much. They had been with me all along, and it is with some regret that I dedicate this work to my grandmother Emma Krylova, who wished me the best in this research but would never see me finish it. I would also like to thank the staff and doctors at Ottawa Hospital, as well as my physiotherapist Omar Sahraoui, for the kind and expedient care I received from them when I needed it most.

Finally, I am glad to acknowledge my friends, most of whom are far from me (if only geographically), and come from organizations with silly acronyms such as SUBSIM, \#AMV and TYHMG. Most of all, I would like to thank Kirill Grunichev and Sergei Zavodski, who still keep up after all these years; Aaron Grossman, who kept me happily distracted with his research ideas; and Helen Ye, who saved me from panic over mine so many times. It is thanks to them that I always found new energy to work, and it I wish them all the best in their academic, professional, and personal lives, wherever they take them. 
Table of Contents

1. Introduction

2. Literature Review

2.1 Introduction

6

2.2 Social Semiotics and Critical Discourse Analysis____ 8

2.2.1 Social Semiotics 8

2.2.2 Critical Discourse Analysis__ 11

2.3 Systemic-Functional Grammar___ 14

2.4 Visual and Multimodal Analysis____ 17

2.5 Online Media and CDA___ 24

2.6 Modern Visual Media and CDA__ 28

2.7 Media Discourse in the U.S. War on Iraq___ 33

2.8 Summary __ 36

3. Methodology and Data_ 37

3.1 Methodology - Introduction__ 37

3.2 Functional Analysis: Terminology__ 38

3.2.1 - Adjusted Analytical Framework__ 42

3.3 Ideational / Representational Function__ 43

3.3.1 Narrative Structure______ 43

3.3.1.1 Processes_ 45

3.3.1.2 Vectors__ 46

3.3.1.3 Participants_ 47

3.3.1.4 Circumstances__ 49

3.3.2 Conceptual Structures

3.3.2.1 Classification Structures (Taxonomies)___ 52

3.3.2.2 Analytical Structures__ 53

3.3.2.3 Symbolic Structures _ 56

3.4 Interpersonal / Orientational Function__ 57

3.4.1 Perspective (Attitude)__ 58

3.4.1.1 Size of Frame (Distance)__ 58

3.4.1.2 Horizontal Angle__ 60

3.4.1.3 Vertical Angle_ 61

3.4.1.4 Perspective - Combination___ 62

3.4.1.5 Narrativization 63

3.4.1.6 Perspective in Scientific Images___ 64

3.4.1.7 Gaze_ 65

3.4.1.8 Perspective and Privilege___ 66

3.4.2 Modality _ 67

3.4.2.1 Depth_ 70

3.4.2.2 Detail _ 70

3.4.2.3 Colour 71

3.4.2.4 Lighting 71

3.4.3 Coding Orientation (Modality Configurations)__

3.5 Textual / Organizational Function______ 75

3.5.1 Text-Image Relationship_________ 76

3.5.2 Framing, Salience, Linearity__ 78

3.5.2.1 Framing__ 78

3.5.2.2 Salience__ 79 
3.5.2.3 Linearity 81

3.5.3 Informational value _ 82

3.5.4 Recurrent Patterns_— 84

3.6 Data_ 86

3.6.1 Modern War and the Media___ 86

3.6.2 New Media___ 97

3.6.3 CNN and $\mathrm{BBC}$ - Background _ 100

3.6.4 Data Selection - CNN and BBC Documents___ 101

3.6.5 Data Organization__ 103

\section{Analysis}

3.6.6 Micro-analysis, Narrowing Data Selection___ 105

4.1 Macro-Analysis 107

4.1.1 Genres 107

4.1.1.1 Ordinary Photograph__ 107

4.1.1.2 Publicity Photograph_ 109

4.1.1.3 Press Collage__ 111

4.1.1.4 Scale Drawing 112

4.1.1.5 Function Diagram__ 113

4.1.1.6 Map_ 114

4.1.1.7 Optical Sensor Data__ 115

4.1.1.8 Combination_ 116

4.1.1.9 Summary

4.1.2 Functions 118

4.1.2.1 Primary illustration__ 118

4.1.2.2 Secondary Illustration_ 119

4.1.2.3 Exposition / Explanation__ 119

4.1.2.4 Summary_ 121

4.2 Micro-analysis__ 122

4.3 Section I - Coalition Vehicles__ 122

4.3.1 Organizational —_ 123

4.3.1.1 CNN 124

4.3.1.2 BBC 126

4.3.1.3 Summary _ 128

4.3.2 Representational__ 129

4.3.3 Orientational___ 132

4.3.4 Summary 139

4.3.5 Exceptions 140

4.4 Section II - Coalition Troops___ 143

4.4.1 Image $1 \_144$

4.4.2 Image $2 \longrightarrow 147$

4.4.3 Images 3 and 4

4.4.4 Image $5 \longrightarrow 154$

4.4.5 Image $6 \longrightarrow 156$

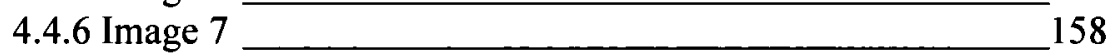

4.4.7 Summary _ 160

4.5 Section III - Iraqi Forces and Equipment___ 162

4.5.1 Troops_ 163

4.5.2 Vehicles__ 167

4.5.3 Summary _ 171

4.6 Section IV - Coalition Weapons___ 171 
4.6.2.1 Topography__ 177

4.6.2.2 Topology 179

4.7 Section V - Biological and Chemical Weapons___ 184

4.8 Summary _ 190

5. Discussion / Conclusion__ 191

5.1 Privileged Positions, Generic Types___ 193

5.2 Coding Orientations, BBC vs. CNN__ 195

5.3 Strategic Patterns _ 197

5.3.1 Alignment__ 198

5.3.2 Distancing — 199

5.3.3 Abstraction 200

5.3.4 Aesthetic Appeal__ 201

5.4 Culture of War and Technology__ 202

5.5 Limitations and Directions for Further Study___ 204

5.6 Summary__ 206

6. References__ 208

7. Appendix I - Sample Images _ 213

8. Appendix II - Sample Images - Analysis__ 221 
War is understood and interpreted, justified and judged through the images and narratives that tell the stories of war. Most civilians experience military conflict through the signs and symbols of its depiction, their impressions derived not from the battles in distant lands but from the manner they are rendered at home.

(Andersen, 2006, xvi)

\section{Introduction}

The present study examines visual aspect of the discourse found in the online documents by BBC (2003) and CNN (1998) which introduce news readers to the weapons and forces of the Iraq conflict. Within these documents, a variety of visual images, both photographic and diagrammatic, are used to cover a range of topics relating to both Coalition and Iraqi forces - in ways that appear diverse, but also highly strategic. To uncover these strategies, a comprehensive methodology for functional analysis of visuals is developed and applied in this study, following from the visual analysis work of O'Toole (1994), Lemke (1998, I999, 2002, 2004, 2005), Oyama and Jewitt (2001), Iedema (2003), Machin (2004), Teo (2004), van Leeuwen (2005), Kress and van Leeuwen (2006), Royce and Bowcher (2007) and Knox (2007). This analysis is applied as part of a Social Semiotics approach, as outlined by Halliday (1978), Hodge and Kress (1988) and van Leeuwen (2005) - drawing also on elements of Critical Discourse Analysis (CDA), as outlined by Fairclough and Wodak (1997), Wodak (2001), van Dijk (2001) and others to offer a critical perspective on the discourse at hand. I take the view that visual choices made by $\mathrm{BBC}$ and $\mathrm{CNN}$ in presenting this highly complex issue are far from neutral, factual representations - but rather reflect dominant ideologies to a significant extent and thereby do important ideological work.

The choice of data - which is visual and originates from an online environment - is, in my view, highly relevant in today's media landscape. The 
Internet is in itself a relatively new semiotic environment - but has become an extremely important media source in the past several years, widely referred to as the contemporary 'new media'. In her 'call to arms' to discourse analysts, Mautner (2005) points out that there has been relatively little CDA research into online media published in journals in the preceding several years, and while this trend is currently changing, there is still a significant unfulfilled potential for research in this area. Due to the technological capabilities of Internet technology, online media has a significantly more complex multimodal potential than traditional print or even television media, and uses images in ways that are likewise different. Kress and van Leeuwen (2006), in their comprehensive guide to functional visual analysis titled Reading Images, state that "The world represented visually on the screens of the "new media' is a differently constructed world to that which had been constructed on the densely printed pages of the print media" (p.31). This study will thus seek to explore ways in which visual resources of online media are being used to present complex issues related to war, and patterns which emerge in the use of these resources. On this point, it is also interesting to observe how these patterns differ between $\mathrm{CNN}$ and $\mathrm{BBC}-$ even from the fact that there is a five-year gap in publication date between the two, during which time Internet technology developed rapidly, revealing the dynamics of change and ways in which the visual potential of online media has been deployed more thoroughly over time.

The primary set of research questions for this comparative study is as follows:

1) How are weapons, forces and actions of war presented?

2) How are images used within BBC (2003) and CNN (1998) special documents on weapons and forces of the U.S. war on Iraq?

3) How is the viewer positioned relative to the war and the weapons in forces with which it is conducted? 
The positioning of these questions in existing theory of visual analysis will be discussed in detail in the following two chapters.

The above questions are, in themselves, relatively neutral and are a starting point for guiding the analysis, however as with any critical study, there are underlying ideological questions guiding this study as well. As Fairclough and Wodak (1997) emphasize, "CDA sees itself not as dispassionate and objective social science, but as engaged and committed. It is a form of intervention in social practice and social relationships" (p.258). In the present study, therefore, I take a critical stance toward the presentation of war, seeking to establish a connection between the representative patterns and their ideological antecedents. This study will show that these antecedents are directly realized in visual discourse. Kress and van Leeuwen (2006), in their seminal work on image analysis, emphasize that "even though the visual mode might seem to provide direct access to the world, it is as amenable to realizing theoretical positions as is the verbal" (p.30). Thus, additional questions to be addressed in this study are as follows:

4) How do visual presentations of weapons by CNN (1998) and BBC (2003) reflect dominant ideologies? What resources are used to create positive evaluations of military technology? How is the viewer aligned with dominant ideological positions, and how is the viewer distanced from negative aspects of the war?

A key starting point for the critical aspect of this study is Chouliaraki's $(2005,2006 a)$ research on BBC television's coverage of the early stages of the U.S. war on Iraq. Chouliaraki (2005) points out that in the immediate lead-up to the U.S. war on Iraq and through the war's initial stages, there was a notable change in British public opinion: "In the case of the war in Iraq, the British public opinion appeared to shift from an anti-war position, registered in polls and demonstrations before March 2003, to a pro-war position as a consequence of the government's "various rhetorical 
devices and a complicit media" (Baines and Worcester 2003, p.16)" (p.144). She stresses however, that "the pro-war bias in western television footage is not a behindthe-scenes co-ordination between government and journalists, but the assumptions already implicit in the routine professional choices that stage and narrate the war in television." (p.144). She outlines a number of ways in which these implicit assumptions manifest themselves, primarily through a pattern of so-called 'aesthetization' - whereby the media presents war as an impressive spectacle to be observed, carried out by inanimate or 'diffused' entities, shown from a safe distance relative to the viewer. In this way, the viewer is simultaneously distanced from actual suffering produced by the war, and prevented from taking an ideological stance against the war itself. While the visual aspect of Chouliaraki's $(2005,2006 a)$ analysis is fairly limited, it does provide some insight into the way media portrayals might operate. The strategy of their application is complex and frequently involves multiple stages. The analysis in this study will thus examine in detail the ways in which these apply to a wide sample of images drawn from the data.

In order to better inform the analysis and understand the ideological and historical processes in which the data is embedded, I also present brief overviews of the recent history of war presentations in the media, drawing on the work by Andersen (2006) and Allan and Zelizer (2004) on the history of war reporting, as well as Lewis' (2007) analysis of the modern American culture of war. These sources provide a critical perspective on the ideologies behind the 'routine professional choices' which Chouliaraki (2005) refers to.

The present study is organized as follows. In Chapter 2, I provide an overview of literature, tracing the origins and principles of Critical Discourse Analysis and the functional approach to Visual Analysis. In Chapter 3, a detailed discussion of the 
functional methodology adopted for the visual analysis is included, drawing on literature described in the preceding chapter, followed by a summary of the data and a description of the context in which the CNN (1998) and BBC (2003) documents were published - both in terms of the 'new media' context in which they are found, and the media discourse of war in general. In Chapter 4, the analytical framework is applied to the data, providing a detailed qualitative description of patterns found in images and image sets, supported by analysis of distribution of images by functions and generic types. In Chapter 5, the findings of the analysis are discussed and considered, implications to existing theory and future research directions suggested, and overall conclusions are drawn. 


\section{Literature Review}

\subsection{Introduction}

The present study is situated in the developing field of visual and multimodal analysis, a currently-developing field which has emerged from research of discourse and society in general. This chapter will examine some of the currently-published work on relevant topics and trace relevant theoretical antecedents for a research methodology, which will be laid out in Chapter 3 .

Firstly, the primary framework(s) of Critical Discourse Analysis (Wodak, 2001; Fairclough and Wodak, 2001; Fairclough, 1992, 2002; van Dijk, 2001; Young and Fitzgerald, 2006) and Social Semiotics (Halliday, 1978; Hodge and Kress, 1988; van Leeuwen, 2005) is briefly explained, with their underlying assumptions examined as relevant to the present study. Developments of analytical theories within these fields as well as their applications are also discussed. One of the primary methodologies used by researchers in Critical Discourse Analysis and Social Semiotics is Systemic-Functional Grammar (Halliday, 1994; Eggins, 2004; Young and Fitzgerald, 2006); the metafunctional approach offered by this method is outlined further below.

Following from and developing out of this approach is the theory of visual analysis and multimodality (Lemke 1998, 2002, 2005; Van Leeuwen and Jewitt, 2001; Kress and van Leeuwen 2001, 2006) that has been developed as an extension of systemic methods from language to visual and other types of non-linguistic communication. The features of this theoretical approach form the core of the methodology for the present study, and are thus discussed in terms of their value toward analysis. 
With the rapid technological and social development of the Internet as a communication medium and alternative mass media source, there have been calls for discourse analysts to work more extensively with online media - including Mautner (2005), who called for Critical Discourse Analysis to 'get wired', and Lemke's (2002, 2004, 2005) work on Hypermodality, Multimedia genres and traversals, which has provided important theoretical grounding for analyzing the non-linear discourse features found on the Internet.

Further, a variety of applications of multimodal analysis approaches to modern media will be discussed, including Knox's (2007) examination of online newspapers, Machin and van Leeuwen's (2005) analysis of computer games as visual and political discourse, van Leeuwen and Jaworski's (2002) study of the discourse of war photography, Teo's (2004) analysis of public poster campaigns in Singapore, as well as Royce's (2007) and Bowcher's (2007) work on multimodal complementarity in media articles on financial issues and rugby players. All of these offer useful theoretical suggestions to consider for developing a visual analysis methodology, and will be briefly summarized and considered in terms their relevance to the present study.

As already noted in the introduction, one of the key starting points for this study is Chouliaraki's $(2005,2006$ a) work on the television news coverage during the initial stages of the 2003 U.S. war on Iraq. The final section of this chapter will examine the findings, approaches and assumptions of Chouliaraki's research on the U.S. war on Iraq in detail, focusing on applications of her findings towards the method taken in this study, which will be discussed in the subsequent chapter. 


\subsection{Social Semiotics and Critical Discourse Analysis}

In this study, I take an approach to communication which originates in Social Semiotics and Critical Discourse Analysis (CDA), two sociolinguistic movements which have developed over the course of the last three decades. Social Semiotics originates in Halliday's (1978) work which considered language as a means for realizing social meaning, rather than a mental code. CDA began as a research circle which used sociolinguistic methods, including those applied by Halliday (1978) to the study of Social Semiotics, but distinguished by its particular concern with applying these methods to uncovering and challenging the ideologies of dominant groups in society (Fairclough and Wodak, 1997). In this section, definitions of these approaches by Hodge and Kress (1988), Fairclough and Wodak (1997), Wodak (2001), van Dijk (2001), Halliday and Mattheissen (2004) and van Leeuwen (2005) will be presented.

\subsubsection{Social Semiotics}

Social Semiotics developed as a reaction to traditional semiotics, which studied meaning in isolation, as a system of codes. The key unit examined by semiotics is the Sign, which is composed of a Signifier (form) and a Signified (meaning) (Halliday, 1978). Traditionally, these were seen to form abstract systems which could be observed in actual linguistic use, as Hodge and Kress (1988) describe: “a system of signs as an abstract structure which is realized or instantiated in text" (p.6) However in social semiotics, as they stress, "every system of signs is the product of processes of semiosis, and documents the history of its own constitution. Terms in a system have value by virtue of their place in that system. At the same time, a system is constantly being reproduced and reconstituted as texts. Otherwise it would cease to exist." (p.6) Social Semiotics is thus concerned not only with the system of signs, but the motivation behind them - whereas traditionally, these were seen as largely 
arbitrary, powerful only by virtue of the meaning attached to them. Social Semiotics emphasizes what Hodge and Kress (1998) termed 'power as meaning' - the notion that any system of meaning is a realization of ideologies produced by social institutions (p.2).

The view of language and grammar adopted by Social Semiotics, as as per Halliday (1978), is a functional one: "grammar is not a code, not a set of rule for producing correct sentences, but a 'resource for making meanings"' (p.192), a system of choices guided by social considerations. Van Leeuwen (2005) suggests that every semiotic resource, including (but not exclusive to) grammar, has a meaning potential which is determined by the way this resource is (or can be) applied in real communicative situations "Studying the semiotic potential of a given semiotic resource is studying how that resource has been, is, and can be used for purposes of communication, it is drawing up an inventory of past and present and maybe also future resources and their uses" (p.5). The semiotic potential of any system is 'inflected' based on the context in which it is applied - as van Leeuwen (2005) suggests, the semiotic choices made by individuals and groups are largely caused cultural differences, differences in sources of authority, and differences in the roles of participants within the same field of practice or institution.

Methodologically, Social Semiotics approaches semiotic choices by constructing 'system networks' - inventories of all possible types of meanings within a system (such aspects of grammar, for instance), and then considers how these are realized and which of the possible choices within the system are made (Halliday, 1978; van Leeuwen, 2005). Possible antecedents of these choices are then discussed. Within any group, institution or ideology, these antecedents could be expressed as sets of rules (which are generally tacit) governing ways in which semiotic choices can be 
made. Van Leeuwen (2005) recommends approaching sets of choices within communicative contexts with the following questions in mind:

1. How is control exercised, and by whom?

2. How is it justified?

3. How strict are the rules? How much room do they allow for individuality and difference?

4. What happens when people do not follow the rule? What sanctions are attached to deviance?

5. Can rules be changed, and if so, in what way, and for what kinds of reasons? (p.53)

Social Semiotics is thus concerned with ways in which institutions and ideologies restrict or enable semiotic choices available through the use of grammatical and other resources. This approach sees semiotic rules as being grounded in a set of 'ideological complexes', defined by Hodge and Kress (1988) as follows:

a functionally related set of contradictory versions of the world, coercively imposed by one social group on another on behalf of its own distinctive interests or subversively offered by another social group in attempts at resistance in its own interests. An ideological complex exists to sustain relationships of both power and solidarity (p.3)

Ideological complexes, under this perspective, are composed of relational and actional models of society - the former being a classification of society into different kinds of 'social agents,' and the latter being responsible for specifying the types of actions and behaviours 'required, permitted or forbidden' to these types of 'social agents' (p.3). Social order is maintained through discourse, which Hodge and Kress (1988) define as "the social process in which texts are embedded" (p.6) - with 'text' in this case being defined as "the concrete material object produced in discourse" (p.6). Powerful texts are those which, by virtue of being produced through ideological complexes, attain 'institutionalized legitimacy and authority' (p.9).

While discursive power held by institution has significant social effects, it is not static. Just as society is dynamic by nature and social power can be negotiated and 
challenged, semiotic systems which produce texts constantly undergo change through negotiation:

Meaning is always negotiated in the semiotic process, never simply imposed inexorably from above by an omnipotent author through an absolute code. [...] Social semiotics cannot assume that texts produce exactly the meanings and effects that their authors hope for: it is precisely the struggles and their uncertain outcomes that must be studied at the level of social action, and their effects in the production of meaning. (p.12)

Contrary to the focus of traditional semiotics on constant, arbitrary systems of signs, Social Semiotics is directly concerned with processes of social change and seeks to examine systems of signs in parallel to these social processes. It relies on models of context in explaining communication, and stresses the direct relationship between discourse and ideology - "every semiotic act has an ideological content" (p.40).

For the purposes of the present study, the notion of system networks and rules which determine choices made within them is methodologically important, and informs the functional network for visual analysis outlined in Chapter 3. The suggestion that these choices are motivated ideologically is also highly relevant, and will be discussed further below.

\subsubsection{Critical Discourse Analysis}

Critical Discourse Analysis emerged as a network of scholars in the early 1990s (Wodak, 2001, p.4), including figures such as Ruth Wodak, Teun van Dijk, and Norman Fairclough. Key features of this movement include its concern with notions of power, ideology, history and social context in the study of linguistic communication, at least initially. Fairclough and Wodak (1997) define the approach of CDA as follows: "Critical discourse analysis [...] analyses real and often extended instances of social interaction that take a linguistic, or a partially linguistic form. The critical approach is distinctive in its view of (a) the relationship between language and society, and (b) the relationship between analysis and the practices analyzed" (p.258). 
It should be noted that CDA and Social Semiotics, as understood in this

study, are not mutually exclusive approaches, and CDA is in many ways a development of Social Semiotics oriented towards a more specific, critical purpose. The crucial distinction for CDA lies in its orientation towards not merely observation and analysis of systems of meaning and their ideological antecedents, but social action through criticism - as van Dijk (2001) stresses, "It focuses on social problems, and especially on the role of discourse in the production and reproduction of power abuse or domination. Wherever possible, it does so from a perspective that is consistent with the best interests of dominated groups" (p.96). Research in CDA is generally preoccupied with uncovering injustices and exposing ideological positions; and van Dijk's stance on CDA is certainly telling of the field's overall orientation toward active criticism - he dubs the approach "discourse analysis "with an attitude"" (p.96) and states that instead of maintaining scientific neutrality, it takes positions and argues them through analysis - and does so with enthusiasm, if his own stance is any indication: "CDA is biased - and proud of it" (p.96). Other theorists such as Fairclough and Wodak, while stopping short of professing bias in such direct terms, certainly suggest a similar approach: "CDA sees itself not as dispassionate and objective social science, but as engaged and committed. It is a form of intervention in social practice and social relationships" (p.258).

Within CDA, there are many different methodologies - as van Dijk (2001) is careful to point out, "CDA does not provide a ready-made, how-to-do approach to social analysis, but emphasizes that for each study a thorough theoretical analysis of a social issue must be made, so as to be able to select which discourse and social structures to analyze" (p.98). He suggests that the methodology of CDA should be driven primarily by the demands of the data it studies, and the context from which this 
data originates - "in CDA, we must make choices, and select those structures for closer analysis that are relevant for the study of a social issue. This requires at least some informal ideas about text-context links that tell us which properties of discourse may vary as a function of which social structures" (p.99).

CDA is concerned with both macro-analysis of ideologically-driven discursive structures and micro-analysis of their linguistic realizations, as Fairclough and Wodak (1997) suggest: "Critical discourse analysis is very much about making connections between social and cultural structures and processes on the one hand, and properties of text on the other. But these connections are rather complex, and are best seen as indirect or "mediated"' (p.277). Even among the theorists cited in this section, however, specific methods and concerns within CDA differ greatly - for instance, Wodak is especially concerned with history as a determining factor in modern antiSemitic discourse (Fairclough and Wodak, 1997); while Fairclough traces specific linguistic structures involved in broad social and political tendencies, for instance as in his work on marketization (1993) and technologization (1996).

Public news media is a traditional 'target' for CDA research, being a highly powerful institution - as Fairclough and Wodak (1997) stress, "key areas of social life are becoming increasingly centred on the media, especially television" (p.259) With the emergence of 'new media' and significant growth in Internet communication generally in the decade since the above quote was published, one can certainly say that this trend has continued, although television is now in the process of being supplanted by a no less pervasive and powerful medium. The effect of the growth of public media, Fairclough and Wodak (1997) suggest, is that institutions of power have increasingly mobilized in their efforts to influence public discourse: "the increased importance of language in social life has led to a greater level of conscious 
intervention to control and shape language practices in accordance with economic, political and institutional objectives" (p.259). Thus, CDA research on media discourse and the institutions of power remains highly relevant. A multitude of researchers continue to operate within CDA and Social Semiotics, working with topics including political discourse (Chilton, 2004), war and the media (Chouliaraki, 2005, 2006a), discourses of science (Halliday and Martin, 1993), and many others cited elsewhere within this study.

The methodology of the present study originates to a large extent in the applications Social Semiotics approaches to visual media, namely those by van Leeuwen (2005), Kress and van Leeuwen (2006), Lemke (1998, 2002, 2004) and O'Toole (1994). The research aims of this study, meanwhile, are influenced by CDA and its concern with uncovering the mechanisms of media discourse and ways in which it frequently seeks to maintain status quo relative to powerful groups and institutions (such as the military and government) within society. I take a critical attitude towards these institutions and seek to uncover the means by which their ideological aims are realized in media discourse.

\subsection{Systemic-Functional Grammar}

As van Dijk (2001) suggests, CDA does not prescribe a specific methodology for conducting analysis - however there are certainly methodologies that are highly suitable to this purpose. Young and Fitzgerald (2004) note that "Critical discourse analysts use several different methods to help them 'dig' beneath the surface of the discourse to answer their questions. One widely used theoretical framework is Systemic Functional Linguistics or SFL - a way of understanding the functions that language performs and the choices people make when they speak/write" (p.16) 
Systemic Functional theory originates from Halliday's work originally published in the 1970s (Halliday and Mattheissen, 2004). SFL, which essentially presents a set of grammatical resources for realizing semiotic choices, forms the methodological basis for Halliday's (1978) original definition of Social Semiotics. The Social Semiotic approach to notions such as ideology, power, and sets of social rules and norms compliments SFL's strictly grammatical focus, helping to explain rationale behind grammatical choices. Eggins (2004) summarizes the underlying questions asked by Systemic-Functional Linguistics (SFL) as follows: "how do people use language? [...] how is language structured for use? [...] how many different sorts of meanings do we use language to make? [...] how is language organized to make meanings?"' (p.3) Halliday's theory is organized around a series of semantic strata, the widest of which is the dimension of Genre, which refers to the overall cultural context and all the meanings possible within a particular cultural context (Halliday and Mattheissen, 2004). Beneath this is the level of Register, which refers to a particular situation within this cultural context. Every situation consists of three dimensions, which are termed Field (topic or focus of activity), Tenor (relations of power and solidarity) and Mode (role of language in structuring the situation), which are understood by readers intuitively and have "significant and predictable impacts on language use" (Eggins, 2004, p.9). Field, Tenor and Mode are mediated through three semantic metafunctions (types of meaning): Ideational, Interpersonal and Textual. In language, each of these is realized through specific lexico-grammatical systems within the text: Ideational meanings through the system of Transitivity - which includes processes (realized by verbs), participants (realized by nouns and pronouns) and circumstances (realized by prepositional and adverbial phrases); Interpersonal meanings through systems of mood (e.g. imperative, interrogative, declarative structures) and modality (e.g. 
certainty, probability, obligation, inclination - expressed through modal verbs and adverbs); and Textual meanings through cohesion (e.g. patterns of reference) and coherence (e.g. thematic structure) (Eggins, 2004; Halliday and Mattheissen, 2004). The diagram below summarizes the structure of this system:

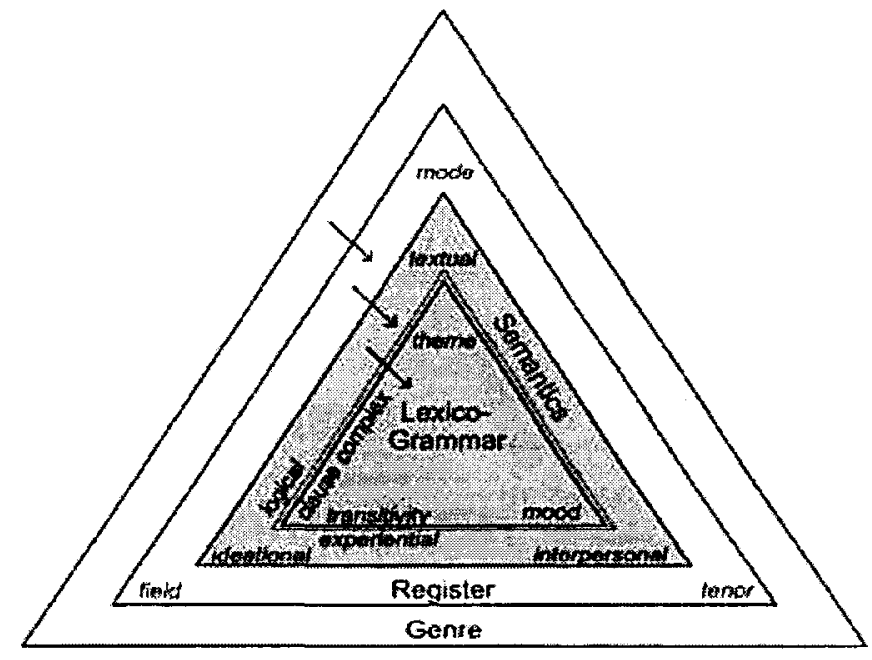

(Eggins, 2004, p.112)

SFL treats lexico-grammatical systems in language as "a finite set of choices or oppositions" (Eggins, 2004, p.13). These choices are taken as both discrete and deliberate, motivated by ideological considerations.

SFL provides an exhaustive set of methodological tools for classifying text features in terms of their functions, and connecting them to higher-level structures. Its strength lies in the fine level of detail and specificity it can provide in micro-analysis. The notion of grammar as a system of choices is an extremely useful one, and is the reason SFL and CDA work well in combination; as Young and Fitzgerald (2006) suggest, whereas SFL is concerned with the 'what' and 'how' of grammatical choices, CDA helps account for the 'why'. Meanwhile the criticisms and observations CDA makes about any type of text are significantly strengthened by SFL's grammatical micro-analytical approach. 
Since the present study examines visual rather than verbal data, SFL does not apply directly to the analysis, however is used to inform it to a significant extent. This is in part due to the fact that visual analysis approaches (O'Toole, 1994; Lemke, 1998, 2002, 2004, 2005; Kress and van Leeuwen, 2006) have generally adapted the assumptions and key structures of SFL analysis to visuals. The same trifunctional approach (i.e. composed of three metafunctions) based on Halliday's (1978; Halliday and Mattheissen, 2004) work is retained within these methodologies, as are many of the concepts - such as participants, processes, circumstances, modality, coherence and cohesion, which were noted above. These are expressed differently in visuals: for instance in language, processes are expressed through verbs, whereas in visuals these rely largely on the system vectors. Useful parallels can, however, be drawn - and these are certainly of greatest value to multimodal studies which consider both visual and linguistic meanings.

The functional approach to visual analysis will be outlined further below.

\subsection{Visual and Multimodal Analysis}

As the discussion above suggests, Social Semiotics (and in fact Semiotics in general) was originally developed based largely on language as the primary communication mode. Halliday (1978) outlined the Social Semiotics approach in reference to the theory of Systemic Functional Linguistics. Much of the work in the field continues to focus primarily on linguistic analysis. Indeed, language and especially the printed word have been the privileged semiotic modality in Western culture for centuries. In traditional semiotics, as Teo (2004) suggests, visuals have been largely ignored, in part due to the perception that their interpretations depended more on their linguistic context and viewers' subjective perceptions than any definite semantic properties: 
"Traditionally, the visual sign has been seen as subordinate to the verbal message [...] Barthes (1977) argued that the meaning of images (and other semiotic codes) is always related to and, in a sense, dependent on verbal text [...] too 'polysemous' and indefinite by themselves $[\ldots]$ and to properly understand them, language has to come to the rescue" (p.193). However, as Teo (2006) argues, approaches based on Social Semiotics have shown visuals to be far from an 'impoverished' system of meaning. Lemke (2005) supports this notion, stating that visual structure and multimodality have always played key roles in text: "Every written genre has always been multimodal, deploying not only the signs of the linguistic system but also those of the visual-spatial meaning systems associated with orthography, typography, and page layout" (p.45). As Kress and van Leeuwen (2006) suggest in their work on visual analysis, Reading Images, technological and social changes in recent times have allowed visual semiotics to challenge the semiotic dominance of language by allowing visuals to be composed and distributed more efficiently than ever before:

The place, use, function and valuation of language in public communication is changing. It is moving from its former, unchallenged role as the mode of communication, to one mode among others, to the function, for instance, of being a mode for comment, for ratification, or for labeling, albeit more so in some domains than others, and more rapidly in some areas than others. (p.36)

They also describe the nature of multimodal relations (i.e. featuring more than one semiotic mode) between visual and text: "the visual component of a text is an independently organized and structured message, connected with the verbal text, but in no way dependent on it - and similarly the other way around" (p.18). With rapid pace of technological development allowing ever greater amounts of visual content to be easily accessible to the public, it is simply no longer justifiable to focus on linguistic communication in the study of discourse. 
In recent years, theorists working in the areas of Critical Discourse Analysis, Social Semiotics, and Systemic Functional Linguistics have taken note of this semiotic shift. There have been calls for greater emphasis to be placed on developing tools capable of accounting for meaning outside strictly linguistics contexts. Lemke's (1999) work on multimodality in scientific discourse stresses the need for the development of such methodologies:

Organizational discourse [...] cannot be abstracted from the medium in which it is materially mediated, nor can it be analyzed solely by tools designed purely for work with extended, connected verbal text. We need analytical tools that are well-adapted for such genres as tables, lists, and charts; for graphicalvisual combinations of other semiotics with verbal text; and for hypertext modes of discourse organization. (pp.44-45)

Lemke $(1998,1999,2002,2004,2005)$ has pioneered much of this work, extending some of the notions associated with multimodality to non-linear 'hypermedia' (2002, 2004) in online environments, and the semiotic structure of traversals (2005) - paths taken by viewers and users of internet multimedia across not merely visual-verbal combinations, but much more complex interactions of multiple types of media. Some of Lemke's work on extensions of multimodality will be considered further below in this chapter.

In terms of visual analysis as such, one of the first and most influential works to employ a functional perspective to analysing images was O'Toole's (I994) The Language of Displayed Art. O'Toole adapted a variation of the same trifunctional framework (referring to three semantic metafunctions) as originally used by Halliday to construct his Systemic Functional Theory. Drawing in part on theories of visual art (and thus introducing concepts such as the Gestalt), O'Toole's framework is detailed to a similar level of detail as SFL, thus proving highly effective and providing a starting point for other theorists to build on. 
By far the most significant development in functional analysis of visuals is Kress and van Leeuwen's (2006) Reading Images, the first edition of which was published in 1996 and drew heavily on O'Toole (1994) while having a significantly broader focus - whereas O'Toole's methodology is geared primarily toward visual art, Reading Images outlined one which is much more universally applicable, something that is achieved in part by a more diverse use of illustrations and analysis examples this book provides. Having been significantly updated for the second edition, this work forms the core of the methodology for the present study - in large part because its organization by metafunctions (ideational, interpersonal, textual) lends itself readily to comprehensive application in a functional study, and allows parallels to linguistics structure to be drawn efficiently from SFL handbooks (Eggins, 2004; Halliday and Mattheissen, 2004) which are organized in a similar fashion. The analytical framework outlined in Reading Images is complemented effectively by van Leeuwen's (2005) Introducing Social Semiotics, which explicitly connects visual analysis to the theory of Social Semiotics. Van Leeuwen (2005) also provides indepth detail on a number of the systems within visuals, particularly in terms of cohesive devices.

Other work on functional approaches visual analysis published in recent years and referred to in this study includes Lemke (1998, 1999, 2002, 2004, 2005), Jewitt and Oyama (2001), van Leeuwen and Jewitt (2001), Machin (2004), Royce and Bowcher (2007), as well as a number of studies which use methodological approaches based on the above works.

A detailed methodology of visual analysis will be presented in the following chapter. The rest of this section, meanwhile, will consider the relationship between 
visual semiotics and previously-outlined approaches to linguistic analysis, as well as discuss some of the aspects of multimodality.

Arguably, the functional framework of visual analysis (as per O'Toole, 1994; Kress and van Leeuwen, 2006) fills precisely the same space as functional grammar. Jewitt and Oyama (2001) emphasize that just as SFL includes a variety of specific grammatical constructs performing specific functions, "Visual social semiotics is functionalist in the sense that it sees visual resources as having been developed to do specific kinds of semiotic work" (p.140). They also note that visual semiotics realizes three metafunctions, much in the same way as text. If we insert this framework into Halliday's model of discourse (see diagram from Eggins (2004), above), the overlap suggests that a functional methodology of visual analysis essentially accounts for the same concepts - with field, tenor and mode being realized through (adjusted) ideational, interpersonal and textual metafunctions, which in turn are realized with specific sets of visual constructs, which perform very similar roles to grammatical constructs outlined in Halliday and Mattheissen (2004) and Eggins (2004). These constructs include Narrative and Conceptual structures responsible for Representational meaning; Perspective and Modality realizing Orientational meaning; and systems of Framing, Salience and lnformation Structure being responsible for cohesion (Kress and van Leeuwen, 2006). Parallels between functional accounts of linguistic grammar and visual structure are very useful to be aware of, and can be used to great effect in multimodal analysis, which considers both text and visuals. While this study is primarily visual, these parallels will still be drawn where applicable in Methodology (Chapter 3) and Analysis (Chapter 4).

Returning to the concept of multimodality, Kress and van Leeuwen (2006) characterize the relationship between semiotic modes in modern media as follows, 
contrasting it to a more traditional language-centred approach: "This incessant process of 'translation', or 'transcoding' - transduction - between a range of semiotic modes represents, we suggest, a better, a more adequate understanding of representation and communication [...] language is not at the centre" (p.39). Bowcher (2007) suggests that multimodal texts are neither redundant nor have visual elements in a dependent role - concluding that their relationship with linguistic aspects of the text is complementary and productive: "although redundancy of meaning may be the result of the simultaneous use of several modes, we cannot say that the resulting meanings that are produced by a multimodal text could be produced if only one mode were to be used" (p.242). Royce (2007) outlines a wide variety of ways in which 'intersemiotic complementarity' (i.e. image-text relations) function - including both synonymous structures where the meanings made in each of the modalities reinforce those made in the other, and conversely antonymous structures where opposite meanings are made in text and visual elements, largely for ironic or humorous effect (p.68). Royce (2007) also suggests that the verbal-visual relations can be used to reinforce attitudes towards certain elements of the discourse (p.98), attract viewer's attention to articles or particular parts of them - serving as a 'stabilizer of weight' (p.99), and suggest potential reading paths for the viewers/readers to take (p.102). Lemke (2005), in his discussion of online media, suggests that images and text are used in parallel and constantly refer to each other, where conventions and overt cues "indicate to a reader (or compositor) of the main text when an image or figure becomes relevant" (p.47), thus alternating the viewer's attention between text and image, reinforcing the meanings made in both. Chouliaraki's (2005) findings also indicate that different modes contained within the same space (in the case of her study - visuals and verbalizations in TV broadcasts) can be used in a complimentary fashion - with one 
modality (visual in the case of said study) taking an active role of narration, explanation or evaluation, while the other modality uses passive exposition to illustrate the narrative.

What is evident from the above observations is that multimodal relationships can function in a great variety of ways and to varying degrees of complexity, while the semiotic load of any multimodal text is generally shared between different modalities - through alternation, juxtaposition, and complementary use. The present study will certainly consider the role of images within the articles of which they are part of, and outline the typical functions they perform.

With increasing use of visuals in modern Western media, the range of meanings that visuals can represent is increasing. As Kress and van Leeuwen (2006) note, images seen in public discourse are becoming 'increasingly coded' - that is, more and more conceptual representations are being embedded into them. Machin (2004), in his study of image banks used by the media, notes that even a relatively naturalistic modality such as photographs is frequently used to represent abstract concepts and idealized, readily-recognizable types of people, objects and events. Machin (2004) suggests that this is in many ways a consequence of social and economic tendencies in society - where it is desirable for 'consumer categories' to be recognizable (p.334). Ways in which this typification and coding is achieved will be discussed further in Methodology (Chapter 3) and Analysis (Chapter 4).

Thus, in the relatively new field of visual semiotics, a fairly comprehensive theoretical and methodological framework already exists and should be applied, tested, and adjusted through practical research, of which there is still only a limited amount, with many aspects of visual discourse in the public context and in relation to institutions of power yet to be discussed. This framework fits well with the overall 
approaches of Social Semiotics and CDA, and is further enhanced by direct use of extensions from SFL. It will thus be adapted as a primary methodology for the present study, and explained in detail in Chapter 3.

\subsection{Online Media and CDA}

As the data used in the present study is drawn completely and deliberately from online sources, the importance of which in the modern media landscape will be later noted in the Background section, one should consider the extent to which such research is relevant and needed in the context of Critical Discourse Analysis.

According to Mautner (2005), there is both significant need and ample cause for discourse analysts to use data from online sources. While the situation has changed somewhat in the past three years, as of 2005 Mautner noted a relative lack of research of online media by scholars working with CDA, as suggested by the number of publications on these topics appearing in CDA journals. She notes that this is not the case in other linguistic disciplines - including corpus linguistics, computational linguistics, and computation studies where online data has been used extensively (p.810). The same is true of communication studies - "ever since the internet 'took off in the mid-1990s, there has been a growing body of research into various aspects of communication on and through the internet. The areas covered include semiotic, textual and interactive properties of computer-mediated communication" (p.810). However the portion of research in CDA which has used online data has remained relatively low-although there are notable exceptions, in particular the work of Lemke $(2002,2004,2005)$ which laid significant groundwork for further research into online, multimodal semiotics. His work in this field will be discussed further below. 
Thus Mautner (2005), in effect, issues a 'call to arms' for critical researchers. Aside from the prospect of using new, previously unresearched data, ease of processing and the sheer amount of discourse to be found online, Mautner also notes aspects like 'diversity of voices' for those interested in interested in 'emancipatory agendas' (p.816), a dynamic, non-linear structure and frequently interactive of the discourse itself (p.819) which is not found in any other medium, and multimodal potential (p.820). Lemke (2005) suggests that the online environment also affords unique opportunities for observing genre development, since the structure of online media accelerates this development significantly: "Hypertext, especially open-ended hypertext such as the WorldWideWeb, affords great opportunity for the emergence of new genres. [...] Genre evolution, or at least the emergence of new genres, is likely to occur much more quickly under these conditions" (p.53).

There is certainly a need in the field for this type of research. Mautner's findings aside, in the course of my own research I had not found any studies similar to my own in terms of data and approaches used to analyze it, with the possible exception of Knox (2007), whose study of online newspapers, while slightly more oriented towards text and multimodal relations (rather than strictly visual semiotics), certainly parallels the present study in some ways. Knox examines both visual and verbal aspects of the front pages of three online newspapers; his visual methodology is based to a large extent on O'Toole's (1994) and work, as well as Kress and van Leeuwen's (2006) Reading Images - however what he does with it is substantially different from my aims in the present study, as he is mostly concerned with page layouts rather than illustrations. Rather than focusing on representational and orientational meanings (as I do), he is primarily concerned with the organization of the websites, both visually and verbally. He notes the predominance of highly 
condensed types of texts, which he defines as "newsbites", and discusses at length the non-linear organization of the discourse within these pages. Knox (2007) suggests that among online newspapers, there is significant consistency developing in terms of design, as his study "shows how digital, discursive and ideological convergences are contributing to the development of a grammar of visual design in English-language online newspapers which crosses national and cultural boundaries." (Knox, 2007, p.20). The most primary similarities are in terms of organizational meanings, particularly the layout which allows viewers to skim more efficiently and traverse the pages in a primarily non-linear fashion. In this latter discussion, Knox relies heavily on Lemke's (2002) article Travels in Hypermodality, one of the most prominent works dealing with online discourse in terms of Social Semiotics.

Lemke (2002) provides the following definition for the term he introduces to describe the modality of online discourse, referring to the concept of 'hypertext', which describes the non-linear nature of text structures online: "Hypermodality is the conflation of multimodality and hypertextuality. Not only do we have linkages among text units of various scales, but we have linkages among text units, visual elements, and sound units. And these go beyond the default conventions of traditional multimodal genres" (p.302). He stresses the non-linearity of hypertext as a salient feature of online communication - "There are many possible trajectories, or traversals, through the web of a hypertext" (p.301). One of the most important aspects of Lemke's (2002) study is that he stresses the role of multimodal and visual semiotics in designing a methodology to study any online discourse: "I propose here that one useful way to understand the design resources afforded by hypermodality is to consider multiplicative combinations of the presentational, orientational, and 
organizational resources of each semiotic mode (language, depiction/ imagery/graphics, and soundforms) (p.301).

Lemke's findings regarding hypermedia are further extended in his (2004) and (2005) work, where he focuses on the idea of traversals even more extensively, suggesting that these traversals cross more than just boundaries of verbal and visual modalities of hypertext in a non-linear fashion - they cross the much more fundamental boundaries between sites, institutions, and roles. Lemke (2005) suggests that current technology allows people to simultaneously function in multiple contexts: "In addition to hypertext, the notion of traversal also applies, I believe, to analyses of how we increasingly cycle our attention among various immediate and virtual worlds" (p.54). This, in his view, has potentially major effects on the role of dominant institutions in defining the identity and activity of individuals in society, since traversals now allow individuals to freely cross institutional boundaries, associating and participating in activities far outside their immediate physical context, and using virtual worlds and games to interactively explore roles and identities which they would never have access to in their real-life institutional context (Lemke, 2004, 2005). While traversal on this level is beyond the scope of the present study, this is certainly a promising direction for further research into hypermedia. In terms of portrayals of war, traversals between news media and entertainment would be interesting to examine - and indeed, research on both already exists (see discussion of Machin and van Leeuwen (2005), further below).

As work by Mautner (2005), Lemke $(2002,2004,2005)$ and Knox (2007) suggests, online media is a rapidly developing discourse environment with rich multimodal and visual resources available for analysis. With rapidly developing technologies, its multimodal capabilities are only increasing. Mautner (2005) has 
outlined notable gaps in terms of CDA research into this type of media, some of which are yet to be filled. Also of interest is the non-linear potential of the Internet which, at the very least, represents a radically new way of reading/using media such as news (Knox, 2007), and at most - signals a significant social change in the role of institutions and the position of individuals relative to them (Lemke, 2004, 2005). The present study thus aims to address some of the unique aspects of online discourse, considering them in relation to the visual content at the centre of this study.

\subsection{Modern Visual Media and CDA}

There are a number of published studies of modern visual media, some of which have already been mentioned above; for the remaining part of this chapter, however, I will focus on four in particular - Machin and van Leeuwen's (2005) examination and comparison of the Black Hawk Down movie and video game, van Leeuwen and Jaworski's (2002) comparison of photographs relating to the Israeli-Palestinian conflict in British and Polish newspapers, Machin's (2004) study of image banks, Teo's (2004) study of national campaign posters in Singapore and Chouliaraki's $(2005,2006 a)$ work on TV reports from the early stages of the 2003 U.S. war on Iraq. The latter, being of core importance as a starting point for the present study, will be discussed in the next subsection, while this section will deal with the former two.

Machin and van Leeuwen's (2005) study looks at Black Hawk Down, an American war film and video game of same name, both of which were developed with assistance from US military. The findings emphasize the role of perspective, agency and identity in reframing the events of the 1993 conflict in Somalia. The viewer (or in the case of the video game, player) is aligned with the American position against Somali warlords, and distanced from suffering, especially of civilians, through a number of devices the most important of which is use of identity. Both the 
movie and the game are noted for their use of 'collectivized', rather than individual identity, with most participants being shown as representatives of groups rather than individuals - which are visually characterized through appearance and attributes, and verbally described in terms such as 'elite soliders', 'operators', 'civilians', and so forth. At the same time, a variety of devices is used to give certain groups a positive identity - to encourage the viewer to "imaginarily identify with the heroes" [emphasis original] (p.131), or negative identity - such as Somali warlords. US troops are described as being on humanitarian missions, providing relief to the reportedly starving population - while, as Machin and van Leeuwen (2005) suggest, their actual role in the conflict was probably damaging more than it was beneficial. Meanwhile, actual suffering is backgrounded, especially in the game.

The study thus shows a direct connection between institutionally-driven ideologies (of support for the US military) and ways in which they are realized and reinforced through visual discourse. I will return to aspects of Machin and van Leeuwen's findings in Chapter 3, as part of my methodology.

Van Leeuwen and Jaworski's (2002) article compares the photographs representing the Israeli-Palestinian conflict in the British Guardian newspaper, and the Polish Gazeta Wyborcza. Once again, it is shown how ideological stance influence representations of reality - the Guardian tends to show Palestinians more in victim or lone resister roles against Israeli troops, in a way romanticizing their identity; Gazeta Wyborcza shows the Palestinians primarily as terrorists, while at the same time representing the Israelis as a mostly abstract, distant force. This, again, shows the correlation between cultural and ideological differences, and patterns of depiction and allows van Leeuwen and Jaworski to draw very conclusions regarding the ideological positions of dominant political and social groups in Britain and Poland 
respectively - one of which sympathizes with the Palestinian cause, and the other does not.

Machin (2004) discusses image banks, companies such as Getty Images which collect and then sell stock photographs for use in the media. The study traces the rise of stock photography in news media, which took place simultaneously with the consolidation of these image banks, precipitated by computer technology which made storing, distributing and searching for images simple and efficient. Image banks allow journalists and editors of newspapers (both print and online) to search for stock photograph based on both simple terms and so-called 'conceptual terms' - such as 'freedom' and 'independence'. Stock photographs, as Machin notes, are generally 'decontextualized', making sure that "a photograph is more easily inserted into different contexts, and acquires a 'conceptual' feel” (p.320).

This has important ideological ramifications, since media articles about events and issues increasingly show 'conceptual', idealized representations instead of images originating from the real context of these events and issues. Most of these images are visually attractive - "posed, stylized, colour-enhanced" (p.335), showing models who represent generic types of people rather than individuals. Such images lack a definite sense of time and space, and can conveniently be re-used, unlike contextualized photographs. Perhaps even more concerning, from a critical point of view, are the types of images that image searches bring up. Machin (2004) observes that when one searches Getty Images for 'freedom', the results include several images of jumping, happy people, along with two images of US jet fighters, while 'capitalism' is not a possible search term and 'globalization' returns only "romantic images of global business" (p.333). Thus the types of stock photographs in image banks, their 
categorization, as well as their use are highly indicative of dominant ideologies involved in the production of the discourse.

Teo's (2004) study of national campaign posters examines three artifacts of a genre used by the Singaporean government to promote community values and majority interests. The three posters examined pertain to a campaign to increase national productivity, a campaign to encourage the use of Mandarin among the Chinese community, and a campaign to encourage public courtesy. Teo adopts a visual analysis framework based on an earlier (1996) edition of Kress and van Leeuwen's (2006) Reading Images, used extensively to inform the methodology in the present study. Ideational, Interpersonal and Textual aspects of the three images are examined and, importantly, paralleled to linguistic constructs through SFL - such as for example the reference to process types (p.196) in an earlier (1985) edition of Halliday and Mattheissen's (2004) An Introduction to Functional Grammar (as cited in the present study). I use a similar approach in Chapters 3 and 4, where applicable.

Teo (2004) systematically shows how Representational and Orientational patterns in the image produce images of harmony and positive relations, using a variety of devices - for instance showing a multi-ethnic selection of people with happy expressions from a close distance and in close proximity to each other (pp.195197) to symbolize public harmony; or bright, highly saturated colours and positive symbolic imagery such as a road leading toward a rainbow (pp.198-199) to suggest future prospects.

However as Teo (2004) suggests, the aesthetically-appealing imagery reveals not only positive aims of the government, but also underlying tensions between majority and minority groups within Singapore. For instance, the first poster (productivity campaign) carefully represents a wide, inclusive range of ethnic groups, 
but as Teo (2004) notes - "While ethnicity is carefully managed in the poster, other issues are more problematic. [...] The under-representation of females, for instance, could be seen as reflecting a fundamentally patriarchal society" (p.206). Likewise, features like the authoritarian stance of the government reflected by the low vertical angle used in the image, paralleled by the use of imperative in text, represent a fundamental social tension in the government's role (p.208). Teo (2004) also criticizes one of the posters (Mandarin campaign) as overly-contrived in terms of symbolism and as a result - incongruent (p.208).

Crucially, what Teo (2004) demonstrates is that the visual aspects of the posters are a meaning system which is capable of reflecting ideological positions without having to rely on accompanying text, instead adding its own concrete elements of meaning. The framework he uses for analysis is functional and accounts for aspects of all three primary metafunctions (ideational, interpersonal, textual), which parallel the functions performed by text.

These studies therefore illustrate ways in which relationships between dominant ideologies and visuals features can be uncovered through CDA/Social Semiotics approaches informed by both awareness of context and a functional analysis methodology for visuals. The approaches used by these studies, especially Machin (2004), inform the analysis methodology and will be referred to in the Methodology chapter below. What makes these examples particularly relevant is that all of them apply a functional framework for visual analysis which originates largely from the work of Kress and van Leeuwen (2006) and draws to a large extent on parallels to SFL. 


\subsection{Media Discourse in the U.S. War on Iraq - Chouliaraki (2005, 2006a)}

As already noted, a primary starting point for the present study is Chouliaraki's (2005, 2006a) research which examines BBC television's coverage of the initial stages of the 2003 U.S. war on Iraq, which focused on the 'Shock and Awe' bombing campaign against Baghdad. While Chouliaraki observes strategies similar to ones noted in the studies above, what is most interesting about her findings is the discussion of the way in which aesthetic values are used to displace suffering in the discourse, thereby distancing the viewer and preventing him/her from making ethical judgments or identifying with either sufferers or their persecutors.

In both of her published articles on this topic, Chouliaraki $(2005,2006 a)$ draws on the work of pragmatic sociologist Boltanski, who in his (1999) book Distant Suffering discusses the way suffering is socially constructed. He brings up the notion of an 'ideal public sphere', free from the influence of institutional ideologies, where individuals would be able to respond to others' suffering in ways which are ethical an appropriate:

where a local suffering can be conveyed without deformation in such a way that it is there for everyone to examine it, that is to say, for all those who, from the fact of their receptivity arising from a lack of prior commitment, are free to examine this suffering and find themselves sufficiently affected by it to become committed and take it up as their cause (p.31)

This, of course, is the ideal - and Chouliaraki (2005, 2006a) stresses that in the real public sphere, the communication technology of today is such that we can see events of war (i.e. suffering) more closely and immediately than ever before, and yet news portrayal actually distances this suffering significantly (2005, pp.144-145). The primary way in which this distancing is accomplished is through a pattern of 'aesthetization' or 'sublimation': "The sublime is a specific regime of pity that constitutes distant suffering less through emotions toward the sufferer and primarily 
through the aesthetic appreciation derived from the horror of the suffering itself." (2005, p.153) This is accomplished in the BBC coverage through imagery - primarily showing explosions in night-time Baghdad, accompanied by voice commentary describing the situation in sensational terms. The visuals employ a'detached' perspective, which "makes a distinct claim to impartiality as opposed to the 'involved' perspective" (2006a, p.266) Essentially, this aesthetic portrayal is made "without violating the principle of objectivity", and meant to be "contemplated at a distance and without human presence" (2005, p.156). Indeed, part of the distancing strategy is avoiding visual and verbal representation of both sufferers (Iraqi civilians) and persecutors (Coalition bombers) as animate, individual, concrete entities: the sufferer only enters the context of Coalition military movements "on the condition that her very humanity is cancelled" (2005, p.155). With it, the possibility of the viewer empathising with the victim is also cancelled.

Thus, Chouliaraki claims, 'aesthetic' portrayal creates a particular 'regime of pity' - an attitude suggested to the viewer, through which "suffering is construed as an object to watch and comment on, rather than to arouse emotion or a demand for action on the part of the spectator" $(2005, \mathrm{p} .151)$. This is achieved through active denial of individual, concrete identity to civilians, somewhat similarly to the way this is accomplished in Black Hawk Down, as analyzed by Machin and van Leeuwen (2005), but in an even more 'radical' fashion: "the clinical narration of suffering, the establishment of a radical distance from the location of suffering or the refusal to humanize the sufferer may indeed come to block an active relationship between spectator and sufferer" (Chouliaraki, 2006a, p.265). The portrayal uses virtually every possible technique to distance the viewer from suffering and more notably, the 
sufferer - and thus makes any efforts to criticize the events at best difficult: after all, it is difficult to criticize unseen violence against a highly diffused, impersonal entity. Referring once again to Boltanski's (1999) concept of the 'sublime' or 'aesthetic', Chouliaraki (2006a) discusses how this is accomplished without ever bringing up the idea of ethics or morals, keeping the viewer satisfied with their own (safe) position relative to the discourse:

The moralization of the spectator now takes place through a mechanism of 'sublimation', the representation of suffering through an aesthetic register that discourages spectators from feeling for or denouncing the suffering and invites them to contemplate the horror of the spectacle, the 'shock and awe' of the bombardment (p.276)

In other words, the denial of suffering through focus on aesthetic values - the 'Shock and Awe' produced by the scale of the bombings and their historical significance (as emphasized by the voiceover) - keeps the viewer from having to take sides: "Without overtly campaigning for the good nor even regulating the presence of good and bad on screen, the sublime plays upon absences" (p.279). This, of course, is deceptive: indeed, the viewer does take a side through passively approving the 'spectacle' with which he/she is presented.

What is significant about Chouliaraki's $(2005,2006 \mathrm{a})$ research is that, in my view, she captures what is a very complex, multi-faceted strategy that simultaneously involves alignment, distancing, abstraction and aesthetization - which carefully guides the viewer away from ethics and toward aesthetics. There are powerful ideological forces at work, employing simultaneously aspects of representation, organization and orientation. Unfortunately, the visual data used in the study is presented in, at best, generalized and vague terms. Thus, in the present study, it is a primary goal to examine in maximum detail a similarly complex, multi-faceted visual discourse of war, specifying features responsible for guiding the viewers toward 
ideological positions in terms of a detailed system of functional analysis of visuals. The interplay of perspective, identity, ethics and aesthetics will certainly be considered in the study, as already reflected in the research questions.

\subsection{Summary}

In the above chapter, I have specified the overall approach(es) taken within this study (Social Semiotics/Critical Discourse Analysis), discussed the origins of analytical tools for visual semiotics and their relationship to the tools of verbal analysis, and provided an overview of a number of research works which inform the theoretical approach, methodology, and research questions of the present study.

The following chapter will once again return to the sources reviewed here, in order to construct a detailed framework for subsequent data analysis. 


\section{Methodology and Data}

\subsection{Methodology - Introduction}

As already suggested, this study adopts a variant of Halliday's (2004) functional approach to language analysis that has been developed over time by researchers including O'Toole (1994), Lemke (1998, 2002, 2004, 2005), van Leeuwen (2005), and Kress and van Leeuwen (2006) to account for multimodal and visual discourse. In this chapter, I take a closer look at the functional view of visual discourse and construct an adjusted metafunctional framework to describe and analyze the data presented in the next chapter.

In constructing a methodology, the aims of the study should be considered foremost. For this study, the essential questions are, as stated previously in the introduction: 1) How are weapons, forces and actions of war presented? 2) How are images used within BBC (2003) and CNN (1998) documents on weapons and forces of the U.S. war on Iraq? 3) How is the viewer positioned relative to the war and the weapons in forces with which it is conducted?

These questions parallel Chouliaraki's $(2005,2006 a)$ work on the TV coverage of the U.S. war on Iraq and the aesthetization of suffering in general (2006b), as discussed earlier. Chouliaraki, looking at similar considerations (albeit through very different data) has focused on the following aspects in order to decode ways in which the portrayal of war achieves its ideological aims:

multimodality - the properties of language and image that construe the spectacle of suffering on screen space-time - the representation of proximity/distance of the viewer to the scene of suffering agency - the representation of action in the sufferer's misfortune

(Chouliaraki, 2006b) 
These three aspects form an essentially trifunctional approach - with 'multimodality', in the sense used here, being a narrowly-defined set of textual considerations; 'spacetime' looked at in terms of its role in positioning the viewer and thus forming the tenor of the discourse; and 'agency' being a basic aspect of ideational meaning (as per Halliday and Mattheissen, 2004; Kress and van Leeuwen, 2006). In her analysis, Chouliaraki $(2005,2006 a)$ has essentially taken a larger multimodal framework and narrowed it to features most relevant to the data set at hand. The present chapter will seek to do the same.

\subsection{Functional Analysis: Terminology}

Earlier in this paper, I outlined the functional approach to linguistic theory that has been developed largely from the work of Halliday during the 1970s (Halliday and Mattheissen 2004), and ways in which this theory has extended into visual analysis as well.

The model and terminology are relatively similar for both verbal and visual analysis, drawing primarily on Halliday and Mattheissen (2004) - starting with the level of Genre, as determined by the culture, and Register, as determined by specific situations within that culture (Eggins, 2004). Within any situation there are three constructs: Field, Tenor and Mode - the content of a situation, the types of interactions and relations established through the situation, and the nature of the communication itself (Young and Fitzgerald, 2006, pp.216-217). These constructs are expressed through three metafunctions or types of meaning: Ideational (Field), Interpersonal (Tenor) and Textual (Mode).

From this point on the methodologies of visual and verbal analysis certainly retain some similarity, but with different resources being analyzed, there is certainly some divergence in terminology. Below is the summary of some of the variations on 
the labels assigned to each of the metafunctions by theorists whose work informs this study:

\begin{tabular}{|c|c|c|c|}
\hline & Field & Tenor & Mode \\
\hline \multicolumn{4}{|l|}{ Language } \\
\hline $\begin{array}{l}\text { Halliday and } \\
\text { Mattheissen } \\
\text { (2004) }\end{array}$ & Ideational & Interpersonal & Textual \\
\hline \multicolumn{4}{|l|}{ Visual } \\
\hline $\begin{array}{l}\text { Kress and van } \\
\text { Leeuwen (2006) }\end{array}$ & Ideational & Interpersonal & Textual \\
\hline O'Toole (1994) & Representational & Modal & Compositional \\
\hline Lemke (1998) & Representational & Orientational & Organizational \\
\hline Knox (2007) & Representational & Interpersonal & Organizational \\
\hline
\end{tabular}

Are these terms interchangeable? They are to a point - although this is not to suggest the methodological approaches implied by them are identical. For this study specifically, Lemke's (1998) terminology will be adapted, though used interchangeably with the traditional Ideational/Interpersonal/Textual which is retained in the most recent and most comprehensive (from the methodological standpoint) resource, Kress and van Leeuwen's second edition of Reading Images (2006). The rationale for this is twofold. Firstly, this helps keep verbal and visual analysis parallel but separate, making connections between the two where they apply, but maintaining a distinction where the resources for expressing these types of meaning are simply too different between the two modes. Secondly, Lemke's (1998) terminology, in my view, better reflects what visuals - especially photographs - generally do within media discourse (and certainly within the analyzed data): they 'represent' (rather than create) what is already understood to be a reality, in ways which are much more direct than verbal resources; they 'orient' the viewers very directly in ways which go beyond interpersonal relations, and of which the notion of modality is only one (albeit very important) aspect; and they function to 'organize' discourse within and around them for which, again, I find 'composition' too narrow and 'textuality' too closely related 
to linguistic terms. It is useful to keep a distinction between linguistic and visual analysis within multimodal studies, and therefore I use Lemke's (1998) terminology where this distinction needs to be made clear in the present study.

As already noted, most of the current theoretical approaches to functional analysis of visuals draw on methods originally laid out by O'Toole (1994). O'Toole's approach certainly draws on visual art and art theory, and although a significant part of his categorizations apply to artworks specifically, much of it retains its relevance and, with adjustments, is made much more universally applicable than the title may first suggest. O'Toole adopts a metafunctional framework, and divides the realizations of each of the three types of meaning into four different units or levels of delicacy Work, Episode, Figure and Member - which, while certainly applicable outside of art as such, are certainly geared more towards accounting for complex conceptual structures which are not generally present in everyday media (such as the news discourse examined in this study). A textual component that is essentially grounded in art theory is the idea of the 'Gestalt' - a compositional 'wholeness' of sorts within an image, components of which are highly relevant to all imagery.

As a starting point for developing the analytical methodology for this study, O'Toole's (1994) model is summarized below: 


\begin{tabular}{|c|c|c|c|}
\hline Units |Functions & REPRESENTATIONAL & MODAL & COMPOSITIONAL \\
\hline WORK & $\begin{array}{l}\text { Narrative themes } \\
\text { Scenes } \\
\text { Portrayals } \\
\text { Interplays of episodes }\end{array}$ & $\begin{array}{l}\text { Rhythm } \\
\text { Modality } \\
\text { Gaze } \\
\text { Frame } \\
\text { Light } \\
\text { Perspective } \\
\end{array}$ & $\begin{array}{ll}\text { Gestalt: } & \text { Proportion } \\
\text { Framing } & \text { Geometry } \\
\text { Horizontals } & \text { Line } \\
\text { Verticals } & \text { Rhythm } \\
\text { Diagonals } & \text { Colour }\end{array}$ \\
\hline EPISODE & $\begin{array}{l}\text { Actions, events } \\
\text { Agents-patients-goals } \\
\text { Focal/side sequence } \\
\text { Interplay of actions }\end{array}$ & $\begin{array}{l}\text { Relative } \\
\text { Prominence } \\
\qquad \text { Scale } \\
\text { Centrality } \\
\text { Interplay of } \\
\text { Modalities }\end{array}$ & $\begin{array}{l}\text { Relative position in } \\
\text { work } \\
\text { Alignment of forms } \\
\text { Interplay of forms } \\
\text { Coherence of forms }\end{array}$ \\
\hline FIGURE & $\begin{array}{l}\text { Character Object } \\
\text { Act/Stance/Gesture } \\
\text { Clothing Component }\end{array}$ & $\begin{array}{lr}\text { Gaze } & \text { Contrast: } \\
& \text { Scale } \\
\text { Stance } & \text { Line } \\
\text { Characterization } & \\
& \text { Light } \\
& \text { Colour } \\
\end{array}$ & $\begin{array}{l}\text { Relative position in } \\
\text { episode } \\
\text { Parallelism/Opposition } \\
\text { Subframing }\end{array}$ \\
\hline MEMBER & $\begin{array}{l}\text { Part of body/object } \\
\text { Natural form }\end{array}$ & Stylization & $\begin{array}{l}\text { Cohesion } \\
\quad \text { Reference } \\
\text { (Parallel/Contrast/ } \\
\text { Rhythm) }\end{array}$ \\
\hline
\end{tabular}

Here one can see ways in which each type of meaning is realized at different levels of detail within visual discourse. Further in this chapter, aspects of this model and their incorporation into work of other theorists (Lemke, 1998; Kress and van Leeuwen, 2006) will be clarified and discussed. It is presented here in order to illustrate a functional, relatively inclusive set of analytical tools, which is what I aim to assemble in this chapter, before proceeding to analysis of the data.

Using Kress and van Leeuwen (2006) as a primary guide, and drawing on the theoretical work of O'Toole (1994), Lemke (1998, 2002, 2004, 2005), Kress and van Leeuwen (1992, 2001, 2002), van Leeuwen (2005) Jewitt and Oyama (2001), Iedema (2003) as well as applications of multimodal approaches by Chouliaraki $(2005,2006 \mathrm{a}$, 2006b), Knox (2007), Machin (2004), Machin and van Leeuwen (2005), van Leeuwen and Jaworski (2002) and others, I propose the following model for analysis in the present study: 


\subsection{1 - Adjusted Analytical Framework}

\section{Ideational/Representational}

- Narrative Structures

o Processes

- Transactional vs. Non-Transactional

- Vectors

- Participants

- Individual vs. Diffused

- Circumstances

- Conceptual Structures

- Classificational Structures (Taxonomies)

- Analytical Structures

- Topographic Structure

- Topological Structure

- Temporal Structure

o Symbolic Structures

\section{Interpersonal/Orientational}

- Perspective (Attitude)

- Size of Frame (Distance)

- Horizontal Angle

- Frontal vs. Oblique: Engagement

o Vertical Angle

- High vs. Low: Power

- Gaze: Offer/Demand

- Narrativization / Origin of Perspective

- Ordinary vs. Privileged Positions

- Modality

- Depth of Perspective

- Clarity/Detail - Participants, Background

- Colour - Differentiation, Modulation, Saturation

$\circ$ Lighting

- Coding Orientation

- Naturalistic

- Sensory

- Technological

\section{Textual/Organizational}

- Coherence

- Genre (generic type)

- Function within text

- Patterns of reference 
- Cohesion

- Framing

- Salience

o Linearity

o Information Value

- Left/Right (Given/New)

- Top/Bottom (Ideal/Real)

- Centre/Margins

This set of features informs the analysis presented in Chapter 4 . The rest of this chapter will proceed to explain, expand and clarify the features listed above, with special consideration for the ideological work they can be used to accomplish.

\subsection{Ideational / Representational Function}

As Kress and van Leeuwen (2006) suggest, resources used in conveying ideational or representational meanings must be "able to represent objects and their relations in a world outside the representational system." (p.42) Ideational resources, in other words, are a system of choices used to establish a field of discourse (Young and Fitzgerald, 2006, p.220) through which a reader/viewer may know what activity is taking place (i.e. being described or portrayed). Speaking strictly in terms of visual discourse, Knox (2007) states that "Representational meanings function to portray the experience, or a version of 'the way things are' (p.22, emphasis original).

\subsubsection{Narrative Structure}

One of the most primary element of representational meaning is accomplished through narrative structure, which includes the three most essential elements of any situation: processes, participants and circumstances. In visual discourse, participants are defined as distinct, salient, readily recognizable entities. They can perform active and passive roles (e.g. Actors and Goals). Processes are essentially relations, actions and reactions in which the participants are involved; these can be non-transactional 
and transactional, and are generally indicated by a system of vectors (linear, directional structures within the image) and geometrical symbolism. Circumstances are generally formed by less salient, secondary entities within images which are in non-vector-based relations with the primary participants. (Kress and van Leeuwen, 2006)

Within the system of narrative choices, one of the most powerful ways to present ideological positions lies simply in the inclusion or exclusion of participants, processes, circumstance or their aspects. In any type of imagery, it is virtually impossible to represent all aspects of a participant, process or circumstance. What is represented is termed by Kress and van Leeuwen (2006) as 'criterial aspects' of an object: "it is never the 'whole object', but only ever its criterial aspects which are represented" (p.7). The conception of criterial aspects in an image translates directly into the representational resources it employs.

The choice of what is to be represented needs to be made for any image - and can be made in a very strategic fashion, suggesting that the criterial aspects of an object or action presented in the image are a true and exhaustive representation of this object or action. This can be reinforced through patterns where the same selection of criterial aspects is made over multiple images. Within the data, for example, a pattern is observed where in Coalition vehicles, items like weapons and antennas always figure prominently (and are thus criterial in the context) but humans are entirely or almost entirely absent - contributing to the pattern of inanimate agency, which greatly assists in the framing of war as a technical process, thereby distancing the viewer from ethical questioning or sense or responsibility. 


\subsubsection{Processes}

The simplest way to explain processes is through a brief discussion of the same concept linguistic analysis, where processes are essentially what is realized by verbs actions, events and states. Processes necessarily include at least one participant (although certain structures can be used to conceal this participant); they can also include multiple participants involved in transactions. In systemic-functional linguistics, the following process types are distinguished: material (denoting action), mental (thoughts, emotions, senses), verbal (communication), behavioural (patterns of behaviour), and relational (existence, identity, possession) (Eggins, 2004).

In visual terms, most narrative processes are expressed through vectorparticipant relationships; in static imagery, geometric symbolism plays a particularly important role in denoting processes (Kress and van Leeuwen, 2006). Material, mental, verbal and behavioural processes expressed verbally translate relatively directly into visual meanings:

\begin{tabular}{|l|l|}
\hline Visual narrative processes & Linguistic narrative clauses \\
\hline Non-transactional action & $\begin{array}{l}\text { One-participant material process } \\
\text { ('action') }\end{array}$ \\
\hline Unidirectional transactional action & Two-participant material process \\
\hline Event & $\begin{array}{l}\text { Passive transactional clause with agent } \\
\text { deletion }\end{array}$ \\
\hline Bidirectional transactional action & - \\
\hline Non-transactional reaction & Behavioural process (field of looking) \\
\hline Transactional reaction & Mental process: perception (visual only) \\
\hline Mental process & Mental process (cognition and affection) \\
\hline Verbal process & Verbal process (quotation) \\
\hline- & Verbal process (affection) \\
\hline Conversion & - \\
\hline
\end{tabular}

(Kress and van Leeuwen, 2006, p.78)

Relational processes, which include Existential, Attributive, Identifying and

Possessive relations, are generally expressed visually through conceptual structures

(Kress and van Leeuwen, 2006, p.59), discussed further below. 
The choice of processes is a strategic one, and can influence the entire interpretation of discourse by altering the nature of the narrative being presented. For the purposes of this study, a particular focus will be on distinguishing transactional and non-transactional action processes, and reactions. Action processes can be represented in very concrete and transactional terms, or in abstract and nontransactional terms. For instance Chouliaraki $(2005,2006 \mathrm{~b})$ focuses significantly on tendencies to favour representing abstract, highly generalized, and technical processes over concrete, transactional material actions. In the present study, for instance, the difference between choosing an illustration of a soldier in the process of walking, a soldier in the process of shooting, a soldier smiling, or a soldier expressing anger to accompany an article about a military unit has a significant effect on the way the role of this unit will be interpreted by the reader/viewer.

\subsubsection{Vectors}

As noted above, narrative processes are visually realized through vectors. Kress and van Leeuwen (2006) define vectors as directional structures formed by strongly defined lines within an image: "In pictures, these vectors are formed by depicted elements that form an oblique line, often a quite strong, diagonal line [...] The vector may be formed by bodies or limbs or tools [...] but there are many other ways to turn represented elements into diagonal lines of action." (p.59) The strength of vectors and the clarity of their representation may vary; a strongly defined vector generally implies a dominant narrative or, in material terms, a powerful action. One of the ways in which vectors are commonly realized in abstract, diagrammatic imagery is through the use of arrows and triangular forms which explicitly produce diagonals and symbolically indicate direction. Vectors define narrative structures - they are something narrative structures have and conceptual structures do not. 
Vectors can realize transactional processes by pointing from one participant to another, or non-transactional processes by pointing outward from a single participant. It is possible, especially in abstract imagery, for vectors to be represented without clearly-defined participants, thus concealing or at least abstracting agency. The key aspect of vectors is their directionality: any vector must, as Kress and van Leeuwen (2006) suggest, indicate a direction: "features of directionality must always be present if the structure is to realize a narrative representation" (p.59). This directionality is easily realized in abstract imagery; in more naturalistic images, it frequently relies on subtle structuring of diagonal lines, or the orientation of participants and the viewer's ability to recognize them - for instance, any person in the modern Western society looking at an image of a car on a road should be able to recognize which direction the car is suggested to be moving in, simply through the car's external features.

Otherwise, if there are strongly-defined but directionless linear structures in an image, Kress and van Leeuwen (2006) suggest these to be realizations of analytical structures, showing relations between participants, rather than narrative vectors which indicate actions and events.

\subsubsection{Participants}

As already noted above, participants represented visually in an image are defined as distinct, salient entities (Kress and van Leeuwen, 2006, p.48) that are distinguished and connected by relative positioning, size, and vectors - in other words, involved in or connected through processes. They can take active roles (actors, sensers) - being the origin or subject of a process, or passive roles (goal, phenomenon) - being the receiver or object of a process. Under certain conditions, participants can be unrepresented - implied, positioned outside the frame or concealed, in a similar way that passives can be used in text to withhold overt agency (Eggins, 2006). 
The choice of represented participants is of paramount importance in any discourse. The choice to include or exclude a participant, or place a particular type of participant in an agent or goal role, is an ideologically powerful one. In discourse related to war, this is all the more important - since the reality of war is material violence and suffering. Chouliaraki $(2005,2006 a)$ notes that pity and responsibility for suffering can be assigned or, conversely, denied through choices of representative participants. In the TV imagery Chouliaraki observed on BBC, "The sufferer of the bombing is represented largely in non-human terms. [...] The sufferer is also verbalized as a diffused entity." (Chouliaraki, 2006a, p.272) This means that in representing the material processes of war (i.e. bombing, in this case), the entities representing the sufferer were non-human ('targets', 'positions') and often presented as diffused entities ('the city') - rather than as individual people. 'Unlike the references to 'anti-aircraft gunners' or 'emergency teams,' which are simultaneously visualized in a spectacular manner, the 'ordinary Iraqi' is a significant visual absence" (p.273). There are significant ideological implications to this: inanimate, distributed entities are not something that is capable of suffering, and are not something that the viewers/readers can directly identify with and sympathise - rather, only something they can observe. This is enhanced by the actors in the process of war being treated in the same way: "the diffusion of the persecutor [means that] representation of suffering in this piece of the footage systematically steers away from the emotional engagement with the figures of pity and proposes the spectator to watch a different approach to the element of suffering." (p.273) As noted earlier, this approach offers the viewer the possibility to observe the war as 'sublime' - an aesthetically- and historically-significant spectacle. Similar observations are also made by Machin and van Leeuwen (2005) in their study of war discourse in video games - where they 
suggest that rather than being presented as individuals, participants in violence are represented generically and 'collectivized'. They also emphasize the importance of naming and categorization of participants: only the actors towards which the player is intended to have a relation (positive or negative) are named, with others simply placed into categories such as 'enemies', 'civilians' and 'elite soldiers' - and visualized according to group characteristics rather than individual ones. This, again, places the player in a position to observe the discourse impersonally and focus on aesthetic elements and appearances, while remaining distant from any individual suffering.

Thus, in the present study the types of participants represented and ways in which they are identified - as animate or inanimate, individual or diffused - will be carefully observed.

\subsubsection{Circumstances}

According to Kress and van Leeuwen (2006), circumstances in visuals are non-vector relations and items in the discourse that do not appear to have a direct role in the process, but rather indicate peripheral meanings - Setting, Means and Accompaniment (p.72). Setting is frequently realized through the objects and attributes of the image's background; Means is essentially an instrumental relation whereby an action is accomplished by an actor with the help of an object (e.g. a soldier shooting by means of a gun); and Accompaniment is realized through visual collocation that places an object in the same context as a participant, but without specifying a role for it.

In linguistic analysis, Eggins (2005) also outlines circumstances of Manner, Extent (time), Cause, Matter and Role. Although most of these are usually expressed through conceptual structures in visuals, one could argue that it is at least possible for Manner to appear in much the same fashion as means or accompaniment - for 
example in the data, there are a number of images where one could argue that smoke trails from rockets or wakes from ships indicate the speed and power with which the action takes place (although these could be simply classed as vectors). Extent, Cause, Matter and Role are more symbolic types of meaning which are difficult to depict overtly through narrative representation, and in visuals are primarily expressed through conceptual structures, which are discussed further below.

In the present study, circumstances will be examined as far as possible. Presentation of setting, in particular, can be a strategic choice in regards to having the viewer identify with, or conversely - be distanced from, a scene presented in the image. A rich, recognizable background makes a scene more specific and identifiable; absence of background, conversely, abstracts the scene - along with the actions and participants it contains. In his study of stock photographs used in the press, Machin (2004) observes a consistent pattern of decontextualization - which implies abstraction of backgrounds, or even their outright removal. This has increasingly become a standard in photographs appearing in the press, which as Machin suggests increasingly favours re-usable, generic stock images over specific, contextualized photos: "by means of decontextualization a photograph is more easily inserted into different contexts, and acquires a 'conceptual' feel" (p.320) This 'conceptual feel' is in itself an abstraction and distancing strategy, since as Machin notes, "image bank images tend to lose their origin in time and space" (p.325), thus presenting the viewer with a perspective that is devoid of specificity, which is achieved through poorlyarticulated circumstances. As many of the publicity photographs discussed in the analysis originate in military and private image banks, this is a very relevant consideration in this study. 
In certain cases, choosing to represent an entity as a circumstance rather than a participant is also a significant ideological choice. As Machin and van Leeuwen (2005) observe in the Black Hawk Down video game and film, civilians are frequently backgrounded in the presentation (p.135), which does not remove them from the discourse, but by treating them as circumstances rather than participants the discourse minimizes the player's identification with their suffering: they are merely a part of the setting and to a large extent a cause, but they have no interactive role and thus become in effect less human.

Finally, circumstances in visuals can be an important way of specifying processes and participants. Machin (2004), in discussing the role of props in stock photography, notes that "Props are used to connote not only the setting but also the identities of the actors and the nature of activities, but in terms of 'types' rather than individual entities" (p.322) He suggests, for instance, that a model could be photographed with a laptop (as accompaniment) and would be understood to be a businesswoman; then, the same model could put on a hard hat and be understood as a construction worker. These collocations can be a powerful symbolic strategy to represent groups and types, and is certainly very important in a context where it may be ideologically advantageous to typify 'soldiers', 'civilians' and 'enemies' and thus diffuse identities of individuals that may be part of them.

Given the nature of the data, analysis in this study concentrates primarily on circumstances of setting and considers contextualization/decontextualization as one of the most important strategic choices.

\subsubsection{Conceptual Structures}

While narrative structures form the core of ideational meaning in visual discourse, there are aspects of representation which they do not capture. Consider, for instance, 
the earlier discussion of processes, where relations (as represented in language through relational processes) are generally presented in ways other than vectorparticipant relations. A more extended definition of representational meaning, provided by Lemke (1998), is as follows:

In visual depiction, this is the figural or representational function that presents to us a scene whose elements we can recognize and which have comprehensible relations to one another in terms of the typical scripts of that scene. This is what tells us what we are being shown, what is supposed to be "there", to be happening, or what relations are being constructed among the elements presented. (p.93)

The recognition of identities, 'comprehensible relations' and 'typical scripts' relies in large measure on conceptual structures within visuals.

Kress and van Leeuwen (2006) define three categories of conceptual structures in visuals: Classification structures (taxonomies), Analytical structures (part-whole relations), and Symbolic structures. Needless to say, conceptual structure is of particular importance to complex, technical imagery which needs to account for more than simply surface appearance of processes and participants - however elements of conceptual structure are present in naturalistic images as well. Below is a brief summary of the three types of conceptual structures.

\subsubsection{Classification Structures (Taxonomies)}

Classification structures refer primarily to taxonomical relationships within a context (Jewitt and Oyama, 2001). Taxonomies can be expressed overtly in diagrammatic imagery, by directly charting relations between objects, and also covertly elsewhere. Taxonomies can include multiple levels, with subordinate and super-ordinate members. Visual similarity in size, shape, and placement suggests the belonging of objects in the same class and on the same level; difference places objects into subordinate and super-ordinate roles. (Kress and van Leeuwen, 2006, pp.45-46) 
The simplest and most overt type of Classificational structure is a tree diagram, where elements are organized into classes directly and relations are determined by vertical and horizontal placement. However, only one such diagram is found in the entire data set for this study - and plays a very minor role, in explaining Iraqi command structure on the CNN website. The more important type of Classificational structure for this study, then, is covert taxonomy - which is produced through symmetric sets of participants within an image (Kress and van Leeuwen, 2006), used to establish classes. In the data, there are images which feature groups of participants such as soldiers or civilians, sharing similar features and having approximately equal prominence and position within the image - which helps establish them as classes. Recalling the pattern of 'typification' noted by Machin (2004) and discussed in the preceding section on Circumstances, this can be an important strategy in denying individual identity and diffusing/distributing agent and recipient roles to types and classes, thus denying the viewer a direct way of identifying with participants and providing a more abstract, aesthetic outlet for portrayals of suffering (Chouliaraki, $2005,2006 b)$.

\subsubsection{Analytical Structures}

According to Jewitt and Oyama's (2001) definition, Analytical structures "relate participants to each other in terms of a part-whole structure" (p.144). They are thus mostly concerned with attributive relations. As Kress and van Leeuwen (2006) indicate, these relations "involve two kinds of participants: one Carrier (the whole) and any number of Possessive Attributes (the parts)" (p.87). In the context of this study, examples of Carrier-Attribute relations in photographic imagery would be, for instance, illustrations of ships (carrier) with antennas, guns, structures on deck 
(attributes); or of soldiers (carrier) in uniform, with helmets and various equipment (attributes) and so forth.

Both structurally and in terms of purpose, Analytical structures are different from either Narrative structures or Classificational structures discussed above: "there is neither a vector (narrative process) nor compositional symmetry and/or a tree structure (classificational process). It serves to identify a Carrier and to allow viewers to scrutinize this Carrier's Possessive Attributes" (p.89). The latter point is particularly important: the focus of Analytical structures, as implied by the title, is to focus the viewer on analyzing the appearance and attributes of a subject. This is frequently further enhanced, as Kress and van Leeuwen (2006) suggest, by lowered modality - especially in the background - in order to allow focus on the Carrier. This echoes some of what has already been said regarding decontextualization (Machin, 2004) in terms of Circumstances - the focus on attributes is, essentially, an effect intended by decontextualization. This leads to precisely what Chouliaraki (2005, 2006a) calls 'aesthetization' - a focus on external attributes of the image's subject, which the viewer examines instead of considering the processes and implications of the discourse. As will be seen in the data analysis which follows, this is precisely what often occurs in presentations of weapons and forces.

Besides attribution, there are three specific types of Analytical structure that are particularly relevant to analysis of technical imagery (though can occur elsewhere): Topographic, Topological and Temporal structures.

Images where Topographic structure is employed are "read as accurately representing spatial relations and relative locations of the Possessive Attributes" (Kress and van Leeuwen, 2006: 98). One particular type of Topographic structure which is of relevance to this analysis is called an Unstructured Analytical Process, 
which presents "an unstructured map, accurate in scale, but without any visual indication of the possessive attributes relative to each other" (p.93). Kress and van Leeuwen (2006) go on to illustrate: "This can be seen, for instance, in advertisements which display all the parts that make up the engine of a car, or the full range of products manufactured by an advertiser, in order to impress the viewer with their sheer abundance" (pp.92-93). This is something that can be seen quite frequently in $\mathrm{BBC}$ imagery; and as the latter part of this example suggests, has a very concrete effect in terms of orienting the viewer - indeed, impressing him/her through attributes and quantity, rather than explanation of function. This once again recalls Chouliaraki's (2005, 2006a) 'aesthetization'.

By contrast to Topographic structure, Topological structure is intended to accurately represent relations between participants (Kress and van Leeuwen, 2006: 98). This is most obviously used in diagrams showing situations and functions of subjects. These do, in effect, create narratives - but in very abstract terms that deemphasize concrete, material dimensions (Kress and van Leeuwen (2006) even refer to these as 'quasi-narratives'). The use of such diagrams, as the data shows, can be a powerful tool to distance the viewer from suffering, by presenting the processes which produce it it in terms of relations in diagrams rather than more concrete terms.

Temporal structure refers to various ways in which static images indicate the flow of time, which is certainly relatively difficult to achieve without some form of symbolism. This can be done explicitly, through labeling time within an image - but can also be done implicitly, for instance by placing multiple depictions of the same participant along a line, with each introducing a gradual change in state (Kress and van Leeuwen, 2006). Within the data found in this study, use of temporal structure is certainly limited, and indeed as Chouliaraki (2005, 2006b) and Machin (2004) suggest, 
'timelessness' is a desired quality when abstract, distanced, relatively 'harmless' portrayals need to be produced.

Thus, Analytical structures deal with part-whole relations and are used when the viewer's attention needs to be shifted to attributes of a participant rather than any processes it is engaged in.

\subsubsection{Symbolic Structures}

Symbolic structure refers to the ways of indicating meanings about participants which are inherent to the participants and their attributes themselves (Kress and van Leeuwen, 2006). For instance, by being a member of the modern Western society, one can certainly recognize what traffic light colours mean: colour choices in this may seem arbitrary, but any driver would certainly be able to recognize the symbolism behind them. In their (2002) work on semiotics of colour, Kress and van Leeuwen mention it as something that, within an understood and established cultural system, can have definite symbolic meaning: "Starting with the ideational function, colour clearly can be used to denote specific people, places and things as well as classes of people, places and things, and more general ideas." (p.347) Likewise, in visual discourse related to military action, one can frequently recognize soldiers, civilians, and vehicles by external colours - which is something images in this data certainly do take advantage of.

One can also recall Machin (2004) and his discussion of the role of circumstances or attributes in creating instantly recognizable types - arguably, in large part through symbolic structure. Machin draws an interesting parallel between Medieval descriptions of saints and models in stock photographs: just as Biblical saints were recognized by items they carried or their dress in iconic images, so do modern Western people readily recognize types - be they businessmen, construction 
workers, soldiers or innocent civilians - by their appearance and items that accompany them. Their "individuality has been fully 'appropriated' [...] by the type they are to represent"' (p.323), Machin argues.

The importance of symbolism is to signal such types and identities to the viewer. It is particularly useful in considering some aspects of the appearance of military units (for instance, it is not difficult to identify a modern military plane simply by its shape), and distinguishing types of participants and, therefore, their suggested roles in the discourse.

\subsection{Interpersonal / Orientational Function}

The second function of any discourse is to convey interpersonal meanings, and thereby orient a viewer within the discourse. Knox (2007) offers the following definition: "Interpersonal meanings position the content of a text in relation to the producer and receiver, and in doing so construct a relationship between them." (p.22). A viewer is oriented relative to the discourse in two primary ways - firstly, through his/her implied positioning relative to participants and processes in an image (attitude/perspective), and secondly through ways in which the image articulates representations to appear true or 'real' to greater or lesser extents (modality) (van Leeuwen, 2005). Lemke $(2002,2004,2005)$ suggests that the antecedents of this type of meaning lie in personal and community-based values and attitudes: "These are the meanings by which we orient to each other in action and feeling, and to our community in terms of point of view, attitudes, and values" (p.305). Discussion of social and cultural antecedents for preferred attitudes (and thus orientations) will be included in analysis.

The rest of this part of the chapter will deal with two primary aspects of orientational meaning: perspective and modality. 


\subsubsection{Perspective (Attitude)}

Perspective is the way a viewer is literally positioned within an image. Within any type of static imagery which seeks to reproduce a naturalistic type of perspective (which is overwhelmingly common in Western culture, the viewer observes the scene from a fixed point, and through a fixed frame. The authors of any image select this perspective, and have a number of powerful ways of influencing the viewer's attitude at their disposal.

Any perspective consists of the following primary elements: size of frame (or distance), horizontal angle and vertical angle.

\subsubsection{Size of Frame (Distance)}

Size of frame refers to the relative size of a given participant to the total size of the frame (i.e. outer limits of the whole image), and can also be referred to as distance which, while technically incorrect (there is no such thing as distance in a twodimensional static image), accurately describes the effect of the size of frame on a viewer's perception. This distance, however, is not simply physical. Consider the following account of the implications of size of frame configurations:

Images can bring people, places and things close to the viewer or 'keep them at arm's length'. In everyday interaction the norms of social relations determine the distance we keep from each other. This translates into the 'size of frame' of shots. To see people close up is to see them in the way we would normally only see people with whom we are more or less intimately acquainted. Every detail of their face and their expression is visible. We are so close to them we could almost touch them. They reveal their individuality and their personality. To see people from a distance is to see them in the way we would normally only see strangers, people whose lives do not touch on ours. We see them in outline, impersonally, as types rather than as individuals. This does not mean of course that the people we see represented in close-up are actually close to us, or vice versa. It means they are represented as though they belong or should belong to 'our group', and that the viewer is thereby addressed as a certain kind of person. (Jewitt and Oyama, 2001, p.146) 
This effect is certainly most sharply apparent with human participants; however it extends to inanimate objects as well. For instance, as Kress and van Leeuwen (2006) state, a perspective which positions the viewer close to an object creates an impression - "as if he or she is using the machine, reading the book or the map, preparing or eating the food"' (p.125). A size of frame is considered to represent close distance when the subject of the image exceeds the size of the frame, with only parts of it visible within the image. This impression can be further enhanced by cutaways and representation of only specific parts of a subject rather than the whole. While socially the closest, this presentation can be confusing. Kress and van Leeuwen (2006) suggest that medium distance, where the subject is shown in full but without exceeding the size of frame, is often favoured in photographic imagery. In this way, features of the appearance of the object can still be shown in relatively high detail, while also showing its overall form. This is a distance that is generally preferred in advertising, and considered to represent a relatively privileged position (pp.125-126). At longer distances, where the subject only fills a small part of the frame, the social distance is much greater: "there is an invisible barrier between the viewer and the object. The object is there for our contemplation only, out of reach" (p.128) - in other words, it is out of the viewer's access, providing a view from "behind the gates" (p.128) only.

The difference which size of frame makes is one of social distance; generally speaking, the closer the perspective, the closer the relation suggested between the viewer and the reality represented in the image. 


\subsubsection{Horizontal Angle}

The horizontal angle in visual orientations relies on a simple geometric effect: the relationship between horizontal planes of the viewer and the scene inside the image. An image can present a scene from oblique or frontal angles - which, as Kress and van Leeuwen (2006) stress, "is not strictly an either/or distinction" (p.135) - an image can be predominantly frontal or predominantly oblique, but each of these includes a range of possible angles, the precise limits of which depend more on the viewer's subjective perception than on any specific geometric measurements. If an image is sufficiently detailed and presented in naturalistic perspective, parallel horizontal lines leading outward from the viewer (such as walls of buildings, for example) will converge at either one or two points if traced far enough. These convergence points can lie either within the frame or outside it. If there is a single convergence point, and it appears within the frame, the angle is considered to be frontal. If there are two convergence points that lie well outside the frame, the angle is considered oblique.

What is the implication of horizontal angle? Kress and van Leeuwen (2006) summarize this as follows:

The difference between the oblique and the frontal angle is the difference between detachment and involvement. The horizontal angle encodes whether the image-producer (and hence, willy-nilly, the viewer) is 'involved' with the represented participants or not. The frontal angle says, as it were, 'What you see here is part of our world, something we are involved with'. The oblique angle says, 'What you see here is not part of our world; it is their world, something we are not involved with.' (p.136)

The difference, thus, is one of involvement or engagement. With maximally frontal perspective, a viewer is drawn directly into a scene and into engagement (or, possibly, 'confrontation') with its participants - placed into the same 'world' as them. With maximally oblique perspective, the viewer is disengaged from the scene and takes on the role of observer - with participants being treated as 'phenomena' to be observed, 
rather than entities or person engaging the viewer (p.138). Between these, there is a range of options within the system, which Kress and van Leeuwen (2006) describe as "degrees of 'ourness' and 'theirness"' (p.139) which are regulated by horizontal angle. Finally, there is also an inverse variation of the frontal view - a back view, where the viewer's perspective puts him/her behind a participant. This can be used to suggest extreme disengagement, but also, as Kress and van Leeuwen (2006) suggest - trust, as exposing one's back to an observer can instinctively be considered a risk. A viewer placed behind a human participant is in a position of maximum safety - since in this case the participant can also be interpreted as a protector.

\subsubsection{Vertical Angle}

Vertical angle in visual representations, determined by how high or low a viewer's perspective is relative to the subject or scene of the image, mediates power relations. As Kress and van Leeuwen (2006) summarize, "if a represented participant is seen from a high angle, then the relation between the interactive participants (the producer of the image, and hence also the viewer) and the represented participants as one in which the interactive participant has power over the represented participant - the represented participant is seen from the point of view of power" (p.140) - and vice versa.

Low vertical angle (relative to the represented participant) can be used when a subject needs to be shown in a way that is impressive, powerful, or even threatening; high vertical angle suggests control, relative safety, and perhaps most importantly privilege in relation to the represented participant. The choice of vertical angle can be used to place the viewer into favourable or unfavourable positions relative to a subject, and thus help align or distance a viewer from it. This is a powerful method of 
orienting the viewer, and is all the more effective in combination with the other two aspects of perspective, which is detailed below.

\subsubsection{Perspective - Combination}

Researchers whose work has been mentioned so far in this study have found patterns of perspective to be very important in enforcing ideological positions in the discourse. It is standard practice in visual analysis to code the three primary aspects of perspective (Size of Frame, Vertical Angle, Horizontal Angle). Machin and van Leeuwen (2005), for instance, distinguish between features such as 'MLS' (medium long shot), CS (close shot); 'sideview', 'front view'; 'high', 'low' and so on. The way in which these features influence social distance, engagement and power is very explicit and important.

As Chouliaraki finds in her (2005) study of TV presentations of the U.S. war on Iraq on BBC, the viewer tends to get 'fixed' in a certain perspective, becoming static and showing one specific view of the war instead of moving through space and showing multiple perspectives on the war. In the case of BBC TV imagery in March 2003, Chouliaraki finds that the most predominant perspective is that seen from the hotel where reporters are positioned - a broad panorama of Baghdad taken from a relatively high position, with the strikes seen as flashes in the distance. In her (2006a) publication on the same topic, Chouliaraki calls this a 'detached perspective' - "the perspective of 'detached' observation makes a distinct claim to impartiality as opposed to the 'involved' perspective, and, therefore, it construes a distinct type of public space within which the spectator makes decisions about the suffering he or she witnesses" (p.266). She goes on to suggest that this combination of perspective features (long distance, oblique and high angle) has a powerful effect on identifying 
the viewer's place and restricting the interpretations of the discourse that the viewer could make:

Despite the total visibility that this view offers, or precisely because of this, spectators of the 'update' are simultaneously kept resolutely outside the scene of action. They are onlookers, watching the action from a safe distance. One consequence of the combination between distance and total visibility is 'detached' observation, a witness position that turns the reality of the war into a tableau vivant. (p.267)

Another way in which involvement relates to perspective, through distance as well as angle, is provided by Kress and van Leeuwen (2006), who state that at closer distance, the relations between viewer and participants/objects are "dialogic", while at longer distances they become "monologic" (p.129). It is thus reasonable to assume that if authors of an image seek to engage the viewer in observation (instead of direct involvement) and suggest interpretations rather than encourage discussion, they will choose 'detached', 'monologic' perspectives from longer distances and oblique, high angles. Patterns of these features will be noted carefully in subsequent analysis.

\subsubsection{Narrativization}

One basic question regarding perspective is: when perspective is present in an image, whose perspective does one see? In other words, what/whose position would a viewer need to be in to obtain the same view in the real world?

Kress and van Leeuwen (2006) use the term 'narrativization' to describe specific origins of perspective, stating that "in many cases there is no immediately apparent motivation for the point of view (and for size of frame)" (p.143). The viewer 'intrudes' into a picture, to be placed in a position of that or other participant within the context, giving him/her a virtual role in the discourse. "One can, and perhaps should, always ask, 'Who could see this scene in this way?', 'Where would one have to be to see this scene in this way, and what sort of person would one have 
to be to occupy that space?"' (p.143). This can be a powerful alignment strategy, as a viewer would naturally be aligned with the participant role from which the image is taken, and relations implied by that role.

Recalling Chouliaraki's $(2005,2006 a)$ notion of 'detached' perspective, it is also important to consider whose perspectives are denied in a given discourse. There are many different ways to witness the same event, with video technology as pervasive as it is, views from involved perspectives in a war are possible - but only a limited number of these are presented in war discourse. Machin and van Leeuwen (2005) likewise emphasize this theme in their study of Black Hawk Down, where the viewer is consistently positioned in perspectives which favour involvement with U.S. soldiers, but are detached from not only the 'enemy' combatants, but also the civilians.

A particularly unusual perspective can be used to simply draw attention to the subject and its attributes - for instance, aerial photography of ships from medium distance, which is used extensively in the data for this study by both $\mathrm{BBC}$ and $\mathrm{CNN}$, is something that can only be done with special equipment, special access and military cooperation, and no doubt at great cost and beyond the access of an ordinary observer. The resulting photographs are, however, aesthetically striking to an ordinary vieweras they place him/her in a privileged position that would otherwise be inaccessible.

\subsubsection{Perspective in Scientific Images}

On the surface, it would seem perspective is largely irrelevant in scientific or technical imagery - after all, even the most elaborate scientific drawings only partially articulate perspective depth. A lack of complex perspective, however, also has its effects. Kress and van Leeuwen (2006) note that most scientific images (technical drawings, diagrams, maps, charts) use either directly frontal or top-down 
angles - which are powerful and 'priviliged' because they "neutralize the distortions

that usually come with perspective, because they neutralize perspective itself" (p.144). This is accomplished in two different ways: "The frontal angle is the angle of "this is how it works', 'this is how you use it', 'this is how you do it'. The top-down angle, on the other hand, is the angle of maximum power. It is orientated towards 'theoretical', objective knowledge. It contemplates the world from a god-like point of view" (p.145). This claim to objectivity is a powerful one - and certainly is used productively outside of scientific discourse. This type of images increasingly pervades more 'everyday' discourses, including the media - which is certainly reflected in the data for this study.

Of course, perspective has also been gradually filtering into these popularized uses of technical imagery. This will be considered further below, in the discussion of Coding Orientations.

\subsubsection{Gaze}

One last aspect of perspective, which relates specifically to human (or at least animate) participants, is gaze. Gaze is mediated through eye contact and facing, and realizes the system of offer and demand. When human participants are involved, gaze can be used to indicate a relation between participants - and involve the viewer directly and in powerful ways. Combined with a low vertical angle (which indicates power) and frontal perspective (which indicates maximum engagement), direct eye contact can place a direct demand on the viewer (Kress and van Leeuwen, 2006)- in a way similar to imperative mood in linguistic analysis (Eggins, 2004). A particularly powerful, confrontational form of demand is a threat and can cause the viewer to take on a hostile attitude towards a represented participant. Combined with high angle it can instead indicate offer and create a submissive relationship between viewer and 
represented participants. Aversion of eye contact can disengage the viewer from the discourse and make it more difficult for him/her to identify with the represented participants. Averted gaze can also simply indicate the viewer's involvement in 'selfreflexive', mental processes (Kress and van Leeuwen, 2006, pp.120-121), a high degree of concentration. When seeking to distance the viewer from the discourse, it is advantageous to avoid direct gaze. When seeking to, conversely, align the viewer with a particular position (or set of participants) in the discourse, gaze can be used to great effect.

Thus, gaze and facing realize the system of demand, and contribute to engagement and power relations between viewer and represented participants when humans are prominently displayed in an image.

\subsubsection{Perspective and Privilege}

The notion of privilege has already been mentioned in relation to perspective in several instances - especially in reference to distance and vertical angle, and due to its centrality to some of the arguments made in the present study, I would like to clarify precisely what type of perspective are implied by this term.

A privileged perspective displays most of the following features:

- presents a view from an unusual but aesthetically-interesting point in space

- presented in high technical quality and with high articulation of modal resources

- made with active cooperation from the subject

- uses high angle to place the viewer in a safe, powerful position relative to subject

- uses partially frontal angle to indicate some measure of engagement between viewer and subject

- uses moderate distance to distance the viewer somewhat, but at the same time keep the clearest, most detailed and complete view of the subject possible

Privileged positions are a powerful way to align the viewer, who is much more likely to identify with subjects on which they have privileged perspectives. As shown later 
in the data, this particular distinction is one of the most primary ways in which an 'us and them' position is established in relation to Coalition and Iraqi forces.

\subsubsection{Modality}

O'Toole (1994) defines the entire system of Interpersonal/Orientation meaning as 'Modal', placing particular emphasis on this set of semiotic resources. In any framework, it is certainly of no lesser importance in orienting the viewer than the system of perspective.

The system of modality originates from linguistic analysis, where it is expressed through auxiliaries, adjectives and adverbs. Eggins (2004), based on Halliday (1978), distinguishes between modalization and modulation, the first of which expresses degrees of certainty and usuality (p.172), and the latter - degrees of obligation or inclination (p.179). For instance, consider the difference between degrees of inclination in "I might do it", "I probably will do it", "I will do it", "I will certainly do it", "I absolutely will do it". Modality can be both positive and negative, and range from unmarked to highly modalized or modulated. Highly articulated modality is marked - it draws attention to itself, and beyond a certain point can be over-articulated and regarded as excessive, untrustworthy hedging.

The same, essentially, is true of visual modality, although the resources through which it is realized are quite different, and include aspects of depth, detail, colour and lighting in an image, which will be considered further below. Visual modality can be high or low, with the distinction summarized as follows:

LOW MODALITY = ideal, usual, normal, abstract, typical, generalized HIGH MODALITY = real, unusual, abnormal, concrete, individual, specific (adapted from Kress and van Leeuwen, 2006, p.154)

As this suggests, the concepts of 'reality' or naturalism, and the question of norm, are of foremost importance in visual modality. Visual modality is always judged against 
naturalistic norms in a particular medium - which are determined by current norms of a medium, as Kress and van Leeuwen (2006) suggest - "visual modality rests on culturally and historically determined standards of what is real and what is not, and not on the objective correspondence of the visual image to a reality defined in some ways independently of it." (p.163) For instance in photography, the norms of contrast, colouring and sharpness which are considered naturalistic are determined solely by the median quality of photographs that are commonly seen in common situations in modern society. These norms would have been very different a hundred years ago, for instance. When these norms of the medium at a given time are exceeded, "an image becomes 'more than real' - an effect which can be achieved not only in art (and is often the favoured modality in Surrealism), but also by means of the special techniques, materials and equipment of studio photography" (p.163). Articulation of visual modality is thus a system of semiotic choices that can be used strategically and with increasingly sophisticated and at the same time accessible technology for producing and altering photographs at a high level of fidelity, notions of naturalism and the 'real' are being challenged. This is reflected on the pages of current media as Machin's (2004) study of stock, studio-produced, decontextualized photography and its increasing domination over 'real', context-specific photographs that were previously a dominant type of visuals seen in the press. It is still, however, possible to speak of naturalism in broad terms, as the ordinary, unaltered photograph is still a notable presence in media and elsewhere.

The set of eight modality markers proposed in Kress and van Leeuwen's Reading Images (2006) is summarized below: 
(1) Colour Saturation - a scale running from full colour saturation to absence of colour (black and white)

(2) Colour Differentiation - a scale running from maximally diversified range of colours to monochrome

(3) Colour Modulation - a scale running to a fully modulated range of colours with many shades to plain, unmodulated colour

(4) Contextualization - a scale running from the absence of background to the most fully articulated and detailed background

(5) Representation - a scale running from maximum abstraction to maximum representation of pictorial detail

(6) Depth - a scale running from absence of depth to maximally deep perspective

(7) Illumination - a scale running from the fullest representation of the play of light and shade to its absence

(8) Brightness - a scale running from a maximum number of different degrees of brightness to just two contrasting degrees

(Adapted from Kress and van Leeuwen, 2006, pp.160-163)

The system, in my view, is intuitive and inclusive, echoing much of what had been proposed by O'Toole (1994) and Lemke (1998). In order to simplify the organization of the data in the present study and address the aspects of images most relevant to media and the portrayals of war, I propose to unite some of the modalities under 4 more compact headings:

1) Depth

2) Detail and Sharpness (Contextualization, Representation)

3) Colour (Saturation, Differentiation, Modulation)

4) Lighting (Illumination, Brightness)

In the analysis of photographs and diagrams in documents by $\mathrm{BBC}$ and $\mathrm{CNN}$, distinctions between saturation and modulation, for instance, are not as significant as the overarching choice of allowing one or more aspects of colour modality to exceed naturalistic norms for a given image. All of the dimensions of colour, when highly 
articulated, serve to draw attention and seek to highlight aesthetic (or sensory) dimensions of the image's subject. The same is true of other modalities.

\subsubsection{Depth}

Although images are flat, they can create a sense of depth through a variety of means including overlap of objects, use of focus, diagonals and convergence of lines in the image. Lighting, contrast and colour can also contribute to perception of depth. Naturalistic images generally have some measure of perspective, although this can vary to a great extent - ranging from frontal images of a flat surface to highly articulated vertical and horizontal angle, unusually narrow or wide lenses, strong convergence of vertical lines, and so forth (Kress and van Leeuwen, 2006, p.162). Non-naturalistic images can include varying degrees of depth through the arrangement of lines and shapes in an image; a very common partial use of depth is isometric perspective, which creates a sense of depth through alternation of clearlydefined horizontals, verticals and diagonals .

Articulation of depth is an important aspect in creating privileged perspective - as hyper-articulated depth gives the viewer not only a sense of the subject's twodimensional form and outline, but also produces a strong sense of three-dimensional form, something which requires managing perspective very carefully.

\subsubsection{Detail}

As Kress and van Leeuwen (2006) argue, the more detail is visible, the more specific and 'real' an image and its subjects are considered - although beyond a certain point, as any marking of modality, it becomes 'hyper-real' (a technique used thoroughly in Dali's surrealist paintings, for instance). Conversely, the lower the detail in the image, the more idealized or typical the subjects become. 
Recalling Machin's (2004) discussion of 'decontextualization' as a way of distancing the viewer, one can certainly apply the notion here as well. Absence of setting lowers modality: "shown in a void, represented participants become generic, a 'typical example' rather than particular, and connected with a particular location and a specific moment in time." (Kress and van Leeuwen, 2006, p.161)

\subsubsection{Colour}

As noted earlier in the discussion of symbolism, colour is not significant by itself but can be used productively as a marker within a context (Kress and van Leeuwen, 2002). Colour can be articulated along three different scales - saturation, differentiation and modulation. Kress and van Leeuwen (2006) suggest that the naturalistic standard for modern photographic imagery lies approximately $3 / 4$ of the way between lowest and highest possible articulation of colour (p.160), which assumes a reasonable but subdued range of colour. Above this level, colours become more sharply characterized - which, while less naturalistic, can be aesthetically attractive and more symbolically significant (provided there is an established contextual interpretation for the use of these colours).

\subsubsection{Lighting}

Lighting, brightness and contrast can be used to make an image appeal relatively ordinary if used within naturalistic terms, or suggest dramatic quality (something which is used to great effect in Baroque paintings, for instance). As already noted, lighting can interact with perspective depth in presenting the three-dimensional form of an object through the interplay of light and shadows (Kress and van Leeuwen, 2006). In ordinary, naturalistic imagery, lighting is usually incidental to the scene displayed; in studio photography and publicity photography in general, lighting is 
very much a science and used deliberately for emphasizing appearance, form and attributes of a subject - thus focusing the viewer on its aesthetic dimensions.

\subsubsection{Coding Orientation (Modality Configurations)}

As noted already, the notion of 'real' in any type of imagery is determined by the standards of the given medium. The term Kress and van Leeuwen (2006) use to summarize the divergent notions of the 'real' in visuals is Coding Orientations, distinguishing four primary types - Naturalistic, Technological, Sensory and Abstract (pp.165-166).

For instance, despite being physically flat, unable to emulate the dynamic response of human eyes to varying levels of light, and only able to represent a small fraction of the actual range of visible colours, photographs are accepted as 'real' in modern Western culture (Kress and van Leeuwen, 2006, p.165; Jewitt and Oyama, 2001 , p.151). Still, within those limits, it is possible to achieve a socially-acceptable representation of reality, of things 'as they are'. Jewitt and Oyama (2001) provide the following definition: "Naturalistic modality (modality = 'reality value') defines visual reality as follows: the greater the congruence between what you see of an object in an image and what you can see of it in reality with the naked eye, in a specific situation and from a specific angle, the higher the modality of that image" (p.151) This is the first, but not only type of reality that can be represented through imagery.

The second type of reality that can be represented through images is one that focuses not on appearance but functional accuracy: "graphs and diagrams can also be thought of as true to reality, as images that depict the world as it is, objectively, scientifically - and yet they lack all the characteristics that contribute to the photographic impression of reality" (Jewitt and Oyama, 2001, p.151) The notion of 'real' in scientific imagery is thus completely different from photographic imagery, as 
Machin (2004) indicates: "a photograph may be seen as showing only the surface, while diagrams can get underneath that surface by representing the structures common to a range of superficially different phenomena" (p.327). In this context, an image is only as real as its presentation of facts is complete, often at the expense of modality marking - "There is often no background, detail is simplified or left out, colour and depth regarded as superfluous" (Jewitt and Oyama, 2001, p.151). In other words, a scientific image regards any modality marking as important only if it contributes to technical accuracy.

The notion of 'real' in the third type of modality is in many ways the inverse of scientific 'reality', focusing not on factual accuracy of subjects but rather representing an aesthetic sense of them. In today's Western world, and especially in the media, these sensory images widely employ photographic technology - but in a way different from naturalistic representations:

Here what matters is neither the truth of verisimilitude, nor the abstract, 'essential' truth, but emotive, 'sensory' truth. From the point of view of photographic realism, such 'sensory' images will be less realistic, but this time not because of a reduction, but because of an increase in the use of the means of visual representation: uncannily fine detail, richer colour, a deeper perspective. (Machin, 2004, p.327)

Since this type of imagery engages the viewer directly through affective and aesthetic connotations of its visual richness, this approach to modality is favoured when the purpose of the image is to attract the viewer's attention and promote the subject of the image- for instance in advertisement, but also increasingly in the media. Machin (2004) describes stock photography as generally "posed, stylized, colour-enhanced" (p.335), easy for the viewer to identify - but only in vague, surface terms. For the use of this type of imagery to illustrate news stories and events in the media, Machin certainly spares no criticism - suggesting that sensory-oriented stock imagery's sense of reality is increasingly one of "a world organized into consumer categories and easy 
clichés" (p.334). As the findings of the present study show, the simplified, aesthetic reality of sensory imagery is indeed widely used to displace the other types of reality - in particular, a material reality of suffering in the context of war.

The fourth type of Coding Orientation, as Kress and van Leeuwen (2006) suggest, presents an abstract version of 'real', which is a low-modality configuration which uses highly simplified visuals to represent complex concepts, something that would be only interpretable by highly educated elite. For the purposes of this study, this orientation is not considered - simply because it is at odds with the functions of public media, which is directed at the wider population rather than the elites.

Kress and van Leeuwen provide the following definition of the concept of Coding Orientations, emphasizing their socially-motivated nature: "Coding orientations are sets of abstract principles which inform the way in which texts are coded by specific social groups, or within specific institutional contexts" (p.165)

The four Coding Orientations are thus summarized as follows:

(1) Naturalistic coding orientation (low modality) - seems to mean approximately the same for everyone, represents reality 'as is' - e.g. ordinary photography

(2) Technological coding orientation (low modality) - where scientific or technological purpose of the image is paramount - e.g. technical drawings, diagrams, blueprints

(3) Sensory coding orientations (high modality) - where colour and detail is a source of pleasure and affective meanings - e.g. magazine photos, advertisements

(4) Abstract coding orientations (low modality) - where complex realities and concepts are reduced to basic representations, which can only be interpreted by an educated elite - e.g. high art, academic contexts

(adapted from Kress and van Leeuwen, 2006, pp.165-166)

Within the data analyzed in the present study, the three relevant coding orientations (Naturalistic, Technological, Sensory) are used extremely productively to orient the 
viewer and distance him/her from the reality behind the discourse. The interplay between different concepts of 'real' is deliberate and ideologically-driven.

It is important to note that, as Kress and van Leeuwen (2006) observe, under certain circumstances and for special purposes, it is possible to compromise between different coding orientations. For instance, technological images can be partially 'naturalized' when drawn from perspective, and thus be made more naturalistic and acceptable, drawing on both notions of 'real' simultaneously (p.172). This is certainly something which is observed in this study in regard to diagrams produced by the BBC.

At the same time, Kress and van Leeuwen (2006) observe that in modern visual media, there is an increasing tendency toward coded - diagrammatic or posed imagery, rather than simply naturalistic presentations (p.29). Even naturalistic imagery is increasingly coded (p.30) - frequently representing conceptual and aesthetic notions of 'real' along with its usual function. This parallels the observations made by Machin (2004), who suggests that decontextualized, studio-produced sensory images are increasingly dominating news discourse where naturalistic, concrete, highly contextualized images were favoured. This tendency is noted in this study as well.

\subsection{Textual / Organizational Function}

The third function of discourse is to manage and organize the flow of information. Knox (2007) defines this function as follows: "Organizational meanings form interpersonal and representational meanings into whole texts which are cohesive and coherent" (p.22). It should be noted that the concept of 'text' does not indicate written linguistic artifacts, but rather refers to any type of semiotic construct. The notions of cohesion and coherence essentially refer to the internal organization of the text and to connections it has to other texts in the same context respectively (Halliday and 
Mattheissen, 2004). Lemke (2002) describes the way in which organizational meanings operate as follows: "Organizational meanings are largely instrumental and backgrounded; they enable the other two kinds of meaning to achieve greater degrees of complexity and precision. Most fundamentally, organizational resources for meaning enable us to make and tell which other signs go together into larger units." (p.305)

A more extended definition of this function of text by Lemke (1998), referring to the work of O'Toole (1994), notes some of the ways in which coherence and cohesion are realized:

Depiction deploys compositional (O'Toole, 1994) resources to organize the visual text into elements and regions, and to link disjoint regions by such features as color and texture. As material objects, depictions participate in interactions that define parts and unite them into wholes in the ecosystem networks where objects are viewed and used. (p.94)

Within this study, cohesion is discussed briefly in terms of the generic types of images, identified through surface features, as well as the relationships between images and their wider context - which in this case is mostly viewed through the relation between images and the articles they accompany, as well as through patterns emerging among images.

Coherence is considered in terms of how these 'elements and regions' are linked within images. This is accomplished primarily through the constructs of framing, salience, linearity and informational structure.

\subsubsection{Text-Image Relationship}

Of the literature reviewed for this study, Lemke's (2002) and Knox's (2007) work deal most directly with website layouts. While layout in general is outside the scope of this study, where I am primarily concerned with the images themselves, it is important to consider the layout in terms of the relationship between text and images, 
since the illustrations used by $\mathrm{BBC}$ and $\mathrm{CNN}$ work directly in conjunction with articles in which they are presented, and their meaning depends on their relation with the text to a significant extent.

Traditionally, images in public news media have served as illustrations essentially, in roles secondary to text. Their prominence and role, as Kress and van Leeuwen (2006) suggest, is changing - images are moving into more primary, salient roles and positions within text.

The relationship between text and visual for most of the data observed is a complimentary one, with images and text supporting frequently different meanings they make. This is reinforced through informational structure, which will be discussed further below.

Chouliaraki (2006a) discusses the relationship between language and images in television as one between a passive and an active element - with the images simply presenting reality as it is ("a 'look-at-this' mode of address") (p.267), while the language (verbal narration in the case of her study) explains the significance of this image ("a 'this-is-what-it-means' mode of address"). Despite this perceived passiveness, however, the images are not at all neutral - as Chouliaraki herself effectively shows.

Recalling once again the work of Machin (2004) on stock photography, another important development in the role of visual images should be noted - as more and more generic, decontextualized imagery is used in media, the role of images is changing due as they lose sense of time and context: "There has been a shift $[\ldots]$ from emphasis on photography as witness to photography as a symbolic system" (p.317). The result of this is that instead of showing a 'real' representation of a subject in the 
material sense, the images now play the role of representing a generalized, idealized essence of the subject - in many ways setting thematic structures for the entire text.

Finally, with the increasing shift to coded technical imagery in the media (Kress and van Leeuwen, 2006), visuals often take a dominant role, serving as not only illustrations but indeed complete, self-contained explanations that only rely on text insofar as labeling items requires.

These factors regarding the role of imagery are thus taken into account in the analysis of data. The types and functions of images relevant to the data in this study will be presented in further detail in the next chapter.

\subsubsection{Framing, Salience, Linearity}

In terms of coherence, the internal structure of images is governed by "three interrelated systems" (Kress and van Leeuwen, 2006, p.177) - Information value, Salience and Framing. These, along with linear structure of images, are discussed as part of the present section.

\subsubsection{Framing}

Framing refers to a presence or absence of framing devices that connect elements of an image - suggesting what belongs together, what belongs separately, and what belongs in a certain given context (Kress and van Leeuwen, 2006, p.177). Jewitt and Oyama (2001) offer the following definition: "The term 'framing indicates that elements of a composition can either be given separate identities, or represented as belonging together. In other words, framing 'connects' or 'disconnects' elements" (p.149).

Framing can be realized in a variety of ways. The most obvious is overt framing of scenes with borders - which is certainly common in the media. In this case, 
frames are usually formed by clearly-defined lines. Framing can also be implied by the geometric layout of an image - the patterns of horizontals and verticals dividing it into areas. Other means of framing include context - the background against which elements are seen; colour patterns; and as part of temporal structures (discussed above) - also rhythm, as Kress and van Leeuwen (2006) argue: “. From time to time the ongoing equal-time cycles of rhythm are interrupted by a pause $[\ldots]$ and these junctures mark off distinct units, disconnect stretches of speech or music or movement from each other to a greater or lesser degree." (p.203) These interruptions of rhythm can be used to separate elements (participants, processes) in an image, and to suggest that they do not 'belong together'.

Conversely, when there is no framing present, the suggestion is that elements shown are part of the same context, group or classification: "The absence of framing stresses group identity." (p.203) When multiple elements are present within a single, clearly defined frame, it is suggested that they naturally 'belong together' (pp.203204).

Framing can greatly assist in the production of some of the same meanings through Classificational and Analytical structure, and enhance either the production of distinct identities, or, conversely, assist with their 'diffusion' (Chouliaraki, 2005, 2006a). Organizationally, framing is instrumental in producing perspective, where distance and angle are mediated through size and type of frame. Overall, the role of framing in joining or separating elements and creating (or denying) identity is of prime importance in a visual discourse.

\subsubsection{Salience}

Salience refers to ways in which elements within an image attract a viewer's attention. It is used, in other words, so that "some elements can be made more eye-catching than 
others. This again can be made in many different ways, through size, through colour contrasts (red is always a very salient colour), tonal contrast - in short through anything that can make a given element stand out from its surroundings." (Jewitt and Oyama, 2001, p.150) The most salient element or elements in images become the subject and centre of the discourse, indicating to the viewer what an image is about.

As the definition by Jewitt and Oyama (2001) above suggest, a variety of methods including colour differentiation, contrast and size are used to indicate salience. There is no objective method of determining salience - it is always relative within an image, as Kress and van Leeuwen (2006) stress: "The viewers of spatial compositions are intuitively able to judge the 'weight' of the various elements of a composition, and the greater the weight of an element, the greater its salience." (p.202) Thus a blurry, low-saturation shape will be highly salient when presented in a decontextualized fashion, whereas a fairly well-defined object or even a person can hold very little weight in an image that presents a busy street scene with many other objects of greater salience in it.

Salience can interact with informational structure (discussed further below) and, for instance, indicate the importance of a Given against a New, or vice versa: "Regardless of where they are placed, salience can create a hierarchy of importance among the elements, selecting some as more important, more worthy of attention than others" (Kress and van Leeuwen, 2006, p.201). The hierarchical nature of salience mentioned here is also of note, and contributes to organization of Classificational structures (taxonomies).

Thus, salience is a means of organizing visual discourse by directly indicating which items are more important than others, thus indicating which elements the discourse is really about. 


\subsubsection{Linearity}

Linear structure refers to ways in which a viewer's attention may be directed from element to element in an image. In naturalistic images, linearity is generally incidental; but elsewhere it is possible for authors of the discourse to manage the way a viewer observes an image.

As Kress and van Leeuwen (2006) suggest, traditional printed text structures are always linear by necessity: "In densely printed pages of text, reading is linear and strictly coded. Such texts must be read the way they are designed to be read - from left to right and from top to bottom, line by line." (p.204) One of the most important features of Hypertext in online environment is that by contrast, it is highly non-linear - something that is of particular importance to Lemke's (2002) study of Hypermodal discourse. He notes that in non-linear structures - including images - the viewer's gaze wanders, but can be fixed where necessary by use of salience and vectors (p.301). This can be used to create reading paths, something that is particularly relevant in diagrammatic imagery for instance: "reading paths begin with the most salient element, and from there move to the next most salient element, and so on" (Kress and van Leeuwen, 2006, p.204).

By explicitly guiding the viewer through an image, careful management of reading paths can be used to suggest interpretations and point out points of importance within an image while (along with salience, modality, and other means) helping to sideline elements considered non-essential. This can certainly be an ideological choice in distancing and alignment of the viewer. Linear structures, therefore, should be considered as another means of suggesting particular meanings, and are analyzed where applicable in this study. 


\subsubsection{Informational value}

The concept of Information structure takes advantage of a system whereby the relative placement of elements in a semiotic space endows them with certain information value. Kress and van Leeuwen (2006) distinguish between three spatial relations, whereby elements within an image are endowed with depending on in which 'zone' they are in the image - left/right, top/bottom, centre/margin.

Left-right relations between elements of an image account for Given-New structures. If two elements are thus placed in a horizontal relationship, the element on the right becomes a Given - representing information that is implied to be known, familiar, standard or pre-supposed; and the element on the left becomes a New, which represents a new message or unknown information about the Given (Kress and van Leeuwen, 2006, p.180). This can be used in a variety of ways - for instance in website layouts with multiple columns and spaces (Lemke, 2002, 2004, 2005) where information be strategically placed in order to be represented as either Given (and thus known and proven) or New (and thus more interesting or unusual). When there are chains of multiple elements placed along a horizontal lines, one element can be simultaneously New (in relation to the element on its left) and Given (to the elements on its right), thus creating chains of 'Given News' (Kress and van Leeuwen, 2006, p.185). Since Given-New structures are theorized to be connected closely to written script (which in most Western cultures is left to right), Kress and van Leeuwen (2006) note that in other cultures which use languages such as Arabic, the direction of this relationship is reversed (since the script is right to left) (p.181).

Top-bottom relations realize Ideal-Real structures. Kress and van Leeuwen (2006) suggest that "For something to be ideal means that it is presented as the idealized or generalized essence of the information, hence also its, ostensibly, most 
salient part. The Real is then opposed to this in that it presents more specific information (e.g. details), more 'down-to-earth' information (e.g. photographs as documentary evidence, or maps and charts), or more practical information (e.g. practical consequences, directions for action)." (pp.186-187) Interestingly, the data in this study shows a pattern of relationship between 'details' (i.e. text) and photographs - with many images in fact used at the head of articles, which suggest that images, rather than the text, are preferred in representing the ideal. Recalling some of the patterns of decontextualization and the changing role of photographs in the press described by Machin (2004), this certainly makes sense.

Finally, Centre-Margin relations are responsible for creating subordinate relations. If an element is in the center, it is presented as the 'nucleus' of the discourse, with other elements placed in the periphery being subordinate to it (Kress and van Leeuwen, 2006: 196). As Kress and van Leeuwen suggest, Centre-Margin layouts are relatively uncommon in modern Western culture, although are still used on occasion. For the data in this study, this is not a relation that is used in a particularly productive way - but it is still useful to keep it in mind.

One important aspect of the three types of Information structures is that they can combine with each other. The diagram below illustrates the way in which GivenNew, Ideal-Real and Centre-Margin relations interact on a visual plane: 


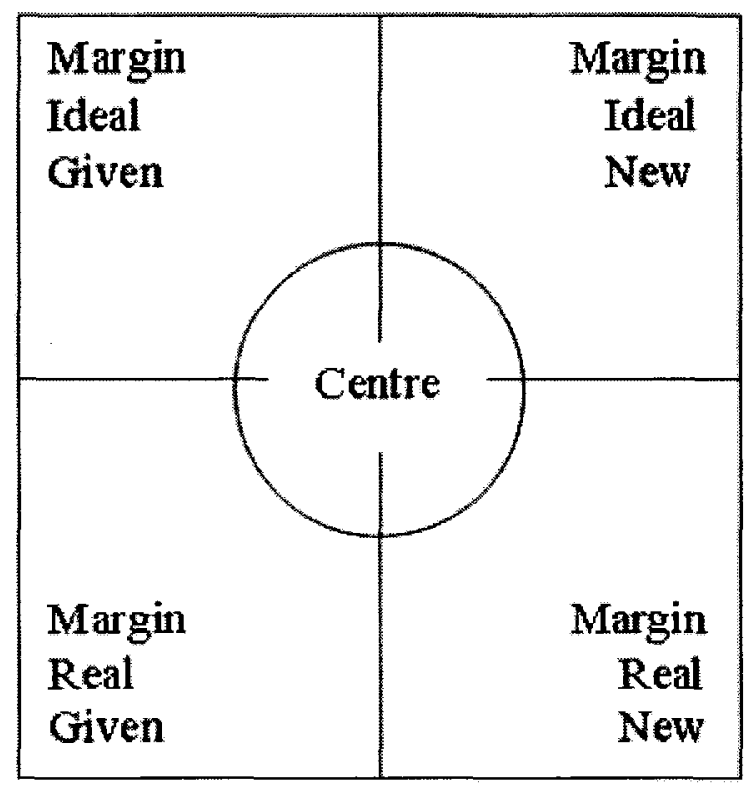

(from Kress and van Leeuwen, 2006, p.197)

It is through this interaction that the Centre field of an image attains another

role - that of mediator between Given and New, and likewise between Ideal and Real.

Within the present study, the structures of Informational value will be considered both within images and in explaining relations between image and text especially the latter, since there are notable patterns of both left-right and top-bottom dynamics in terms of placement of images and text that certainly help explain the function and features of these images. This will be further discussed in the Analysis chapter.

\subsubsection{Recurrent Patterns}

As already noted above, rhythm and patterning are an important means of organizing discourse. In linear discourse, it is possible to embed certain ideological orientations in stages and expect the viewer to gradually absorb their effect as he/she proceeds through the text (or visuals). However, online media is non-linear, as Lemke's (2002) work on Hypermodality suggests. Knox (2007), in his study of online newspapers, likewise stresses that it is impossible to predict a viewer/reader's path through online 
discourse. Thus, he states the following in regard to the way ideological orientations

are built into non-linear discourse:

As news stories become more atomized and the reading of news texts becomes more 'traversal', the ideological representations in a single verbal news text take on less and less significance, and the mosaic in which they are visualverbally positioned becomes increasingly important. A reader's interaction with an online newspaper is chaotic - a particular reading path cannot be predicted, and the path and exit point cannot be determined by the entry point and entry conditions. Over the course of time, however, collective order emerges from the chaos of individual trajectories, and patterns of meaning emerge. It matters less and less what a particular news text says (and it is increasingly likely to say very little in any case), and more and more what patterns emerge across the superordinate text. (p.48)

While Knox focuses particularly on what he calls 'newsbites' - a highly abbreviated text genre that is emerging in online newspapers, this observation is also very applicable to visuals. As the content of online media becomes increasingly visual, the unpredictable reading path of the viewer means that like nowhere else, emergence of superordinate themes and patterns across images is the single most important way of reinforcing ideological positions. The features of such images, in many ways, are positioned to be important only insofar as they support (or deviate from) these superordinate patterns. It is precisely for that reason that the present study is aimed foremost at uncovering these patterns. The portrayals of weapons on BBC and CNN obtain their powerful ideological effects in terms of aligning and distancing viewers through this patterning more than through any other means available in the discourse. 


\subsection{Data}

Having outlined the analysis framework for the present study, I now proceed to a brief description of the data, starting with the historical and technological context in which it is found. The remainder of this chapter will discuss the history and ideologies behind the representations of modern war in the media, briefly outline the development of 'new media' in online environments, and specify the selection and organization of the data used for the study.

\subsubsection{Modern War and the Media}

Since the present study examines two artefacts which originate from a rich and complex war discourse, it is certainly worth to step back and consider this wider context, which this section aims to do. The history of war and the media is a complex issue, so the present section provides only a brief and limited overview to inform the analysis, referring primarily to work by of Allan and Zelizer (2004) and Andersen (2006) on media and the war specifically, as well as Lewis (2007) on the culture of war which has developed in the United States over the past fifty years, both within the public and the military establishment.

As Andersen (2006) points out, for the majority of the Western public today war is not an immediate reality - it is, rather, something that is mediated and negotiated through depictions:

War is understood and interpreted, justified and judged through the images and narratives that tell the stories of war. Most civilians experience military conflict through the signs and symbols of its depiction, their impressions derived not from the battles in distant lands but from the manner they are rendered at home.

(xvi)

The development of communication technologies, especially the television and later also the Internet, has both accelerated and complicated ways in which this mediation takes place (Andersen, 2006: xxi). The media and the institutions of power have had 
to adapt to the capabilities of this new technology, which has allowed the public to access images and narratives of war in ways which are much more immediate and often more telling of the human suffering which accompanies war. As Adrian Lewis (2007) suggests in his book The American Culture of War, the Western, particularly American public have difficulty reconciling enlightened democratic ideals with violence and suffering - and for this reason technology has increasingly come to the forefront in the conduct of war (Lewis, 2007, p.32).

From a Western, particularly American standpoint, the newest era in the history of presenting and explaining war and weapons to the public begins after the Vietnam War. The Vietnam War was a defensive one, fought in difficult terrain against an insurgent enemy - which resulted in increasing reliance on aggressive use of firepower to avoid American casualties (Lewis, 2007, p.261). For those who supported the American effort in Vietnam, its presentation in the media was essentially a public relations disaster, as Andersen (2006) notes: "a majority of the American public turned against the fighting after the Tet Offensive of January 1968 when television imagery revealed the escalating brutality of the war" (xxi-xxii). It remains, to date, associated with the images of human suffering on both sides - the shocking casualty figures sustained by troops, the frequently extreme methods with which the war was conducted, and the devastation and loss endured by the Vietnamese population. The nature of the images seen by the public owed much to the unprecedented access which reporters had to the war: "It was common practice for journalists to sleep in the barracks, eat in mess halls with the troops and talk to the commanding officers as well as local officials and peasants, all of this without public affairs escorts" (Andersen, 2006, p.156). It was clear from these images that technological superiority had not, in fact, eliminated suffering but in many ways 
exacerbated it. The media played an instrumental role in the public opposition to the war in the U.S. and elsewhere, and as Lewis concludes, "The Vietnam War damaged America's ego. The myth of America received a severe blow. [...] Americans believed they, as a people, were morally right and courageous. Vietnam called all this into question" (p.293). Needless to say, the military and political leadership drew its own conclusions from this experience: "In post-Vietnam War operations the Army and the other services, remembering the media's coverage of that war, tightly controlled the movement of the media, limiting its access" (p.371). Public opposition had very concrete and lingering political effects, often described as the 'Vietnam Syndrome' - a deep scepticism and distrust of any military intervention in world affairs by the U.S. (Lewis, 2007). A new approach to presenting the war thus had to be found in order to gain support for any major operations.

In 1990, Iraq invaded Kuwait, sparking an international standoff. With the UN's support, a large coalition was gathered to protest Saddam Hussein's annexation of the country. As negotiations continued without success, military forces from the US and its allies gradually deployed to the Persian Gulf. As the prospect of war loomed, the Western public was inevitably interested in how it would be fought if it happened - and perhaps more importantly, allowed Western media to prepare for covering it when it did happen (Andersen, 2006; Allan and Zelizer, 2004). In the US, military officials were eager to highlight the effectiveness of their forces and weapons, which had received significant technological updates since the Vietnam War, including so-called 'smart weapons', better described as precision-guided munitions such as laser-guided bombs and autonomous cruise missiles that could guide themselves to targets assigned to them from a safe distance (Lewis, 2007). These received great publicity in the press - but from a safe distance from the conflict itself. 
As Andersen (2006) noted, the military carefully managed media involvement in the conflict to avoid a repeat of the coverage in Vietnam: "No independent freedom of access was allowed. Journalists were organized by the military into pools" (p.155). In the coming war, military officials would be working closely with the media to portray the war in the way they wanted it to be seen. The final ingredient for this potent new image of war was inadvertently provided by Saddam Hussein himself - his regime's actions received international condemnation and were widely unpopular throughout the world (Lewis, 2007, p.346). Among the Western public, there would be little trust in any claims about the war's conduct coming from his regime, and little sympathy for the cost his forces would pay should the war start. Although perhaps weary of prospects of a major war, viewers and readers of news in the West would be much more willing to accept and embrace the dominant, official, and largely approving portrayal of the Coalition's war effort.

Operation Desert Storm began on January $16^{\text {th }}, 1991$, after the deadline for Saddam Hussein's withdrawal from Kuwait has passed. From the first hours of the air campaign against lraq, briefings from Pentagon and White House officials provided current information on the war's progress. The war received unprecedented, real-time coverage through 24-hour cable news channels, especially in the US and Britain, which universally saw "marked increases in their audience ratings" (Allan and Zelizer, 2004, p.6). More importantly, official sources provided exclusive footage of air strikes filmed from the airplanes and even weapons themselves - 'smart bombs' and cruise missiles hitting pinpoint targets of military value. The footage was striking and effective, and soon became the focus of the increasingly intense and enthusiastic TV coverage: "The night footage of the bombing of Baghdad was an aesthetic spectacle, the visual reference of fireworks symbolizing the destruction of the city. High- 
technology weaponry became the answer to issues once raised about the moral and ethical - or even economical - problems of war" (Andersen, 2006, p.164).

Official sources declared the strikes successful - and the war lasted a very short period, with Coalition ground troops fighting only several brief engagements by the end of it, with minimum losses, most of them by friendly fire (Lewis, 2007). Most of the campaign was fought in the air, where 'smart bombs' and high-tech aircraft reigned.

Of course in truth the situation was far less straightforward. As Andersen (2006) noted, "The vast majority of airtime was filled with reporting that revolved around a narrowly defined military perspective" (p.188), while other perspectives were sidelined. This view precluded concern for civilians, and the media coverage reflected this: "Utterly absent from media discourse was concern for the people of Iraq. The coverage created the desired impression that this war was accurate, antiseptic and, above all, clean, just like the world presented in commercial messages" (p.178).While 'smart' weapons indeed helped strike many strategic targets, they were a very small percentage of munitions used in the war. There had been errors as well, but as Lewis (2007) suggests, they were sidelined from the media, in part by the distrust toward the Iraqi claims: "Mistakes were made: The Air Force struck a civilian bunker and a prison [...] But, for the most part, Saddam Hussein lost the propaganda weapon he believed was of strategic importance" (p.346). Towards the end of the war, tens of thousands of retreating Iraqi troops are known to have been killed in heavy, indiscriminate bombing on the so-called "Highway of Death" - but coverage of these, as Andersen (2006) emphasized, had been quite one-sided: "One pilot said the road to Basra, filled with the withdrawing Iraqi army, looked like "spring break at Daytona 
Beach" as he bombed the congested line. No images from the ground that might have sobered up the college metaphor were provided" (p.167).

The war was lauded as a success, having been supported by a large coalition and having achieved its goals. It was short, it was effective, and it produced images of weapons that likewise promised a new era in warfare. It may have inflicted a vast amount of suffering, but it was the images of efficiency and precision that dominated coverage in the West. In a sense, 'smart weapons' were the heroes of this war. Some compared the new imagery associated with them to video games (Andersen, 2006). Lewis (2007) summarized the results of the war and its impact on the media as follows: "In Desert Storm Americans could rightly conclude that the Armed Forces performed in an outstanding manner. However, if the press had been given free reign the picture might have been somewhat different. Bad things happen in every war" (p.373). In the eyes of military officials, as Andersen (2006) suggests, the Gulf War provided a cultural model to be followed: "After the First Gulf War, smart bombs and high-tech weapons became the central commodities around which the culture of war revolved" (p.191).

Over the next decade, American and other allied forces remained in the region, periodically conducting operations to enforce sanctions and no-fly zones imposed on Iraq after the war ended. While Iraqi civilians suffered a great deal under the sanctions, and often became 'collateral damage' in strikes, the overwhelming disapproval for Saddam Hussein's regime in the West kept public opinion largely in favour of these sanctions and operations. In 1998 there was yet another brief air campaign against Iraqi military installations - which is what CNN's (1998) documents examined in this study refers to. 
Following the terrorist attacks of September $11^{\text {th }}, 2001$, public outrage in America (and many other Western countries) had a massive effect on public opinion for the first time since Vietnam, support for war was not a matter of how quickly and safely it could be conducted (Lewis, 2007). The US was prepared to lead a coalition to long-term commitments, where destruction of perceived terrorist threats was the primary objective and permanent solutions were sought. Officials, starting with President Bush, used a powerful rhetoric of standing up to aggression and against tyranny in speeches that resembled those of Allied leaders during World War II - the 'Good War' (Andersen, 2006). However the high-tech presentation of war was there to stay. Coverage of the first clash in the War on Terrorism - in Afghanistan - was less extensive and enthusiastic than in previous conflicts, but with popular opinion still reeling from the shock of the September 11 attacks and a seemingly clear objective, this was not a great concern. Initial progress in Afghanistan was quick and the ousting of the Taliban and its replacement by a new regime was widely praised. For the moment, it was causes and goals of the War on Terrorism that preoccupied Western consciousness, not its weapons (Andersen, 2006).

Through 2002, pressure on Iraq was once again increasing. The US accused Saddam Hussein's regime of secretly continuing its program to produce weapons of mass destruction, and raised concern that these weapons may one day fall in the hands of terrorists. Unlike the build-up to the 1991 Gulf War, however, the causes for war were much more controversial and international support for a military solution was minimal (Lewis, 2007). As the prospect of war drew nearer, the media and authorities began preparing for covering the war. Interest in weapons that would be used once again increased. As usual, information on them was released - this time even more extensive, with Andersen (2006) observing that "The "waiting for war" stories that 
dominated coverage were the result of what was being heralded as a new era of military openness" (p.228). Careful to avoid unrealistic expectations, officials and military analysts were now more forward in stating that high technology had its limits and there were instances where it could fail - but would usually work as intended. It was at this stage that the $\mathrm{BBC}(2003)$ published its extensive Fact Files, covering a wide range of weapons and forces that would likely be employed in the upcoming conflict.

Once again Pentagon officials were eager to manage the coverage as well as possible. The war was to be covered live from its very first moments - leading analysts such as Andersen (2006) to draw parallels to the popular entertainment genre of 'reality television'. Nowhere was this more evident than in the newest, albeit lowtech addition to the media arsenal - embedded reporters: "In Operation Iraqi Freedom, the Pentagon modified an old system to fit the new realities of war. It developed a system of embedded reporters, journalists who accompanied units into operations." (Lewis, 2007, p.438) While the concept itself, as Lewis (2007) suggests, is not new, what was unusual was that embedded reporters would be the only journalists able to report from the frontline in the early stages of the ground war. Needless to say, what they could communicate was strictly controlled, in part to avoid endangering the troops, but also no doubt in part to avoid images like those that dominated the public perception of the Vietnam War (p.438). The net result was that when the war began, American and British media would be saturated with information that came predominantly from official sources.

When it did begin in March 2003, the war's first stage featured the cleverlynamed "Shock and Awe" campaign, conceived largely by Donald Rumsfeld who envisioned a comprehensive attack to overwhelm the Iraqi government and military 
structure and dominate the information war (Lewis, 2007, p.414). The ground war was captured by embedded reporters almost exclusively, and proceeded at lightning speed - often with likewise clever names for operations such as the "Thunder Run", the direct tank assault that captured Baghdad in a matter of hours (Lewis, 2007). When major combat operations were announced to have been completed in May 2003, there was no doubt that they succeeded. The main target, Saddam Hussein's Baathist regime, was overthrown - which was precisely the goal of the campaign.

As Lewis (2007) relates, the official doctrine of the Coalition during the war "was based on the premise that the United States was fighting a state, not a nation, and that it was possible to maintain the separation between the people and the government" (p.414). There was probably a fair amount of self-congratulation among military authorities on the handling of the public attention towards the war - they took both positive and negative lessons learned in Vietnam, the Gulf War, and other conflicts such as Kosovo, applying them in the most extensively-covered conflict to date with great success. They produced a tightly-controlled image of the war that was powerful and distant at the same time, and avoided producing unrealistic expectations (Andersen, 2006). While the coverage may not have persuaded opponents of the war, it maintained a solid measure of public support at home. To many, the war was a foregone conclusion, although there was some surprise at just how quickly it had concluded.

Lewis (2007), however, suggests that the view of the war as a guaranteed success is erroneous, and criticizes the much-praised 'Shock and Awe' premise of the war as a serious mistake: "in the case of Operation Iraqi Freedom, Rumsfeld took significant risk [...] had the Republican Guard and Special Republican Guard fought loyally and tenaciously, and had Saddam Hussein's system produced a few good 
generals, they could have made the United States pay dearly for Baghdad. The Rumsfeld Doctrine would not have worked against a unified nation-state fighting a more total war" (p.435). Likewise, Andersen considers the official stance on fighting the state while avoiding the nation to be, at best, unrealistic:

Telling viewers that civilians were not targets and that the Pentagon is concerned about casualties is a comfort. It seems a humane sensibility, but it hides a fundamentally antihumanitarian reality. When an intensive bombing campaign named Shock and Awe is planned, celebrated and carried out in an urban area, how can the deaths of civilians be accidental? (p.261)

Both Lewis' (2007) and Andersen's (2006) analyses show that the war was in fact fraught with difficulties, but none had been severe enough or exposed sufficiently to cause public protest.

What the military officials no doubt underestimated was threats coming from beyond the Baathist regime. Soon after the major combat operations ended, the Coalition became embroiled in an insurgency campaign conducted against it by both Sunni and Shiite militias within Iraq. As of the date of writing, violence continues, and the presence of Coalition troops there remains contentious (Andersen, 2006). Casualty figures, tactical blunders, and civilian suffering are once again increasingly finding their way onto news pages, although unlike the news of victorious results of 2003, these hardly make the front pages, as Lewis (2007) notes: "By the summer of 2005 , the deaths of thirty or forty Iraqis and two or three American soldiers or marines were no longer front-page stories. On the evening news the appointment of judges and the potential of Viagra (a drug for male impotence) to cause blindness trumped the death of a few soldiers and marines" (p.439). As the war lost popularity and clarity, its 'reality show' portrayal all but disappeared. (Andersen, 2006)

In the face of asymmetric warfare, maintaining a positive image has created a difficult public relations challenge for political and military leaders, in the US and 
elsewhere. But while this challenge is yet to be resolved, the lessons learned in handling coverage of large-scale offensive operations will not be forgotten. Tensions are currently looming over Iran's nuclear program - and if they ever spill out into war, there is no doubt that an arsenal of modern, high-tech weapons will once again be used to its full potential. Should this happen, 'smart bombs', cruise missiles, unmanned attack planes and other high-tech systems will yet again be on the front pages of international news.

In more general terms, Lewis (2007) warns that the perception of war being created through the pervasive emphasis on technology is a dangerous one: "The United States is working tenaciously to eliminate man from war, to kill with technology from a safe distance, to fight war without casualties, and in the process of doing so it is losing sight of the objectives of war" (p.399). As he suggests in his analysis, this perception is fundamentally flawed and is in fact undermining American security and capability to engage in war, lulling the public into a false sense of complacency enhanced by an image of war which Andersen (2007) likens to a form of entertainment being propagated through the press. The fact from which this particular culture of war appears to be distancing the Western public is that, according to Lewis (2007), "Technology can augment, expand, and enhance the capabilities of man, but, it cannot replace man. War is ultimately a human endeavor." (Lewis, 2007, p.451)

These conclusions and the recent history of the role of media in war will certainly be used to inform the analysis to some extent, providing some basis to determining ideological antecedents for patterns found within imagery. 


\subsubsection{New Media}

Kress and van Leeuwen (2006) use the term 'new media' to refer primarily to online hypertext media, emphasizing drastic differences between this type of media and its more 'traditional' predecessors, in particular print media: "The world represented visually on the screens of the 'new media' is a differently constructed world to that which had been constructed on the densely printed pages of the print media" (p.31) The rapid development of computer networking technology over the past two decades, has resulted in the rise of a rich hypertext-dominated World Wide Web (or Internet). From a semiotic standpoint, the most significant features of Internet media are its ability to create non-linear texts and networks of texts connected through chains of hyperlinks, and its ability to embed a wide variety of non-text media into text content - including images, audio, video and interactive content such as games (Lemke, 2002). Boczkowski (2004) summarizes the genesis of 'new media' as follows: "online newspapers have emerged by merging print's unidirectional and text-based traditions with networked computing's interactive and (more recently) multimedia potentials. This occurred partly as a reaction to major socioeconomic and technological trends" (p.4)

The emergence of this type of media signals a shift in the role of the readers/viewers - it is now possible for them to become more actively involved in the text, giving them a much greater flexibility in selecting and accessing content, and allowing them to become (inter)actively involved in the text rather than being passive observers (Boczkowski, 2004, p.63). From a purely technical standpoint, today's Internet technology is also far more efficient and far less expensive than print media when it comes to both the volume of text and the saturation of visual content it can provide - which, in theory, makes it the medium with greater multi-modal potential 
than any other (Mautner, 2005). Its interactive nature is further enhanced by the fact that creating, altering or contributing to content online is also very efficient - apart from being simply a type of information media, the Internet is a powerful communication device.

Boczkowski (2004) links the development of online media with the print media's efforts at innovation for the sake of drawing back its falling readership: "In the early 1990s, newspapers began narrowing their exploratory endeavors and, circa 1995, focused on the web as their preferred non-print publishing environment" (p.4). However, he cautions against viewing 'new media' as a fixed, consistent, predictable set of genres:

Most convergence rhetoric has assumed that technical changes would drive all media into a common form regulated by a single logic and has speculated about how best to characterize this product and its social implications. In contrast, my study shows that online newspapers have unfolded by merging print's old ways with the web's new potentials, in an ongoing process in which different combinations of initial conditions and local contingencies have led to divergent trajectories. (p.17)

Online media took on a structure and function that differed greatly from any other available type of public media: "By the end of the 1990s, online newspapers exhibited a technical infrastructure, nascent communication and organizational patterns, and a suite of products that looked very different from those of a typical print counterpart" (p.52) By the mid-1990s, the Internet was a booming network that was saturated with content, much of it visually-rich; was relatively easy to use; and had a sizeable user base, predominantly in the world's most developed countries. It was at this stage that major media corporations had recognized its potential value and began establishing news outlets online. By 1997 both BBC and CNN - as well as many other international news companies - were providing regular news service through the Internet (BBC, 2008; CNN, 2007). As readership increased, so did the amount of 
content designed specifically for their Internet outlets. Among the first such applications were in-depth features that contained technical, political or historical background information relevant to current events that would have been too complex and inefficient to present through other media, including television and print.

The amount and diversity of visual content in online media also has significance when it comes to giving its creators greater capabilities - while the number of images in both sets of documents examined for this study is far from surprising, it is certainly significant and is generally greater than in traditional printed news media. They are also used in ways different from printed media - owing largely to the non-linear structure of web media, and the ability to incorporate images into hyperlink menus and interactive content (Lemke, 2002). More importantly, images often take a dominant role, serving as the core of the discourse and the primary, rather than secondary mode of meaning. As technology allows visuals to be transmitted more efficiently, at less cost and in greater amounts than ever before, Internet media is allowing a more thoroughly visual semiotic to develop, and Mautner (2005) has particularly noted this as one aspect of online discourse which should be considered for CDA research. In the present study, I intend to outline some of the ways in which this visual potential is being applied. 


\subsubsection{CNN and BBC - Background}

The two data sources at the center of this study, CNN and BBC are two of the most influential media corporations in the world, with global reach and a history of involvement with online media.

The BBC was founded in 1922 as the British Broadcasting Company, initially as a radio network. As of today, it runs a global network of television, radio, and online news service - and is reportedly the world's largest broadcaster (BBC, 2008).

Online, the $\mathrm{BBC}$ was first represented by a small website, the $B B C$ Networking Club, in 1994. In December 1997, BBC Online was launched as the company's home page, which increasingly featured news stories. According to BBC, it became "one of the leading websites in Europe by the end of 1999" (BBC, 2008). Later renamed to $B B C i$ and today known as $b b c . c o . u k$, the BBC's online branch is a major online media outlet, featuring a full range of news and other media services, including in-depth features, image galleries, video materials, and round-the-clock updates for news stories. Although the English version of the website is by far the most extensive, a number of localized versions in other languages are offered as well. By 2003, the layout and design of the BBC website attained a format which, with some alterations, it retains today. In $2008, b b c . c o . u k$ underwent a significant technical update, which introduced extensive use of embedded video technology to illustrate its current news articles (BBC, 2008).

The Cable News Network was founded as the world's first 24/7 television news channel in 1980 by media tycoon Ted Turner (CNN, 2007). The format proved immediately successful in the US, and similar cable news channels are now found around the world, including BBC's Newsworld (BBC, 2008). CNN was also an early entry into online news, with its website cnn.com first appearing in August 1995 (CNN, 
2007). The amount of news content on the website increased rapidly, and CNN pioneered many of the features now commonly seen on news websites throughout the world. By 1998, it was already a fairly sophisticated, stand-alone news source, with in-depth content such as the documents on the weapons and forces of the Iraq conflict, which is examined in this study. It has since continued developing and remains a leading online news source (CNN, 2007).

Both $\mathrm{BBC}$ and $\mathrm{CNN}$ covered the conflict in Iraq extensively on their web sites, not only as part of news stories, but also with in-depth features explaining the historical and technological background to the conflict.

\subsubsection{Data Selection - CNN and BBC Documents}

The CNN's (1998) Forces in the Gulf and Bioweapons Explainer are part of a larger feature (set of documents) called Strike on Iraq, which besides the military and technical information also includes political information, maps of the region, and a timeline of events as they unfolded.

The Forces in the Gulf documents include four major sections: (1) U.S. Military Assets; (2) Iraqi Military Assets; (3) British Military Assets; and (4) America's High Tech Weaponry. The latter provides details on American aircraft and munitions used in strikes against Iraq, the latter including 'Smart Bombs', cruise missiles, and air-to-air missiles. It is worth noting that while there are numerous individual articles on Coalition weapons and forces, only a few pertain to Iraqi weapons (although three of the articles on Iraqi troops are longer than any of the articles on Coalition forces). The Bioweapons Explainer, linked directly from the Forces in the Gulf documents, contains a short explanation of the types of weapons of mass destruction that Iraq was believed to possess. 
While CNN's (1998) documents are technically outdated by this point, they are still linked to in other $\mathrm{CNN}$ articles, other websites such as globalsecurity.org, and can be easily found via Google search using terms such as 'smart weapons'. It is also useful to consider these from a historical standpoint - as an example of internet news media of the late 1990s, a key period of development for 'new media' when many of its unique features were still being formed. In some ways, therefore, CNN's (1998) documents could even be said to be a transitional stage between print and online media - taking only partial advantage of multimodal and non-linear possibilities offered by the medium. It certainly has a less extensive and varied use of images than the much more modern and complex BBC (2003) document.

The BBC's (2003) Military Fact Files are a comprehensive set of documents dedicated to weapons and forces of the Iraq conflict, and are a self-contained special feature that had been published on the eve of the 2003 U.S. war on Iraq in March 2003. It has been updated since, mostly to highlight results that some of the weapon systems and forces attained in the war, but still maintains its format and orientation as a device to familiarize the users of BBC's website with the military aspects of that conflict.

There are six major sections in the Fact Files: (1) Forces, describing both Coalition and Iraqi troop formations; (2) Firepower, describing both Coalition and Iraqi munitions; (3) Land, focusing primarily on Coalition and Iraqi tanks; (4) Sea, describing Coalition ships and submarines; (5) Air, describing Coalition and Iraqi aircraft; and (6) Bio/Chem Weapons, explaining different types of weapons of mass destruction that Iraq was believed to possess. As was noted in reference to CNN's documents above, in all sections except the last, a substantially larger number of articles is dedicated to Coalition forces than Iraqi forces. 
Comparing the CNN (1998) web page, as above, to the BBC (2003) Fact Files on the U.S. war on Iraq one can see that the difference between them in terms of format is primarily an evolutionary one - that is, the overall layout and ways in which they present information are very similar, but the BBC simply has more content, larger and higher-definition images, and a more sophisticated, graphics-rich interface. It includes a few other technically-sophisticated items, including a short animated presentation.

The BBC (2003) Fact Files remain a highly-read document, largely because of BBC's extensive thematic linking, which connects most news articles relevant to the U.S. war on Iraq to 'in-depth' information found in the Fact Files. Some of the articles in it had also been updated since the conclusion of the major military operations in May 2003, reflecting some of the results that units and weapons described in them achieved. The Fact Files are also widely referred to on other websites pertaining to weapons and the U.S. war on Iraq, and is readily found on Google search under a wide range of terms (including names of many of the weapons described in the documents).

\subsubsection{Data Organization}

For this study, I have analyzed a total of 177 images - which includes all of the illustrations directly contained in the articles pertaining to weapons and forces of the U.S. war on Iraq, a total of 40 from CNN's (1998) documents and 137 from BBC's (2003) Fact Files. The CNN and BBC images are approached separately in the analysis, and while they are compared and contrasted against each other where applicable, samples from both sources are presented in Chapter 4 and the Appendix as far as possible - since there is quite a gap in terms of the number of images, 
publication date, intended audience and technological resources between the two sources.

The first challenge in dealing with the data was one of organization - which was approached in several ways in this study. The first and most obvious is categorizing the data by topic - with the CNN (1998) and BBC (2003) documents themselves organized into a number of sections, covering topics including aircraft, ships, munitions and troops. For the purposes of this study, these were reduced to five sections in total:

\section{Section I - Coalition Vehicles}

Articles on aircraft, naval ships, land combat vehicles (e.g. tanks)

\section{Section II - Coalition Troops}

Articles on troop formations and types

\section{Section III - Iraqi Troops and Equipment}

Articles on all Iraqi military units and weapons (except biological/chemical)

\section{Section IV - Coalition Weapons}

Articles on munitions used by Coalition troops and vehicles

\section{Section V - Biological and Chemical Weapons}

Articles on Iraq's alleged weapons of mass destruction

The analysis presented in Chapter 4 discusses each of these topic sections individually, noting patterns within and among both.

The second set of categories used in the data was built around generic types found in the data. In the Analysis chapter, I outline eight types of images based on surface features. All of the images in the data are categorized into one of these types, with the distribution of genres used for analytical purposes. For discussion of these genres, refer to Section 4.1.1 in the following chapter.

Thirdly, the images are categorized based on their function in relationship to the text of the articles in which they appear - which is based on their position and 
salience, relating to informational structure (Kress and van Leeuwen, 2006) as

outlined earlier. This categorization is presented and explained in Chapter 4, Section 4.1.2.

Using the above three categories, macro-analysis of the distribution of images by topic (section), genre, and function (within article) was conducted on the entire data set (177 images). The findings of this analysis are presented and discussed in Chapter 4.

\subsubsection{Micro-analysis, Narrowing Data Selection}

All of the images within this study (137 from BBC's (2003) Fact Files, 40 from CNN's (1998) documents) were analyzed in detailed during the intial stages of the project, using the analytical framework outlined in Section 3.2.1 above. In presenting the results of this analysis, some concessions have to be made in terms of the amount of data presented - largely out of consideration for print space, as extensive analytical framework had generated approximately 255 pages of raw data analysis for the entire set of 177 images.

As the primary focus of this research is to uncover patterns and describe them in primarily qualitative terms, a smaller sample of images comprising a total of 42 illustrations was drawn for presentation in this paper, and can be found in the Appendix. The micro-analysis component in Chapter 4 is based primarily on this narrower sample.

The selection of these images was neither arbitrary nor random - they have been chosen based on the observations made in the entire sample to represent major patterns observed through detailed functional analysis, as well as salient exceptions and variations wherever possible. While there are certainly flaws inherent to this 
method from the quantitative perspective, as well as a risk of omitting some of the finer details and variations within the data, from a practical point of view it does offer a way to present the data in qualitative richness and to explain some of the dynamics of visual discourse and describe multiple, complimentary strategies used by BBC (2003) and CNN (1999) in their documents overall. Wherever possible, exceptions and irregularities are discussed and qualifications are made. The distribution of images by genre and function drawn from the macro-analysis, which clearly shows the predominance of certain image types over others, is used wherever possible to support findings on the micro-analysis level. While I acknowledge the limits of this approach, it does allow a fairly efficient presentation of findings based on what is still a substantial data set.

This chapter has thus outlined the methodological approach, context, and data selection examined in this study. I will now proceed to a presentation of the findings, in Chapter 4 below. 


\section{Analysis}

In this chapter, I will apply the analytical framework established in Chapter 3 to the data gathered from the BBC (2003) and CNN (1998) document sets on the weapons and forces of the U.S. war on Iraq, observing firstly the higher-level patterns of genres and functions of images, and secondly the representational, orientational and organizational structures in images as part of overall depiction strategies used in portraying weapons and forces.

\subsection{Macro-Analysis}

\subsubsection{Genres}

Before proceeding to discussion of sample results and patterns found in the images of BBC (2003) and CNN (1998) documents overall, it is necessary to present a brief classification of generic types of images found in the data. The categories below were used during data coding to classify each of the images, and were derived through considering distinctive features, differences in coding orientation, and discursive functions that the images were intended to perform.

\subsubsection{Ordinary Photograph}

For the purposes of this study, the category of ordinary photographs encompasses images which fall within technical norms of modern photography, and have been taken in ways which can be said to represent a naturalistic, ordinary perspective. This is not to suggest anything about their subjects or actual origins, but rather their compositional nature. These images do not use extreme angles, elaborate posing, and do not exceed limits of naturalistic modality - they are made to appear as incidental views of their subjects that would be seen by unprivileged observers, taken without waiting for a particular moment or angle, and often without precise framing of their 
subjects. Most typically, these images are taken from approximately eye level and in colour that is presented in relatively low saturation, which enhances their realism. Below are two typical examples:

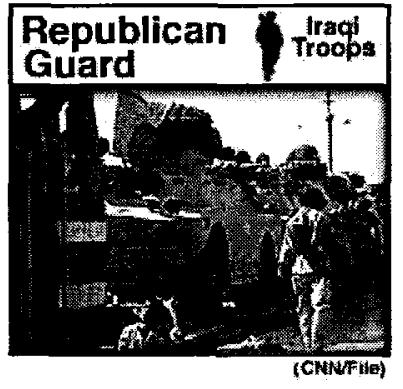

$(\mathrm{CNN})$

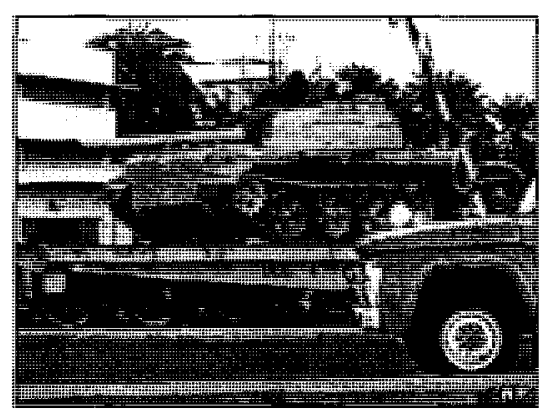

$(\mathrm{BBC})$

Note that both images are taken from approximately eye level and are reproduced in colour with low (but naturalistic-looking) saturation; they do not involve any apparent posing by participants and appear to be made by an uninvolved observer, with only partial view of their main subjects, while there is significant 'clutter' both in the foreground and background preventing complete clarity. The overall implication is that these views are incidental and are made from an unprivileged, relatively distant position. The importance of these features should become more apparent in contrast to publicity photographs.

One way to summarize these properties is to say that ordinary photographs use a naturalistic coding orientation, relying on realism (within the limits of photographic technology) of their depiction to achieve their interpersonal effects. This will be discussed in more detail below.

Within the overall sample, these images appear relatively frequently. In the CNN documents, there are 10 ordinary photographs, which is $25 \%$ of all images used. $\mathrm{BBC}$ contains 26 , which is $19 \%$ of all images used. Both $\mathrm{BBC}$ and $\mathrm{CNN}$ use this type of imagery most frequently when showing Iraqi military equipment and soldiers - one 
half of all images of Iraqi soldiers and equipment can be classed as ordinary photographs. The predominance of these images is not high among images of friendly equipment, however are more common on $\mathrm{BBC}$ than $\mathrm{CNN}$.

\subsubsection{Publicity Photograph}

The primary difference between the previous category and the current one is one of modality - whereas ordinary photographs generally present views that are merely realistic, images in this category go further to present dramatic, elaborate views of their subjects that endow them with symbolism and significance that more ordinary views would not provide. Although still using a medium that is generally considered naturalistic (photography), the overriding coding orientation in these images is a sensory one - where norms of realism for the technology can be exceeded. This type of imagery articulates colours, lighting and contrast to a significant degree in order to make the subjects appear aesthetically appealing and dramatic. This also involves highly articulated perspective - images taken from very unusual angles, in carefully selected poses, using wide or narrow lenses, from points of view that would not be accessible to a common observer or represent a highly naturalistic view, positions which could be said to be 'privileged'.

In public discourse, these are the types of images associated with professional photography, ones which could for instance be used in advertisement due to their striking and symbolic nature. They are used not merely to portray, but in effect to promote. This is a very important distinction, one which is very relevant in this context. In theoretical terms, the key to these depictions is the use of sensory, rather than naturalistic, coding orientations foremost to achieve their orientational effects on the viewer - the quality and aesthetic appeal of these images override realism, and 
often in a way which Kress and van Leeuwen (2006) refer to as "more than real" (p.163) - exceeding norms of modality for the medium.

Two examples are presented below:

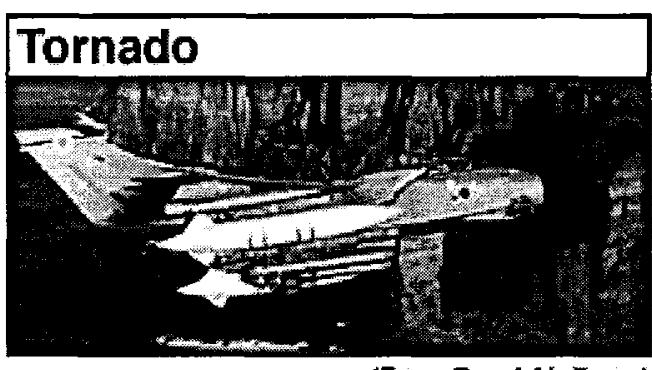

(From Foysal Alr Force)

$(\mathrm{CNN})$

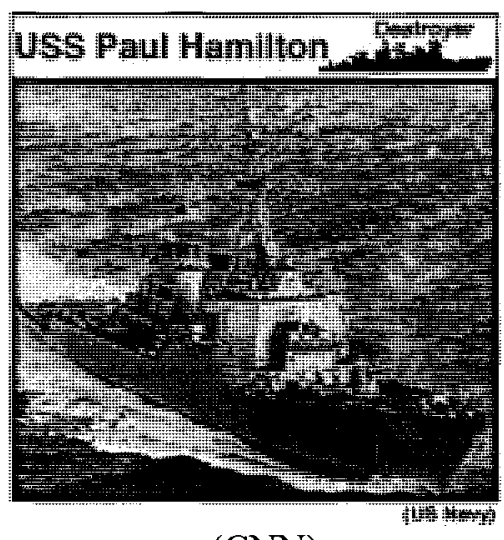

$(\mathrm{CNN})$

Note the extreme high angles that could only be attained by taking photographs from an aircraft; the dramatic horizontal angles, bright colours in the background and symbolic background in the first photo. The power, capability and strongly-defined, diagonal shapes of the subjects are emphasized. These images show neither perspectives that would be typically be accessible by observers or participants in the war, nor provide much information about function, location, and other aspects of these weapons - instead focusing on aesthetic appeal. Although they are by all means realistic portrayals of their subject, they are articulated in a way that goes beyond naturalism. The effects of highly articulated perspective and modality in aestheticallyoriented portrayals will be discussed further below.

Within this study, publicity photographs can be found in both the CNN and BBC documents, with 27 images of this type on CNN (which is $67.5 \%$ of total) and 25 on BBC (18\% of total) - although this number does not include images that combine publicity photographs with other visual elements (see "Combination" below). These images are most prominent on CNN where they are the majority of illustrations 
accompanying articles about Coalition forces, vehicles and weapons ( $81 \%$ of all images pertaining to these subjects).

\subsubsection{Press Collage}

Press collages are most frequently found on title pages, including those of both the $\mathrm{CNN}$ and $\mathrm{BBC}$ documents on weapons and forces. They generally feature combinations of visual elements, most frequently photographs, although perspective and naturalism are only of marginal importance - rather, these are focused on symbolically-relevant representation, showing multiple subjects or participants in either the same or separate frames, suggesting which elements may 'belong' together or which elements may be most salient and symbolic of a subject. They can thus be said to be primarily sensory in terms of coding orientation. Highly-articulated usage of colour, contrast and irregular framing that largely omits or replaces backgrounds are some of the more common features, used most typically to draw connections or distinctions between the various elements brought together in the collage. Individual elements within the collages are rarely involved in overt interactions, and they generally have little role beyond their symbolic significance - and thus are represented in lower levels of detail than they would be in photographs (ordinary or publicity, as above). Rather, they often serve as previews for content seen elsewhere, and often incorporate parts of other illustrations found within a document.

In the BBC (2003) and CNN (1998) documents, this genre is most commonly used for section or feature titles, as seen below. 


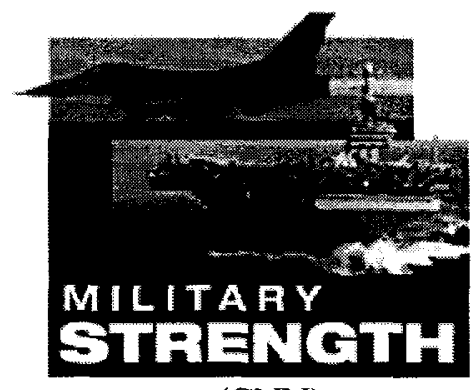

(CNN)

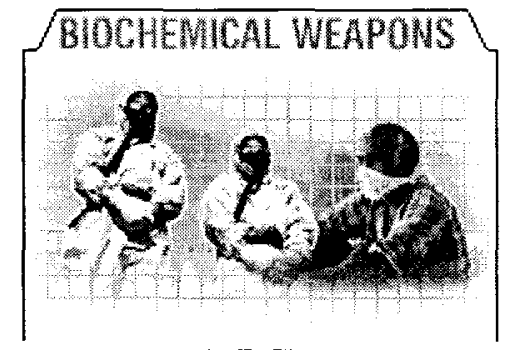

$(\mathrm{BBC})$

It should be noted that not all images categorized as "press collages" featured multiple photographic elements combined. Some included only one, in addition to titles and/or abstract backgrounds, but otherwise shared the 'cut out' appearance and other features. Likewise, not all elements with multiple frames from different types of sources were placed in this category - this will be discussed in the "combination" section below.

Press collages appear only in 2 instances on CNN ( $5 \%$ of total), both times as title images; and 9 times on $\mathrm{BBC}(6.5 \%$ of total), primarily as titles as well.

\subsubsection{Scale Drawing}

Scale diagrams belong to a modality that is oriented towards neither representative realism nor aesthetic effect. These images belong to technological coding orientations - where detail, colour, perspective or depth are only important insofar as they contribute to the representation of structure, scale and/or function of the subject. How they correlate to real appearance, or what symbolic connotations they produce is not considered particularly relevant. They can thus be said to use a primarily technological coding orientation.

Within the data examined for this study, these types of diagrams are found only on BBC's website where they show internal and external detail of weapon systems. They are by no means visually impoverished and have very clean, sharp 
appearance, appealing colours, and usually a measure of perspective. They also usually feature cutaways and labels showing components of these weapons. Some measure of geometric and colour symbolism is used in these diagrams, but their primary focus is on topographic accuracy - showing scale and composition.

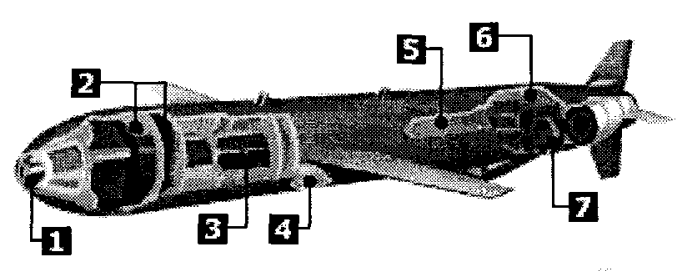

$(\mathrm{BBC})$

There are 8 scale drawings (not counting instances where they are combined with other elements, see "Combination" below) contained in BBC's (2003) Fact Files.

\subsubsection{Function Diagram}

Likewise found mainly in BBC's "Fact Files" in the course of this study, function diagrams are similar to the above-mentioned scale drawings in style and orientation towards function over appearance. They also employ a technological coding orientation. The main difference is that these diagrams are not to scale - rather than topographic accuracy, their primary goal is topological accuracy - accurately showing relations between components and steps in processes such as delivery of weapons. Unlike scale drawings which present structure of weapons systems, function diagrams show stages in material processes in abstract ways. As a result, geometric and temporal symbolism, vectors and visual salience of dominant participants are usually prominently featured. 


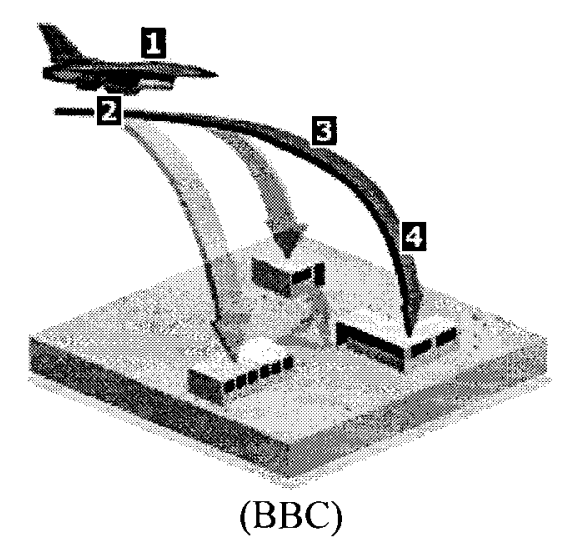

Not including instances where they are used in combination with other elements (see

“Combination", below), function diagrams appear in 19 instances in BBC's (2003)

Fact Files (14\% of total). In the CNN's (1998) documents, the single diagram of the Iraqi command structure can also be considered a function diagram.

\subsubsection{Map}

Geopolitical maps are used occasionally to help situate some of the weapons and emphasize their capabilities. Maps are topographical structures, and their composition is primarily determined by their functional role.

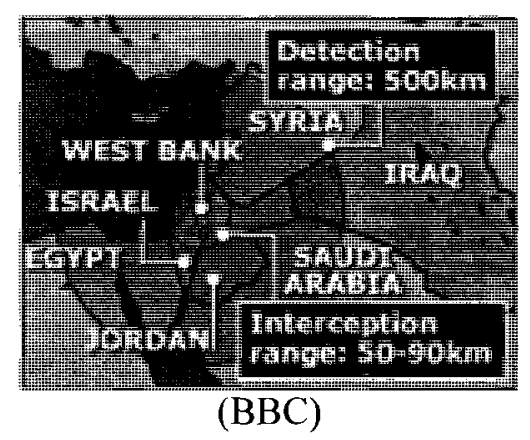

Only one map (shown above) appears in the data, presented on BBC in an article describing Israeli missile defense systems. 


\subsubsection{Optical Sensor Data}

Although found only once in this study, as part of a BBC article on "Smart Weapons", this type of imagery is often considered iconic of modern warfare. It is essentially photographs created from optical sensors carried on weapons and weapon systems, such as 'smart bombs' which rely on such sensors for precise targeting. Due to the nature of the sensors and the extreme conditions and distances with which they have to cope, imagery produced by them is extremely grainy, low-quality, and sometimes difficult to decipher. Furthermore it is sometimes censored to avoid leaks of sensitive military information.

In the media, it is used largely for aesthetic effect, to give concrete examples of the capabilities of the weapons, which are most usually seen tracking very small from long distances, and striking them accurately. While they are certainly naturalistic within the norms of imaging technology from which they are sourced, their use in media contexts often emphasizes their sensory dimensions. The extreme contrasts, electronic text and items like crosshairs are used to draw attention and symbolize the high-tech nature of the weapons. To viewers not familiar with this technology, this imagery provides little to no information value. It is frequently accompanied by explanations that clarify the significance of the images. In the case of $\mathrm{BBC}$, the image below appears in the "Smart Weapons" section and is followed by a series of function diagrams explaining processes that this image is a part of. 


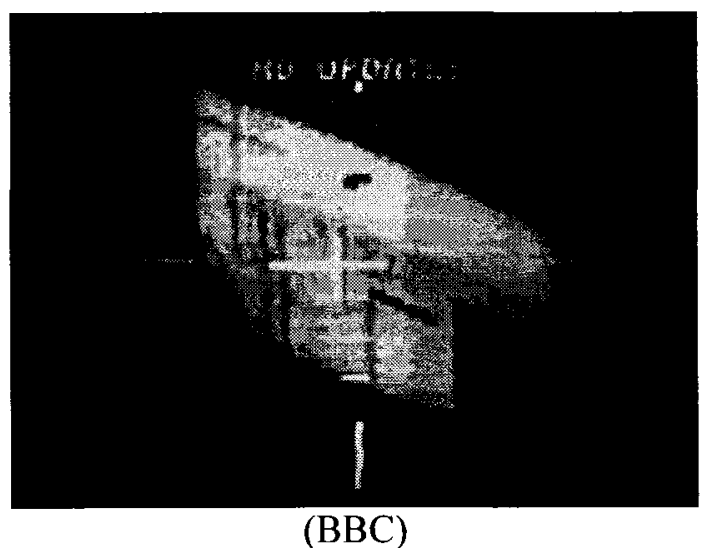

\subsubsection{Combination}

As already noted above, categorizing certain images into generic types sometimes proved challenging. In some cases, combinations of different genres were found in the same space.

For example, BBC (2003) used the layout seen below for many key illustrations in its Fact Files:

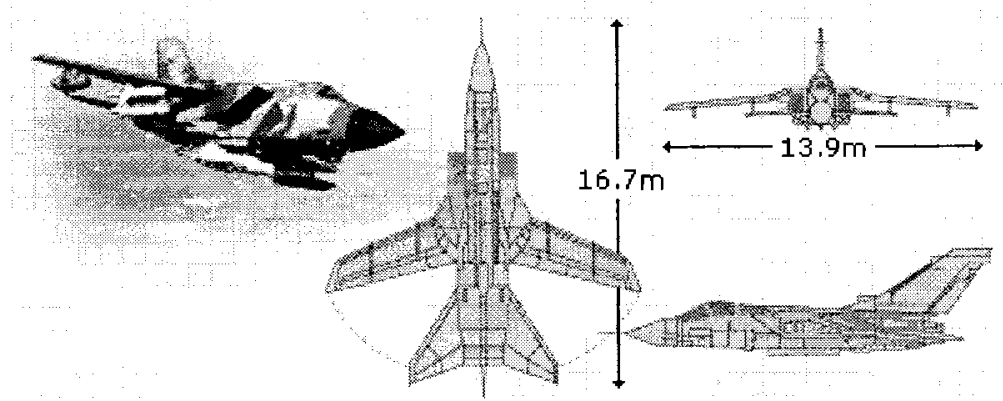

(BBC 97)

On the surface, one could consider this a "press collage", as it does fit many aspects of the category - and even shares the background with some of BBC's collages, but the purpose here is distinctly different. This image is perhaps more accurately analyzed if it's considered to include two distinct elements - a publicity photo element with a faded background on the left, and a scale drawing on the right. Rather than a press collage where the purpose is primarily to create a mood, serving 
an aesthetic purpose, there is informational value in the scale drawing, which is topographically accurate and complements the photograph.

This is a very predominant category on $\mathrm{BBC}$, and with only a few exceptions, the images that comprise it are exactly of the same structure and genre as shown above - a combination of publicity photograph(s) and scale drawing(s), with faded background for the photographs and a light grey grid forming the primary frame of the image. The total number of images placed in this category is 48 , which is $35 \%$ of total for BBC's visuals. Of these, 43 are a combination of photographs and scale drawings presented in a format identical to the example shown above. Of the remaining five, four are also combinations of photographs and function diagrams or scale drawings, used to complementary effect. One is an animation which combines scale drawing and function diagrams in relatively equal proportion.

\subsubsection{Summary}

The images found in the data sample thus fall into eight different categories, most (but not all) of which use photographic sources in some form. The distribution of images by generic type is summarized in the charts below:

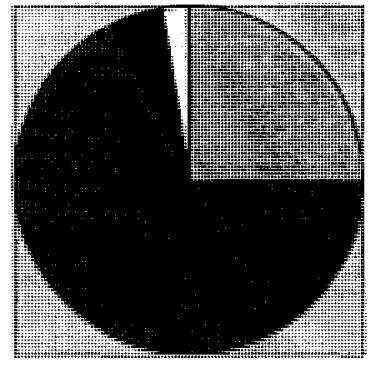

CNN - 40 images

\begin{tabular}{|l|}
\hline Oag Ordinary \\
Photo \\
Publicity \\
Photo \\
Press \\
Collage \\
Function \\
Diagram \\
\hline
\end{tabular}

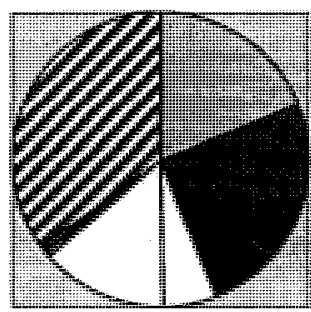

$B B C-137$ images

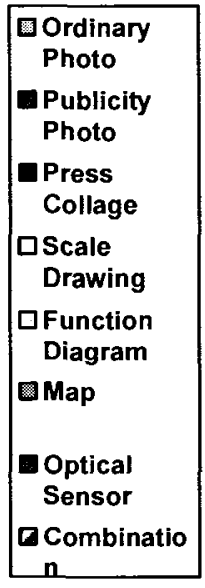

n 
As this analysis suggests, there is a significant difference between $\mathrm{BBC}$ and $\mathrm{CNN}$ in terms of the distribution of different types of imagery. Where this difference is most apparent is in scale drawings, function diagrams, and the "Combination" category which generally includes scale drawings and diagrams. These comprise a significant part of the sample in the BBC's (2003) Fact Files, yet are virtually absent in CNN's (1998) documents. By contrast, CNN's documents use publicity photos in the overwhelming majority of articles.

The implications of this distinction will be discussed further below; at this point, it is sufficient to say that there is significant strategic difference between the way $\mathrm{CNN}$ and $\mathrm{BBC}$ present the same type of discourse.

\subsubsection{Functions}

In terms of the role of images in combination with the text, as part of larger multimodal documents, I would distinguish three primary categories into which use of images would fall:

\subsubsection{Primary illustration}

Primary illustrations are often the sole images within an individual article, and serve as summaries of the overall 'essence' of their subject. They usually portray the subject either in full or in a detailed close-up, and normally present an aesthetically-refined depiction that reflects the subject's qualities. Primary illustrations are usually located at the start of the articles and one of their primary roles, in conjunction with article titles, is setting the theme or mood within their respective articles. Due to their default position at the top of the page, above the main text body within an article, they are presented as the ideal - the generalized essence of the discourse, as opposed to the 
text which is presented as the real - concrete facts and practical information. Typically, publicity photos are used in this illustrative role.

CNN uses these extensively within their documents on weapons and forces.

\subsubsection{Secondary Illustration}

As opposed to primary illustrations which try to summarize their subject in one image, secondary illustrations only show individual features of the subject, aspects of its use, or the context in which it is usually found, referring to text or other images for further detail and synthesis. This type of illustration includes both ordinary and publicity photos. These images are usually located somewhere within the body of the article, rather than at the top.

BBC's (2003) Fact Files use these extensively in conjunction with subtitles that relate the images to text articles. In most instances where secondary illustrations are found on $\mathrm{BBC}$, they are located to the right of the main text body, which places them in a given-new relation with the text, which serves both to solidify the factual nature of the text and underline the role of images as giving additional information on specific, singular features of the subject rather than presenting its generalized essence.

\subsubsection{Exposition / Explanation}

This use of images coincides largely with scale drawings and function diagrams. Rather than summarizing qualities or presenting individual features of the subject, they provide a more concrete representation that is largely technical in nature. In the case of scale drawings, this involves exposition of the subject's components and dimensions - not qualities, or at least not overtly; in the case of function diagrams, complete explanation of its functions - not a snapshot of an aspect within them. By 
default, these images are exhaustive - that is, they show a complete set of attributes, relations and/or functions. This is generally done with the aid of labels, lines, and symbolic vectors such as arrows - though it can be accomplished without these, for example by creating very clear covert taxonomies (by showing multiple subjects in one frame and symbolising the relation between them through size and position) or using carefully-arranged temporal structures (suggesting sequences in time, through positioning, repetition or symbolism). Photographs can appear in an exposition/explanation role, however this is relatively unusual - primarily because the default mode, technical drawings, lends itself much more readily to these functions and is much easier to articulate and control for attributes and functions on display.

Images placed in this role are usually dominant within their respective articles and contain information that is necessary for their overall interpretation. Since CNN's (1998) articles do not contain such diagrams (with one exception), this use of images is found mainly in BBC's (2003) Fact Files within this study.

Another notable feature of the exposition/explanation role is that it takes on images that combine multiple frames, often of different generic types (see Combination in the discussion of generic types), for example small photo insets within diagrams. In effect, these diagrams often gains their own primary and secondary illustrations - which is not surprising, considering the dominant role these diagrams take within (and often over) the articles. For the purposes of the study, these subordinate illustrations will not be treated separately, but analyzed as part of the larger images in which they are embedded. 


\subsubsection{Summary}

Thus, for the purposes of this study there are 8 generic types of images and 3 patterns of their use distinguished. The overall distribution of these between CNN's (1998) and BBC's (2003) documents on weapons and forces in the Iraq conflict is summarized in the table below:

\begin{tabular}{|c|c|c|c|c|}
\hline & Total & $\begin{array}{l}\text { Primary } \\
\text { Illustration }\end{array}$ & $\begin{array}{l}\text { Secondary } \\
\text { Illustration }\end{array}$ & $\begin{array}{l}\text { Exposition / } \\
\text { Explanation }\end{array}$ \\
\hline CNN & 40 & 38 & 1 & 1 \\
\hline - Ordinary Photograph & 10 & 10 & & \\
\hline - $\quad$ Publicity Photograph & 27 & 26 & 1 & \\
\hline - $\quad$ Press Collage & 2 & 2 & & \\
\hline - $\quad$ Scale Drawing & & & & \\
\hline - Function diagram & 1 & & & 1 \\
\hline - Map & & & & \\
\hline - Optical Sensor Data & & & & \\
\hline - Combination & & & & \\
\hline BBC & 137 & 56 & 53 & 28 \\
\hline - Ordinary Photograph & 26 & & 26 & \\
\hline - $\quad$ Publicity Photograph & 25 & 3 & 22 & \\
\hline - $\quad$ Press Collage & 9 & 7 & 2 & \\
\hline - Scale Drawing & 8 & 1 & 1 & 6 \\
\hline - Function diagram & 19 & 1 & 1 & 17 \\
\hline - Map & 1 & & 1 & \\
\hline - Optical Sensor Data & 1 & 1 & & \\
\hline - Combination & 48 & 43 & & 5 \\
\hline
\end{tabular}

As far as the use of images, once again BBC shows a much wider range, with a notable amount of secondary illustrations and explanation/exposition visuals which are by and large absent from CNN's (1998) set of documents. 


\subsection{Micro-Analysis}

The remainder of this chapter will present detailed analysis of individual images, discussing patterns found within each of the sections and their relevance to the overall effect of the CNN (1998) and BBC (2003) documents. The analysis follows from the framework established in Chapter 3, and discusses Representational, Orientational and Organizational aspects of images found within each section.

\subsection{Section I - Coalition Vehicles}

The first section of analyzed data covers images found in articles about military ships, tanks, and aircraft. These fall into several sections within both the CNN (1998) and $\mathrm{BBC}$ (2003) documents, but the pattern of representation among them is surprisingly consistent. As the table below shows, a single generic type, publicity photograph, predominates in the CNN's (1998) articles - while combination imagery is predominant in the BBC (2003) Fact Files on ships, tanks and aircraft. In regard to the latter, it should be taken into account that all 40 combinations in this sample belong to a specific format that includes publicity photo elements and scale drawings. The format will be detailed further below.

Section I - Coalition Vehicles

\begin{tabular}{|c|c|c|c|c|}
\hline & Total & $\begin{array}{l}\text { Primary } \\
\text { Illustration }\end{array}$ & $\begin{array}{l}\text { Secondary } \\
\text { Illustration }\end{array}$ & $\begin{array}{l}\text { Exposition / } \\
\text { Explanation }\end{array}$ \\
\hline CNN & 24 & 24 & & \\
\hline $\begin{array}{ll}- & \text { Ordinary } \\
& \text { Photograph } \\
\end{array}$ & 4 & 4 & & \\
\hline $\begin{array}{ll}- & \text { Publicity } \\
& \text { Photograph }\end{array}$ & 20 & 20 & & \\
\hline $\mathrm{BBC}$ & 47 & 40 & 5 & 2 \\
\hline $\begin{array}{ll}- & \text { Ordinary } \\
& \text { Photograph } \\
\end{array}$ & 3 & & 3 & \\
\hline $\begin{array}{ll}\text { - } & \text { Publicity } \\
& \text { Photograph }\end{array}$ & 1 & & 1 & \\
\hline $\begin{array}{l}\text { - Scale Drawing / } \\
\text { Diagram }\end{array}$ & 1 & & 1 & \\
\hline $\begin{array}{ll}\text { - } & \text { Function } \\
& \text { Diagram } \\
\end{array}$ & 2 & & & 2 \\
\hline - Combination & 40 & 40 & & \\
\hline
\end{tabular}


Given this distribution, as well as significant similarity among images belonging to the dominant types, further discussion in this section focuses on unpacking the features of publicity photo and combination imagery and outlining patterns between and within them. Photographic imagery that that has differences from the dominant pattern is also noted, but analysis of scale drawings and function diagrams is omitted from the present section - for discussion of these, see the Coalition Weapons section further below.

The most important patterns in this section are as follows:

- Organizational

O Use of primary illustrations - placement above the text; ideal-real relationship with text

- Use of framing, salience, central position to emphasize subjects (CNN)

- Limited internal framing between different representations of same subject (BBC)

- Representational

- Single, inanimate participants

- Non-transactional material actions

o Relational processes, emphasis on attributes

- Limited articulation of circumstances

- Orientational

O Medium distance

- Predominance of high, mostly frontal angles

- Very high articulation of depth of perspective

- High articulation of contrast, colour, lighting

A complete functional analysis of 14 images from this section, 6 from CNN's (1998) documents and 8 from BBC's (2003) Fact Files, can be found in the Appendix.

Results of this sample analysis will be used to inform the specific findings presented below.

\subsubsection{Organizational}

Images found within this section share many organizational similarities; in fact the $\mathrm{BBC}$ and $\mathrm{CNN}$ each have a format which they consistently follow and which extends to virtually all primary illustrations of military equipment. 


\subsubsection{CNN}

CNN (1998) uses the layout presented below. Note that the image is dominated by a publicity photograph (even though it does possess other elements, such as a small scale drawing), and therefore was coded as such.

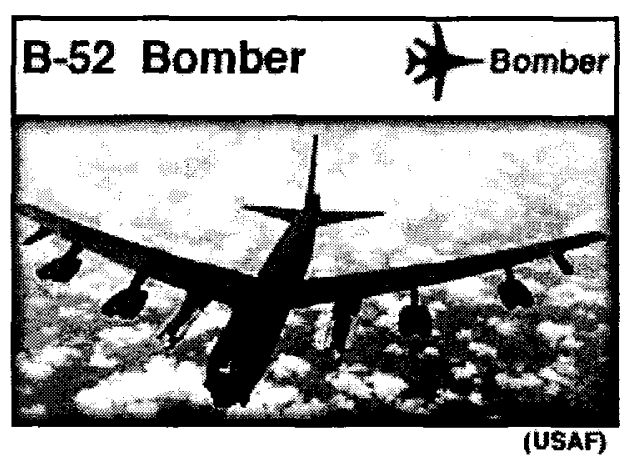

(13) $\mathrm{CNN}$

The image is composed of two primary frames: a black border surrounding the title and a small silhouette of an aircraft in the top part, and a black inward gradient border surrounding the large publicity photo at bottom. Within this photo frame, the aircraft is the most salient element - which is established through size and contrast primarily, and its central positioning in the frame focuses the viewer's attention on it.

Serving as the primary illustration for its respective article, the image clearly establishes a pattern of reference to it, which is further reinforced by the title being contained within the image, labelling it as showing the same subject as the article. The image, positioned directly above the article's text itself, helps establish the overall theme - which becomes more significant when the representational and orientational dimensions for it are considered, since the image helps cement the participants and processes of the discourse, and a certain attitude toward the discourse, which are echoed in the text. These will be discussed further below.

Another way to describe the vertical relationship between the image and the text is in terms of information structure - ideal and real. The image becomes the ideal 
representation - something that is certainly expected of a publicity photo, which provides a visual sense of the subject but not much in the way of concrete facts; and the text, providing specific technical and contextual information (such as how old the aircraft is, where and how it has been used), becomes the real. In this way the text and visual significantly complement each other.

Naturally, the two frames within the image can also be considered to be in an ideal-real relationship: with the title, label and silhouette serving as ideal, alluding to the general idea of a bomber a viewer may have; and the photograph showing the real, relatively naturalistic appearance of an actual bomber.

The final organisational element, which is very difficult to precisely specify, is the relation of the image to public perceptions and popular images. Certainly, an image of aircraft or an allusion to a bomber brings up a whole chain of references. Sometimes these associations are spelled out directly - BBC's article for example refers to this same aircraft as "the B-52 of Dr Strangelove fame". But even without explicit reference, images and articles are bound to engage such associations. While it may be the role of the CNN (1998) and BBC (2003) documents to give information about weapons to the viewer, it is certain that most viewers will have associations with much of the imagery they see already. It would be wrong to assume that the media treats these viewers as ignorant of the subject - rather, it is most likely they rely on existing associations and seek to direct them in favourable ways. In organizational terms, one can at least assume that the viewers' ability to at least recognize the subject as a large aircraft and perhaps a bomber would be assumed and thus, on the one hand, free the authors from having to specify what this entails - and on the other hand, give them a greater latitude in manipulating features of the image such as perspective, lighting and colour while retaining recognizability of the subject. 
This pattern certainly applies to all subjects presented by CNN in this particular fashion.

\subsubsection{BBC}

Like CNN's (1998) images of military vehicles, the vast majority of all images presented in BBC's (2003) sections on this subject are primary illustration featuring an element sourced from publicity photographs. Unlike CNN's images however, this element is not the predominant one - as seen in the photograph above, the primary illustrations of vehicles on BBC use a combination of publicity photograph and scale drawing. The format is consistent throughout, although with variations in the number and positioning of each of the elements - there is always at least one publicity photograph inset and at least one scale drawing, but the number and positioning of these elements in each individual illustration varies. A typical example, containing one photographic element and three scale drawings, all representing the same subject, can be seen below:

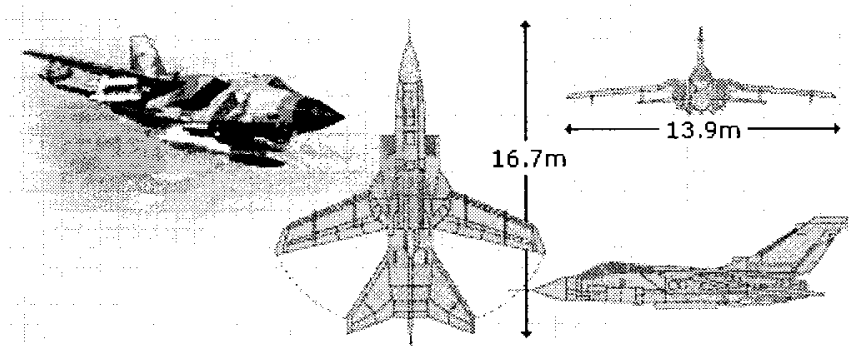

(12) BBC

The primary frame containing the entire image is formed by the light grey grid seen in the background - it is not particularly clear or obtrusive, and thus makes the image blend into the article (which is set against a white background) to a large extent. The internal framing is very vague at best, as in the case of the background around the aircraft fading outward into the same light grey grid; it is non-existent between the 
scale drawings. The intention is to break down the internal borders, in part to make it clear that all the images are of the same exact subject and belong together.

While in this particular image the background grid of the scale drawing extends throughout and the drawings take up much more space, it is the photographic element which remains more salient - in large part because of its high contrast, especially compared to the drawings. The presence of a background, however vague, and productive use of perspective ensure that it is the element that draws most attention. The lower-modality images are peripheral to it, but serve to explain the appearance and features of the subjects in much greater clarity and detail. The use of repetition helps clarify features of the subject and, given the limited internal framing, emphasizes that rather than showing different subjects, all individual elements are united in their presentation of one single (though not individual - but rather generalized) aircraft.

While it is possible to talk about left-right relations within the image, which would make the photo on the left the given element, and the scale drawings the new, other images of military vehicles in this section do not continue the same pattern, and the placement of elements appears largely arbitrary (although it would certainly make sense for a photograph and a scale drawing clarifying what it shows to be in a givennew relationship, thus supporting the role of the image's authors as informationgivers). The relation between the image and the article below it is identical to the pattern seen in primary illustrations by $\mathrm{CNN}$ - the image helps set the theme for the article, and also serves as the ideal to the article's real, thus emphasizing the illustration's role as the "idealized essence of the information" (Kress \& van Leeuwen, 2006) and the text's role as the provider of practical and specific facts. 
Once again, while it may be difficult to trace the references to external realities and popular perceptions that the image seeks to make, it is at least relying on common recognition of the subject as a modern military aircraft.

\subsubsection{Summary}

Thus, the primary illustrations that form the vast majority of visual content in articles on Coalition military vehicles are organized in a way that focuses the viewer on the appearance and external attributes of their subjects. This is accomplished through framing (or within BBC's combination images - lack thereof); salience through position, contrast and use of perspective; and in certain instances - repetition.

The illustrations, by virtue of their vertical position relative to the article, serve firstly to help establish the overall theme for the text that follows them - and secondly serve as the ideal to the text's real, which is a natural and established pattern for primary illustration. Their role is thus to represent the overall essence of the topic.

The images certainly work into a complex network of references, both to specific passages in the text they accompany, and to the outside world and popular perception of their subjects at large. It is difficult to trace precisely what perceptions of their subjects these illustrations may refer to, but it is certainly possible to discuss what perceptions they seek to form through examining representational and orientational aspects of the image, ways in which they construe their subject and orient their viewers. This follows in the discussion directly below. 


\subsubsection{Representational}

Since the most basic functional aim of both the $\mathrm{CNN}$ and $\mathrm{BBC}$ is to present weapon systems and forces, the most dominant mode of accomplishing this goal is to simply show their appearance and features. In most illustrations, this is accomplished by simply presenting the subject in an up-front, decontextualized, largely static fashion. Consider the two examples below in terms of processes, participants and circumstances:

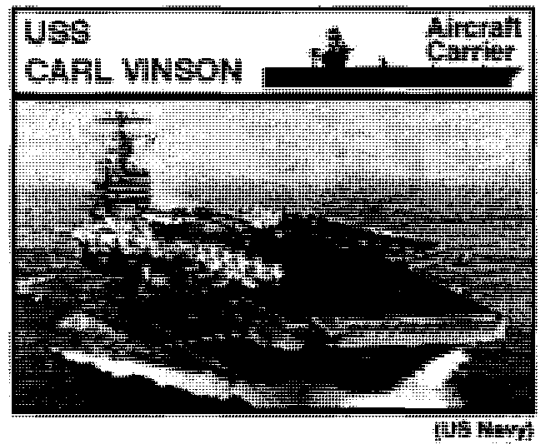

(1) CNN

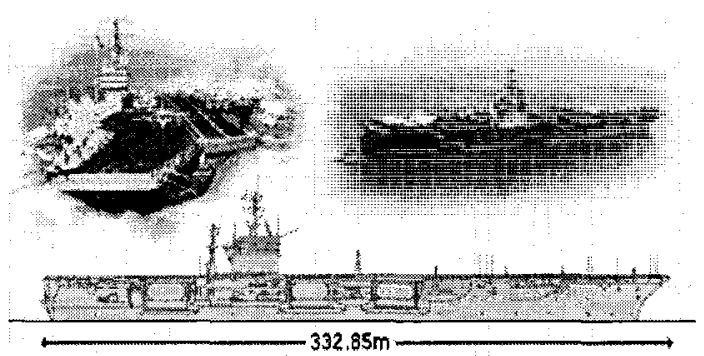

(2) $\mathrm{BBC}$

The subject of both images is the ship, in both cases shown from more than one perspective. The ship can be seen moving in one of the frames within both images, as suggested by the vectors formed by firstly the ship's orientation, and secondly the powerful wave it produces in the water. In the frames where the wave is seen, the vectors are further underlined by the diagonal lines produced by the perspective from which the ship is seen. There are aircraft on the deck of the ship, which could be considered either independent participants or simply attributes of the ship - there is a covert taxonomy suggesting that they are subordinate to the carrier regardless. Perhaps the most accurate way to describe the relation is as a circumstance of accompaniment - linguistically, paralleling the expression "carrier with aircraft". Many other attributes, such as antennas, a flat deck, a square tower on its right side, and so forth, can be distinguished. Besides the sea and, in the first image, the sky, no other circumstances are present. No further information - such as where this ship is, 
where it is going, what it does with the aircraft - is provided. This sort of information can certainly be inferred or gathered from the accompanying text, but it is not provided in the image itself.

The field established in this image corresponds to a large extent with existential, attributive and possessive processes used in linguistic communication the ship is situated in the real world by virtue of the representation and it can be reduced to a series of attributes and components. Since there are no other participants, it is these attributes that become the focus of the image. For all purposes, the image simply shows what the ship is and what it has. In absence of transitivity, sense of time or in fact narrative structure, the sole material action involved also becomes an abstract attribute of sorts - or, again paralleling linguistic analysis, it becomes similar in function to an infinitive structure. Since there is no concrete sense of time or location established (except that it is daytime at sea), and there is no substantial narrative (beyond perhaps "the ship is sailing"), these images can easily be incorporated into other narratives about the subject, which is precisely what happens in this case. This, of course, is usually a property of publicity photographs of military hardware - they are meant to be used and re-used in the press whenever an article involving the subject is written.

One other notable element that is absent in the images is an obvious human presence. Both the $\mathrm{BBC}$ and $\mathrm{CNN}$ articles indicate that a carrier such as the one depicted has a crew of over 5000 people - yet none can be seen in the image, and thus none are participants in the image as such. The ship is an inanimate entity, and certainly it cannot operate without its human crew - but in the image at least, being the only active participant, it certainly does. Thus agency is assigned to an inanimate entity - and certainly the texts accompanying these images bear this out, being 
predominantly about the ship or its components (such as elevators, catapults) performing actions. On the one hand, assigning primary agency and giving primary representation to an inanimate ship is simply a sensible technical choice - it would be difficult to visually characterize a $332.85 \mathrm{~m}$ ship (as BBC's image emphasizes) while giving agency to its human operators who would be at most about $2 \mathrm{~m}$ in their longest dimension; but there is also a significant ideological implication - whether intentionally or not, removing human agency also removes any sense of responsibility for actions the ship may be involved in, along with any personalization of its operators. At the same time this serves to underline the role of technology in conducting warfare - where machines become primary agents. Although in these images the ship is portrayed in a relatively innocuous action of sailing through the seas in these images, its primary purpose is a military one, and it is far from being independent of human agency in reality.

Thus, the images construct a carefully-selected field of discourse, with a single active participant engaged in a non-transactional action, and with only abstract senses of time, place and agency.

In the CNN (1998) and BBC (2003) documents as a whole, this mode of representation is very common for subjects including ships, aircraft and other military hardware, and is especially predominant on CNN. It appears to be a typical pattern for military publicity photos as such.

The observation is confirmed by detailed analysis of the selected data sample. For instance the pattern of representation in fact extends to all images selected in this section: 


\begin{tabular}{|c|c|c|c|}
\hline & Participants & Circumstances & Processes \\
\hline $\begin{array}{l}\text { (1) } \mathrm{CNN} \text { - Aircraft } \\
\text { Carrier }\end{array}$ & Ship, aircraft on deck & Water, sky (location) & Motion (Material) \\
\hline $\begin{array}{l}\text { (2) BBC - Aircraft } \\
\text { Carrier }\end{array}$ & $\begin{array}{l}\text { Ship } \\
2 \text { scale drawings of } \\
\text { same ship }\end{array}$ & $\begin{array}{l}\text { Water (location), grid } \\
\text { (abstract) }\end{array}$ & Motion (Material) \\
\hline (3) CNN - Destroyer & Ship & Water (location) & Motion (Material) \\
\hline (4) $\mathrm{BBC}$ - Destroyer & $\begin{array}{l}\text { Ship } \\
2 \text { scale drawings of } \\
\text { same ship }\end{array}$ & $\begin{array}{l}\text { Water (location), grid } \\
\text { (abstract) }\end{array}$ & Motion (Material) \\
\hline (5) CNN - Submarine & Ship & Water (location) & Motion (Material) \\
\hline (6) BBC - Submarine & $\begin{array}{l}\text { Ship } \\
2 \text { scale drawings of } \\
\text { same ship }\end{array}$ & $\begin{array}{l}\text { Water (location), grid } \\
\text { (abstract) }\end{array}$ & Motion (Material) \\
\hline $\begin{array}{l}\text { (7) BBC - Challenger } \\
\text { Tank }\end{array}$ & Tank & Hills, roads, trees (location) & $\begin{array}{l}\text { Motion, aiming } \\
\text { (material) }\end{array}$ \\
\hline (8) BBC - Abrams Tank & $\begin{array}{l}\text { Tank } \\
2 \text { scale drawings of } \\
\text { same tank }\end{array}$ & $\begin{array}{l}\text { Vehicles (not in correct } \\
\text { perspective), faded; light grey } \\
\text { grid }\end{array}$ & $\begin{array}{l}\text { Forward movement } \\
\text { suggested (material) }\end{array}$ \\
\hline (9) $\mathrm{CNN}$ - F-16 Fighter & Aircraft (F-16) & $\begin{array}{l}\text { Sky, cirrus clouds, mountain } \\
\text { tops (location) }\end{array}$ & Motion (Material) \\
\hline (10) $\mathrm{BBC}-\mathrm{F}-16$ Fighter & $\begin{array}{l}\text { Aircraft } \\
3 \text { scale drawings of } \\
\text { same aircraft }\end{array}$ & Pale grey grid only & $\begin{array}{l}\text { Forward movement } \\
\text { suggested (Material) }\end{array}$ \\
\hline (11) CNN - Tornado & Aircraft & Water, cliffs (location) & Motion (Material) \\
\hline (12) BBC - Tornado & $\begin{array}{l}\text { Aircraft } \\
3 \text { scale drawings of } \\
\text { same aircraft }\end{array}$ & $\begin{array}{l}\text { Pale grey background in } \\
\text { photo, details indistinct; } \\
\text { Pale grey grid }\end{array}$ & $\begin{array}{l}\text { Forward movement } \\
\text { suggested (Material) }\end{array}$ \\
\hline $\begin{array}{l}\text { (13) } \mathrm{CNN}-\mathrm{B}-52 \\
\text { Bomber }\end{array}$ & Aircraft & Clouds, haze (location) & Motion (Material) \\
\hline $\begin{array}{l}\text { (14) BBC - B-52 } \\
\text { Bomber }\end{array}$ & $\begin{array}{l}\text { Aircraft } \\
3 \text { scale drawings of } \\
\text { same aircraft }\end{array}$ & $\begin{array}{l}\text { Pale grey background in } \\
\text { photo, details indistinct; } \\
\text { Pale grey grid }\end{array}$ & $\begin{array}{l}\text { Forward movement } \\
\text { suggested (Material) }\end{array}$ \\
\hline
\end{tabular}

It should be noted that there are some images in the CNN (1998) and BBC (2003)

documents that do not entirely match this pattern. Two exceptions which do include multiple participants, some of which are human, are discussed further below. As far as general patterns however, the representational properties summarized above certainly hold true.

\subsubsection{Orientational}

The orientational structure of the text is most crucial in aligning the viewer, since it is responsible for creating relations within the text - those between the viewer, the subject, and also the authors of the image. With the field defined in relatively narrow 
terms, as discussed above, there is certainly ample room in the images of Coalition vehicles for arranging these interpersonal aspects.

As with other aspects already discussed, there are prevalent orientational patterns in this section. These include perspective and depth, use of detail and sharpness, saturation, contrast and lighting. Consider the features of the photograph below:

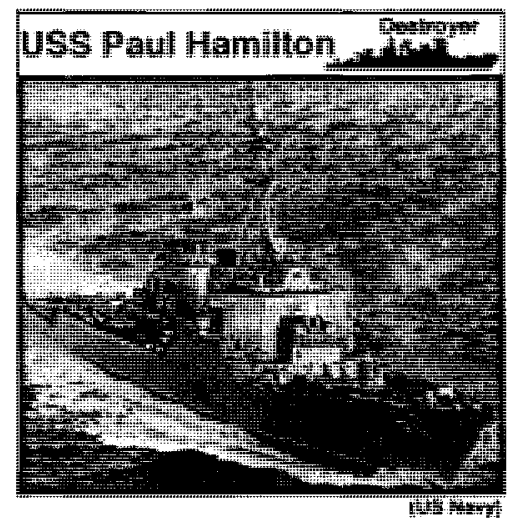

(3) $\mathrm{CNN}$

Firstly, the photograph is framed in a way that shows the entire ship, the edges of which touch the frame but do not exceed it. This is typically described as medium distance, which is close enough to see both the entire appearance of the object and its features as well as possible, but far enough to place the viewer at a reasonable social distance - the viewer, in other words, may be invited to view the ship but is not associated directly with the ship in this way. In terms of horizontal angle, the ship's orientation is approximately $3 / 4$ frontal relative to the viewer - thus, it is approaching the viewer, but not directly. Frontal angles generally indicate engagement - and in this case, it certainly helps to draw attention to the ship and engage the viewer in examining it and its features more closely. In terms of vertical angle, the viewer is placed in a high viewing position, well above the ship. Vertical angle is associated with power, thus the viewer is in a relatively powerful position over the ship, and 
conversely, the ship is not presented in a threatening fashion. There are further implications to this which will be discussed below; before that, the overall relevance of this pattern of perspective should be considered.

The photographic images seen in the BBC illustration below share most features with the image above (medium distance, primarily frontal angle, high vertical angle) - with the exception of vertical angle for the photographic inset on the left.

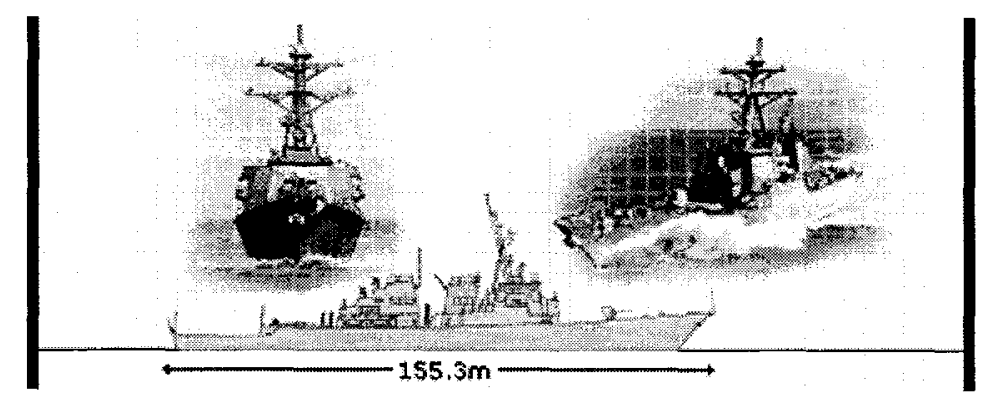

(4) $\mathrm{BBC}$

By virtue of positioning the ship more frontally and at a medium angle (approximately level with horizon), it is made to appear somewhat more threatening but also more capable. Otherwise, the patterns are retained. As the table below shows, there are other similar variations, but the overall mode remains relatively consistent in portrayals of ships and tanks, both in $\mathrm{CNN}$ and $\mathrm{BBC}$ articles: 


\begin{tabular}{|c|c|c|c|}
\hline & $\begin{array}{l}\text { Distance } \\
\text { (relative to frame size) }\end{array}$ & Horizontal angle / facing & Vertical Angle \\
\hline $\begin{array}{l}\text { (1) } \mathrm{CNN} \\
\text { Aircraft Carrier }\end{array}$ & Medium & Frontal (slightly offset) & Moderate-high \\
\hline (2) BBC Aircraft Carrier & Medium-long & $\begin{array}{l}\text { 1) Frontal (slightly } \\
\text { offset) } \\
\text { 2) Oblique (1/3 back), } \\
\text { facing right }\end{array}$ & $\begin{array}{l}\text { 1) Moderate- high } \\
\text { (above deck) } \\
\text { 2) High }\end{array}$ \\
\hline (3) CNN Destroyer & Medium & $3 / 4$ frontal & High \\
\hline (4) BBC Destroyer & Medium & $\begin{array}{l}\text { 1) Frontal } \\
\text { 2) } 3 / 4 \text { frontal }\end{array}$ & $\begin{array}{l}\text { I) Medium (deck level) } \\
\text { 2) High }\end{array}$ \\
\hline (5) CNN Submarine & Medium-close & Frontal, slightly offset & $\begin{array}{l}\text { Very high (almost } \\
\text { vertical) }\end{array}$ \\
\hline (6) BBC Submarine & Medium & $\begin{array}{l}\text { 1) } 3 / 4 \text { frontal } \\
\text { 2) Profile (facing left) }\end{array}$ & $\begin{array}{l}\text { 1) Medium-high } \\
\text { 2) Medium }\end{array}$ \\
\hline $\begin{array}{l}\text { (7) BBC - Challenger } \\
\text { Tank }\end{array}$ & Medium & $\begin{array}{l}\text { Oblique ( } 2 / 3 \text { frontal } \\
\text { relative to tank } \\
\text { body/plane of motion) } \\
3 / 4 \text { frontal relative to } \\
\text { turret }\end{array}$ & Low (ground level) \\
\hline (8) BBC - Abrams Tank & Medium & 1) $3 / 4$ frontal & 1) Medium-high \\
\hline
\end{tabular}

(features matching the general pattern shown in bold, exceptions shown in italic)

The exceptions are frequently strategic in nature, such as for example using lower angles to emphasize the subject's power, or more oblique angles to create a greater social distance between the viewer and the subject.

Among images of aircraft, the pattern is also common although the use of the oblique and medium, rather than frontal and high angle appears with a particularly high frequency in depictions of fighter aircraft, for example as in the image below:

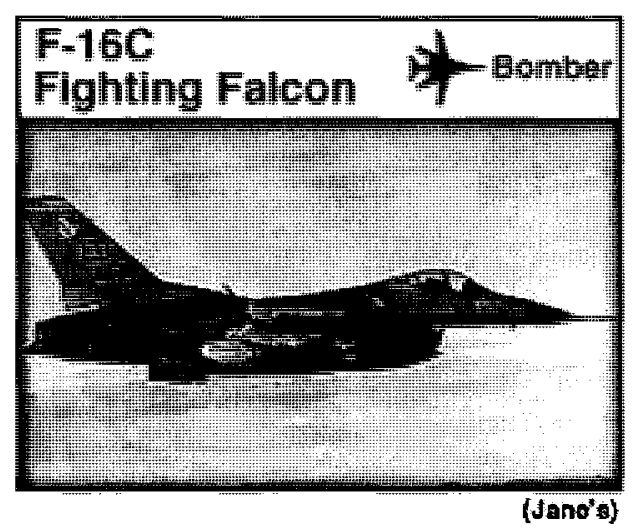

(CNN) 
This could be for a variety of reasons - it may simply be incidental to the appearance of fighter aircraft, which have a small frontal cross-section and short wings, but a very distinctive profile which has sharp, prominent diagonals - which lend themselves well to symbolising the speed and agility of the aircraft. This may also be done in order to make the aircraft appear less threatening to the viewer. After all, one reason that high angle is used in many of the pictures is to assign the viewer a position that is not only powerful, but relatively secure - underlining the fact that the ships are, perhaps, serving his/her cause.

Indeed, while it is possible to discuss perspective at length in abstract terms, one very basic and key question to consider is - how were these photographs obtained? Where was the camera positioned and in what relation was the photographer to the subject when the photograph was taken? It is easily possible to deduce the answers to these questions from the image's perspective. In the overwhelming majority of images of Coalition vehicles, the images were taken with obvious cooperation from the operators of the subject itself. Images of large objects like ships taken from a high angle were doubtlessly made from an aircraft flying overhead; images of flying aircraft from an airborne perspective - another aircraft flying in close formation. Airborne photography of military subjects is an expensive procedure which requires absolute cooperation with military commanders - and no doubt, a very privileged few are allowed to obtain this perspective, considering the security risks involved. And it is the obviously privileged nature of the perspective, more so than the specifics of vertical angle, that particularly empowers and aligns the viewer in relation to Coalition vehicles.

Another aspect of privileged perspectives used for publicity purposes, like airborne photography, is that they are markedly unusual and allow a much greater 
articulation of depth than ordinary photography would allow. In the majority of photographs in this section, depth is hyper-articulated beyond typical limits of naturalism. For example, consider the image below:

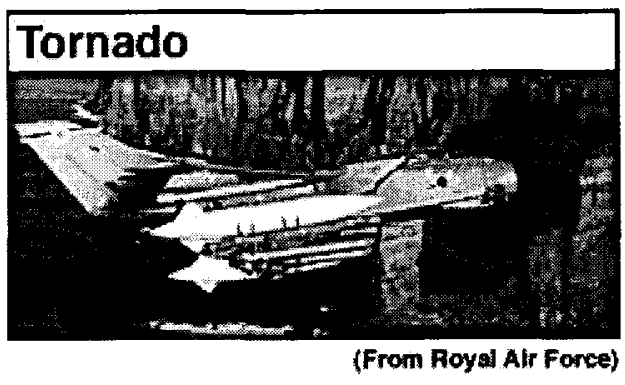

(11) $\mathrm{CNN}$

The sense of depth is firstly created by the juxtaposition of a foreground and background; secondly, the high angle is markedly unusual and was obviously achieved through airborne photography from another aircraft; thirdly, the aircraft is portrayed in a pose that actually exposes its underside and its weapons, which puts it in a highly dynamic position. Also of note is the notable use of highlights and shadows to give the aircraft's shape a sense of depth, the very narrow field of view produced by the lens which took this picture, the diagonals formed by the plane's outline, the dramatic use of lighting on the cliffs in the background, and the contrast/sharpness difference between foreground and background. While the somewhat detailed (if still unspecific) background is unusual for most images in this section, the rest of the pattern certainly extends to most portrayals of Coalition vehicles in articles by BBC (2003) and CNN (1998). Aside from depth the image also uses high brightness and colour variation to create a dramatic appearance. This type of image could not have been produced without elaborate posing, specialist equipment, access to Coalition resources - and also probably not without retouching software.

The effect of this marking is two-fold - firstly, as noted before, the viewer is placed in a privileged position relative to the subject; secondly, the viewer's attention 
is focused on the aesthetic attributes of the subject through an elaborate use of modality. The image thus exceeds limits of naturalism and moves into a sensory coding orientation, where this type of "hyper-realism" and certainly hyper-articulation of modality - especially depth - is expected.

As noted earlier, publicity photographs are intended not merely to present but to promote their subject. In this instance, they certainly accomplish the purpose firstly, by positioning the viewer at a social distance (through use of medium distance) but in engagement with the subject (through use of frontal angle); secondly by giving the viewer a privileged and secure position in relation to the subject (through use of high vertical angle and the literally privileged origin of the perspective in general); and finally, by providing a sensory-oriented, high-modality presentation of the subject that is aesthetically engaging.

The remaining element of the above images which has not yet been considered is the scale drawings that share the same space (and primary frame) with the photographic elements in BBC's primary illustrations. Stylized as technical drawings, the diagrams retain the focus on features and attributes of their subjects. As compared to photographs, they have extremely low modality on most counts - low contrast, no use of colour aside from shades of grey; a virtual lack of perspective articulation, except through overlapping lines and shapes; no background articulation and an absence of lighting in any form. The one modality that is relatively high is sharpness and detailed. The viewer is given access to what is presented as accurate, technical information on the dimensions and form of the subject, which is generally repeated to give multiple perspectives. There is no obstruction between the viewer and the details of the image, which certainly puts a viewer into a relatively powerful position but an abstract one, which by default creates a significant social distance. The main 
orientational purpose of the drawings is to present an unmarked portrayal to help support the highly modalized portrayal of the photo elements, thus grounding their sensory representation in more objective, technological reality.

\subsubsection{Summary}

Analysis of images of various Coalition vehicles included in the CNN (1998) and BBC (2003) documents on the weapons and forces of the U.S. war on Iraq reveals a number of consistent patterns and themes. Most of the visuals used in these articles are publicity photographs or combinations featuring them, and therefore largely sensory in orientation. This is manifested in the orientational aspects of the images, especially the heightened use of several modalities - including contrast, lighting, colour, and most notably depth of perspective, which are highly articulated. Representationally, the predominant pattern is of portrayals where single, inanimate participants - the vehicles themselves - are engaged in non-transactional actions (normally motion) or are simply presented in a way where attributes rather than roles are emphasized. There is a notable absence of human actors in most of the images. The perspective in most of the images is a privileged one, engaging the viewer while at the same time placing him/her at a distance - as evidenced by the predominance of moderate and moderate-long distances, high vertical angles and mostly frontal horizontal angles from which the images are taken relative to the main participant.

Since the overwhelming majority of the images of Coalition vehicles are used as primary illustrations, their role is to provide the general essence of the subject underscored by their positioning above the main text, which suggests their role as the ideal to the text's real. The essence presented is essentially that of inanimate, independent subjects engaged in vague, non-transactional actions - which results in 
the presentation being largely abstract and deemphasizes the connection of the subjects to material violence. By virtue of being placed into a privileged position and being afforded high-modality, aesthetically-attractive views of the military vehicles, the viewers are both aligned and drawn away from ethical concerns toward aesthetic ones by the authors of the discourse. Thus, the essence of the discourse presented in the images is indeed a highly idealized one - that of impressive, high-tech machines that are favourably disposed toward the viewer's interest. Representation of their involvement in material violence involving humans - either as actors or sufferers - is avoided as far as possible.

\subsubsection{Exceptions}

There are several minor but notable exceptions to the general pattern. Consider the images below, which have human participants and where the subjects seem to be placed in a much more circumstance-rich context:

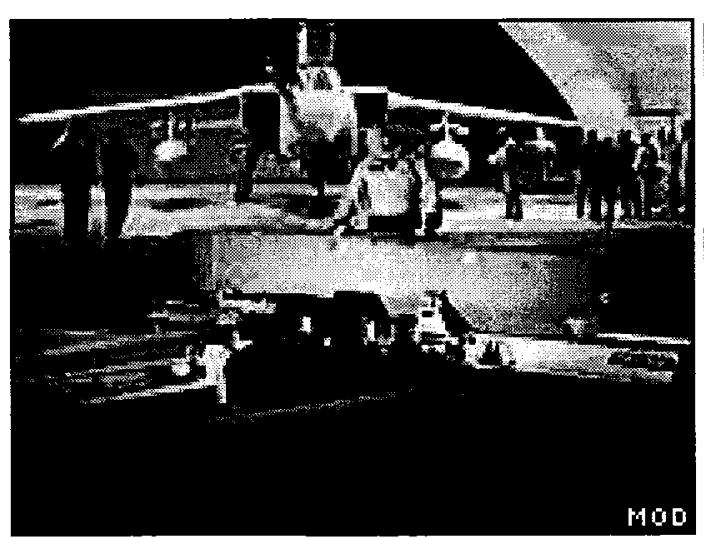

(BBC)

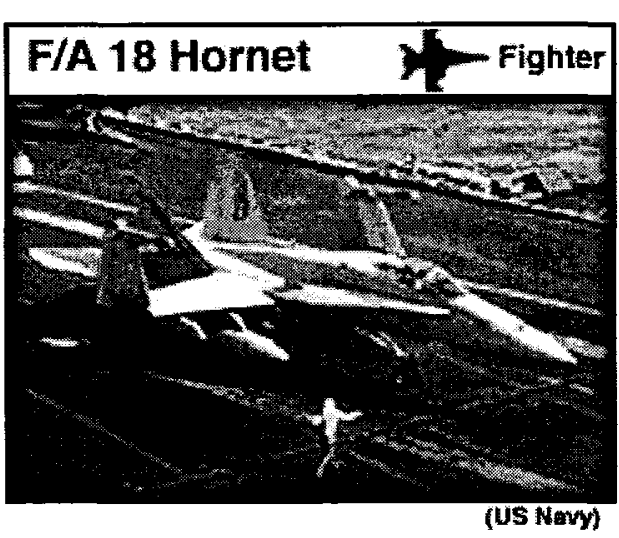

(CNN)

The first image is a secondary illustration appearing in a BBC article. In this image, the aircraft that is the centre of the article is seen in the background, with people surrounding it - this, in combination with the light, draws attention to it. In the foreground, there is also a weapon (a Storm Shadow cruise missile, as the subtitle to 
this image indicates) highlighted by a spotlight, and a man apparently examining it it is unclear whether he is doing anything to it materially, but his gaze is directed at the weapon.

This is an example of reactional process: though there are multiple participants and human actors are engaged, their role is a passive one - rather than simply showing qualities and attributes of the aircraft, the image shows people observing those qualities and attributes, thereby drawing attention to the aircraft and weapon at the centre of the discourse. This is further enhanced by the fact that it is impossible to see expressions of the human participants in detail - so the type of reaction is not specified and left up to the viewer (and other elements in the image) to determine. Perhaps a better way to classify them, then, is as a circumstance - that of both accompaniment, and possibly also that of means. The primary processes in the image are still relational - the aircraft and the weapon, for example, are implicitly related as parts of the same system, which is explicitly underlined by lighting - but the actual means for this process are the humans, which is perhaps their primary role in the image. The background is also more elaborated (if not completely specific) - it is readily recognizable as an aviation hangar at an allied air base.

In the second image, the interaction is far more overt however - one can interpret the process taking place between the person and the aircraft as a reaction or a verbal process. The person in the image is either reacting to the movement by the aircraft and stepping out of the way, or giving signals to the aircraft's pilot (or perhaps both). Either way, the aircraft remains dominant within the image. The background in this image, like the previous one, is also more elaborated - it is relatively easy to distinguish the setting as an aircraft carrier deck, which brings up its own chain of association. 
The effect of inclusion of human participants and more elaborate background is the production of narrative - one that is centered on the main subject, aircraft in the case of the images above. The text accompanying the images reinforces this - "New weapons - including the Storm Shadow cruise missile - and upgrades now mean that the Tornado is more versatile" for the first image, and "The F/A-18 is a single-seat carrier-based aircraft, flown by both the Marine Corps and the Navy" for the second. The narratives are thus about upgrades for the airplane in the first case, and about carrier operations in the second - relatively complex processes represented quite effectively with simple depictions. Humans help mediate these narratives, give a sense of scale and direct attention towards the aircraft (or in the first case, aircraft and weapon).

Orientationally, these images are not remarkably different from other images found in the section. While the modality is not extremely high on either, both still use perspective to significant effect, placing the viewer in a privileged position relative to the aircraft (and weapon). Lighting and contrast are sharp in both images, though play a more significant role in the first. The aircraft are presented clearly and with numerous attributes visible, which cannot be said of the humans and background. Organizationally, it is clear that the aircraft is the centre of the discourse, with humans placed very much in the peripheral position. Thus, as with all preceding examples, the primary role of the image is presenting attributes of the aircraft and aligning the viewer in relation to it. The simple narratives in these exceptional cases only serve to underline this purpose. 


\subsection{Section II - Coalition Troops}

The second section of analyzed data is comprised of images found in articles related directly to the description of coalition troops and military units - including formations of the US Army and Marines, their British and Australian equivalents, and also cooperative Kurdish militias working against the Iraqi regime.

Unlike the preceding section where there was a set of predominant patterns extending to the majority of the images and an approximate equivalency between $\mathrm{CNN}$ and $\mathrm{BBC}$ in terms of types of images represented, this section presents some organizational difficulties: firstly because there is only a single illustration dealing with troops in CNN's (1998) documents; and secondly because within BBC's (2003) Fact Files, there is a variety of images that do not fit into a single pattern. In the case of $\mathrm{CNN}$, the lack of images relating to Coalition troops is due to its focus on presenting the 1998 air and sea campaign, where the use of troops on the ground was not a very likely possibility. The variety in BBC's images, meanwhile, is caused primarily by the complex and diverse content that they relate to. The overall distribution of images is outlined in the table below:

Section II - Coalition Troops

\begin{tabular}{|c|l|l|l|l|}
\hline & Total & $\begin{array}{l}\text { Primary } \\
\text { Illustration }\end{array}$ & $\begin{array}{l}\text { Secondary } \\
\text { Illustration }\end{array}$ & $\begin{array}{l}\text { Exposition/ } \\
\text { Explanation }\end{array}$ \\
\hline CNN & $\mathbf{1}$ & $\mathbf{1}$ & & \\
\hline$\bullet \begin{array}{l}\text { Ordinary } \\
\text { Photograph }\end{array}$ & $\mathbf{1}$ & 1 & & \\
\hline BBC & $\mathbf{3 5}$ & $\mathbf{2}$ & $\mathbf{2 9}$ & \\
\hline$\bullet \begin{array}{l}\text { Ordinary } \\
\text { Photograph }\end{array}$ & $\mathbf{1 0}$ & & 10 & \\
\hline $\begin{array}{l}\text { Publicity } \\
\text { Photograph }\end{array}$ & $\mathbf{2 1}$ & 2 & & $\mathrm{I}$ \\
\hline $\begin{array}{l}\text { Scale Drawing } \\
\text { / Diagram }\end{array}$ & $\mathbf{1}$ & & & 3 \\
\hline $\begin{array}{l}\text { Function } \\
\text { Diagram }\end{array}$ & $\mathbf{3}$ & & & \\
\hline
\end{tabular}


The distribution does show a predominance of photographic imagery used in a secondary illustration role within BBC's articles. The analysis will thus focus on examining this photographic imagery. Scale drawings and function diagrams will be considered in detail in Section IV, where these images predominate.

The sample analysis below will examine a selection of images individually in order to establish patterns and strategies built into them. While unfortunately not comprehensive, this examination should provide a look at the representation of this complex and difficult subject - since, unlike presentations of machinery, it has to deal with direct involvement of humans in conflict. Like some of the exceptional cases to the portrayal of Coalition vehicle discussed above, images of troops frequently rely on abstract personalization and use simple, relatively abstract narratives that work in conjunction with the text. These, along with other strategies, help align the viewer but also keep him/her at a social distance if necessary.

\subsubsection{Image 1}

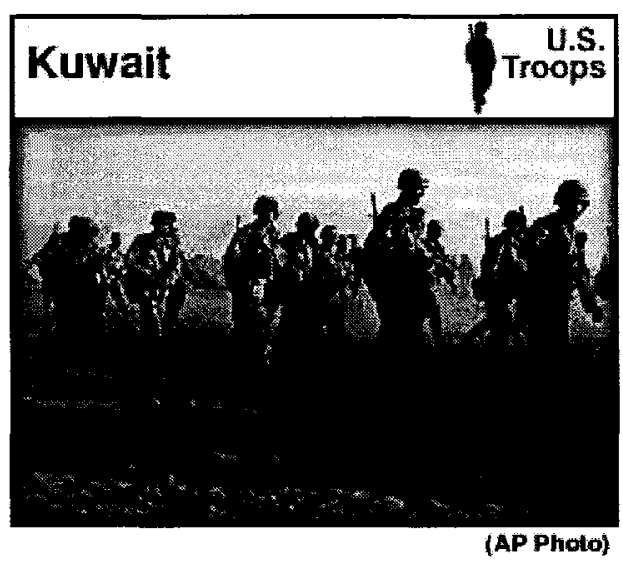

(1) $\mathrm{CNN}$

The sole image representing Coalition troops within CNN's (1998) special feature on the weapons and forces of the Iraq conflict is located in a very short article describing the U.S. forces stationed in Kuwait, and ready to deploy if it becomes necessary as a 
result of the 1998 air campaign. Their role in the situation is implied to be minor at best - if only by the brevity of the accompanying text, which is comprised of a single sentence: "The United States maintains a significant military presence in Saudi Arabia to patrol the southern "no-fly zone" over Iraq, including some 6,000 ground troops." Formatted similarly to primary illustrations discussed in the previous section, the image is comprised of two frames: a smaller top frame containing a title on the left and a silhouette with a subtitle on the right, and a prominent bottom frame containing the photograph itself surrounded by a black inward gradient border. The photograph is a primary illustration, and is thus positioned at the centre and top of the text it accompanies. By virtue of its vertical positioning, the image serves as the Ideal to the text below, summarizing the essence of the subject in idealized, general terms.

The most salient element in the photograph is the figures of the troops, emphasized through contrast and lighting - which makes them stand out significantly from the background, a central position vertically, as well as repetition. They are identified by attributes including camouflage clothing, helmets and guns; in addition, the silhouette and its subtitle in the top frame help ensure that the viewer understands these to be US troops.

The troops are also the only participants in the image, engaged in a nontransactional material process of walking. The circumstances are relatively vague, but the desert setting in combination with the title suggests that the troops are pictured in Kuwait. The lighting in the image suggests that the scene takes place at dawn or dusk, though this is not of any apparent importance. The repetition helps emphasize the direction of movement, and the dust seen in the background also symbolically suggests a large number of men to be on the move - but without a specified goal, the 
narrative structure in the image remains abstract and thus can be incorporated into virtually any discourse about US troops in the Middle East.

Once again, the more predominant processes and structures in the image are of the relational type - emphasizing group identity foremost. Firstly, the image establishes a covert taxonomy by placing participants in parallel, meaning the soldiers are presented as a group of equivalent participants. Secondly, the image avoids directly personalizing any of the troops or assigning them attributes that distinguish them from each other. This makes it relatively easy to construe the image as a portrayal of any given group of soldiers present on the ground in Kuwait.

Orientationally, the image is taken from a moderately long distance from its subjects, from a medium vertical angle (approximately eye level), with an oblique angle (approximately $2 / 3$ frontal) towards its participants. Some of the soldiers are facing towards the camera, however the detail is obscured by shadows from their helmets, and further reduced by the distance. All of this serves to place a social distance between the viewer and the subjects in the image; the oblique angle, in particular, is typically associated with specifying that the viewer is not engaged in the same field as the participants in the image. At the same time, the viewer's position, by virtue of its clarity and a high articulation of depth, can be considered a relatively privileged one, if not as much as ones observed in the previous section. There is certainly some measure of engagement and cooperation from the troops observed. The photographer is certainly aligned and possibly affiliated with the troops, which is even expressed in the fact that he/she is allowed to approach this closed to an armed unit on patrol.

The image has a high articulation of several modalities, most prominently contrast and lighting, which are used to both highlight participants and obscure certain 
details of them, and to create a dramatic atmosphere. While the image may not be as highly marked as some discussed previously, it is still primarily sensory in orientation.

Thus, the image is focused on the figures of troops and a limited number of their attributes - while others, including facial features, are largely obscured. The soldiers are involved in a non-transactional action (walking) only, with circumstances articulated only to a minimal extent. The viewer is in a relatively privileged position in relation to the troops, but the medium-long distance and oblique angle still serve to place the viewer at a social distance and to disengage him/her from their realm of experience. This depiction distances the viewer, aligns him/her with the troops to a certain extent, and avoids explicit reference to material violence.

\subsubsection{Image 2}

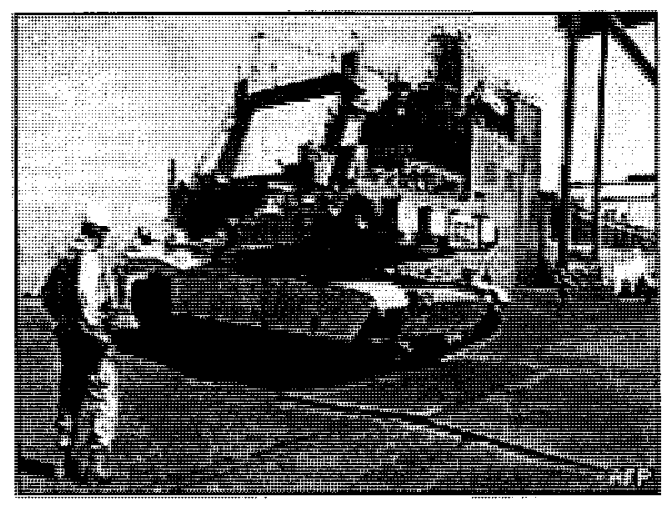

(2) BBC

The image above is a secondary illustration accompanying a $\mathrm{BBC}$ article describing the US $4^{\text {th }}$ Infantry Division. As the article accompanied by this photograph notes, the division is equipped with a large amount of high-tech equipment like advanced tanks - and even the subtitle directly below the image indicates that "Abrams tanks are one of the division's main armaments". This division was intended to be used on the frontline in Iraq, but political complications over its deployment to Turkey for the 
invasion of Iraq resulted in the division spending the initial part of the campaign being re-deployed by sea to Kuwait (BBC, 2003). The image picks up these narratives effectively and gives the viewers a positive association for these features of the unit's operation.

Being a secondary illustration, the image is in a very different relationship with the text than the images discussed previously. Rather than setting the theme for the article and working in an ideal-real relationship relative to it, the illustration is instead dependent on the article, drawing on aspects mentioned within the text - and, being positioned to the right of the article, serving as the new to the article's given. The article's role is thus not to present a distilled essence of the subject, but expand on various features of it mentioned in the text. To an extent, this frees the creators of this media from having to make the complex choice of how to distill this essence and also affords them a greater choice of narratives and features to depict. This is the primary factor accounting for the variety of secondary sections seen in this section.

The image does serve as the ideal to the subtitle, which specifies the type of tank depicted as the most salient element in the image, and notes its importance to the division. There is a clear foreground-main subject- background structure within the image, with a man taking a prominent position in the foreground, the tank serving as the central subject, and harbour structures as well as a ship in the background. The tank is both physically in the centre of the image, and also in the centre by means of depth created in the image, thus orienting the image around itself. The levels of distance and the clever orientation of the participants suggest a temporal symbolism, moving from foreground to background - indicating where the tank is headed. The tank is thus implied to be in a material process of motion toward the ship; it has already passed the harbour worker at foreground, who was perhaps involved in this 
process but is now standing back and observing the tank, thus engaged in a reactional process. Since the type of reaction is not specified, this serves primarily as a device to draw attention to the tank. Alternatively, the worker could also be classed as a circumstance - explicitly of accompaniment, and if one reads further into his role in the narrative, perhaps also that of means. Thus, a relatively simple and abstract narrative is established without assigning a specific reaction or personalization to the human participant in the image. The narrative conveniently connects to the larger narrative established in the text.

Orientationally, the viewer is positioned at medium-long distance from the tank and the dock worker; an oblique horizontal angle is used, with all participants shown primarily from the back, but facing in different directions. This serves to create social distance and disengage the viewer from the immediate context of the illustration. The image is taken from an ordinary (i.e. not particularly privileged) perspective, at eye level, without apparent engagement from the participants - thus it cannot be said to be highly privileged, although it does provide an unusually clear view of the subject and its context. The photograph is, in fact, very carefully composed, which becomes apparent through the very efficient and highly marked articulation of depth using a very wide-angle lens, positioning of subjects, diagonals, and lighting. The image retains high sharpness and contrast, and participants are presented in high detail, given the distance. Though shades of grey are very predominant, there are also items that use a high saturation, such as the sky, as well as the colours of the worker's clothes and the tank. Thus, while remaining within limits of naturalistic modality, the image certainly articulates them to a significant extent to draw the viewer's attention to the aesthetic dimensions of the image. 
Thus, the image is organized in a way which creates a simple narrative which works well in reference to a passage in the accompanying text, helping to expand on it - which is underlined by the fact that the text is on the left of the page, with the image on the right, thus working in a given-new relationship. The viewer is positioned to have a privileged but disengaged view of the narrative. The role of the human participant is primarily a reactional or circumstantial one, with the primary emphasis still being placed on the role of machinery. Furthermore, the narrative is about an aspect of the unit which has no direct relation to material violence. Thus, the image once again implies ideological alignment and distancing.

\subsubsection{Images 3 and 4}

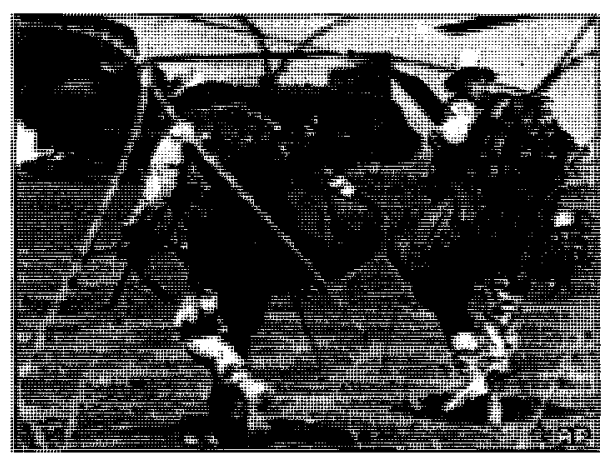

(3) $\mathrm{BBC}$

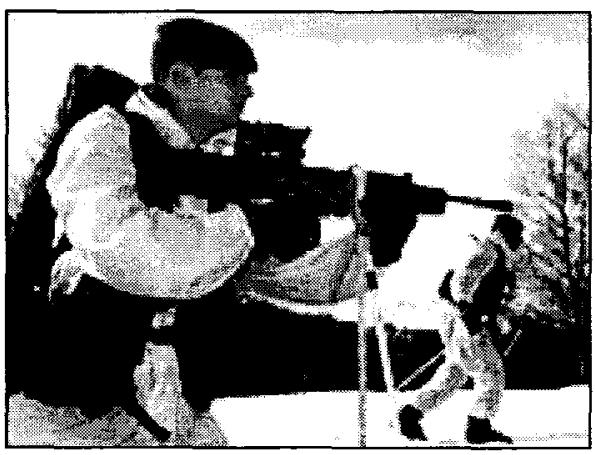

(4) BBC

The two images above are secondary illustrations appearing in the BBC articles about the US $101^{\text {st }}$ Airborne Division and the British 3 Commando Royal Marines respectively. The two photographs share a number of key features and present similar themes, while also presenting orientational differences which are useful to compare and contrast, and therefore are analyzed together in this section. The first image shows two armed soldiers with a heavy load of equipment walking towards a helicopter; they are physically separated from the photographer by a barbed wire fence. The subtitle underneath the image notes the division's participation in the 1991 Gulf War. The second image shows two soldiers on skis, with the subtitle suggesting 
that the location is a training ground in Norway where the division has previously trained. Thus, the illustrations show narratives of typical situations in which the units may be involved, and also connects these to specific operational history via the subtitles.

The soldiers are the most salient elements in the images, by virtue of contrast, position and repetition. In the image on the left, the soldier closer to the viewer is predominant, with the gun being the most recognizable of his attributes. An unusual aspect, given the images examined so far, is that his face is visible relatively clearly. For depictions of soldiers, this is actually fairly common in the data as a whole. BBC's (2003) photographs do portray facial expressions and a form of personalization where it serves the overall effect of the image. The personalization created here is abstract - but it is possible to see the soldier's expression, which can be characterized as relatively serious, and the direction of his gaze to the right of the frame, to an unseen object somewhere outside of the viewer's sight - which suggests that he is engaged in a reactional or mental process, with the object of this process unspecified. The gun, being aimed in the same direction, serves as a circumstance of accompaniment, or perhaps means - if we interpret the process as aiming, rather than simply looking. The soldier in the background of this image, meanwhile, is seen running on skis in the same direction, serving as accompaniment and symbolically helping emphasize cooperation between troops. The setting is obviously daytime in a winter landscape, including snow, trees without leaves, and distant hills in the background. The subtitle indicates that the setting is Norway, and it is relatively easy for most viewers to associate this northern country with winter and hilly landscapes.

The image on the left, by contrast, uses what appears to be a desert landscape and alludes to the Gulf War, onto which the image could certainly be projected. 
Unlike the second image, while the soldiers' faces are not seen, their gaze and the direction of their movement appear to have a goal - the helicopter in the background. In particular, the open door of the helicopter is emphasized through clever use of internal framing - its frame formed by the outlines of the two soldiers to the sides and below it, and the helicopter's rotor above. The clear juxtaposition of foreground, center and background with movement directed outward suggests a temporal structure - indicating where the soldiers will be in subsequent moments. Once again, the image presents a short, relatively unspecific narrative.

Orientationally, the two images are quite different. In the first image, there is a significant social distance and sense of detachment, created firstly by the physical barrier placed between the viewer and the soldiers by the barbed wire; secondly by the facing of the participants away from the viewer, a horizontal perspective from the rear, and a complete lack of apparent engagement with the viewer. This, of course, conflicts with the relatively close distance, and high articulation of depth, as well as very careful positioning of the photographer - so this distance is largely artificial and deliberate. Perhaps this is motivated by the need to fit the image to the subtitle which alludes to the 1991 Gulf War, stating that "The 101st Airborne saw action during Desert Storm" (BBC, 2003) - thus helping create a temporal distance, distinguish the previous war from the upcoming one, but also of course emphasize the secure position of the viewer. As Kress and van Leeuwen (2006) note, the back perspective can suggest a measure of trust - since on the one hand exposing one's back can be a risk, and on the other hand being placed directly behind a person, suggests protection and safety (p.138). The image is perhaps intended to show soldiers going beyond the viewer's realm of experience, for the sake of the viewer's security - thus building a relation that is markedly distant but favourable to the viewer. 
Given the careful positioning of the photograph and the marked use of depth, focus distance, contrast, lighting and well-defined colour, perhaps the viewer is not in such an unprivileged position as the image may initially suggest.

The second photograph, meanwhile, shortens the social distance between the viewer and the photo's subject through relatively close distance and clarity of facial features, but also keeps the viewer disengaged through the oblique angle and lack of facing or eye contact. Of particular importance in this image is the vertical image, which is moderately high, placing the soldier in a powerful position above the viewer. This, combined with the serious expression and determined posture, as well as relatively extreme circumstances (fighting in the snow is at least somewhat unusual), emphasizes the soldier's strength, ability and determination, essentially suggesting that the viewer trust them to act - even if the viewer cannot see what it is they are acting upon. The close distance helps keep the viewer aligned with the soldiers. Otherwise the image articulates depth very highly through multiple levels and use of focus; the posing is quite deliberate; and contrast, lighting and sharpness are used to highlight the participants.

Thus, while the images above focus on the human roles in the conflict, reducing the social distance and placing the viewer in a more direct relation with the troops - they also disengage the viewer from the soldiers' realm of experience and once again shift focus to attributes rather than actions. The narratives produced by the images highlight aspects of the units' history and contextual use, but they avoid any representation or overt reference to material violence. The markedly high use of modality also helps direct the viewer's attention to aesthetic concerns and away from ethical ones. 


\subsubsection{Image 5}

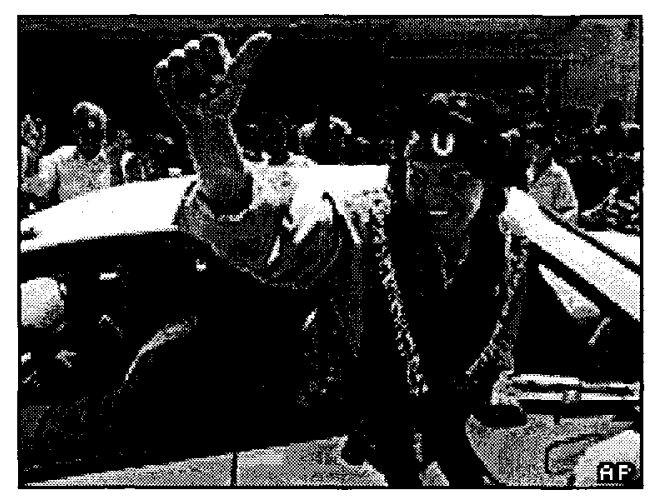

(5) BBC

The image above accompanies the BBC article about Kurdish militias in Iraq, such as the PUK, who are friendly to Coalition forces and opposed to Saddam Hussein's regime. This illustration demonstrates one particular aspect of these militias, denoted by the image's subtitle: "The PUK peshmergas include a women's battalion" (BBC, 2003). The image shows a street scene, with a car driving by, carrying a saluting female combatant, surrounded by what look to be cheering civilians.

The image is a secondary illustration, placed on the right of the article's main body, suggesting that it is in a Given-New relationship with the text, serving as the New. Within the text, the existence of this female battalion is only mentioned in passing - "The vast majority are men, though there is a 500-strong PUK women's battalion, run by 27-year-old Lieutenant Sirwa Ismael, which was formed in 1996" (BBC, 2003). This provides a short, specific reference, but the image helps expand on this topic and to fill the interpersonal details, and is thus particularly rich in orientational meanings. Without the image, the theme of female combatants, while marked, would have been relatively understated in the context, however with the image it gains much for the article in terms of response from the viewer.

The most salient entity and primary participant is obviously the female fighter. Perhaps the main reason this image had been chosen is because of how explicitly it 
denotes the allegiance and function of this person - the recognition of the person as a woman may be tacit, but items such as the prominent assault rifle and the headband which bears the letters "PU" (presumably part of "PUK") indicate in no uncertain terms what the woman's role is.

The primary processes taking place in the image are reactional ones, although the car is implied to be moving, with its driver seemingly concentrated on this task and unlike the other participants is uninvolved in the interaction and positive reactions. The object of the woman's reaction is unclear - the reaction is not directed at the viewer, and the occasion for this image is not specified. The reaction, however, is left in no uncertain terms - the facial expression and gestures she makes indicate jubilation and approval. The sort of posturing in which she is engaged, her military role, and the surrounding crowds do suggest that she is perhaps celebrating victory. The civilian bystanders appear to be reacting to the woman in a very positive fashion. The old man and children on the left side of the frame appear to be smiling and waving in her direction. The circumstances, with sand-colored walls and market stalls indicate the setting to be somewhere in a Middle Eastern city, though there are no further specifics given.

Relative to the woman in the centre, the image uses a slightly offset frontal perspective, which draws the viewer into the reactions, and also emphasizes depth of perspective. At medium-close distance, the subject is placed in relatively close social proximity to the viewer. With the gaze of the fighter directed away from the camera, the image avoids making a demand on the viewer, however the frontal facing and prominent, articulated facial expression do invite the viewer to engage in the reactions of the fighter and the civilians. The vertical position of the camera is slightly above the level of the participants, which along with proximity and highly articulated 
perspective, places the viewer in a relatively privileged position that aims to directly place him/her in a positive relationship with the participants in the image.

This image is the most affectively engaging out of those found in the data, which is accomplished through marked and recurrent use of facial expressions and reactional processes, predominance of human participants, use of frontal and moderately high angle, along with relatively close distance to the primary participant. This is a powerful alignment strategy, and used in this case to create sympathy for subjects that are distant from most Western readers' experience and familiarity. Group identities and types play a key role here - due to the obvious civilian presence and the very positive nature of the reactions taking place, the position of the rebel group and especially their female battalion is legitimized and given positive connotations, as representative of the population's will and sympathies. At the same time, any association with material violence in this image is at best distant and indirect - with only the gun in the fighter's hand making any reference to it.

\subsubsection{Image 6}

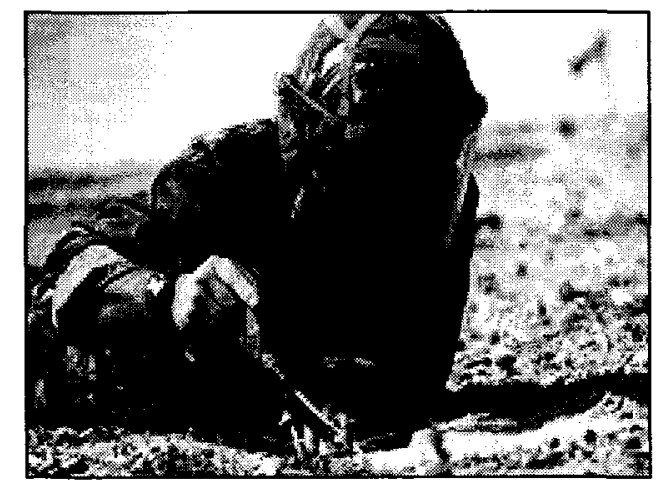

(6) $\mathrm{BBC}$ 
The image above is used as a secondary illustration in the BBC "Fact File" on the British 33 Engineer Regiment. As such, it serves to display one aspect of the unit's operations described in the accompanying text - mine disposal.

The most salient entity in the image is the soldier, mainly through high contrast and sharpness against the background. The viewer's attention is directed by a prominent vector formed by the pointed tool in the soldier's right hand - presumably, toward an unseen mine with which the soldier is working. Thus, there is a presumably transactional material process taking place, mediated by the relatively nondescript tool which serves as the means for the action. With the object invisible, however, the attention of the viewer turns toward the subject and his/her attributes, including the large face shield worn for protection. The circumstances are blurry but symbolic - the leaning sign or flag in the background, supporting the activity in which the soldier is involved, suggest this relatively unremarkable area of grass and dirt to be a mine field.

The perspective places the viewer at a relatively close social distance to the soldier, but at the same time keeps a reasonable physical distance - which is accomplished through using a very narrow-angle lens and using focus length to produce significant depth, which is hyper-articulated for dramatic effect, with the sharp participant against a blurred background suggesting tension. The soldier's facial features are understated and the eyes are not seen at all - although it is presumed that his/her gaze is directed toward the ground.

The image articulates contrast, sharpness and saturation to a very high degree, exceeding naturalistic modality to give an aesthetic portrayal of the tension involved in this process. Presenting a clear (yet unspecific) view of this process, it places the viewer in a privileged (if somewhat insecure) position. Certainly, the viewer would be aligned with the soldier in the photo, with the social proximity ensuring sympathy. 
There is no doubt the photo itself is staged and carefully articulated to suit this purpose, which is primarily sensory as it gives few specifics of the process but uses sharply-articulated depth, contrast and colour to engage the viewer's attention and emphasize the atmosphere of the image. The image is likewise focused less on personalization, which despite the close distance is subdued, but rather the inherent tension.

\subsubsection{Image 7}

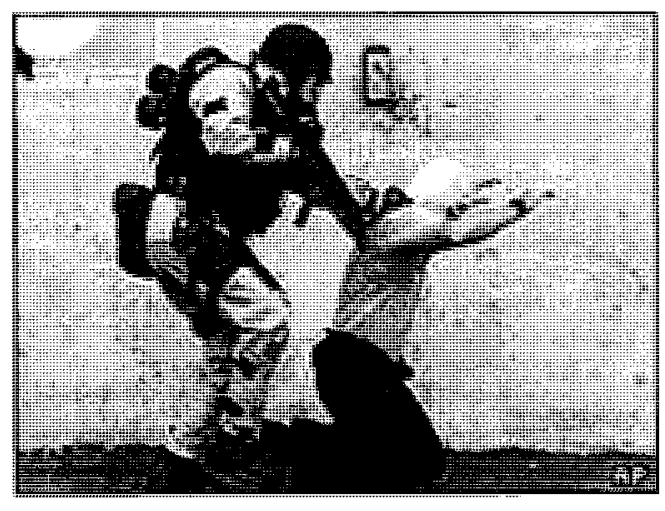

(7) BBC

This image is a secondary illustration found in the $\mathrm{BBC}$ article about Australian

Special Forces. The salient property of this image is that of the 31 photographs of Coalition troops contained in the BBC (2003) Fact Files, it is the only one containing a directly transactional process.

This transactional process takes place between two participants - a Special Forces soldier, who is easily identified by his attributes, such as weapons and protective gear, and a man in blue jeans and grey shirt, with what appears to be a bag or mask over his head. There are two aspects to the process itself - firstly, there is a purely material action suggested, with the soldier physically subduing the man, as suggested by the placement of the gun and the hand placed on the subject's back, both 
of which serve as the means; secondly, one can also interpret the action as a verbal process and the reaction to it - certainly, the raised hands indicate that the man in the image is reacting with active cooperation. The poses in the image are highly dynamic - showing the participants in mid-action, thus it is certainly difficult to exclude a strong material element to the processes taking place. The circumstances of setting are not particularly specific - a grey concrete wall with yellow signs and markings on it, and a dark grey pavement on the ground underneath. One can assume the action to be taking place in a modern urban environment.

The subtitle beneath the image, serving as the real to the image's ideal, is more crucial to placing the image than the visible setting: "Counter-terrorism: Expanded role at home" (BBC, 2003). Thus, it is understood that the action actually takes place in Australia, with roles different than ones expected in 1raq. The illustration's placement to the right of the article, which notes the recent formation of this counterterrorist role, further emphasizes its role as the new (to the article's given). It is, of course, peculiar that the only image in the entire section showing transactional material action is specifically marked as taking place outside Iraq - 'at home'. This is both telling of the distancing and abstraction tendencies of the other images in the section which avoid transactional actions; and of the highly marked nature of any direct portrayal of violence.

Meanwhile the oblique horizontal angle and medium distance disengage the viewer from the action taking place, thus placing him/her in a position that is at least safe, and at most - privileged. While the latter is not expressed to a significant extent through perspective in this case, there is certainly high use of modalities, particularly colour and saturation, contrast, sharpness and depth (through lighting and pose) to engage the viewer's attention. 
That high modality and the highly unusual nature of the photo may cause the viewer to wonder whether the photo is staged, or at least part of a training exercise. While it is difficult to give a completely certain answer to this without information on the origin of the photo, this is at least plausible and likely. And even if it does show real violence, one must admit that the portrayal is at least quite subdued, carefully composed, and not without aesthetic elements. Despite being an exception to the representational pattern by portraying material violence, the image still does not fall as far outside the orientational and organizational norms seen thus far. A high degree of social distance and disengagement from the action on the part of the viewer is maintained, and certainly the broader ideological goals of abstraction and distancing are maintained as far as possible.

\subsubsection{Summary}

The seven images examined above reveal a number of different ways in which the subject of Coalition troops is approached visually, as well as a substantial number of similarities between photographs used in the section.

Firstly, the predominance of publicity photographs points to the primarily sensory, and thus highly modalized and aesthetically-driven nature of the images. Although not with the same consistency as in the case of Coalition vehicles, the viewer is still frequently placed in privileged positions relative to coalition troops.

Secondly, much like the exceptions discussed in the first section, the photographs seen here present simple, if often vague narratives which focus more on attributes and circumstances than material actions. With a single exception, emphasis on non-transactional actions and avoidance of direct portrayals of material violence are maintained. 
Otherwise there is a greater variety of subjects and methods of representation in this section than in previous section - and indeed in all sections of the data. Part of this is due to the unusual predominance of secondary illustrations, which place less pressure on the authors to capture an idealized essence of a subject, instead showing individual features and aspects of the subjects presented in the articles. These illustrations thus present more strategic flexibility - and thus sometimes allow for contrasting choices. Where the soldiers need to be portrayed strong and determined, they might be shown from a low angle - as seen, for instance in image 4; elsewhere the subjects are presented from medium or even relatively high angles. Where social distance needs to be broken down and affective reactions are desirable (for the purposes of alignment), the participants are placed in relative proximity to the viewer, and facial expressions are prominent and articulated - such as seen in image 5 . Where an obvious separation is needed between the viewer and the subjects of the photo visual barriers, longer distance and lack of facing towards the viewer are used, such as in image 3. It is even possible to display direct transactional action - even violence between two participants, as seen in the final image - as long as it is marked and clarified by the subtitle.

Therein lies another notable aspect - the secondary illustrations seen on BBC draw on the text to a significant extent to guide the viewers' interpretation of the images. Whereas in the previous section the images remained in a more predominant role - setting themes and ideal representations for the text, the illustrations of Coalition troops expand on themes that had already been set in the text. In other words, there is a greater variety of images and a greater focus on simple narratives because secondary illustrations fit into the text on a much narrower level, or lower rank, than primary illustrations. This allows them to be qualified more thoroughly 
before the viewer even gets to them. Thus, while the overall patterns of alignment, distancing and abstraction is retained, there is a greater variety of ways in which it is expressed, and not all images fit in with the most expected ways of accomplishing these goals.

\subsection{Section III - Iraqi Forces and Equipment}

Perhaps the first ideological choice in the visual portrayal of Iraqi forces in the CNN (1998) and BBC (2003) documents lies in the lesser coverage given to them - with only 18 images of 177 in the total sample being dedicated to them. It seems that on the whole, the role of the Iraqi forces in the war is de-emphasized, and their depictions are not given the same importance as those of Coalition weapons. A summary of the overall distribution of these images is found below:

Section III - Iraqi Forces and Equipment

\begin{tabular}{|c|c|c|c|c|}
\hline & Total & $\begin{array}{l}\text { Primary } \\
\text { Illustration }\end{array}$ & $\begin{array}{l}\text { Secondary } \\
\text { Illustration }\end{array}$ & $\begin{array}{l}\text { Exposition / } \\
\text { Explanation }\end{array}$ \\
\hline CNN & 6 & 4 & 1 & 1 \\
\hline $\begin{array}{ll}\text { Ordinary } \\
\text { Photograph }\end{array}$ & 3 & 3 & & \\
\hline $\begin{array}{l}\text { Publicity } \\
\text { Photograph }\end{array}$ & 2 & 1 & 1 & \\
\hline $\begin{array}{ll}\text { - } & \text { Function } \\
& \text { Diagram }\end{array}$ & 1 & & & 1 \\
\hline BBC & 12 & 4 & 7 & 1 \\
\hline $\begin{array}{ll}- & \text { Ordinary } \\
& \text { Photograph }\end{array}$ & 6 & & 6 & \\
\hline $\begin{array}{ll}\text { - } & \text { Function } \\
& \text { Diagram } \\
\end{array}$ & 1 & & 1 & \\
\hline - Combination & 5 & 4 & & 1 \\
\hline
\end{tabular}

This distribution certainly suggests some variety in the patterns observed in this section. Firstly, there is an obvious difference in the strategies used by $\mathrm{BBC}$ and $\mathrm{CNN}$, with $\mathrm{BBC}$ once again fielding a greater variety of images of each type. Secondly, one can certainly see some parallels to the portrayals of Coalition vehicles and troops discussed earlier. Finally, it would be expected that there would be variation based on 
the subjects of the images themselves, with troops and equipment each having their own properties.

The analysis in this section suggests that the Iraqi forces are portrayed in subtly, but importantly different ways from Coalition troops. There is a greater predominance of ordinary photographs, which have a lower articulation of the same modalities which are generally articulated highly in equivalent images pertaining to the Coalition. This is part of a wider pattern of placing the viewer into unprivileged positions relative to Iraqi troops and combat vehicles. There is also a notable pattern of distancing and disengagement through a number of the images, using a range of devices including perspective, framing and obstruction of the viewer's line of sight. Where this distancing is not observed, however, another pattern appears to come into play - the use of low frontal angle to place the viewer in a vulnerable, implicitly threatened position relative to the Iraqi soldiers or equipment, which invites more negative reactions. And while the discussion of text patterns lies outside the scope of this study, it is worth noting that the content and format of the articles accompanying the images is significantly different from anything observed in regard to Coalition forces before - with more thorough (and thoroughly critical) analysis of Iraqi capabilities which moderates this implied threat to a significant extent, suggesting that while these soldiers and weapons may present a danger, this would be a marked exception rather than a norm for their performance in any actual war.

The analysis below will consider portrayals of troops first, and discuss the images showing Iraqi vehicles second, further below.

\subsubsection{Troops}

Both BBC (2003) and CNN (1998) present a total of two articles each on the topic of Iraqi troops, dividing them into the regular Iraqi Army, forces under the command of 
the Iraqi Defense Council and Ministry of Defence, and the Republican Guard - an elite force under Saddam Hussein's direct command (Jane's, quoted in CNN). Below, the portrayals of both in the CNN (1998) documents can be seen.

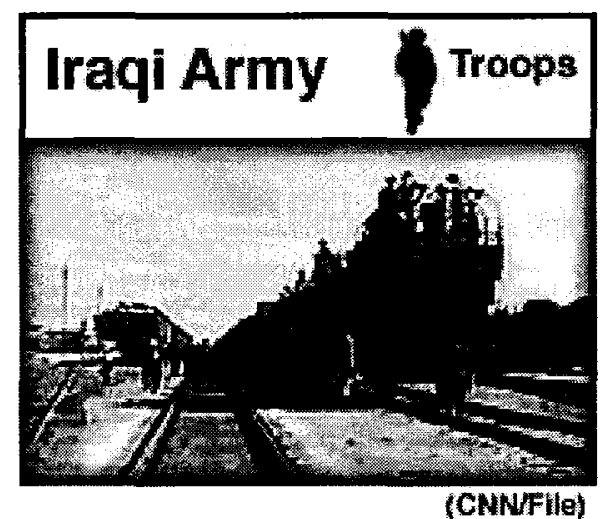

(1) $\mathrm{CNN}$

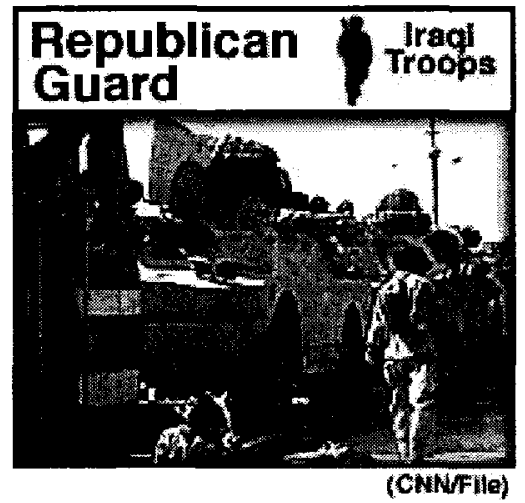

(3) $\mathrm{CNN}$

Both images are primary illustrations, used at the top of their respective articles. They thus form the ideal representation to the article's real, suggesting that their contents are the general essence of the subject. Both of the images are composed of two frames - a smaller frame with the title and silhouette of a soldier on the top, and a larger frame surrounded by a black gradient border containing the photograph itself on the bottom. The two frames are also in an ideal-real relationship with each other - and one could also note that the silhouette of the soldier is common between the two images, as well as the only image of US troops discussed in the previous section.

The first image shows what appears to be a train station, with the nearest train having multiple human silhouettes on top of it. Other human silhouettes surround the train. The details of the station are not particularly clear - one can see other trains, rails, sand or dirt on the ground, and what appear to be electrical power lines and buildings far in the background on the left side of the image.

The rail lines and train cars, along with the strongly-defined convergence point to the centre-left of the image, form a strong vector leading away from the viewer. Although the human figures are distant and unclear, it appears that most of those on 
the train are facing away from the viewer, and there are no distinguishing attributes visible on them. Thus, one can only infer any sort of narrative here with the help of the title and context - perhaps these are troops being carried away by a train, which is a relatively low-tech transportation means, especially considering that the train cars which are shown appear to be hardly specialized for carrying people.

Orientationally, the most notable properties of this image include a distant, low perspective - with the image being captured from approximately eye level by someone crossing the road. There is no engagement with the human figures at all, and it appears they are facing away from the camera. The image is presented in low saturation, limited sharpness, and while lighting and contrast are prominent, these seem to hamper detail and obscure participants rather than be used in any aesthetic manner. The image can thus be said to be primarily naturalistic - an incidental view of the subject, taken from a largely unprivileged position of a distant, disengaged observer.

The second image shows a series of vehicles, with three human figures in the foreground - who seem to be wearing civilian clothing, as suggested by the man at bottom wearing a blue shirt, and men in the vehicles seemingly wearing grey chequered shirts - and another human figure on the right of the image, wearing what appears to be a military uniform, and facing away from the camera. The vehicles themselves are only seen partially, with the frame and other obstructions preventing a clear line of sight on their entire appearance, though sufficient attributes are seen to understand that these are military vehicles.

Although there appears to be a narrative built into this image, its nature is very confusing and difficult to interpret - with the text providing no real indications on its significance, aside from mentioning the Republican Guard's better situation in regards 
to equipment compared to the regular army, which is certainly evident in the picture. The workers in the foreground face toward the viewer, but the nature of their reaction or role is completely unclear. Perhaps they are involved in transporting the military vehicles. By being positioned on the opposite side and having opposite facing to what appears to be a soldier on the right of the frame, they seem to be distinguished from him - but the implications of this are not specified. Thus, if it is a narrative, it largely breaks down - which also suggests that this image is an incidental and naturalistic view of the subject, rather than a carefully-posed and arranged publicity photograph as those mostly predominant in portrayals of Coalition troops.

The low articulation of modalities such as contrast, saturation and sharpness certainly further indicates the naturalistic and unaltered, rather than carefullymanipulated sensory orientation of the image. The perspective is somewhat confusing, although given that the convergence points fall well outside the image, it can be said to be relatively oblique. With the vehicles largely obscured and the soldier facing away from the camera, there is both disengagement and distance suggested. The perspective, though relatively close, cannot be called privileged.

While the CNN's images of Iraqi troops take a particularly distanced approach, the image from BBC shown below assumes a different strategy.

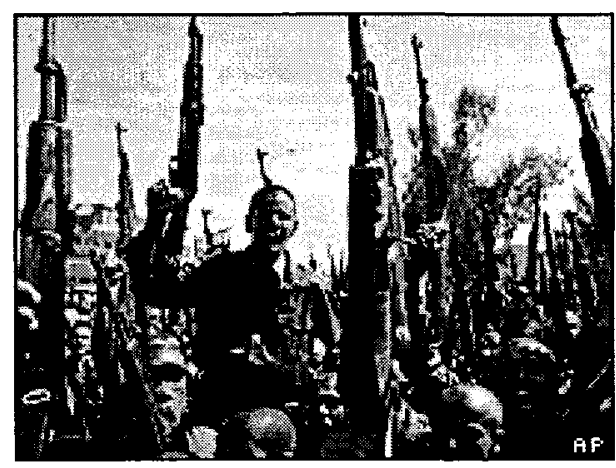

(2) BBC 
The soldiers represented in this illustration are shown in clear detail, with articulated facial expression and attributes such as guns distinctly visible. There is a narrative, reinforced by the subtitle which labels this as "Newly-conscripted Iraqi soldiers in Baghdad", suggesting perhaps a measure of readiness on the part of the Iraqi soldiers. This measure, of course, is highly qualified in the text - and evident even in the subtitle which seems to emphasize the conscripted and inexperienced nature of the troops.

What is most salient orientationally about this image is close distance, relatively low angle - especially relative to the guns, and aggressive expression of the troops. The latter is emphasized by the guns (which serve as circumstances of means or accompaniment) and significant repetition throughout the illustration. The image could easily be interpreted as directly threatening, placing the viewer in an insecure position. There is certainly engagement of the troops with the camera, but in the way it is construed - the engagement is perhaps an uncomfortable one. Otherwise, besides contrast, there is relatively low articulation of modalities and though the image has significant sensory effects due to prominent use of expression, it retains its primarilynaturalistic properties.

As the images above suggest, the portrayals of troops fall primarily under two patterns - extreme distancing and disengagement from the viewer, or conversely close engagement in a threatening manner. In both cases, the viewer is placed in relatively unprivileged perspective relative to the Iraqi troops. This pattern is generally retained throughout the data, wherever Iraqi soldiers are portrayed.

\subsubsection{Vehicles}

The depictions of vehicles in the BBC (2003) and CNN (1998) documents fall under mostly the same patterns described above, albeit there are more parallels to portrayals 
of Coalition vehicles as well. This is perhaps due to the inanimate nature of the subjects, which are of lesser importance in terms of alignment, since they are not as likely to elicit associations and reactions from the viewer as human subjects. Consider the images below:

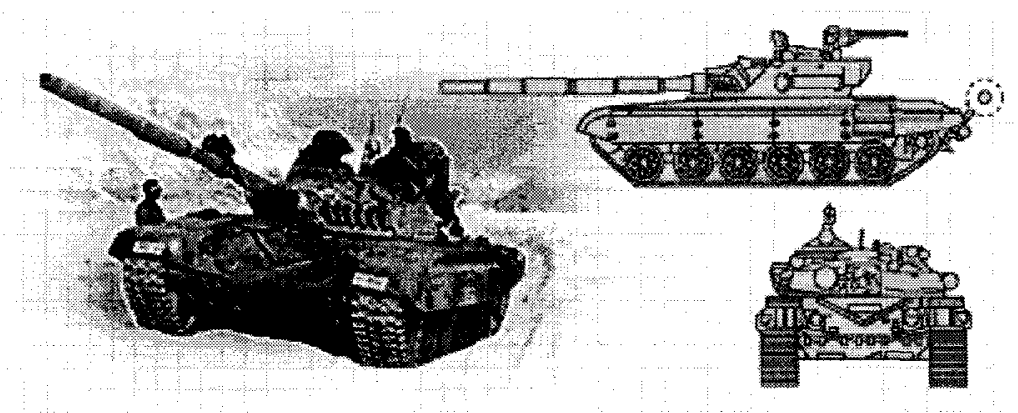

(6) $\mathrm{BBC}$

This primary illustration is largely parallel to the portrayals of Coalition tanks such as the Abrams seen previously. The differences include a more detailed, naturalistic background and a notably lower vertical angle than seen in similar illustrations for Coalition tanks, but the difference is not significant enough to be considered strategic. Certainly, the portrayal is still primarily sensory and suggests a privileged position though later in the same article, the text and secondary illustrations certainly seek to lessen any positive reactions to this tank.

However the patterns seen in the portrayal of troops are still very much at play - consider, for instance, the two images below.

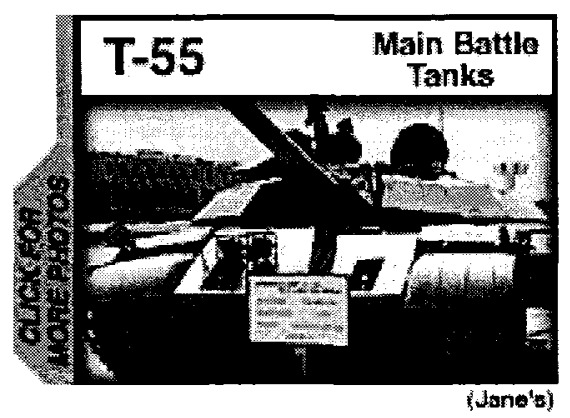

(4) $\mathrm{CNN}$

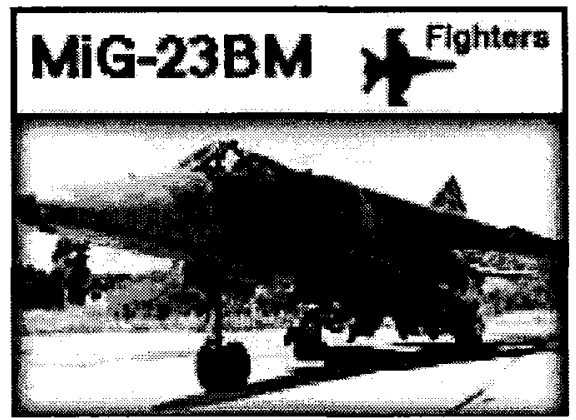

(7) $\mathrm{CNN}$ 
Both images show a single weapon system - a tank on the left, and a fighter aircraft on the right. Representationally, there is little of note in the image besides the participants themselves. Certainly, circumstances like the architectural style of the building in the first image (arches and columns at bottom, small windows at top) point to the Middle Eastern location, or simply indicate an airbase in the second image. One interesting item accompanies the tank on the left - a plate with information, which perhaps points to the fact that the tank is in a museum. This could conceivably be interpreted in a symbolic fashion, suggesting that the tank is fit to be a museum exhibit rather than a combat unit - which is certainly something that a reader may conclude from the text accompanying the image, which emphasizes that most Iraqi tanks are obsolescent, and "the Iraqi armed forces are in need of a complete range of modern equipment" (CNN, 1998). Whether it is of significance or not, it is worth noting that no Coalition units are shown in this fashion.

More crucially, no Coalition units are shown from the same perspective as the tank and aircraft in the above pictures. The units, instead of being shown from a medium distance, with their outlines approaching the edges of the frame but not exceeding it, are shown from a medium-close distance, and thus are shown larger than the frame. The angle for both units is frontal and relatively low, thus once again placing the viewer in an uncomfortably engaged, even threatened position. The images otherwise stay within relatively naturalistic limits and the perspective is a much less unusual one than those granted by airborne photography for the Coalition vehicles. Despite the closer distance, sharpness and level of detail are not very high. Thus while the images do have a primarily sensory effect on the viewer, they stay within limits of naturalistic orientation. 
The image below, meanwhile, parallels the distant and disengaged images of Iraqi troops which were discussed earlier.

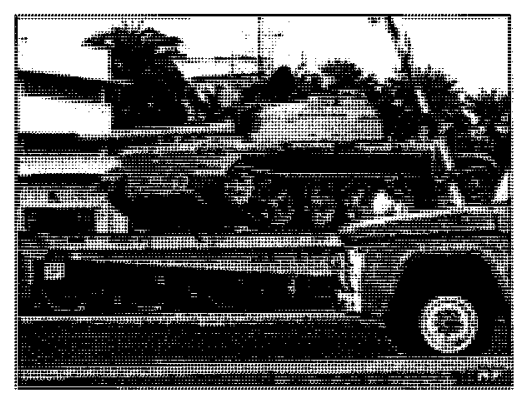

(5) BBC

This secondary illustration originates from the same article as image (6) discussed earlier. Here an Iraqi tank is shown from a medium distance, and would be central and seen in reasonably high detail were it not for the complex internal framing and unusually-detailed background which disperses the viewer's attention. The horizontals produced by the road and the railroad car, verticals produced by the building and the truck on the right, as well as the diagonal of the crossing marker complicate the framing and initially disorient the viewer. Further, the truck and crossing marker partially obscure the tank, placing a barrier between the viewer and the subject. The distancing is further enhanced by the rather narrow-angle view in this image.

The narrative structure is also somewhat confusing, although significantly aided by the subtitle, which states "Iraqi tank arriving in Baghdad by train on 17 March". The image is purely naturalistic in orientation, offering what appears to be an incidental view of a tank on the eve of the war, and using relatively low articulation of most modalities, which is particularly obvious from the low colour saturation. With the viewer placed in a distant, unprivileged position. This, contrasted with the privileged portrayals of Coalition units, creates an unfavourable portrayal of Iraqi capabilities. 


\subsubsection{Summary}

One can thus characterize two primary patterns predominant among the images of Iraqi troops and equipment: one is to place the viewer in a distant and highly disengaged position in relation to naturalistic portrayals of the subject with unclear narrative structure and relatively low articulation of modality; and the other is to present a frontal, low perspective of the subject at close range, placing the viewer in a disadvantaged and essentially threatened position. Although there are a number of deviations from these patterns, where Iraqi weapons and soldiers are shown in ways similar to those of the Coalition, they are certainly in the minority. With the use of these two strategies, meanwhile, both $\mathrm{BBC}$ and $\mathrm{CNN}$ help distance the viewer from the Iraqi forces and make him/her wary of their threatening disposition, thus implicitly aligning him/her with the Coalition. The negative positioning of the Iraqi forces also has a bearing on the interpretation of Section V, where biological and chemical weapons used by the Iraqis, as well as their effects, are presented.

\subsection{Section IV - Coalition Weapons}

The images analyzed in this section include those accompanying articles about various types of munitions used by Coalition forces and carried on many of the vehicles considered in Section I. It is in this section that a crucial strategic difference between $\mathrm{CNN}$ and $\mathrm{BBC}$ becomes most apparent. Consider the overall distribution described in the table below. 
Section IV - Coalition Weapons

\begin{tabular}{|c|c|c|c|c|}
\hline & Total & $\begin{array}{l}\text { Primary } \\
\text { Illustration }\end{array}$ & $\begin{array}{l}\text { Secondary } \\
\text { Illustration }\end{array}$ & $\begin{array}{l}\text { Exposition/ } \\
\text { Explanation }\end{array}$ \\
\hline CNN & 7 & 7 & & \\
\hline $\begin{array}{ll}- & \text { Ordinary } \\
& \text { Photograph }\end{array}$ & 2 & 2 & & \\
\hline $\begin{array}{l}\text { Publicity } \\
\text { Photograph }\end{array}$ & 5 & 5 & & \\
\hline BBC & 29 & 4 & 4 & 21 \\
\hline $\begin{array}{l}\text { Ordinary } \\
\text { Photograph }\end{array}$ & 2 & & 2 & \\
\hline $\begin{array}{l}\text { Publicity } \\
\text { Photograph }\end{array}$ & 2 & 1 & 1 & \\
\hline $\begin{array}{l}\text { - Scale Drawing / } \\
\text { Diagram }\end{array}$ & 6 & 1 & & 5 \\
\hline $\begin{array}{l}\text { - Function } \\
\text { Diagram }\end{array}$ & 13 & 1 & & 12 \\
\hline - Map & 1 & & 1 & \\
\hline $\begin{array}{l}\text { Optical Sensor } \\
\text { Data }\end{array}$ & 1 & 1 & & \\
\hline - Combination & 4 & & & 4 \\
\hline
\end{tabular}

Here one can see that $\mathrm{CNN}$ retains the same pattern of predominantly publicity photos used in the role of primary illustrations, thus presumably seeking to give the same type of sensory-oriented, distanced yet privileged portrayal seen in the images of Coalition vehicles in Section I. BBC, however, takes a drastically different approach that is uniquely dominant in this section: use of function diagrams and scale drawings, in other words technical imagery, in very predominant roles as expositions and illustrations. A few of these types of images do appear in other BBC sections as well, such as for example explanations accompanying certain vehicles, or a series of function diagrams describing the tactics of Special Forces troops, and certainly the observations made below are applicable to them as well. These abstract representations are a powerful way of controlling the imagery on several levels, and presenting what are essentially violent actions in a manner that is distanced, generalized, and makes no direct allusions to suffering - instead reducing the narratives to presentations of abstract processes acted out primarily by inanimate entities on inanimate entities. 


\subsubsection{CNN}

The patterns of imagery seen in CNN's (1998) articles on Coalition weapons are parallel to those used in the portrayal of Coalition vehicles. This includes the relatively close, privileged perspectives; high modality articulation; and use of simple, non-transactional narratives.

Consider the image below, demonstrating a primary air-to-air weapon used by the United States:

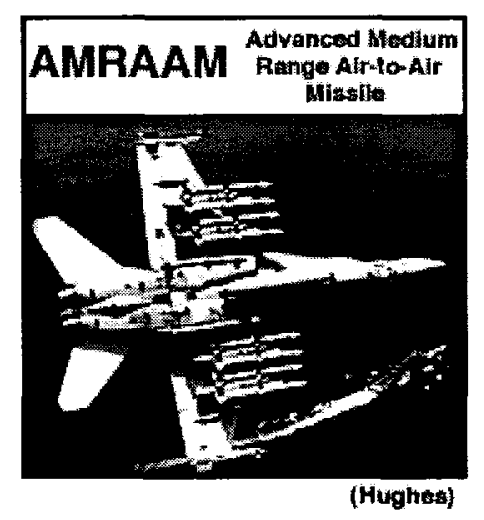

(5) CNN

As in many images previously discussed, it uses a two-frame structure, although the silhouettes used in the top frame for vehicles are absent. The photograph contains an aircraft, to which a number of missiles are attached, and a carrier turning in the sea below. The image is dominated by strong vectors, such as the orientation and shape of the aircraft and missiles which are geometrically symbolic of rapid movement, and the wake created by the ship in the ocean. The narrative in this image, however, emphasizes the relations between aircraft, missiles, and the ship - and the actions shown in the image are non-transactional. One could interpret the wake created by the ship and the unusual (partially inverted) orientation of the aircraft accompanying it to be circumstances of manner, and the relation between components in the image as circumstances of accompaniment. 
The perspective here is markedly unusual - with the camera obviously positioned high but the underside of the aircraft seen due to its dynamic position. The distance to the aircraft is medium, and the oblique horizontal image does disengage the viewer somewhat, also placing him in a safe and privileged position over the (inanimate) participants. The articulation of depth through positioning, sharpness, lighting and clear use of multiple levels is rather extreme, as are the general uses of lighting, contrast, and saturation. The image is meticulously posed and composed, with aesthetic elements such as colour, dynamic posing of participants, strong diagonals and refined composition maximally emphasized.

The two images below are likewise similar in a number of aspects:

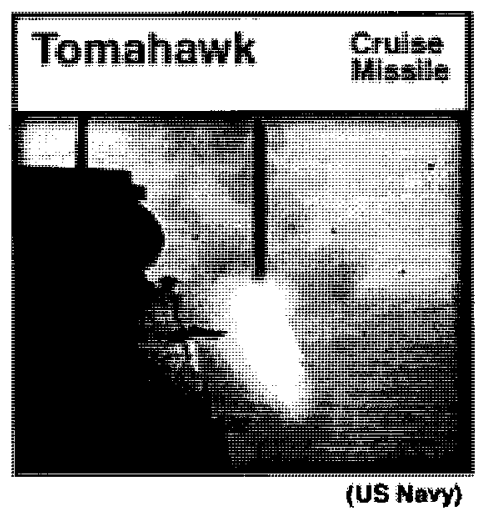

(1) $\mathrm{CNN}$

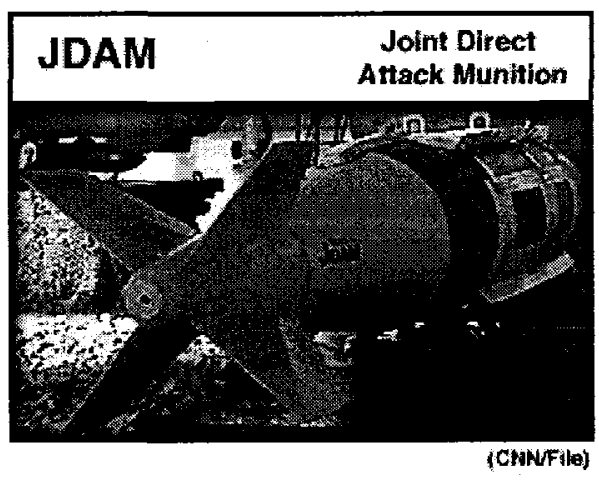

(3) $\mathrm{CNN}$

The first image shows a cruise missile being launched. The image is obviously taken from the ship which is launching the missile, and part of the ship's superstructure also creates internal framing within the image, helping to emphasize the missile. The perspective here is somewhat complex, and could be interpreted as partially frontal and medium relative to the ship, oblique and moderately low relative to the missile, and oblique and moderately high relative to the missile's trail of flame and smoke. Regardless, the position which the photographer occupies is a relatively privileged one: it is in close proximity to the weapons, which can only be attained 
with cooperation from military officials, allowing the viewer to see details of their operation and appearance in a way a distanced observer would not be able to.

The narrative produced by this image is a relatively abstract one and features a powerful material action (missile launching) - but it is not overtly transactional, with the object of the launch unclear. Meanwhile, the dramatic qualities of the photograph are emphasized through the complex perspective and sharp use of lighting and contrast, with extreme highlights and shadows, and a dramatic glint created on the side of the ship by the missile launching, against a relatively low-saturation, lowdetail background. Thus the image focuses the viewer on the attributes of the ship and the missile, and the aesthetic dimensions of the image such as sharp contrast, dramatic lighting, and unusual perspective.

The second image likewise offers a close and relatively privileged perspective of a Coalition weapon - a guided bomb, presumably shown in a storage facility. The center of the image is occupied by a label on the weapon itself clearly labelling it as a JDAM bomb, which helps clarify and organize the meaning of the image. With the background unclear, and no obvious processes or circumstances in place, the image simply emphasizes the appearance of the weapon. The bomb is shown at a close distance, and from a moderately high angle from the rear. With the bomb's fins at the front, suggesting that it is pointed away from the viewer, the image seems to follow the reverse of the frontal and moderately high angle used to show Iraqi weapons and vehicles.

As with images of Coalition vehicles, there is a notable absence of human agency or in fact any human participation in the CNN images of weapons. The patterns seen in the first section are thus largely continued in this case - with portrayals of weapons being largely focused on attributes of the weapons themselves, 
with only relational processes and non-transactional actions involved in the narrative structures of the images. The viewer is placed into privileged positions in relation to the subjects.

\subsubsection{BBC}

BBC's (2003) section dealing with the "Firepower" of the U.S. war on Iraq is substantially different from its equivalent on CNN (1998). Rather than continuing the patterns established in the portrayals of vehicles and troops, entirely new patterns are developed and new genres of imagery are engaged by the authors. Of particular importance are the technologically-oriented, non-photographic genres used here especially scale drawings and function diagrams. It is worth noting, of course, that a few of these types of images appear in other sections as well - although the numbers of such diagrams and drawings in sections on Iraqi weapons or Coalition vehicles are relatively few, and largely follow the patterns established within this section.

Statistics on their incidence can be found in the tables accompanying the introductions to each of the preceding sections.

Other notable features of this section are the only appearance of the optical sensor imagery outlined in the earlier discussion of generic types in section 4.1.1, as well as the only map in the data. There are also a number of photographs, used primarily as secondary illustrations, and combinations which combine features of photographs, diagrams and scale drawings. One of the articles, dealing with depleted uranium ammunition, includes a short animated presentation - which for the purposes of this study is simply treated as a combination of a series of scale drawings and function diagrams.

In explaining the entities most directly involved in the material processes of war, BBC's presentation is both detailed in its definition of attributes and processes, 
and at the same time highly abstracted and tightly controlled in the patterns of depiction. Dispensing with photography as the primary medium, the authors certainly use technical imagery to great effect.

\subsubsection{Topography}

Topographical structure is concerned primarily with accurate representations of spatial relations and dimensions - precisely the function of scale drawings as well as maps, examples of which are presented below.

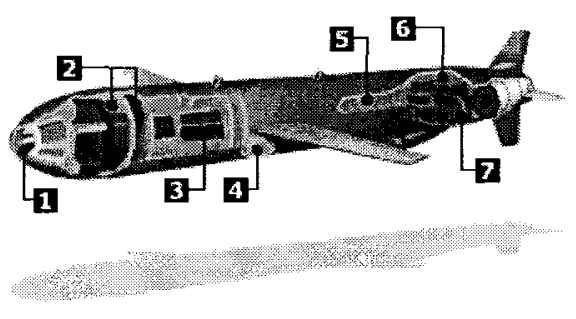

$(\mathrm{BBC})$

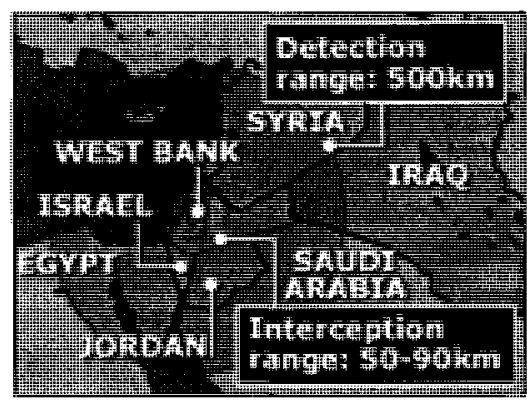

(BBC)

While only a single map is found in the entire data set, scale drawings are relatively widespread - and highly widespread if one includes the combinations seen in Section I (Coalition Vehicles) among their number.

In both images above, the function is essentially the same - to map various functions discussed in text onto physical reality. The difference is merely in scale and topic, as well as method of labelling - which is more heavily dependent on labels pointing to the text in the case of the first image.

Orientationally, there is little of note regarding the map shown in the second image - though certainly the use of color is of significance, that significance is still primarily functional - to indicate, for instance, involved countries with orange, uninvolved or neutral countries with grey, distances relevant to weapon systems with red, and water with blue. The choice of perspective and the choice of land area to 
frame corresponds purely to the representative demands of the subject, which is Israel's missile defense against Iraqi attack.

The scale drawing, however, certainly does feature some orientational elements motivated perhaps by factors outside representation - for example the use of isometric perspective and stylized lighting. This is still far from naturalism, but seems to mimic certain aspects of it. Likewise there is use of bright red and blue colours, to highlight explosive and navigational elements of the weapon respectively. The relatively close range and high, oblique angle, combined with the added access granted to the viewer afforded by the cut-away presentation, certainly suggest a privileged position - this is unlikely to be an arbitrary choice. Still, this is not to say that the image becomes sensory in orientation due to this aspect; certainly perspective and colour play a purely functional role foremost, and while the image could conceivably be without perspective, colour, and lighting - in the public media context, it would lose some of is functional clarity as a result.

What is crucial about the image, however, is that by drawing on inherently technological representation devices and producing topographical accuracy, it gains a certain claim to objectivity. Therein lies the strength of primarily technological orientations - they are structured in a way that focuses on factual information rather than sensory impressions or realistic appearance. In this way, despite being a stylized graphic, the image of the cruise missile in the first of the illustrations above provides more information and clearer presentation than the photograph of the same cruise missile being launched in CNN's (1998) article (see Image (1) in this section) - and, in effect, is a more objective depiction. 


\subsubsection{Topology}

Function diagrams are even more prominent in this section than scale drawings - and though the style of their presentation is very similar to that used in scale drawings, their structure differs fundamentally. Rather than being organized topographically representing accurately the spatial configuration of their subjects - these have a topological structure, which instead aims to accurately show logical relations between elements of the image. In the case of function diagrams, these depictions focus on representing stages and relations in material processes.

The image below shows the function of the British "Storm Shadow" cruise missile.

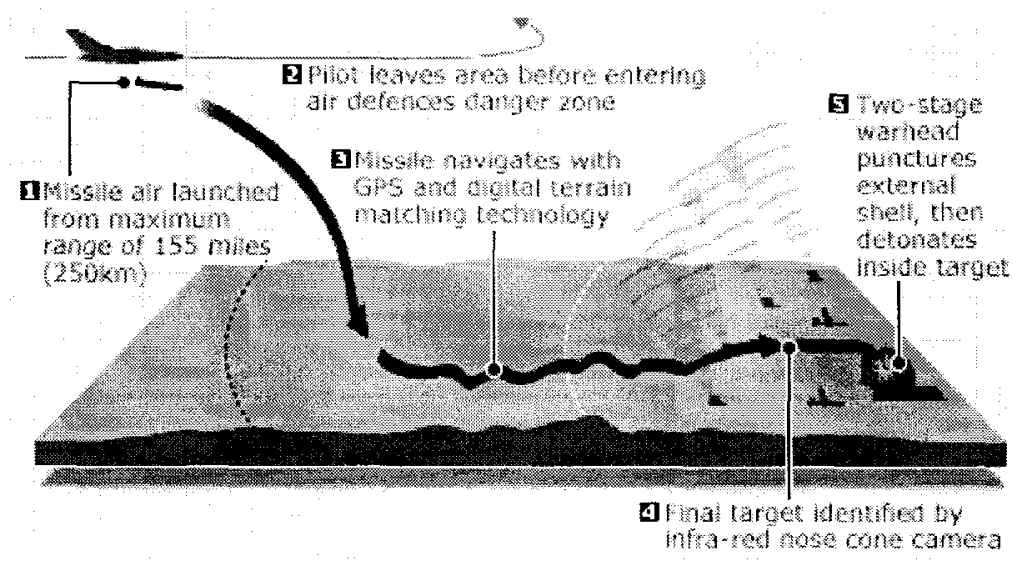

(2) $\mathrm{BBC}$

The first notable aspect of this image relates to the role in the article overall - which goes beyond simply that of primary illustration and its positioning as the generalized, idealized essence. It actually contains a substantial proportion of the information content on the page. In this case, one could say it is the text which is the illustration, fleshing out the details and helping clarify aspects of the narrative presented in the image. 
Organizationally, the primary frame of the image is formed by the light grey grid in the background. There is an internal frame formed by the terrain cutaway in the centre, which creates an abstract topography representing a desert landscape. The aircraft launching the missile is in the top left of the image, with a variety of objects representing Iraqi defenses and a dark grey box representing the target in the bottom right. One could infer a given-new relation from this, since the topic of the article is an air-launched missile, and thus it and the aircraft launching it are given while its functions like evading defenses and striking the target are the focus of the explanations - thus the new. This is likely also the motivation for predominance of left-right movement being indicated within function diagrams.

As already noted, the primary participants are the aircraft, the missile, a variety of Iraqi defenses, and the target represented by a dark grey box. The aircraft is a light grey outline, the missile is drawn in dark red, and defenses are represented by a variety of dark grey shapes. The most salient elements in the image however are the vectors - a series of dark red arrows indicating the missile's flight path, and a series of light grey cones with concentric curving lines indicating the radar coverage of Iraqi air defenses. While these latter are pointed up toward the aircraft's position, the vectors indicating the missile's path are split into three stages - a downward curve from the aircraft toward the ground showing the release stage, a series of uneven turns and curves showing the missile manoeuvring around terrain features, and finally a straight line curving sharply down at the end showing impact of the target, which is also highlighted by a stylized orange and red explosion. There is also a light grey arrow showing the aircraft's flight path as it flies forward then turns around. At every stage, these vectors are explicitly labelled and numbered to show the purpose and sequence of the various stages. Temporal structure is thus also important in the image. 
Unlike the photographic imagery discussed before, the narrative with its processes, participants and circumstances is very clearly specified and the material action is described in detail - though certainly selective and highly abstracted detail. Orientationally, the viewer is still placed in a privileged position - as suggested by the high vertical angle relative to the terrain cutaway, and also by the sheer specificity of the information presented to him/her via the vectors and labels. Beyond the landscape and the target, however, perspective is not employed and the representations of items like the aircraft, missile and defenses are two-dimensional. All representations are exceptionally small and lacking detail, although the sparse background and simple colour scheme allow them to remain clear and easily distinguishable.

Therein lies perhaps the key aspect of the image - while unlike photographic illustrations it shows a narrative of the entire operation cycle of a weapon, from launch to destruction of a target, it does so in an exceptionally distanced, abstract way. With the highly simplified representation, while the processes it may show are essentially violent, the viewer is given at best a vague reference to any form of suffering - as the final step of the process, the missile detonates inside the target, without mention of the consequences. All the entities acting and being acted upon in the image are inanimate, with the processes largely abstract and generalized in nature. It is difficult to sympathise with a 'target', or criticize a missile or even a pilot performing a mission. Thus, the viewer is guided towards an appreciation of the technical complexity and efficiency of the process rather than having to make any ethical judgement.

Another similar example of a function diagram, shown in a slightly different perspective, can be seen below. 


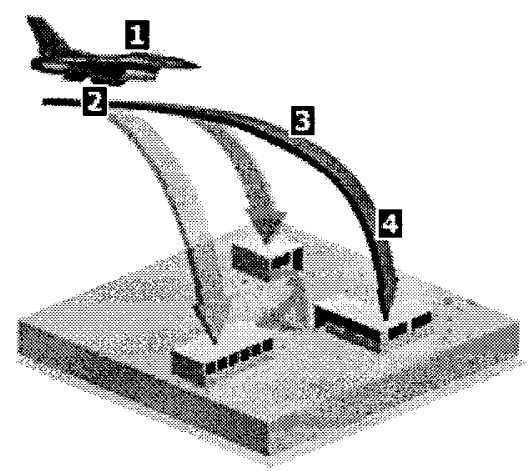

(4) BBC

The primary distinctions seen here from the above image are larger-scale representation of participants - including the aircraft and the buildings, which are slightly more detailed (although still retain very basic shape). At the same time, some of the detail seen in the previous image is also omitted, such as for example any terrain features beyond flat sandy ground, or much more importantly - the weapon itself or the explosion created as a result, which are not represented at all. The vectors are also much simpler and each of the possible weapon paths only includes one stage. The oblique horizontal angle here is emphasized, which further disengages the viewer. In this image the process is greatly simplified and turned into a completely abstract set of relations, representing what is in reality a highly complex and destructive set of material actions.

The ideological implication of this pattern of distancing, simplification and abstraction is perhaps even more obvious in the image below, which is the sole illustration in the BBC's (2003) Fact Files dealing with the issue of targeting errors by 'Smart Bombs'. 


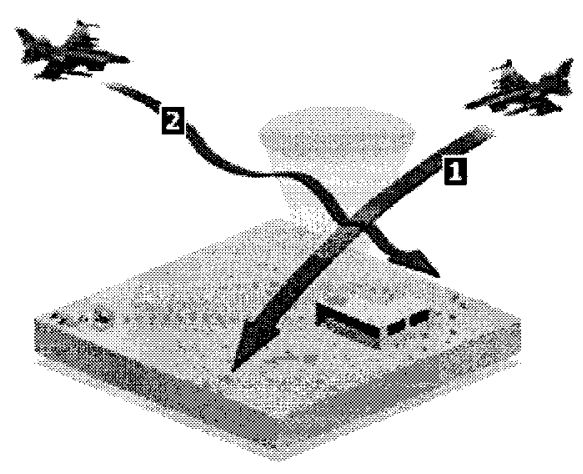

(8) BBC

The same style of presentation is used, with the stylized vectors showing the weapon paths, without either the weapon itself or its effects represented. Perhaps most curiously, the erroneous paths simply lead away from the stylized target and either into the open ground surrounding it or outside the frame. One of the arrows creating the path vectors is a relatively flat curve, while the other is irregularly curved. The error is thus marked clearly, but abstracted as far as possible and without any depiction or direct reference to consequences. Even in the text, consequences of errors are noted in very abstract terms - such as "The missiles cannot differentiate between a tank carrying soldiers and a tractor carrying civilians" (BBC, 2003). While the nature of the consequences can be inferred quite clearly, the avoidance of directly naming them is certainly not incidental.

The BBC's descriptions of weapons systems thus rely heavily on highly abstract, technical imagery that focuses on either topographic or topological structures. Due to the nature of the topic, these are highly controlled and qualified, allowing the authors to show complete representations, composition or function of highly destructive weapons while simultaneously drawing viewers away from ethical concerns with a high degree of abstraction. Despite the primarily technical orientation, certain elements of perspective are used - placing the viewer in privileged positions 
similar to those used in photographic imagery, and at the same time at a very long distance and high degree of disengagement from the material reality of the actions.

\subsection{Section V - Biological and Chemical Weapons}

The final section deals with the last set of subjects covered by CNN and $\mathrm{BBC}-$ the Iraqi weapons of mass destruction, biological and chemical weapons. In many ways this section serves as a counter-balance to the earlier ones, particularly on BBC. Having construed the Coalition role in the war as a set of largely abstract, distanced processes and at the same time having presented the Iraqi forces in an implicitly threatening fashion, as well as in a manner which kept the viewer particularly distanced from their position, the BBC (2003) presents a final section in its Fact Files which has a substantial pattern of images dealing with consequences of weapon uses suffering and victimization. Of the total of eight illustrations used, three of the images presented by $\mathrm{BBC}$ show victims, and four others show cleanup efforts and other associated imagery - for example symbols indicating fatal hazards. Although the agents of this victimization are not directly shown in the same images as victims, thus seeking to elicit pity from the viewer primarily, it is fairly clear who these agents are - Iraqi forces. Given that the primary cause for the outbreak of the 2003 U.S. war on Iraq had been the alleged possession of these weapons by Iraq, the inclusion of these weapons into the CNN (1998) and BBC (2003) documents is meant to encourage support for the Coalition's motives in conducting the war - so as to prevent suffering (potentially) caused by chemical and biological warfare.

The distribution of images in this section is presented below: 
Section V - Biological and Chemical Weapons

\begin{tabular}{|c|c|c|c|c|c|}
\hline & & Total & $\begin{array}{l}\text { Primary } \\
\text { Illustration }\end{array}$ & $\begin{array}{l}\text { Secondary } \\
\text { Illustration } \\
\end{array}$ & $\begin{array}{l}\text { Exposition / } \\
\text { Explanation }\end{array}$ \\
\hline CNN & & 1 & 1 & & \\
\hline - & Press Collage & 1 & 1 & & \\
\hline BBC & & 9 & 1 & 8 & \\
\hline & $\begin{array}{l}\text { Ordinary } \\
\text { Photograph }\end{array}$ & 5 & & 5 & \\
\hline & $\begin{array}{l}\text { Publicity } \\
\text { Photograph }\end{array}$ & 1 & & 1 & \\
\hline - & Press Collage & 3 & 1 & 2 & \\
\hline
\end{tabular}

Both BBC (2003) and CNN (1998) use press collages to introduce their sections on biological and chemical weapons. In the case of CNN, this is the only image in the section, with the rest of the section being simply a table with information on the weapons and their effects. $\mathrm{BBC}$, meanwhile, uses a series of articles, accompanied by illustration, to present information on the same weapons. The two introductory images are presented below.

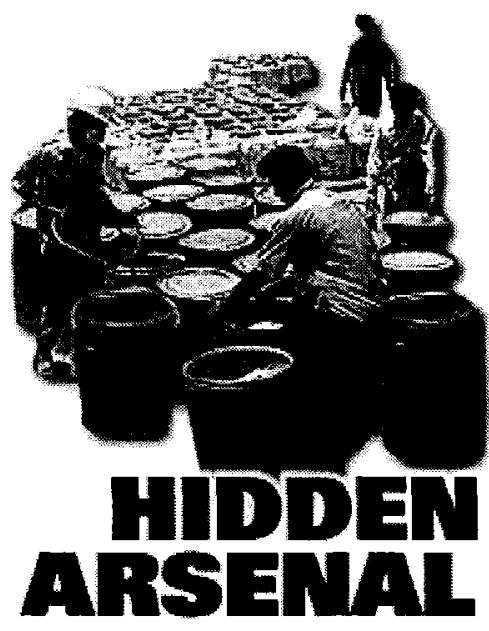

(1) $\mathrm{CNN}$

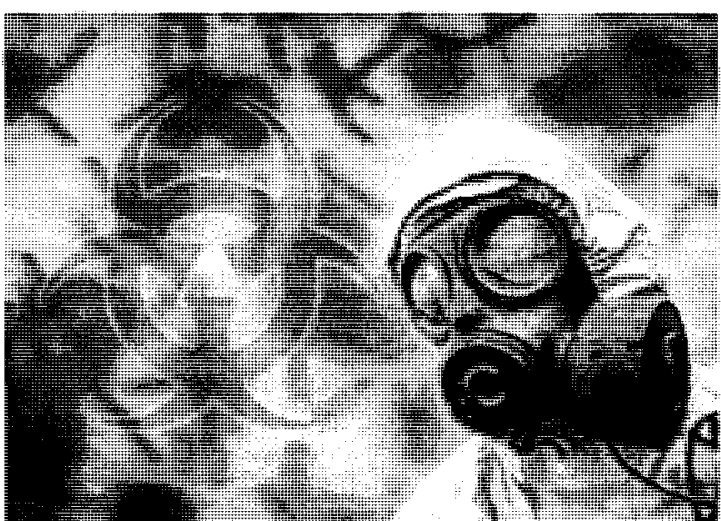

(2) $\mathrm{BBC}$

The CNN illustration seen in the first image includes a title and a photographic element with the background removed. The photographic element, showing people involved in some type of process with barrels of material, is above the title, thus serving as the ideal to the title's real - which is certainly a strategic choice, seeing that the image is certainly somewhat vague and could be open to other interpretation as to 
the actual nature of the narrative taking place in it. With the title, however, its interpretation and implication is significantly narrowed and made concrete.

The BBC's image does not include a title within it, however it is used in a very similar role in general. It is composed of three elements - a close-up photograph of a person in protective gear at the bottom right, a biological hazard symbol occupying the left side of the image, and a blurry red and yellow background which appears to be a microscope image of bacteria. The positioning of the elements in this case does not seem to have a particular information structure - rather, they are simply placed in the same frame to indicate their association with the same context and topic. Therefore the image is merely establishing an attributive symbolic relation between the element - and it is difficult to trace any particular narrative structure within it. Orientationally, the image takes a low and very close angle relative to the person in protective gear - a somewhat threatened position - which is perhaps to emphasize the dangerous nature of the elements in the entire image. With highly articulated colour saturation and a predominance of red - which can be considered symbolic of danger the purpose of the image is to reinforce the impression of extreme danger, which forms the essence of the subject.

CNN's image is quite different in regard to narrative and orientation. There are four men in the image, performing some type of operation involving barrels. A number of these barrels are seen in the foreground, with stacks of yellow crates or jars of some type stretching further back. The barrels positioned closest in the foreground are open, with yellow substance of some type visible in the leaning barrel furthest front. There is a man walking from the back of the image towards the foreground; the next furthest man seems to be moving one of the barrels; the man in blue shirt and hat in the foreground and left seems to be removing some type of seal from the barrel, 
and the man in the centre is performing some type of operation with an open barrel. In all of this there appears to be a back-to-front dynamic, reinforced by the use of perspective which sets the convergence point in approximately the middle of the image. With the title and the text alluding to Iraq's weapons programme, it is not difficult to read the image as a narrative of manufacturing, operating or perhaps even concealing weapons of mass destruction. The viewer is explicitly guided towards this reading, even though in the absence of the title and accompanying text, there could certainly be many other, less sinister interpretations of the narrative.

The photograph itself is taken from a moderately frontal and moderately high angle, from medium-long distance - although the depth of perspective is significantly hampered by the absence of background. The position could be judged as somewhat privileged, although the lack of engagement from participants indicates the observing position in this case to be relatively neutral. If the background were present, the image could be called a naturalistic, ordinary photograph, however it is removed in order to simplify and focus the image on the actions in the image which suggest some type of manufacturing process.

The following two images are used by $\mathrm{BBC}$ as secondary illustrations in articles on plague and nerve agents.

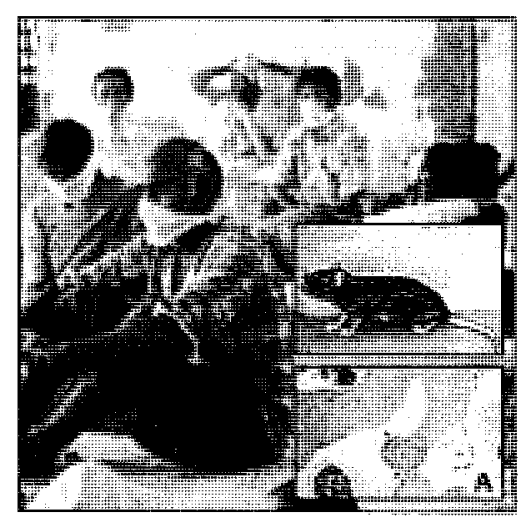

(3) $\mathrm{BBC}$

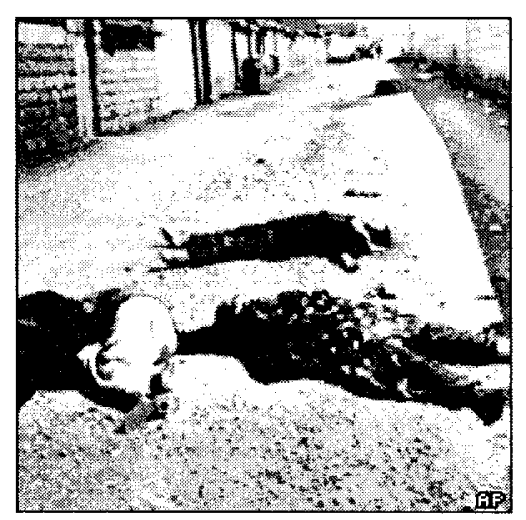

(4) $\mathrm{BBC}$ 
The first image uses three individual frames, with two smaller, higher-contrast and higher-saturation frames contained in a third, which extends to the entire image, but features a substantially lower modality. The smaller frames feature microscopic imagery of the bacteria which causes the disease, and a rat which can carry the disease; while the larger frame features people in some type of shelter, using improvised respiratory protection to guard themselves against the disease. The accompanying subtitle states that the photograph shows "Scenes during a plague scare in India" (BBC, 2003).

There are also some interesting orientational aspects in the main image frame. The perspective is from a relatively close distance, but uses an oblique angle, and the sharpness is substantially lower than the accompanying smaller frames, thus placing the viewer at relatively close social distance but also disengaging him/her to a large extent. Furthermore, the colour scheme appears to be shifted to yellow and brown tones in a way that is at least highly noticeable (and at most, intentionally altered), with the contrast relatively low. This could be described as a suggestive symbolic structure - creation of overall mood at the expense of detail. The bleak appearance and the concerned expressions of the people in the image certainly point to tension and a sense of danger that is intended to be associated with the subject.

The second image contains only a single frame, which shows a stark street scene with several dead bodies lying on the ground, indicated by the subtitle to be "Gas victims in the Iraqi town of Halabja" (BBC, 2003). The image has several highly striking orientational properties - including the high frontal angle and sharply articulated depth, which is enhanced by a very clear vanishing point within the image and strong diagonal lines leading toward it. The high-contrast, black and white depiction lessens the detail and sharpness to some extent, particularly for the dead 
bodies, but also creates a symbolically dark, striking atmosphere, underscoring the shocking nature of the image. Indeed the use of frontal, maximally engaging perspective is to draw the viewer in and elicit a reaction. At the same time, of course, the black and white colour scheme is also a barrier - helping to at least place the events in the image in a reality that is distant from the viewer's own realm of experience.

Another notable feature of the image is the absence of any overt agency - as noted earlier, while it may be obvious that Iraqi forces are behind the action, their role is not overtly presented - the victims are shown in isolation. This connection remains for the viewer to make. Like the 'regime of pity' described by Chouliaraki (2005a) in the portrayal of destruction caused by Coalition forces, "where suffering is construed as an object to watch and comment on" the goal in this case appears to elicit an affective reaction from the viewer, and encourage protest against biological and chemical weapons.

Chouliaraki goes on to ask in her (2006a) article, "Indeed, was it not in the name of pity, liberating the Iraqis from long-term suffering caused by his alleged Weapons of Mass Destruction, that the war was launched in the first place?" (p.264). Support for this action appears to be precisely the response sought in this case. Given that the primary justification for the 2003 U.S. war on Iraq was the alleged possession by Saddam Hussein's regime of these weapons, one can certainly see why the media would be seeking to show suffering or potential suffering inflicted through use of these weapons in a way quite unlike any other types of weapons discussed. 


\subsection{Summary}

In this chapter, I have outlined findings through data analysis, firstly noting broader patterns of distribution by generic types and functions of text, and secondly discussing the Representational, Orientational and Organizational features found in a narrower sample of selected images, broken down into five separate sections. A number of patterns, as well as notable exceptions, have emerged through these observations. The implications of these patterns, and overall conclusions from the above observations, will be discussed in the following chapter. 


\section{Discussion / Conclusion}

From analysis above, a number of important patterns emerge, many of them specific to individual sections and subjects to which the images relate. In Section I, Coalition vehicles are seen primarily in high-modality, single-participant publicity photographs that serve as primary illustration, offering the viewer privileged, aestheticallyappealing perspectives of the inanimate subjects at the centre of the discourse. The narrative structures in these images are generally de-emphasized in favour of composition and purely relational display of attributes and circumstances. There are a few exceptions in this section which do include human participants and simple narratives showing aspects of operations of these military vehicles, but the predominant mode of imagery in this section is sensory-oriented portrayals that focus on appearance and attributes, not processes.

In the Section II, Coalition troops are presented in a way that still employs a significant amount of high-modality imagery taken from privileged perspectives, but the narrative structures and relations built between the subjects and the viewer are much more complicated, to suit the overarching purpose of aligning the viewer with the troops but distancing him/her from the violent nature of the functions they perform. Thus most narratives remain focused on non-transactional processes - with soldiers shown marching, aiming or training but not directly engaging in combat or acting directly on other participants (with a single, highly marked exception to the pattern). Where it is advantageous to place the viewer at a closer social distance from the participants in the image, closer and more frontal perspectives and articulated facial expressions are provided. Where the viewer needs to be distanced, a variety of devices such as oblique perspective, obstructions and longer distances are employed. Part of the variety seen in the imagery is also motivated by the fact that unlike Section 
$\mathrm{I}$, most of the images used in this section by BBC are secondary illustrations, serving in a more supplemental role relative to the text and illustrating individual features of their subjects rather than a generalized essence. Within this section, the primary aim appears to be building favourable - if distant - relationships between the viewer and the subjects of the discourse (soldiers).

In Section III, Iraqi forces and vehicles are shown in a substantially different way than their coalition equivalents. Using lower-modality, more naturalisticallyoriented imagery, the $\mathrm{BBC}$ and $\mathrm{CNN}$ also place viewers in less privileged positions relative to the troops - and occasionally in threatened positions, as indicated by the low frontal angles at close distances, which are not seen anywhere else in the discourse. The viewer is maximally distanced and aligned away from the Iraqi forces in most of the images, which naturally draws him/her towards the Coalition's perspective.

In Section IV, a significant divergence is seen between $\mathrm{BBC}$ and $\mathrm{CNN}$ in the ways they present Coalition weapons. $\mathrm{CNN}$ follows largely the same pattern as established in Section I, using publicity photographs as primary illustrations to place viewers in relatively privileged positions, and focus on appearance and attributes of the weapons. BBC, however, employs technical drawings and diagrams in a substantial majority of weapon portrayals. Function diagrams in particular are used to produce narratives of the weapons' operations - albeit in a highly controlled and abstract fashion, allowing the authors to be highly selective in terms of criterial aspects of the discourse. In this manner, the diagrams avoid any considerations of ethics, of destruction or suffering inflicted by the weapons - since almost universally, the weapons act upon highly stylized 'targets', simple geometrical shapes representing buildings. All actions and interactions in the discourse are framed as 
stemming from inanimate subjects acting upon other inanimate subjects. Thus, within the discourse, the viewer is not given an opportunity to criticize what is taking place from an ethical standpoint.

In Section V, which deals with portrayals of chemical and biological weapons, there is a curious reversal - with the images overtly showing consequences of weapons use, human suffering and death. Though the narratives are articulated to a minimum extent and overt portrayal of causality is at best blurry, most viewers will know from the context that these weapons are something which Iraq is accused of possessing, and thus agency or potential agency is implied very strongly.

Thus, both $\mathrm{BBC}$ and $\mathrm{CNN}$ guide the viewer towards a perception of the war as a whole through a series of stages. The viewer is aligned with Coalition forces largely by being placed in privileged positions relative to them, and the highly detailed, carefully composed nature of the imagery associated with the Coalition suggests a sense of order, a sense that the military is capable of handling the situation. Their methods are largely precise and the weapons are suggested to be highly capable. By contrast, images of Iraqi forces place the viewer in a doubtful, unprivileged and threatened position. With weapons of mass destruction implying the consequences of inaction, one could certainly interpret the entire document sets on weapons and forces by BBC (2003) and CNN (1998) as not simply favouring presentations of Coalition forces and weapons, but in fact as calls to action, actively building support for military solutions to tensions with Iraq.

\subsection{Privileged Positions, Generic Types}

One theme that has emerged in the analysis is the divergent portrayals of Coalition troops, vehicles and weapons and their lraqi equivalents in the CNN (1998) and BBC 
(2003) documents. Even on the level of generic types, this divergence is very apparent. Consider the distributions shown below:

\section{Coalition Forces and Weapons, by Generic Type}
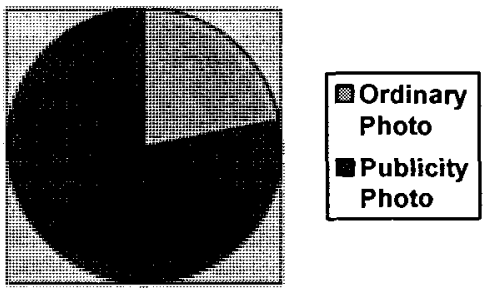

CNN
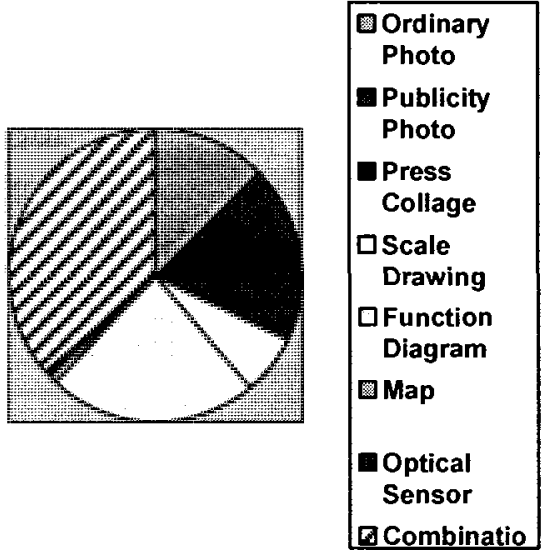

BBC

In the portrayal of Coalition forces, one can see that the $\mathrm{CNN}$ shows an overwhelming predominance of publicity photos. Most of these, as has already been noted, are images taken from privileged perspectives, and fall under primarily sensory coding orientation - high modality imagery with an aesthetic appearance and largely promotional purpose. The $\mathrm{BBC}$ employs a much greater range, with the three most common types of images of Coalition troops being publicity photographs, function diagrams, and combination images - which generally combine publicity photographic elements and scale drawing elements. Thus, sensory coding orientations still play a significant role - although there is greater balance and variety in BBC's overall visual presentation. This does not, however, translate into a more ideologically-balanced perspective, since the technical elements actually permit a greater degree of abstraction and control over modality than publicity photographs. This lack of balance certainly becomes apparent when one considers the distribution of the same generic types for Iraqi forces: 
Iraqi Forces and Weapons, by Generic Type

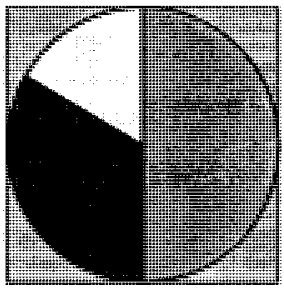

\begin{tabular}{|c|}
\hline Ordinary \\
Photo \\
Publicity \\
Photo \\
Q Function \\
Diagram \\
\hline
\end{tabular}
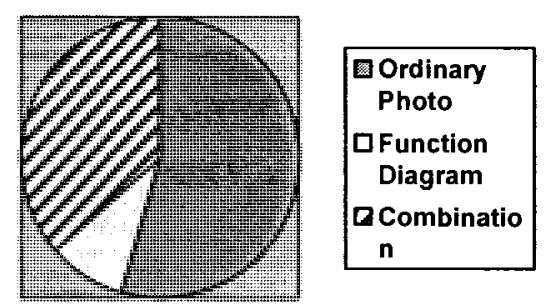

CNN

BBC

Here the ordinary photographs are predominant in both $\mathrm{CNN}$ and $\mathrm{BBC}$ articles. This echoes the patterns of lower modality and less privileged perspectives observed in detailed analysis of individual pictures. Whereas the Coalition forces and weapons are portrayed in a predominantly sensational fashion, Iraqi forces are presented in much more ordinary ways. And certainly, if the numbers of photos are any indication, the Iraqi forces are treated as a far less interesting subject by $\mathrm{BBC}$ and $\mathrm{CNN}$ overall, and thus only a small portion of the total number of articles and photos are dedicated to them - another expression of the implicit alignment.

\subsection{Coding Orientations, BBC vs. CNN}

As already noted above, modality plays a significant role in shaping the orientation of the viewer in the discourse. In this context, the use of high-modality and technological configurations has been already shown to correlate with the efforts to align the viewer, especially in the articulation of perspective. However the juxtaposition of different coding orientations has also proven to be very productive in aligning the viewer through contrasting use of modality. 
Coding orientations, as outlined earlier, fall under three relevant categories in this context - Naturalistic, Sensory and Technological. While there is a variety of ways in which they can be mixed in individual images, one can certainly draw conclusions regarding predominant coding orientations in each of the generic types discussed. Ordinary photographs can be said to fall under primarily Naturalistic coding; publicity photographs, press collages and sensor imagery (as used in the context) fall under primarily Sensory coding; while scale drawings, function diagrams and maps use Technological coding orientations. Combination imagery, as used in this context, uses both sensory and technological orientations in a complimentary fashion, without either predominating. Recalling the earlier summary of the distribution of genres, consider the summary below:

\section{Primary Coding Orientation}

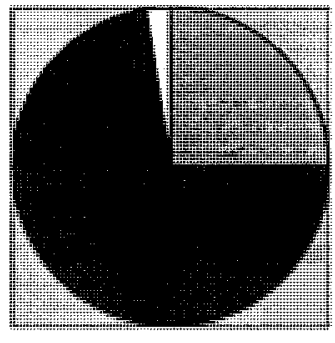

CNN

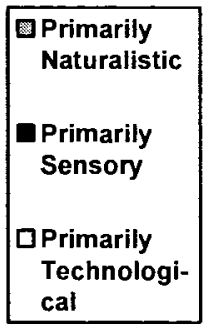

cal
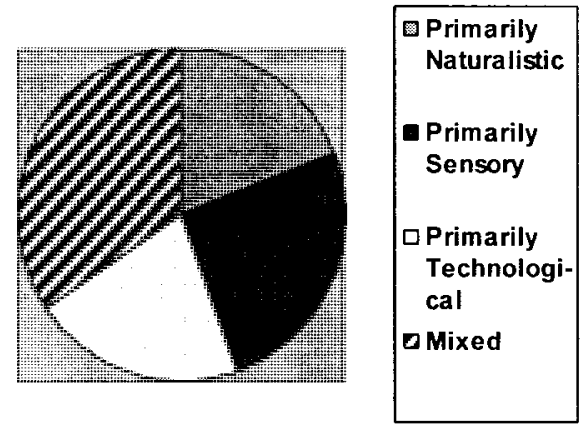

BBC

From this analysis, the difference between $\mathrm{CNN}$ and $\mathrm{BBC}$ is highly evident. CNN's (1998) presentation relies overwhelmingly on sensory orientations, while BBC uses a much more balanced set of orientations. The substantial part of this difference lies in the use of technological orientations by $\mathrm{BBC}$, something that is nearly absent on $\mathrm{CNN}$.

One way of accounting for this difference is to consider the rhetorical demands and goals of the two outlets. CNN, being an American media company, had probably faced a more favourable audience overall - and certainly in 1998 there had not yet been any significant criticism of continuing military pressure on Iraq. One could 
assume that the $\mathrm{CNN}$ sought less to realign its viewers on the topics discussed, and more to support the consensus. Thus the simpler, more sensory-oriented strategy suited their demands - providing a largely sensational portrayal of the forces involved, and giving the viewers an impressive portrayal of Coalition machinery and its attributes. BBC's (2003) Fact Files had been published at a time when the company's primary audience, the British public, had been relatively sceptical of large-scale military action against Iraq. Thus the BBC deployed a more comprehensive strategy that balanced sensory portrayals with technological ones - in effect assuring the public that the weapons and forces of the Coalition not only looked impressive (especially compared to those of the Iraqi side), but also functioned in ways which were technically sound and well-understood.

As a result, BBC's (2003) presentation is both more comprehensive and more persuasive. By employing a wider and more balanced range of coding orientations, the Fact Files gain a greater impression of thoroughness, objectivity and impartiality - something that the more sensational portrayal used by CNN lacks. Of course, the impression itself is largely misleading - which certainly providing more technical detail, the $\mathrm{BBC}$ remains faithful to the same ideological goals of aligning the viewer with the Coalition, and distancing him/her from ethical concerns over the conduct of the war.

\subsection{Strategic Patterns}

From the results of the analysis of illustrations included in the documents describing weapons and forces of the Iraq conflict on CNN's (1998) and BBC's (2003) websites, I would suggest that there are four major, inter-related patterns that guide the specific depiction strategies in groups of individual images. 
1) alignment - the positioning of the viewer in support of the Coalition in the Iraq conflict, and against the Iraqi forces in the conflict

2) distancing - the positioning of the viewer at a discursive distance from the events portrayed by the images, particularly those which involve violence

3) abstraction - the reframing of material actions which constitute the war as abstract, timeless processes carried out primarily by inanimate or diffused entities

4) aesthetization - the focus of the presentation on the aesthetic features and attributes of its subject, rather than its functions

I would suggest that these patterns are a way of positioning the viewer in a manner that maximally diverts his/her attention from violence and suffering. This in itself is no doubt a strategic, ideologically motivated choice - the motivation for which will be discussed in Section 5.1.4, further below. Before proceeding to the ideological considerations behind these patterns, I will specify in greater detail what these patterns imply, how they are manifested throughout the data, and how they interact with each other.

\subsubsection{Alignment}

As already discussed earlier, images of Coalition forces and weapons use several techniques to align the viewer with their subjects - which include munitions, vehicles, soldiers, and friendly militias. This is accomplished primarily through the system of perspective, by placing the viewer in privileged positions relative participants in the image - and to a lesser extent, through high articulation of modalities such as detail which symbolically enhances the sense of proximity to the subjects. Patterns of privileged perspective and high modality (which is inherent to publicity photographs) are predominant in Sections I, II and IV of the Analysis, both on BBC and CNN, although in slightly different ways - for instance, as noted above, BBC's reliance on technological in depictions of Coalition weapons (Section IV) and a widespread use 
of combination imagery which includes elements of technical drawings, produces a different type of privileged position from those seen in publicity photos - that of an observer provided with access to technical information.

There is, of course, an inverse to this - while the viewer is aligned with the Coalition position, he/she is simultaneously aligned away from (or in opposition to) Iraqi forces. This is expressed through either distant, disengaged, thoroughly unprivileged perspective - or high frontal perspective at close distance, which as earlier discussed, suggests threat. Given the high consistency of patterns of unprivileged perspective, this complimentary pattern emerges very clearly.

Alignment is used as a strategy for legitimating the conflict, with Coalition troops and weapons suggested to be favourable to the viewer's safety and close to his/her interest.

\subsubsection{Distancing}

The pattern of distancing is also accomplished largely through perspective; it is used to distance the viewer from depictions of processes involving violence, and from the recipients and agents of this violence. This is most evident in the function diagrams on $\mathrm{BBC}$ which are drawn with participants taking up only a small portion of the size of frame. This is likewise evident in some of the depictions of troops. Distancing seems to go hand in hand with disengagement - and certainly, a large portion of the images of both Coalition and Iraqi units use oblique perspective. Again, this is most obvious in function diagrams, which are almost universally presented from oblique angles.

The effect is to position the viewer outside of the immediate context of war, and unless he/she is encouraged to react positively or negatively for the purpose of 
alignment, keep him/her disengaged from the actions, maintaining the role of an observer - perhaps an aligned one, but not a directly involved one. This is a strategy to give the viewer a sense of security - suggesting that this security is best protected by those weapons and forces that he/she is aligned with. It also removes him/her from the context of violence and suffering, thus precluding sympathy for victims of the weapons, forces and processes taking place. Both BBC (1998) and CNN (2003) employ this pattern.

A notable exception to that, of course, is seen in Section V, in regard to Chemical and Biological weapons. Here, the viewer is intentionally brought closer to the site of suffering in order to encourage condemnation of the perpetrator - which happens to be the Iraqi regime, and thus the exception to the pattern of distancing from suffering serves to reinforce the pattern of alignment.

\subsubsection{Abstraction}

The pattern of distancing generally coincides with patterns of abstraction and representation of violence in ways that are implied, highly stylized (such as diagrams) virtual absence of transactional material actions performed by animate actors. Lack of animate agency is a theme that is retained through virtually all sections except those dealing directly with troops, where it is unavoidable. Photographs of ships, aircraft, weapons and tanks rarely feature any human participants, both on $\mathrm{BBC}$ and $\mathrm{CNN}$.

This also applies to the depiction of processes, which are very rarely transactional in nature, showing mostly non-transactional actions such as movement and walking, or no material actions at all; only one image of those examined shows a clearly distinguishable act of violence (recall Image (7) in Section II, where an 
Australian Special Forces soldier subdues a terror suspect) shown in naturalistic, concrete terms.

Nowhere is abstraction more evident than in the use of function diagrams by BBC (2003) to illustrate the operation of weapons. In that particular case, actions which in reality are extremely violent and destructive are represented as concepts through topological structure, using stylized vectors and construing the event taking place as a relation between an aircraft, a weapon, and a 'target'. Naturally, the target is itself an abstract representation of an inanimate object (a building), and thus cannot be subject to suffering.

With the exception of the above-mentioned image of the Australian Special Forces soldier, the actions of Coalition troops and weapons preclude concrete, animate goals - only 'targets'. Recalling the pattern observed by Chouliaraki (2005), it is certainly true in this discourse that the sufferer only enters the discourse "on the condition that her very humanity is cancelled" (p.155) - to be replaced by an abstract representation. This applies particularly to the BBC's (2003) depictions, if only because in CNN (1998) documents, the sufferer does not enter the scene at all.

The human agents, however, are only marginally more specific. Most of the time, human participants enter this discourse not as individuals, but as representative of types - 'generic people' as Machin (2004) refers to them.

\subsubsection{Aesthetic Appeal}

The use of sensory coding orientations to portray Coalition troops and weapons is particularly predominant on $\mathrm{CNN}$, which favours the use of publicity photographs in primary illustration roles in all instances where Coalition forces are presented. This focuses the viewer's attention on the external attributes of weapons, enhanced by the 
use of high modality, which articulates aspects of colour, depth, sharpness and detail. The use of privileged perspectives in both CNN (1998) and BBC (2003) images provides markedly unusual, detailed views of their subjects (for instance, through use of aerial photography). These images are carefully composed and framed, with subjects (such as soldiers, tanks, aircraft and ships) shown in dramatic poses/positions. The use of sensory orientations also rarely coincides with transactional material actions, meaning that narrative aspects of the image are de-emphasized in favour of attributes and orientational properties. In other words, by focusing on the appearance and attributes of a subject, the image strategically deemphasizes its material role. Given that the material role of Coalition weapons is an essentially violent one, this pattern is certainly strategic and has ideological implications. As Chouliaraki (2005, 2006a) suggests, by framing events and subjects as aesthetic, authors of the discourse invite the viewer to observe them as participants in an impressive performance rather than in acts which constitute suffering.

\subsection{Culture of War and Technology}

Given the observations regarding the patterns noted above, it is certainly possible to draw some general conclusions regarding the underlying ideologies responsible for these depictions, what Chouliaraki (2005) refers to as "the assumptions already implicit in the routine professional choices that stage and narrate the war" (144).

The antecedents to the pattern of alignment and aesthetization in regards to Coalition troops and weapons are not difficult to surmise: it is apparent throughout the visual discourse in the CNN (1998) and BBC (2003) documents that they seek to portray the Coalition in a more positive, legitimate and appealing light. This forms one half of an 'us and them' situation - and the use of unprivileged and/or threatened 
positions relative to the Iraqi forces, as well as their implied involvement in the use of Chemical/Biological weapons discussed in Section V of the Analysis, makes it very clear that they represent the other side. The aesthetically appealing portrayal which emphasizes the impressive appearance of Coalition weapons and soldiers, versus the unprivileged portrayal of the Iraqi forces also suggests which side should be considered the more capable one.

Distancing and abstraction are used to draw the viewer away from the actual nature of the events and actions taking place in war - violence and suffering. The images used by CNN (1998) and BBC (2003) contain no allusion to any suffering caused by Coalition forces. As discussed at the end of Section IV of the analysis, even targeting errors are framed in terms of misses, in visual terms. There is no grounds provided visually in the discourse to make ethical judgments about the role of weapons and forces in the war, which shifts the viewer's attention to technical and aesthetic dimensions of the depiction. The result, as Chouliaraki (2005) suggests in her study, is that "suffering is construed as an object to watch and comment on, rather than to arouse emotion or a demand for action on the part of the spectator" (p.151). In other words, the ideological goal of these patterns of depiction is to deflect criticism of war by omitting depictions of suffering caused by Coalition forces.

Recalling the brief discussion of the history of media portrayals of modern war provided in Section 3.6.I, it is easy to parallel these goals with observations made by Andersen (2006) and Lewis (2007) regarding the efforts of the media to emphasize the role of technology in war, which has been a recurring theme in Western, particularly American approaches to war in recent times. As Andersen (2006) noted about the portrayal of the 1991 Gulf War, "High-technology weaponry became the answer to issues once raised about the moral and ethical - or even economical - 
problems of war" (p.164). Certainly, both BBC (2003) and CNN (1998) prominently emphasize the role of technology in their representations. This coincides with what Lewis (2007) suggests is not only part of media depictions, but a much broader cultural ideology pioneered by the U.S., which for decades has been investing into military technology to perfect a 'cleaner', more distant and 'machine-driven' concept of war: "The United States is working tenaciously to eliminate man from war, to kill with technology from a safe distance, to fight war without casualties" (p.399). There is tremendous investment and political will behind this concept of war, and thus it is the powerful ideology of technological solutions to ethical problems of war - however unrealistic it is - which likely serves as the primary antecedent to the way in which powerful, mainstream Western media such as $\mathrm{CNN}$ and $\mathrm{BBC}$ present war and its weapons.

\subsection{Limitations and Directions for Further Study}

In this study, I have outlined a number of patterns from an extensive set of visual data, and showed how these connect to ideological antecedents. The methodological framework adapted for the study proved productive, and produced a qualitatively rich description of the discourse.

Some of the limits of the present study have already been noted earlier - for instance, the full range of data found in the CNN (1998) and BBC (2003) documents simply could not be presented due to space restrictions. While I have made an effort to outline salient exceptions and variations to overall patterns, there is certainly a risk of overstating some of these.

The exclusive focus on static visual illustrations certainly does not capture all of the meanings made in the CNN (1998) and BBC (2003) documents, and I certainly 
make no claims regarding the discourse on weapons and forces of the U.S. war on Iraq presented on $\mathrm{BBC}$ and $\mathrm{CNN}$ as a whole - only the aspects of it found in the visuals accompanying this specific set of documents. To give an account that would approach a fuller description of a wider discourse of war in these media outlets, a more thoroughly multimodal approach is needed. The analysis presented here includes some multimodal considerations, mostly through describing the immediate relations between image and text, with images being used in a variety of illustrative roles. Much of the literature examined for this study, including Lemke (1998, 2002, 2004, 2005), Iedema (2003), Kress and van Leeuwen (2006), Royce and Bowcher (2007) and Knox (2007) calls for more thoroughly multimodal accounts which examine all types of semiotic modalities found in discourse. Certainly it would be interesting to extend this study to consider the articles which these images accompany, using SFL methodologies referred to in this study (Halliday and Mattheissen, 2004; Eggins, 2004), and examine more specifically the ways in which 'intersemiotic complimentarity' (Royce, 2007) is realized. An initial look suggests that just as the depiction patterns between different sections of the data vary, so do the patterns found ideationally, interpersonally and textually in the accompanying articles. It would be interesting to pursue a more complete multimodal analysis on this topic.

Lemke's (2002) work also offers possibilities of extending this work into a Hypermodal direction, examining ways in which non-linear structure and traversals influence interpretations of extended media documents such as this. Perhaps even more interesting is Lemke's $(2004,2005)$ work on the concept of traversals and 'transmedia'. For instance, it would be possible to compare the depiction patterns found in this text to those of the same weapons and forces found in other types of media, including films and interactive video games - and consider to what extent 
similar patterns emerge, and to what extent resemiotization (ledema, 2003) would play a role in the type of depictions across media.

Lemke (1998) also makes reference to a system of evaluative meanings which could significantly augment orientational patterns discovered in the data by suggesting not only how the viewer is oriented, but also what positive or negative evaluations he/she can draw from the positioning of the subject. This would be particularly helpful in further extending the discussion of patterns of alignment, distancing, abstraction and aesthetization outlined above.

Finally, with the 'new media' continually developing, visual and multimodal features that are yet to be fully explored are emerging on news websites. $\mathrm{BBC}$, as noted before, has recently introduced embedded video, which is now used to illustrate current news articles in much the same way as static images are used in the (2003) Fact Files examined in this study. As Mautner (2005) suggests, internet media features an increasing amount of interactive and user-created content, and news websites are increasingly adapting elements such as user feedback forums and interactive games which relate to the news stories being presented. Certainly, the present study only begins to deconstruct the full semiotic potential of extended online media features.

\subsection{Summary}

This study has thus considered the ways in which the visuals are used by the CNN (1998) and BBC (2003) documents on weapons and forces of the U.S. war on Iraq. Using a functional framework of visual analysis based on the work of O'Toole (1994), Lemke (1998, 1999, 2002, 2004, 2005), Oyama and Jewitt (2001), Iedema (2003), Machin (2004), Teo (2004), van Leeuwen (2005), Kress and van Leeuwen (2006), Royce and Bowcher (2007) and Knox (2007) and others, I have described a set of 
representational, orientational, and organizational patterns in depictions, discussing general tendencies and drawing ideological implications. The results of the study suggest that predominant patterns found throughout the text are used to produce favourable portrayals of Coalition weapons, unfavourable portrayals of Iraqi weapons, and to position viewers in a way which distances them from material suffering. 


\section{References}

Allan, S. \& Zelizer, B. (2004). Reporting war. London: Routledge.

Andersen, R. (2006). A century of media, a century of war. New York: Peter Lang Publishing.

Bakhtin, M. (1981). The dialogic imagination. Austin: University of Texas Press.

BBC (2008). BBC heritage. Retrieved July 29, 2008, from:

http://www.bbc.co.uk/heritage/story/index.shtml

Boczkowski, P.J. (2004). Digitizing the news: Innovation in online newspapers. Cambridge, MA: MIT Press.

Boltanski, L. (1999). Distant suffering: Politics, morality and the media. Cambridge: Cambridge University Press.

Bowcher, W. (2007). A multimodal analysis of good guys and bad guys in Rugby League Week. In T. Royce and W. Bowcher (Eds.), New directions in the analysis of multimodal discourse (pp.239-274). London: Routledge.

Chilton, P. (2004) Analyzing political discourse. London: Routledge.

Chouliaraki, L. (2004) Watching September 11: The politics of pity. Discourse and Society, 15 (2-3), pp.185-198.

---- (2005). Spectacular ethics: On television footage in the U.S. war on Iraq. Journal of Language and Politics, 4(I), pp.143-159.

---- (2006a). The aestheticization of suffering on television. Visual Communication, $10(5)$, pp.261-285.

---- (2006b). The spectatorship of suffering. London: Sage.

CNN (2007). About CNN. Retrieved July 29, 2008, from: http://www.cnn.com/about/

Cooke, L. (2003). Information acceleration and visual trends in print, television, and web news sources. Technical Communication Quarterly, 12(2), pp.155-81.

Eggins, S. (2004). An introduction to systemic-functional linguistics. $2^{\text {nd }}$ Edition. London: Continuum.

Fairclough, N. (1992). Discourse and social change. Cambridge: Polity Press.

-..- (1993). Critical discourse analysis and the marketization of public discourse: The universities. Discourse and Society, 4(2), pp.133-69.

---- (1995). Media discourse. London: Edward Arnold. 
---- (1996). Technologisation of discourse. In Caldas-Coulthard, C.R. \& Coulthard, M. (Eds.), Texts and practices: Readings in Critical Discourse Analysis, London: Routledge.

--- (2002). Critical discourse analysis in transdisciplinary research. In G. Weiss and R. Wodak (Eds.), Interdisciplinarity in CDA. New York: Palgrave Macmillan.

Fairclough, N. and Wodak, R. (1997). Critical discourse analysis. In T. Van Dijk (Ed.), Discourse as social interaction, discourse studies: A multidisciplinary introduction, Vol. 2 (pp. 258-284). London: Sage Publications.

Hall, S. (1982). The determination of news photographs. In S. Cohen and J. Young (Eds.), The manufacture of news. London: Constable.

Halliday, M.A.K. (1978). Language as social semiotic. London: Edward Arnold.

Halliday, M.A.K. \& Mattheissen, C.(2004). An introduction to functional grammar. 3rd edition. London: Edward Arnold.

Halliday, M.A.K. \& Martin, J.R. (1993). Writing science: Literacy and discursive power. London: Routledge.

Hodge, R. \& Kress, G. (1988). Social semiotics. Cambridge: Polity Press.

----- (1993). Language as ideology. 2nd edition. London: Routledge.

Iedema, R. (2003). Multimodality resemiotization: Extending the analysis of discourse as multi-semiotic practice. Visual Communication, 2(1), pp.29-58.

Jewitt, C. \& Oyama, R. (2001). Visual meaning: A Social Semiotic approach. In van Leeuwen and Jewitt (Eds.), Handbook of visual analysis (pp.134-156). London: Sage Publications.

Kress, G. (2003). Literacy in the new media age. London: Routledge.

Knox, J. (2007). Verbal-visual communication on online newspaper home pages. Visual Communication, 6(1), pp.19-53.

Kress, G. and van Leeuwen, T. (1992). Structures of visual representation. Journal of Literary Semantics, 11(2), pp.91-117.

---- (2001). Multimodal discourse: The modes and media of contemporary communication. New York: Oxford University Press.

---- (2002). Colour as a semiotic mode: Notes for a grammar of colour. Visual Communication, 1(3): pp.343-368.

---- (2006). Reading images: The grammar of visual design. 2nd Edition. London: Routledge. 
Lassen, I. (2004). Ideological resources in biotechnology press releases: Patterns of Theme/Rheme and Given/New. In L. Young \& C. Harrison (Eds.), Systemic Functional Linguistics and Critical Discourse Analysis (pp.264-279). New York: Continuum.

Lewis, A.R. (2007). The American culture of war: The history of U.S. military force from World War II to Operation Iraqi Freedom. New York: Routledge.

Lemke, J.L. (1998). Multiplying meaning: Visual and verbal semiotics in scientific text. In J.R. Martin \& R. Veel (Eds.), Reading Science. London: Routledge.

---- (1999). Discourse and organizational dynamics: Website communication and institutional change. Discourse \& Society, 10(1), pp.21-47.

---- (2002). Travels in hypermodality. Visual Communication, 10(1), pp.299-235.

---- (2004). Multiplying intelligences: Hypermedia and social semiotics. In Joe L. Kincheloe, Ed. Multiple Intelligences Reconsidered (pp.177-200). New York: Peter Lang.

---- (2005). Multimedia genres and traversals. Folia Linguistica, 39(1-2), pp.45-56.

Machin, D. (2004). Building the world's visual language: The increasing global importance of image banks in corporate media. Visual Communication, 3(3), 316-36.

Machin, D. \& van Leeuwen, T. (2005). Computer games as political discourse: The case of Black Hawk Down. Journal of Language and Politics, 4(1), pp.119-141.

Martin, J.R. (1997). Analysing genre: Functional parameters. In F. Christie and J.R. Martin (Eds.), Genre and institutions: Social processes in the workplace and school (pp. 3-39). London: Cassell.

---- (2004). Mourning: How we get aligned. Discourse \& Society, 15(2-3), pp.32144.

Martinec, R. \& Salway, A. (2005). A system for image-text relations in new (and old) media. Visual Communication, 4(3), pp.337-71.

Mautner, G. (2005). Time to get wired: Using web-based corpora in critical discourse analysis. Discourse and Society, 16(6), pp.809-828.

O'Toole, M. (1994). The language of displayed art. Rutherford, NJ: Fairleigh Dickinson University Press.

Royce T. \& Bowcher, W. (2007). New directions in the analysis of multimodal Discourse. London: Routledge.

Royce T. (2007). Intersemiotics complimentarity: A framework for multimodal discourse analysis. In T. Royce and W. Bowcher (Eds.), New directions in the analysis of multimodal Discourse (pp.239-274). London: Routledge. 
Sparks, C. (2000). From dead trees to live wires: The Internet's challenge to traditional newspapers. In J. Curran and M. Gurevitch (Eds.), Mass media and society. $3^{\text {rd }}$ Edition (pp.268-92). London: Arnold.

Teo, P. (2004). Ideological dissonances in Singapore's national campaign posters: A semiotic deconstruction. Visual Communication, 3(2), pp.189-212.

Van Dijk, T. (2001). Miltidisciplinary CDA: A plea for diversity. In R. Wodak and M. Meyer (Eds.), Methods of Critical Discourse Analysis (pp.1-13). London: Sage Publications.

van Leeuwen, T. (2003). A multimodal perspective on composition. In T. Ensink \& C. Sauer (Eds.), Framing and perspectivising in discourse (pp.23-61). Amsterdam: John Benjamins.

----- (2005). Introducing social semiotics. London: Routledge.

van Leeuwen, T. \& Jaworski, A. (2002). The discourses of war photography: Photojournalistic representations of the Palestinian-Israeli war. Journal of Language and Politics, 1(2), pp.255-76.

van Leuuwen, T. \& Jewitt, C. (2001). Handbook of visual analysis. London: Sage Publications.

White, P.R.R. (2003). News as history: Your daily gossip. In J.R. Martin and R. Wodak (eds.) Re/reading the past: Critical and functional perspectives on time and value. (pp.61-89). Amsterdam: John Benjamins.

Wodak, R. (2001). What CDA is about - a summary of its history, important concepts and its developments. In Wodak R. and M. Meyer (Eds.), Methods of Critical Discourse Analysis (pp.1-13). London: Sage Publications.

Young, L. and Fitzgerald, B. (2006). Language and power: How discourse influences society. London: Equinox. 


\section{Table of Contents}

Section I - Coalition Vehicles - p. 1-2

(1) CNN - Carrier (USS Carl Vinson)

(2) BBC - Carrier (USS Harry S. Truman)

(3) CNN - Destroyer (USS Arleigh Burke)

(4) BBC - Destroyer (USS Arleigh Burke)

(5) $\mathrm{CNN}$ - Submarine

(6) BBC - Submarine

(7) $\mathrm{BBC}-$ Challenger Tank (UK)

(8) BBC - Abrams Tank (US)

(9) $\mathrm{CNN}-\mathrm{F}-16$ Fighter (US)

(10) BBC - F-16 Fighter (US)

(11) CNN - Tornado (UK)

(12) BBC - Tornado (UK)

(13) CNN - B-52 Bomber (US)

(14) BBC - B-52 Bomber (US)

Section II - Coalition Troops - p. 3

(1) $\mathrm{CNN}$ - US Troops

(2) $\mathrm{BBC}-4^{\text {th }}$ Infantry Division (US)

(3) $\mathrm{BBC}-101^{\text {st }}$ Airborne Division (US)

(4) BBC - 3 Commando Royal Marines (UK)

(5) BBC - Kurdish Peshmergas

(6) BBC - 33 Engineer Regiment (UK)

(7) BBC-Australian Special Forces

(8) BBC - US Special Forces - Movement
Section III - Iraqi Troops and Equipment p. 4
(1) CNN - Iraq's Army
(2) BBC - Iraq's Army
(3) CNN - Republican Guard
(4) $\mathrm{CNN}$ - Iraqi Equipment
(5) BBC - Lion of Babylon Tank
(6) BBC - Lion of Babylon Tank
(7) CNN - Iraq's Air Force (MiG-23 Fighter)
(8) BBC - Iraq's Air Force (Mirage F-1 Fighter)

Section IV - Coalition Weapons - p. 5

(1) CNN - Tomahawk Cruise Missile (US)

(2) BBC - Storm Shadow Cruise Missile (UK)

(3) CNN - JDAM Bomb (US)

(4) BBC - JDAM Bomb (US)

(5) CNN - AMRAAM Missile (US)

(6) BBC - Storm Shadow Cruise Missile (UK)

(7) BBC - Smart Bombs (Introduction)

(8) BBC - "Not So Smart"

\section{Section V - Biological and Chemical}

Weapons - p. 6

(1) CNN - "Hidden Arsenal"

(2) $\mathrm{BBC}-\mathrm{Bio} / \mathrm{Chem}$ Weapons - Introduction

(3) $\mathrm{BBC}$ - Plague

(4) BBC - Nerve Agents 


\section{Section I - Coalition Vehicles}

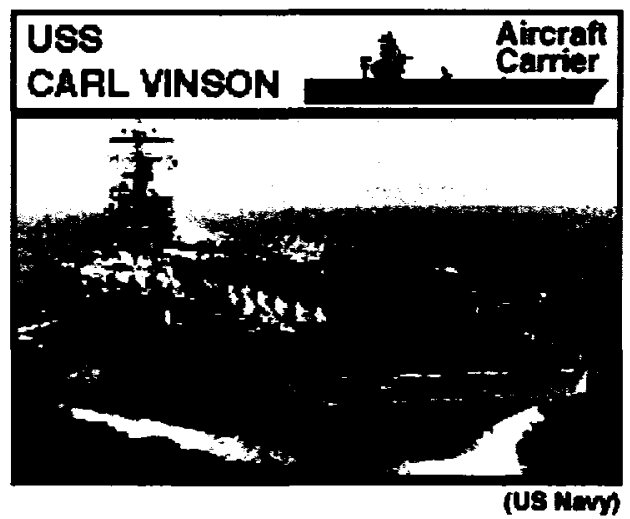

(1) $\mathrm{CNN}$

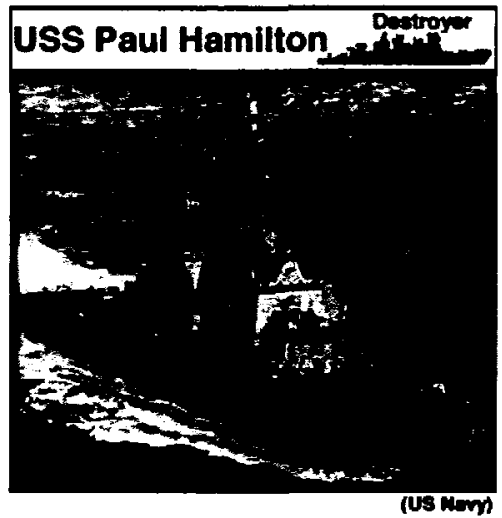

(3) $\mathrm{CNN}$

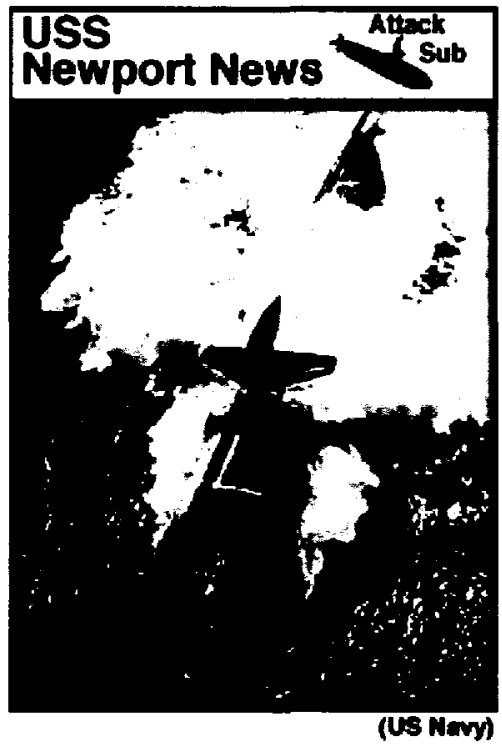

(5) $\mathrm{CNN}$

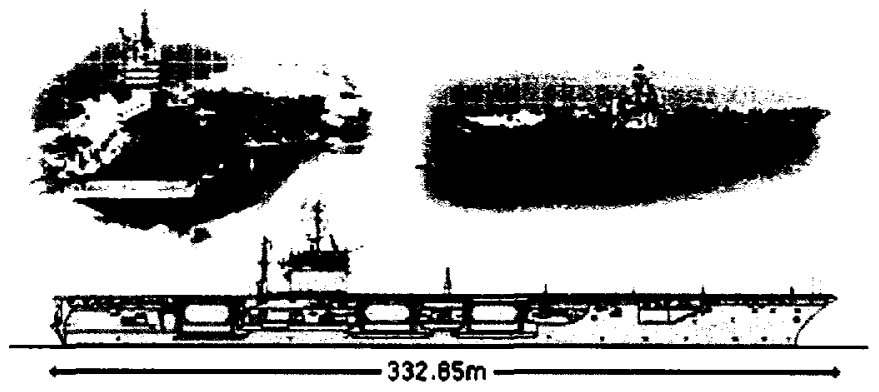

(2) BBC

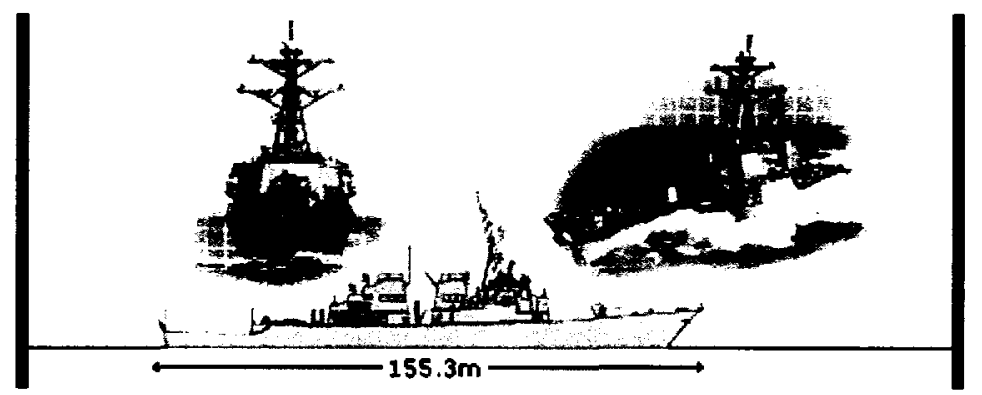

(4) BBC

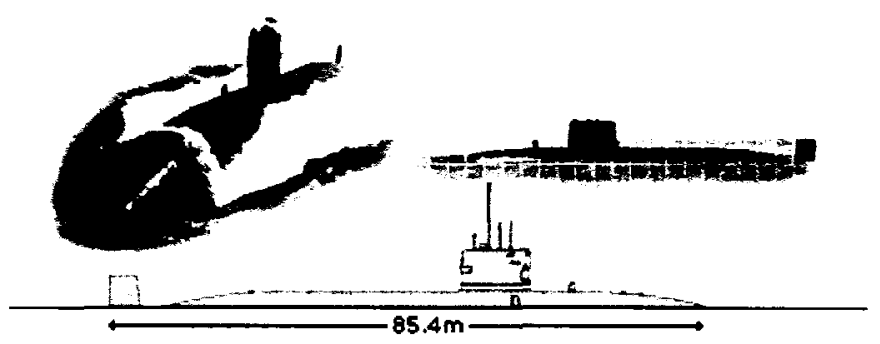

(6) BBC 


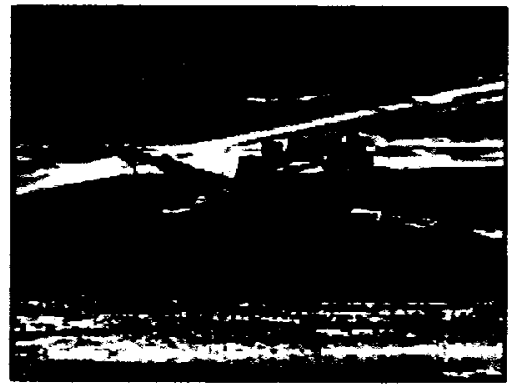

(7) $\mathrm{BBC}$

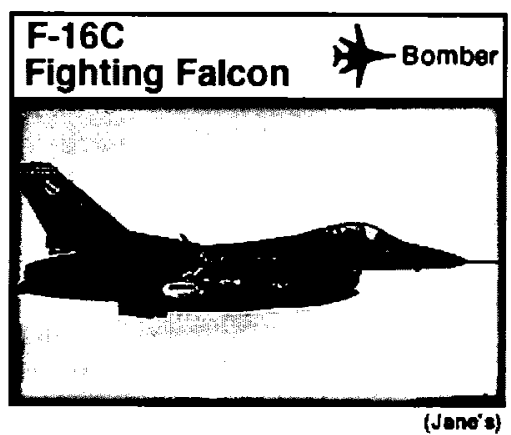

(9) $\mathrm{CNN}$
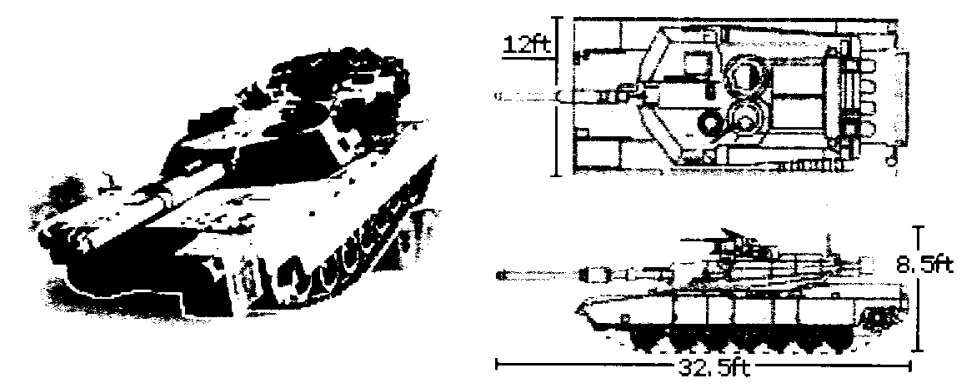

(8) BBC

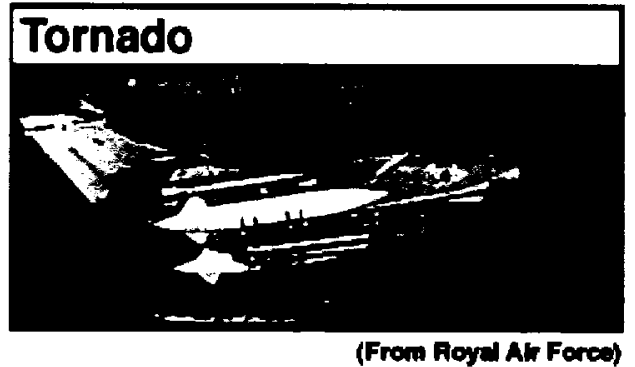

(11) $\mathrm{CNN}$

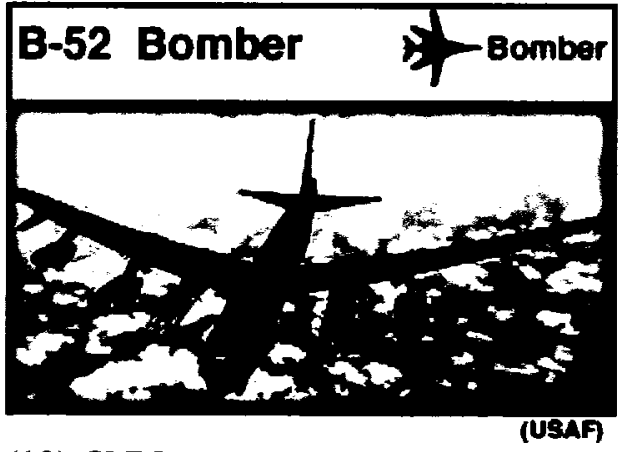

(13) $\mathrm{CNN}$

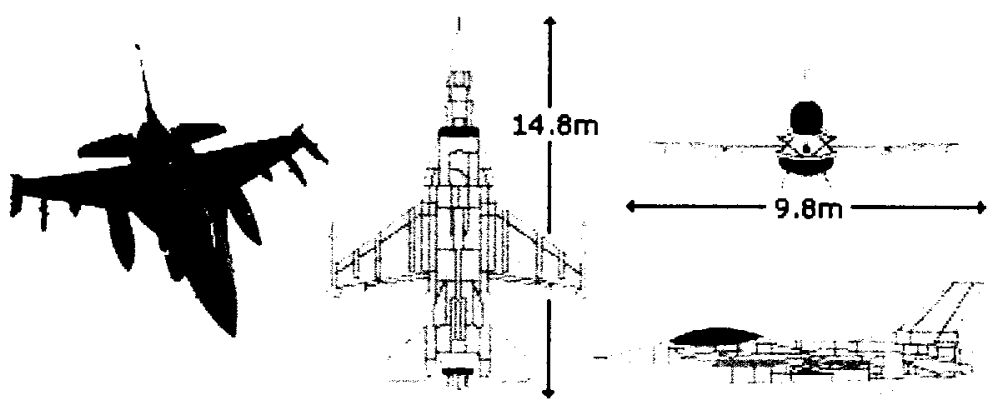

(10) BBC

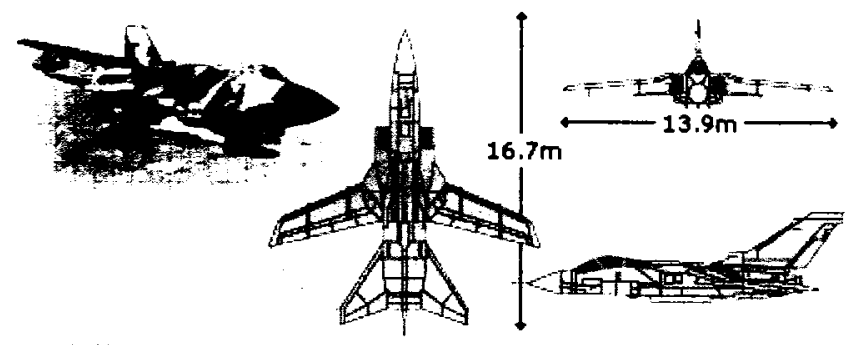

(12) BBC

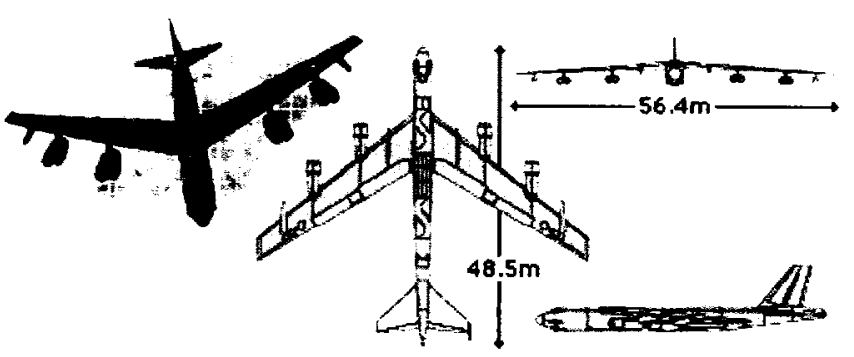

(14) BBC 


\section{Section II - Coalition Troops}

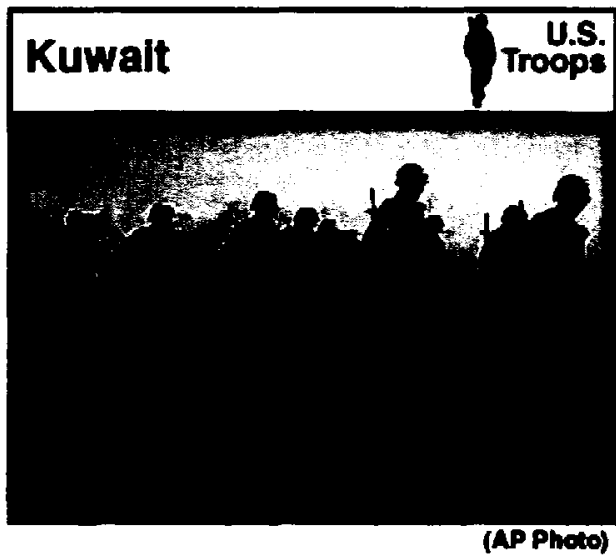

(1) $\mathrm{CNN}$

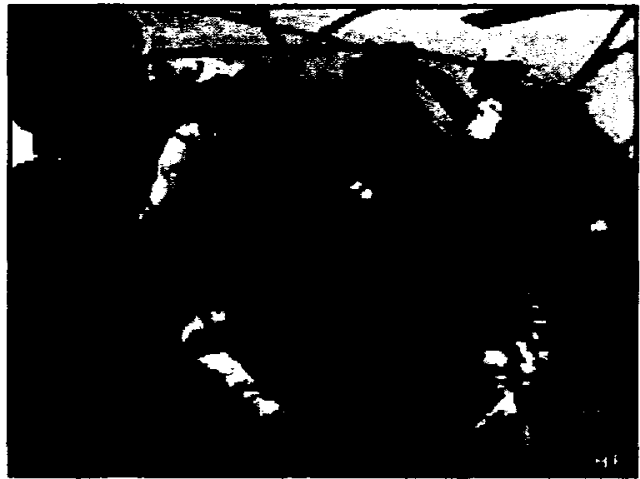

(3) $\mathrm{BBC}$

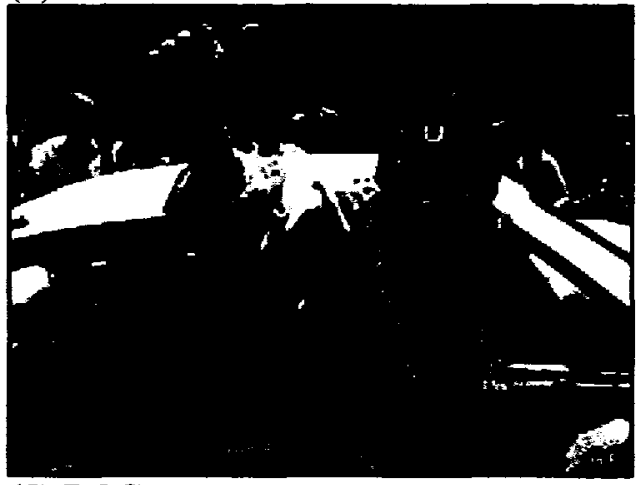

(5) $\mathrm{BBC}$

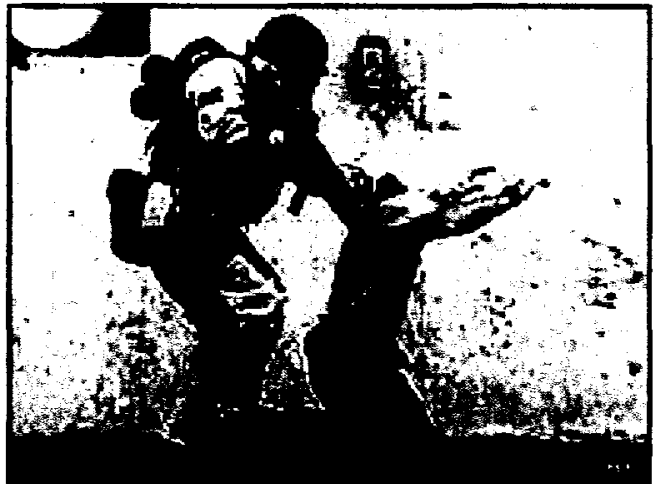

(7) $\mathrm{BBC}$

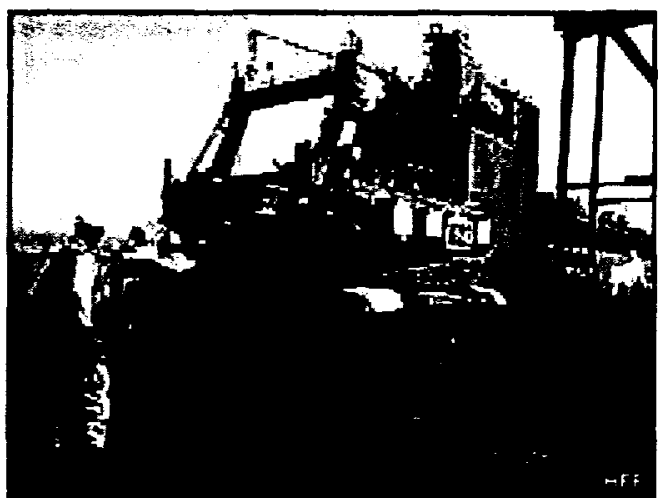

(2) BBC

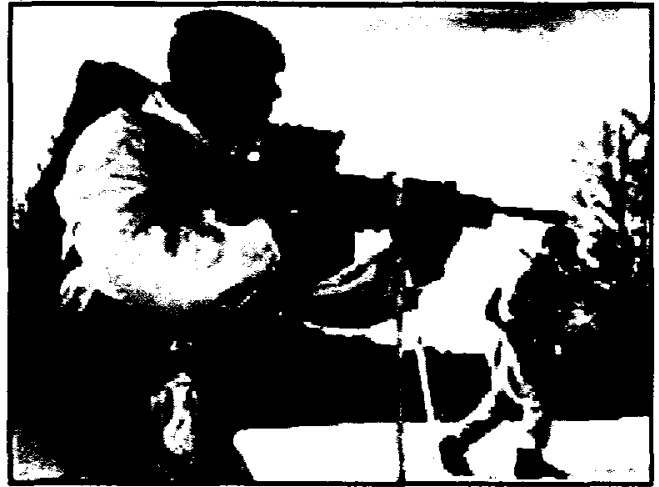

(4) BBC

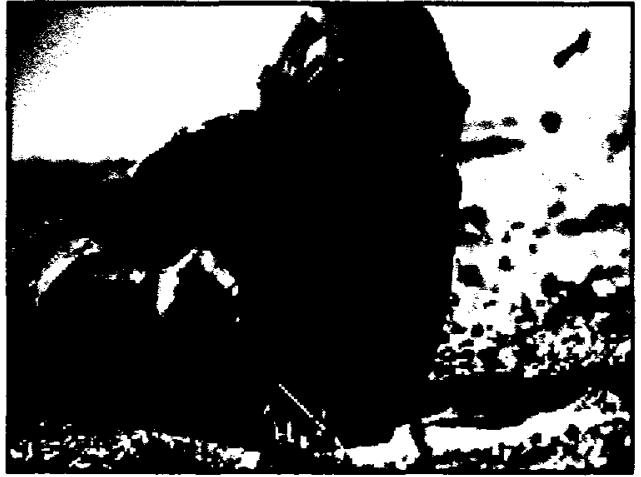

(6) $\mathrm{BBC}$

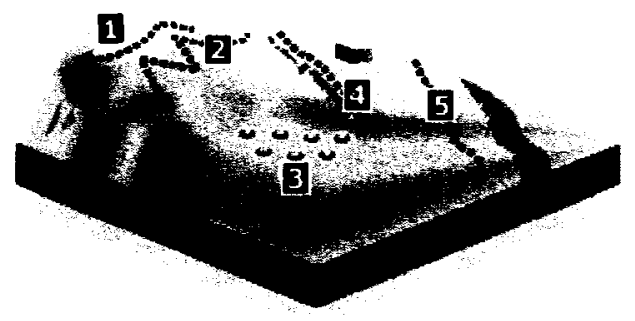

(8) BBC 


\section{Section III - Iraqi Troops and Equipment}

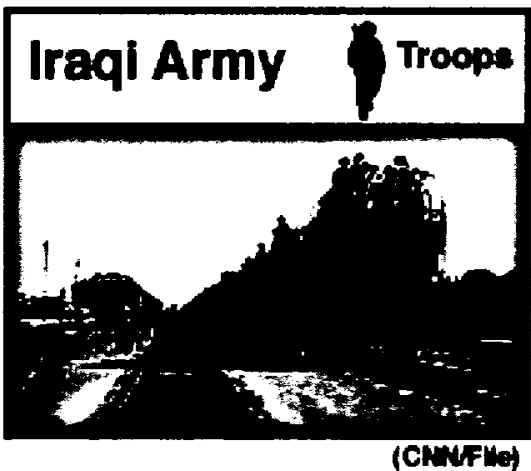

(1) $\mathrm{CNN}$

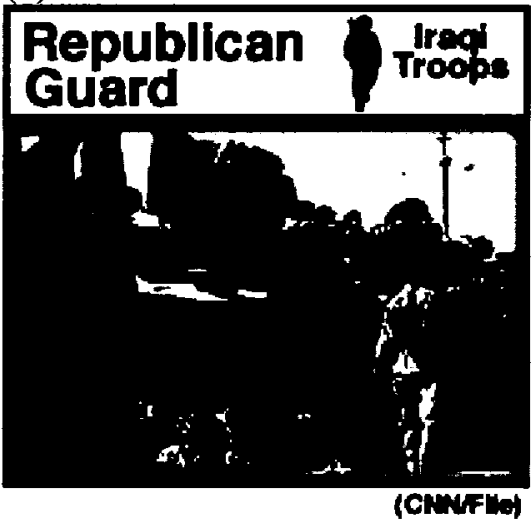

(3) $\mathrm{CNN}$

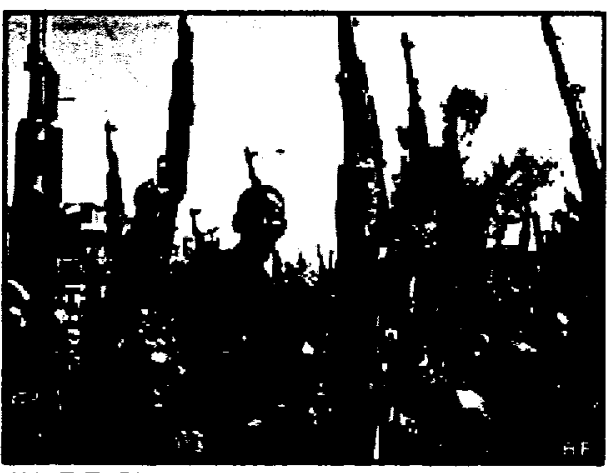

(2) BBC)

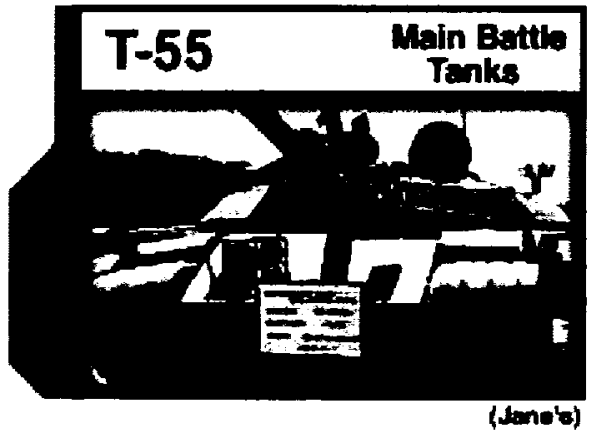

(4) $\mathrm{CNN}$

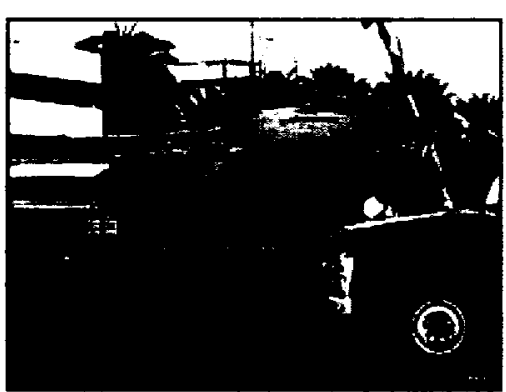

(5) $\mathrm{BBC}$

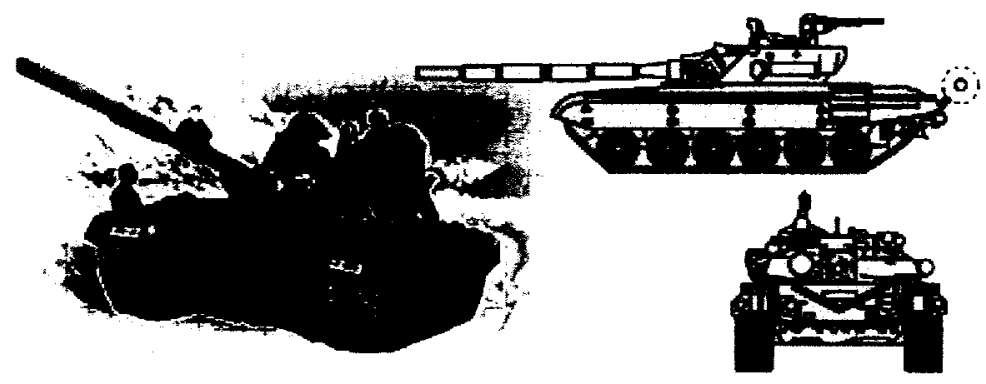

(6) BBC

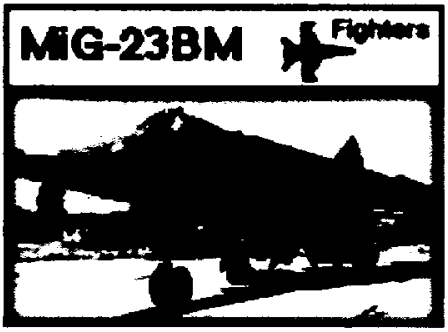

(7) $\mathrm{CNN}$

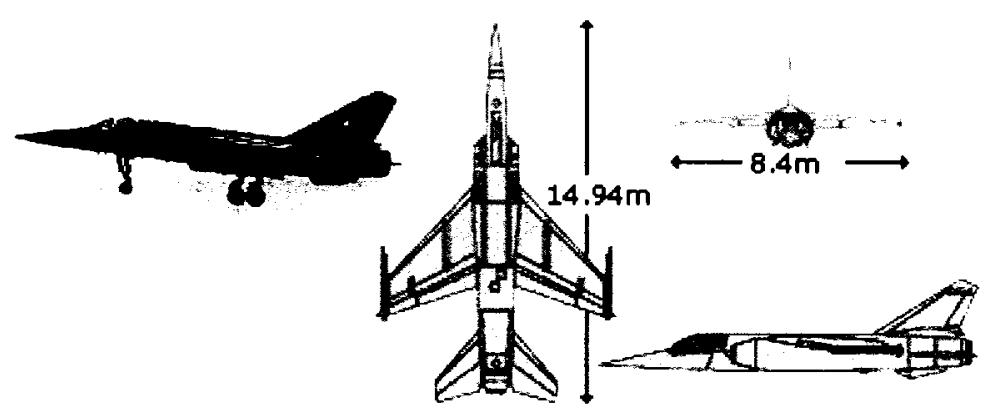

(8) BBC 


\section{Section IV - Coalition Weapons}

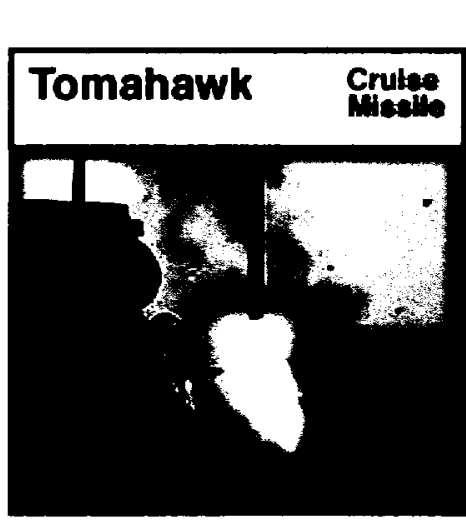

(US Amy)

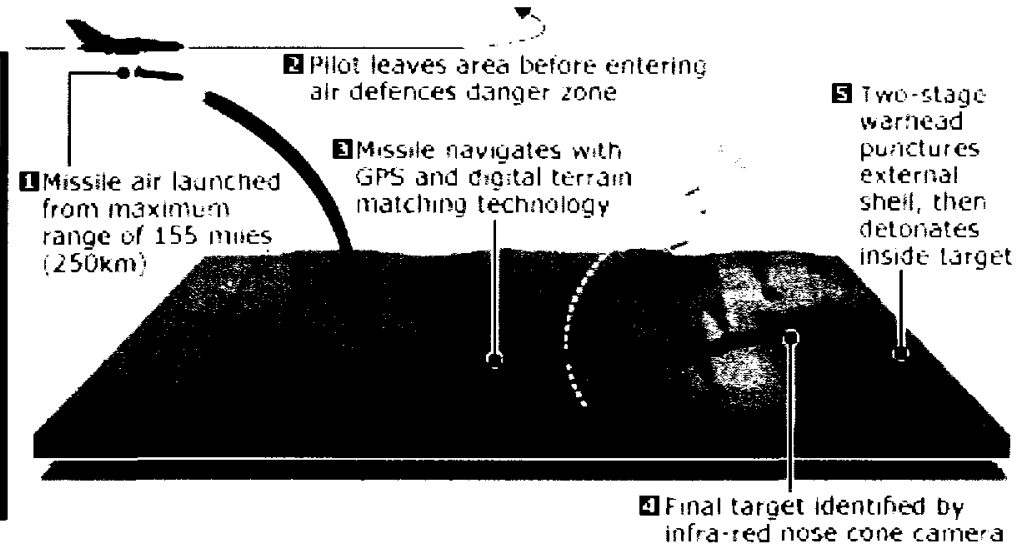

(2) BBC

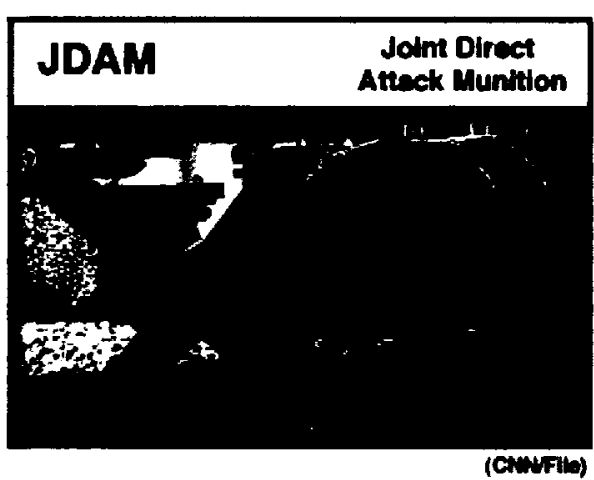

(3) $\mathrm{CNN}$

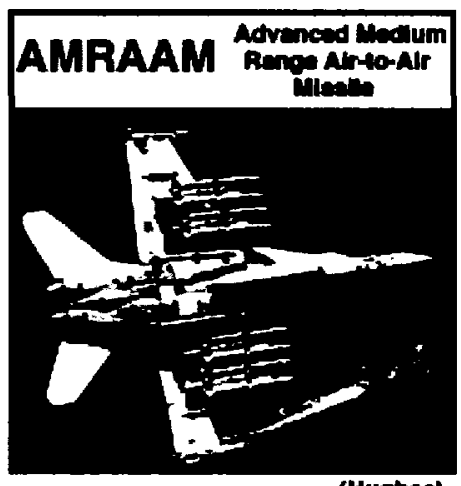

(5) $\mathrm{CNN}$

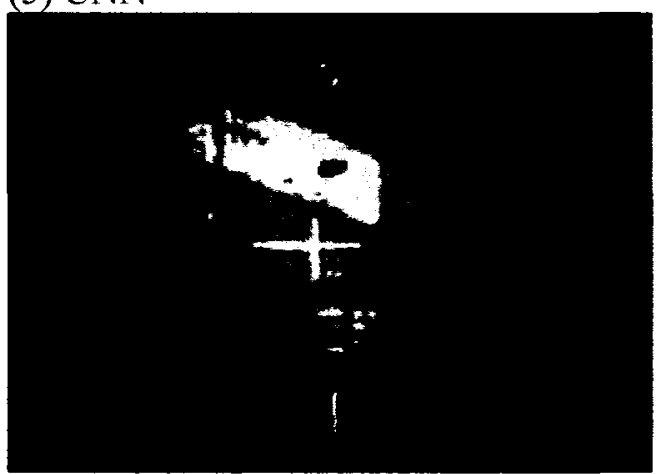

(7) $\mathrm{BBC}$

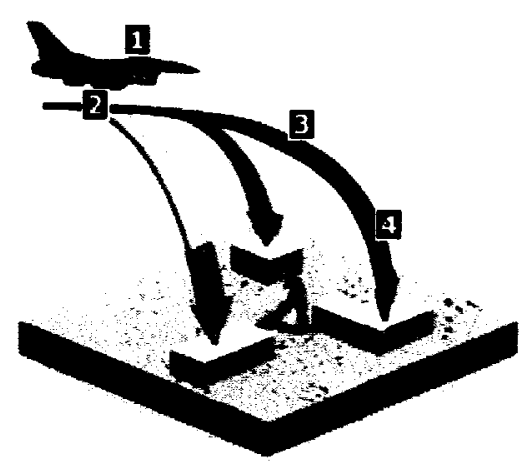

(4) $\mathrm{BBC}$

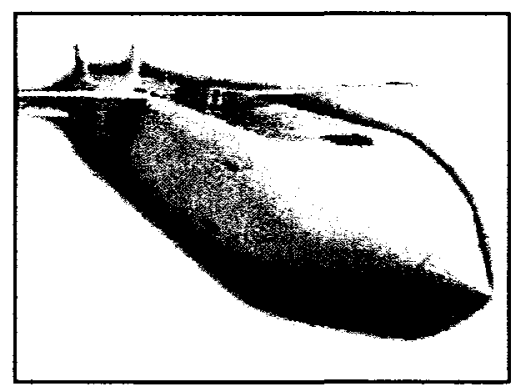

(6) $\mathrm{BBC}$ )

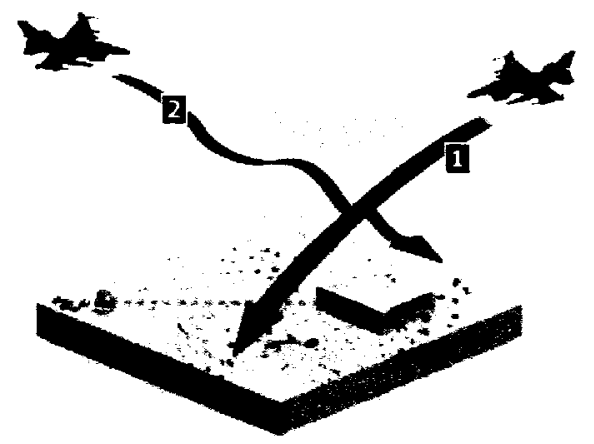

(8) $\mathrm{BBC}$ 
Section V - Biological and Chemical Weapons

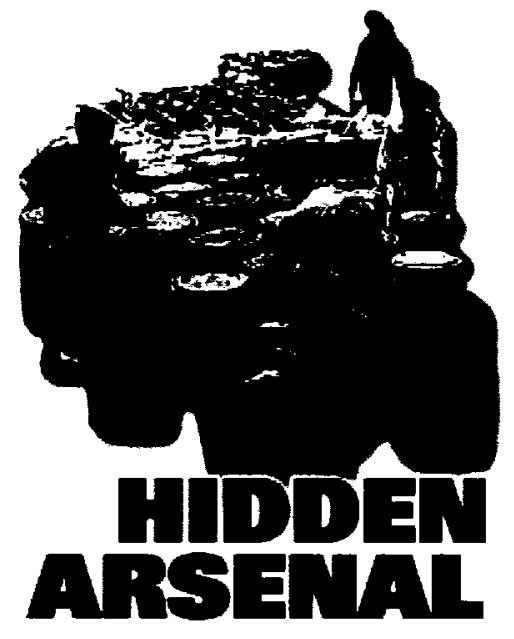

(1) $\mathrm{CNN}$

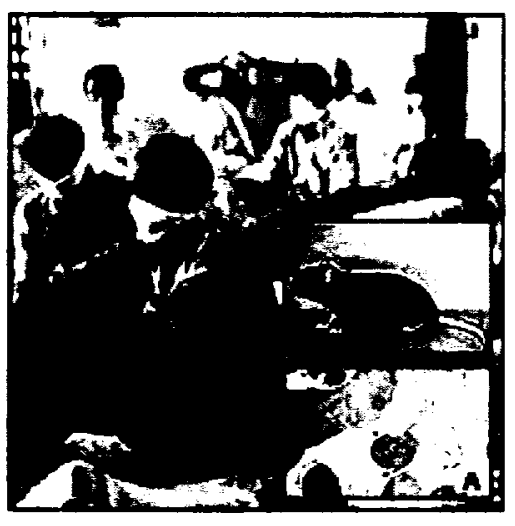

(3) BBC

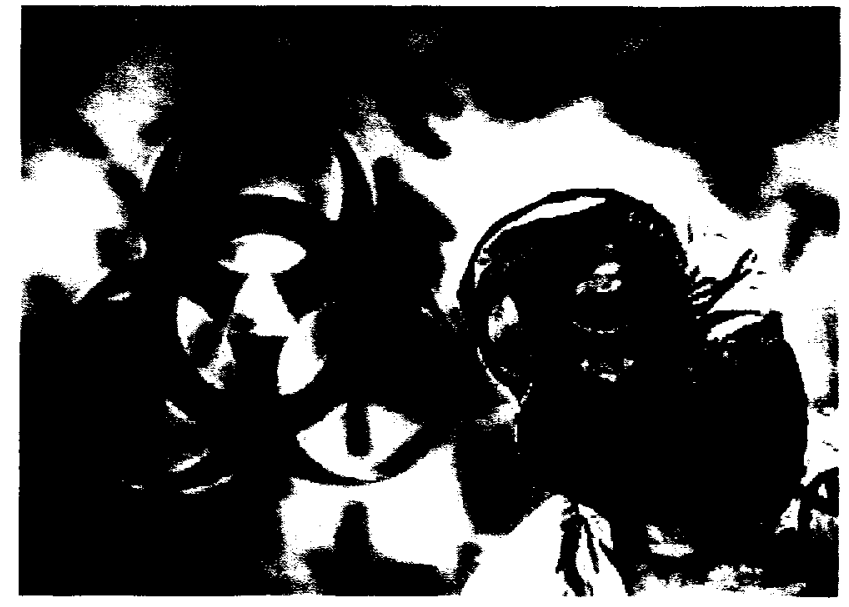

(2) $\mathrm{BBC}$

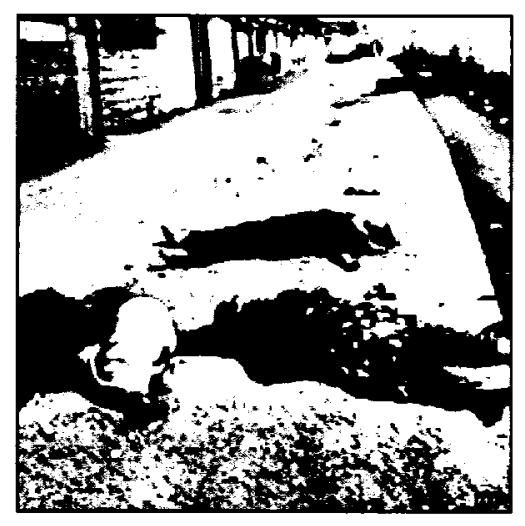

(4) BBC 


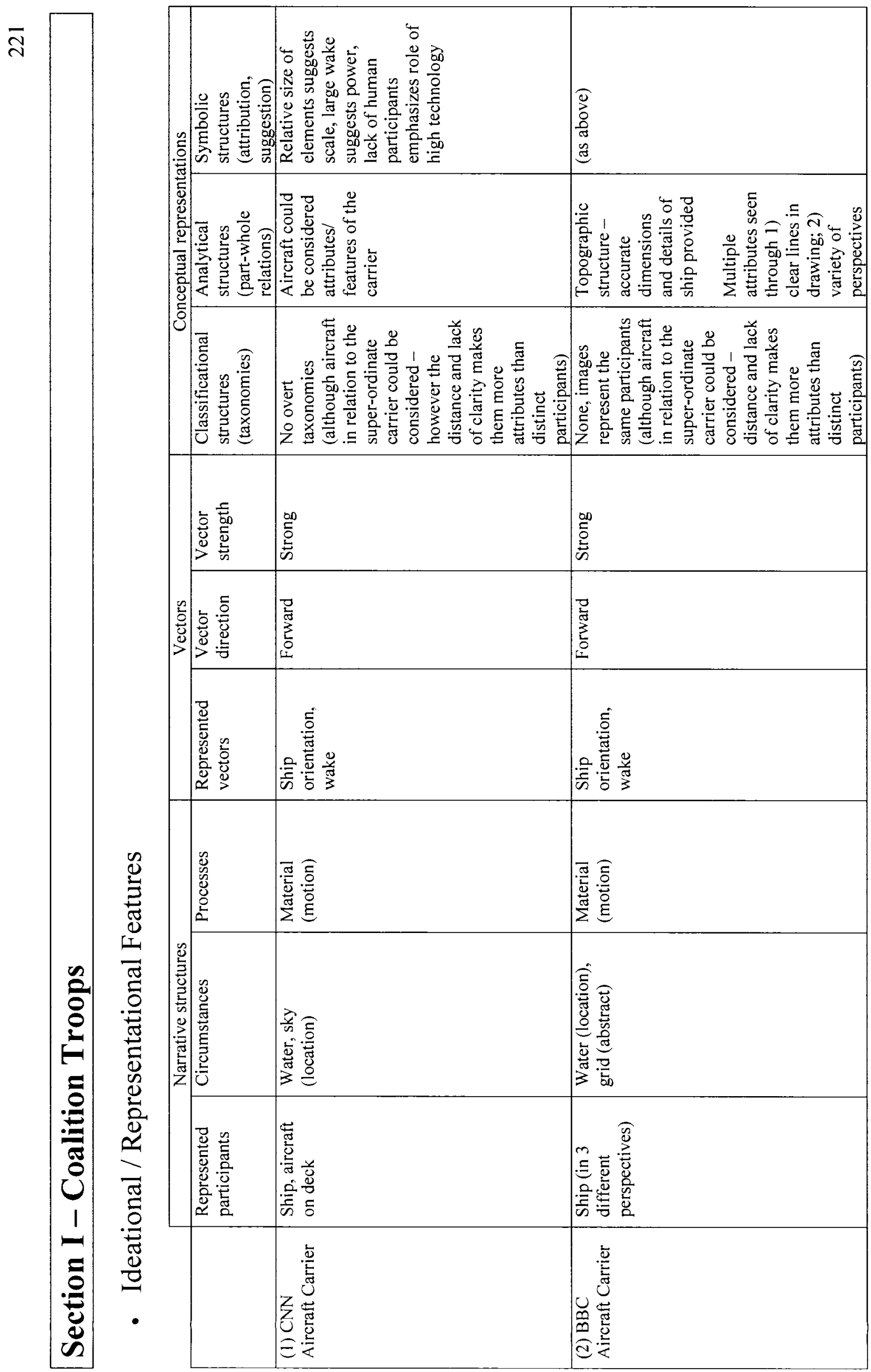




\begin{tabular}{|c|c|c|c|}
\hline 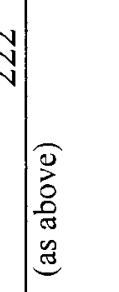 & 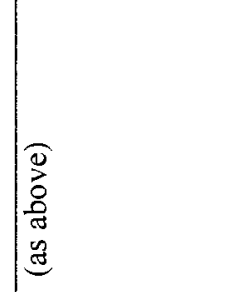 & 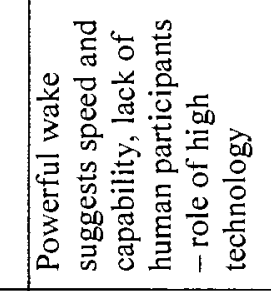 & 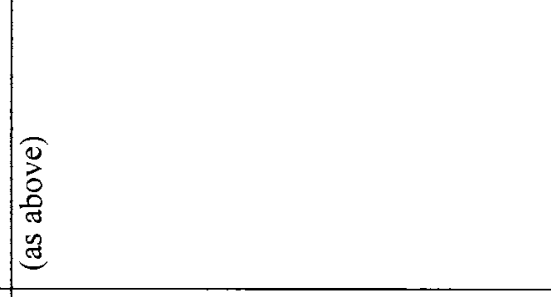 \\
\hline 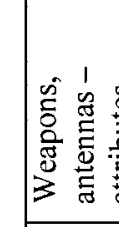 & 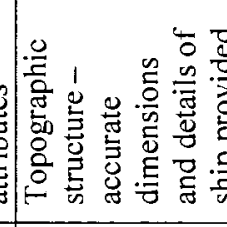 & 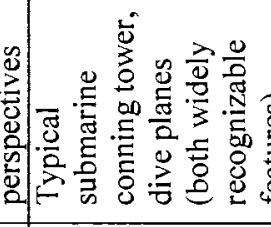 & 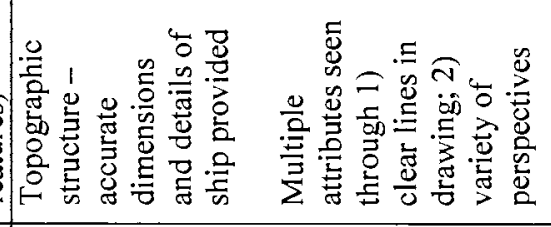 \\
\hline 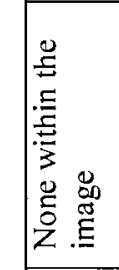 & 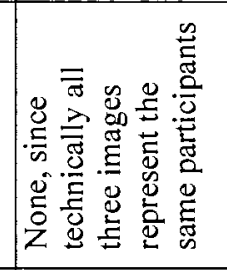 & 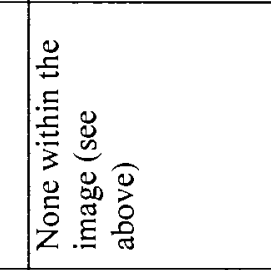 & 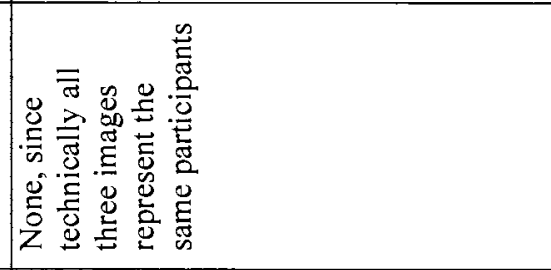 \\
\hline 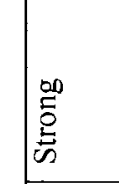 & 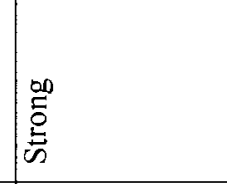 & 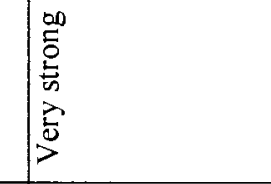 & 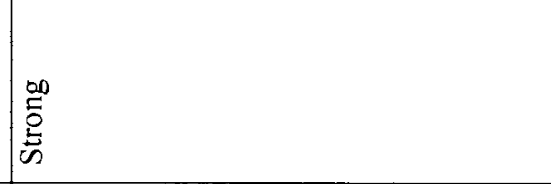 \\
\hline 总 & 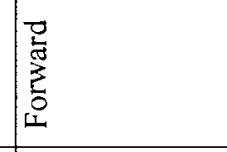 & 总 & 总 \\
\hline 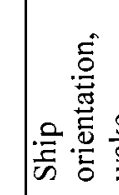 & 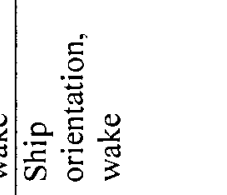 & 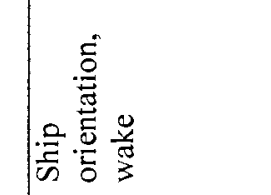 & 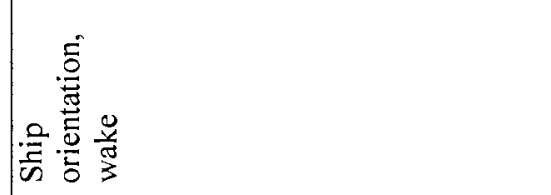 \\
\hline 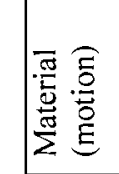 & 亭 & 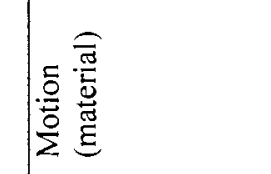 & 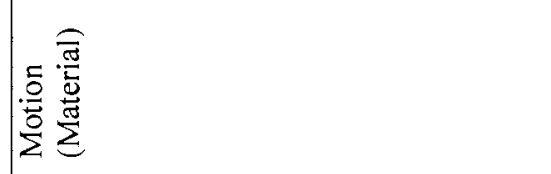 \\
\hline 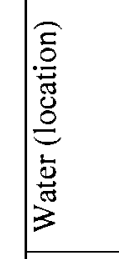 & 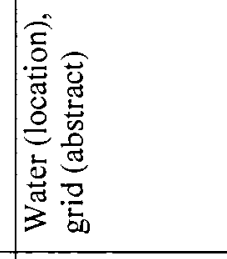 & 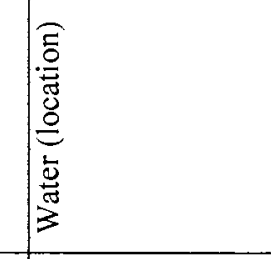 & 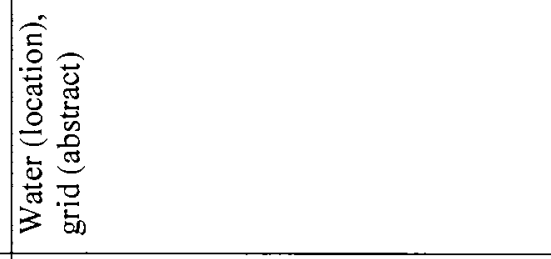 \\
\hline 产 & 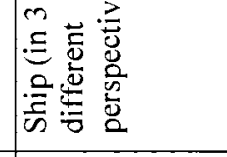 & $\frac{\frac{\partial}{\bar{m}}}{\mathrm{~m}}$ & 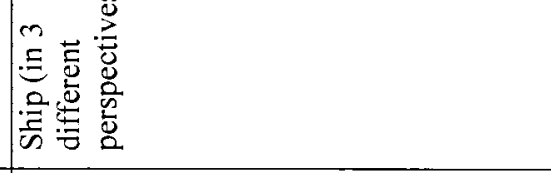 \\
\hline 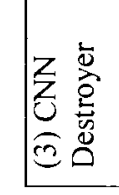 & 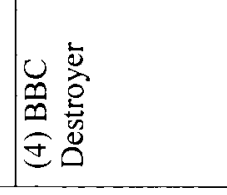 & 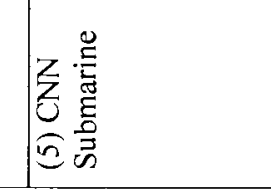 & 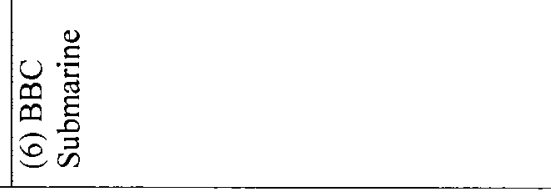 \\
\hline
\end{tabular}




\begin{tabular}{|c|c|}
\hline 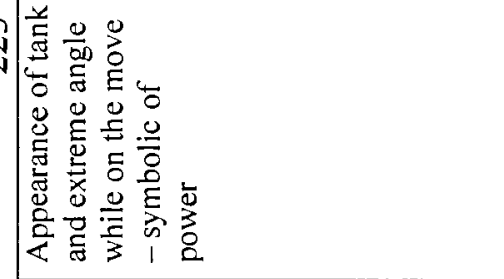 & 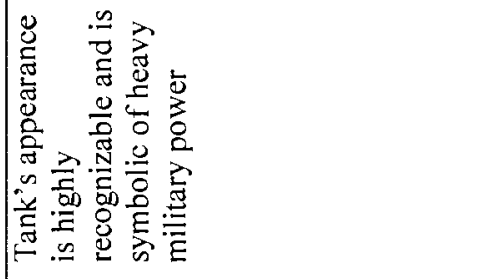 \\
\hline 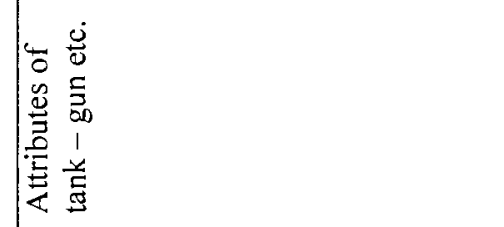 & 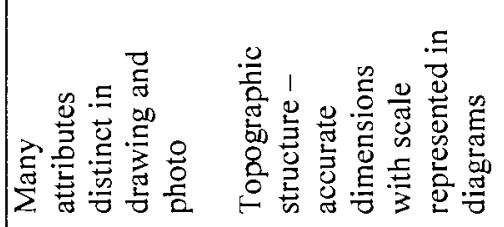 \\
\hline. & 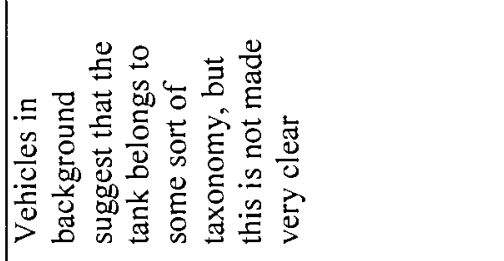 \\
\hline 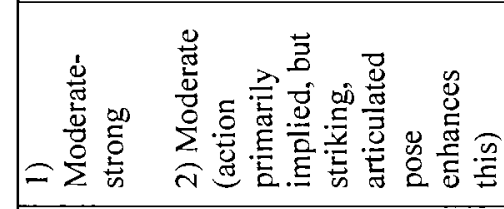 & 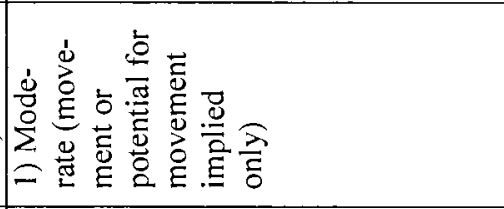 \\
\hline 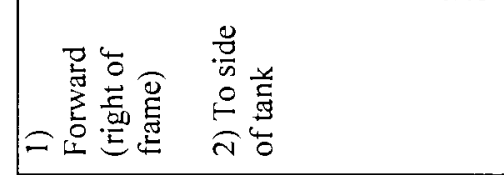 & 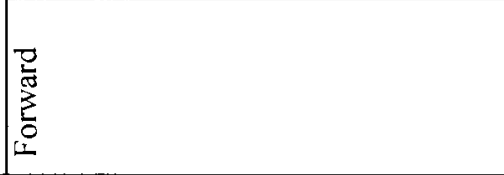 \\
\hline 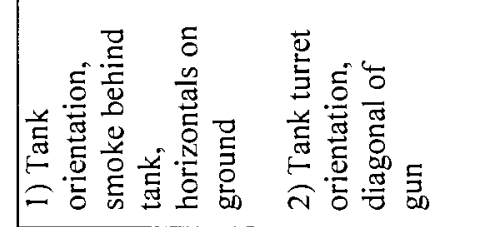 & 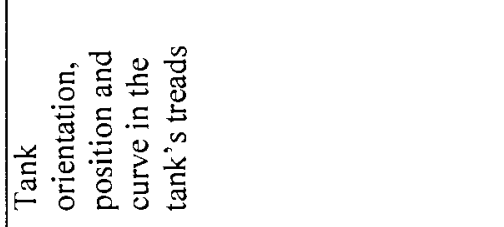 \\
\hline ) & 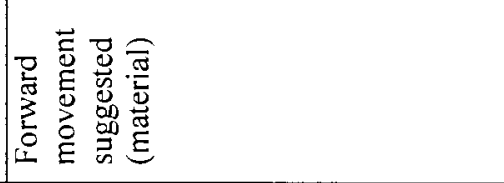 \\
\hline 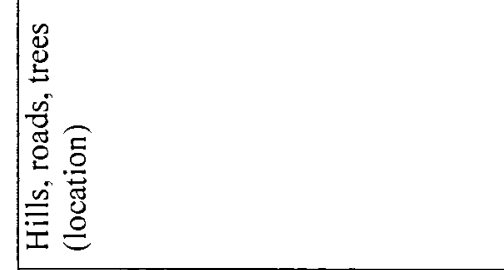 & 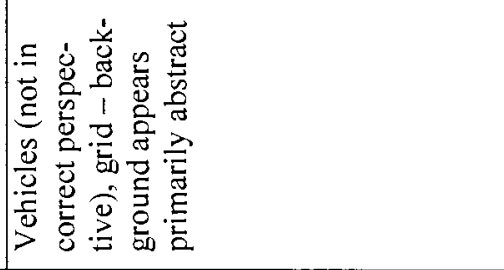 \\
\hline 点 & 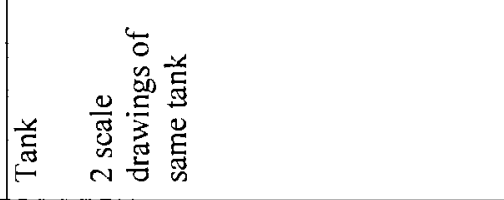 \\
\hline 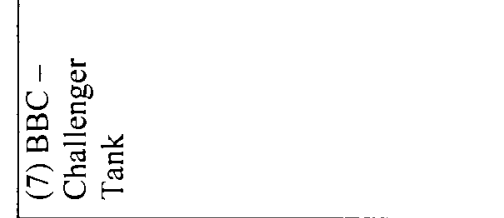 & 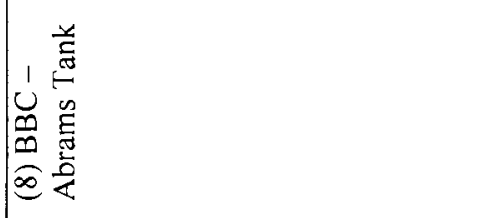 \\
\hline
\end{tabular}




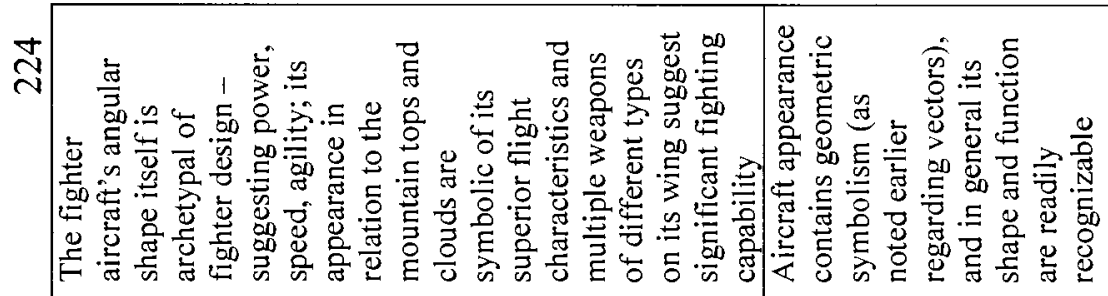

\begin{tabular}{|c|c|}
\hline 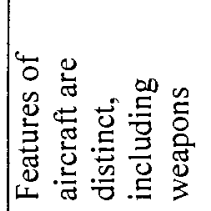 & 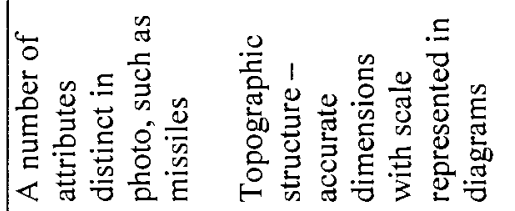 \\
\hline
\end{tabular}

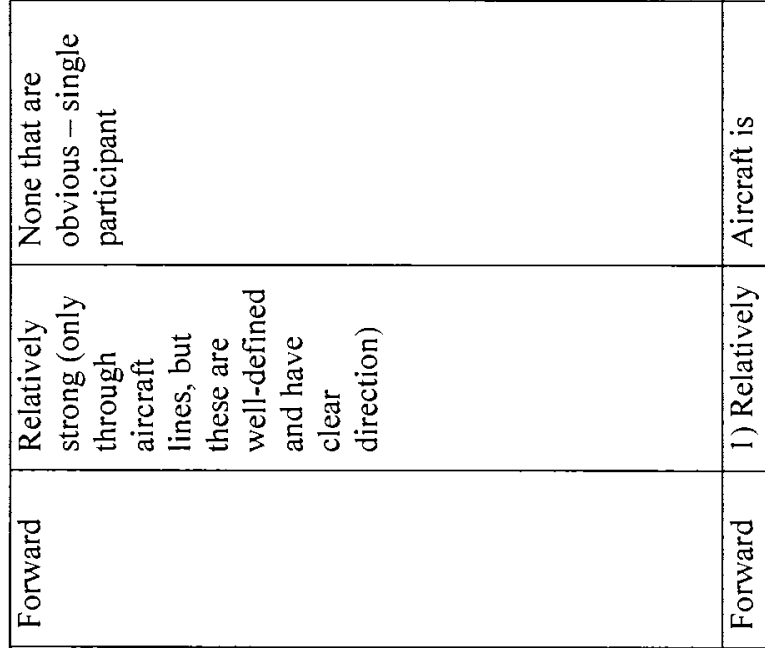

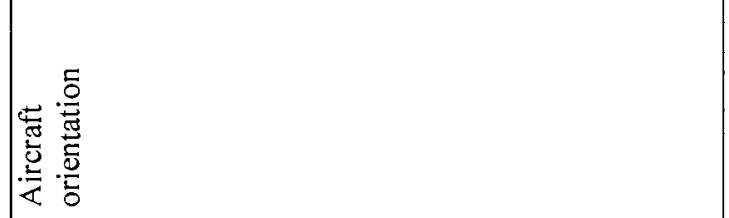

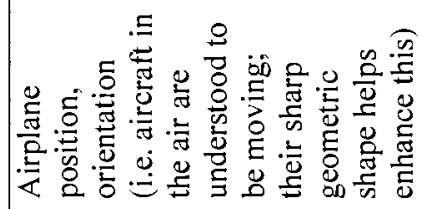

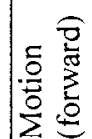

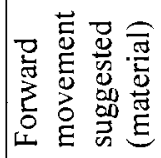

so

응

웅

言高高

站 点

6

$\frac{1}{1}$

造

遂

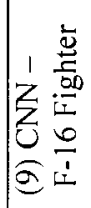

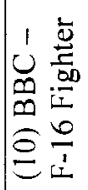




\begin{tabular}{|c|c|c|}
\hline 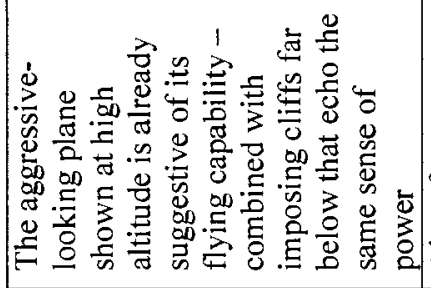 & 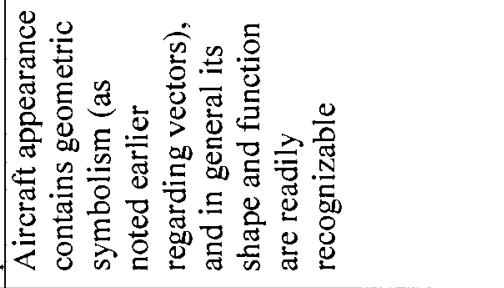 & 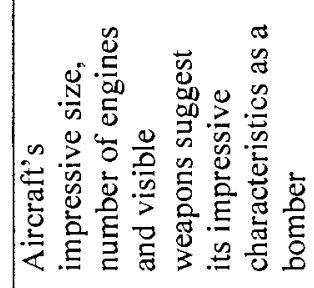 \\
\hline 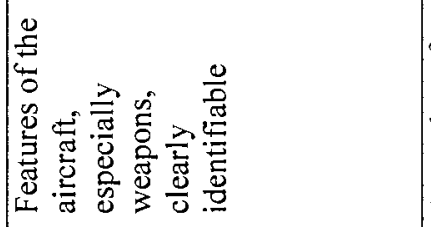 & 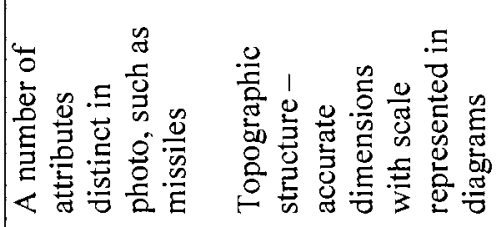 & 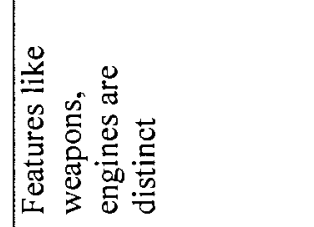 \\
\hline 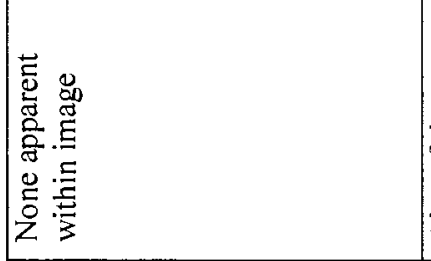 & 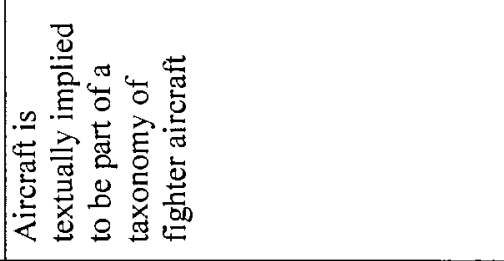 & 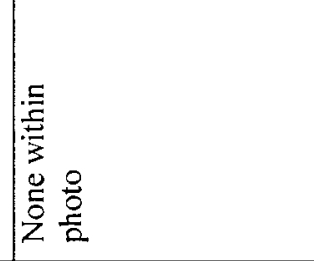 \\
\hline 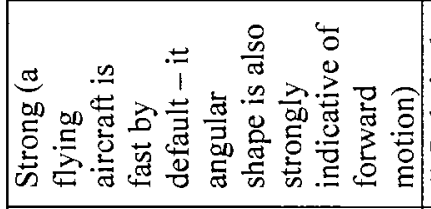 & 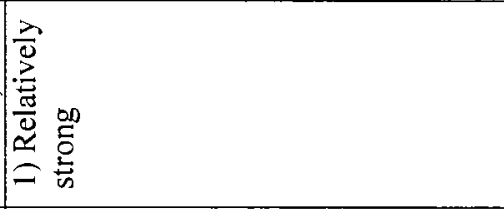 & | \\
\hline 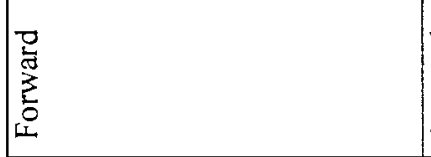 & 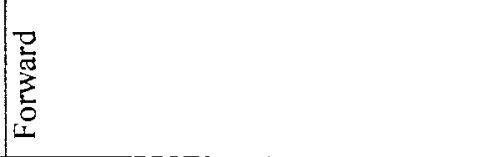 & 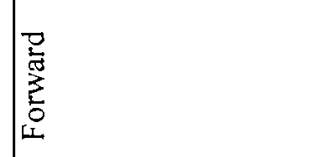 \\
\hline 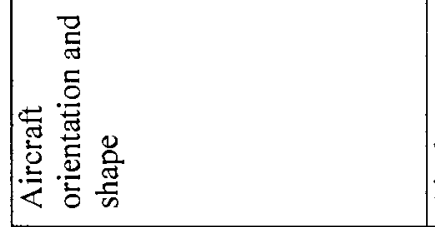 & 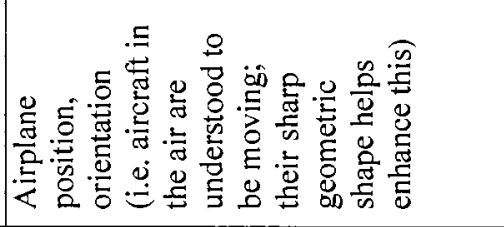 & 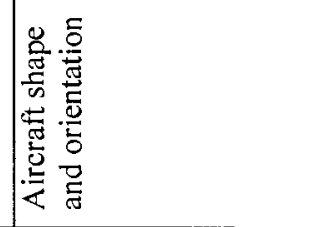 \\
\hline 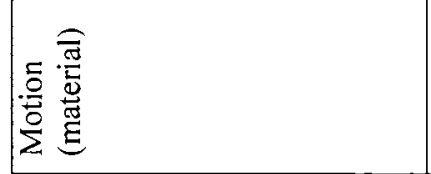 & 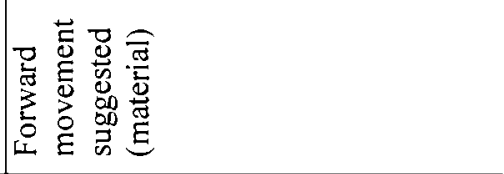 & 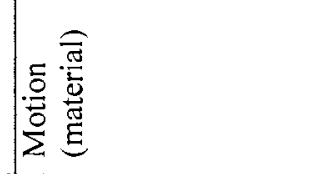 \\
\hline 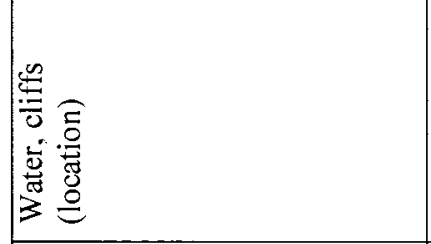 & 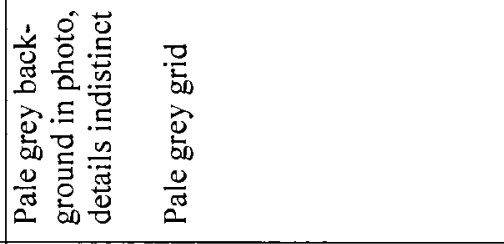 & 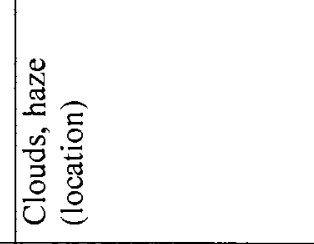 \\
\hline 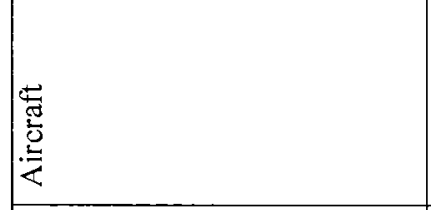 & 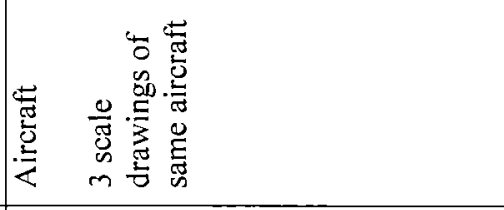 & 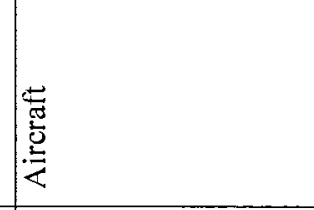 \\
\hline 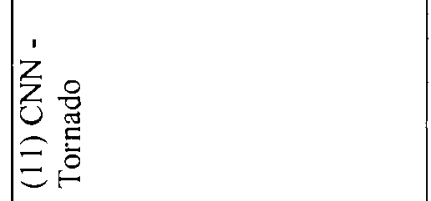 & 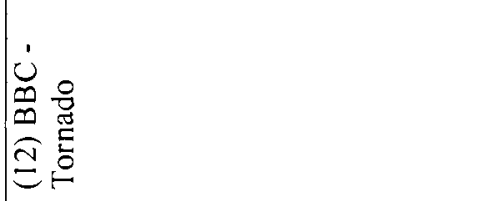 & 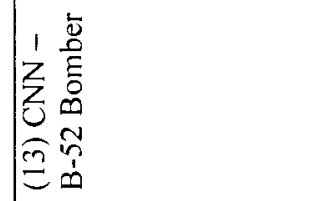 \\
\hline
\end{tabular}




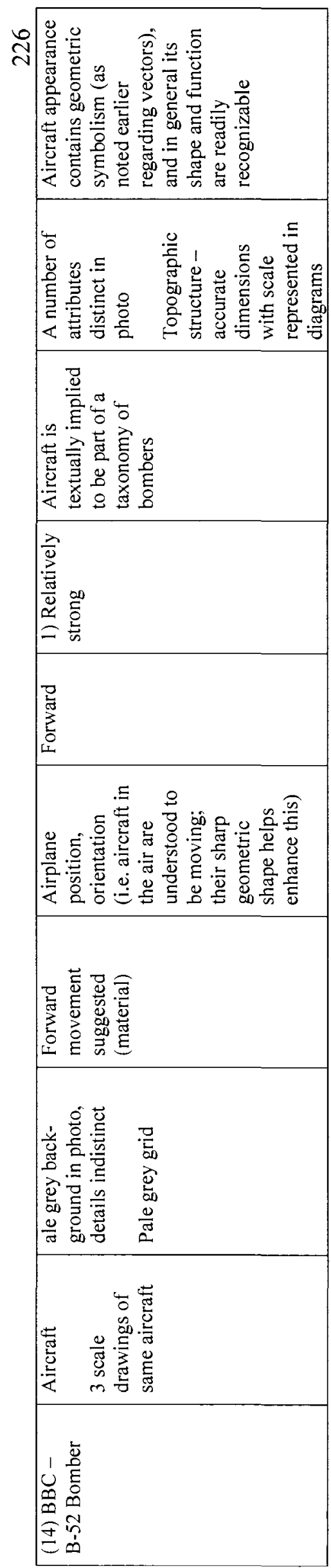


สิ

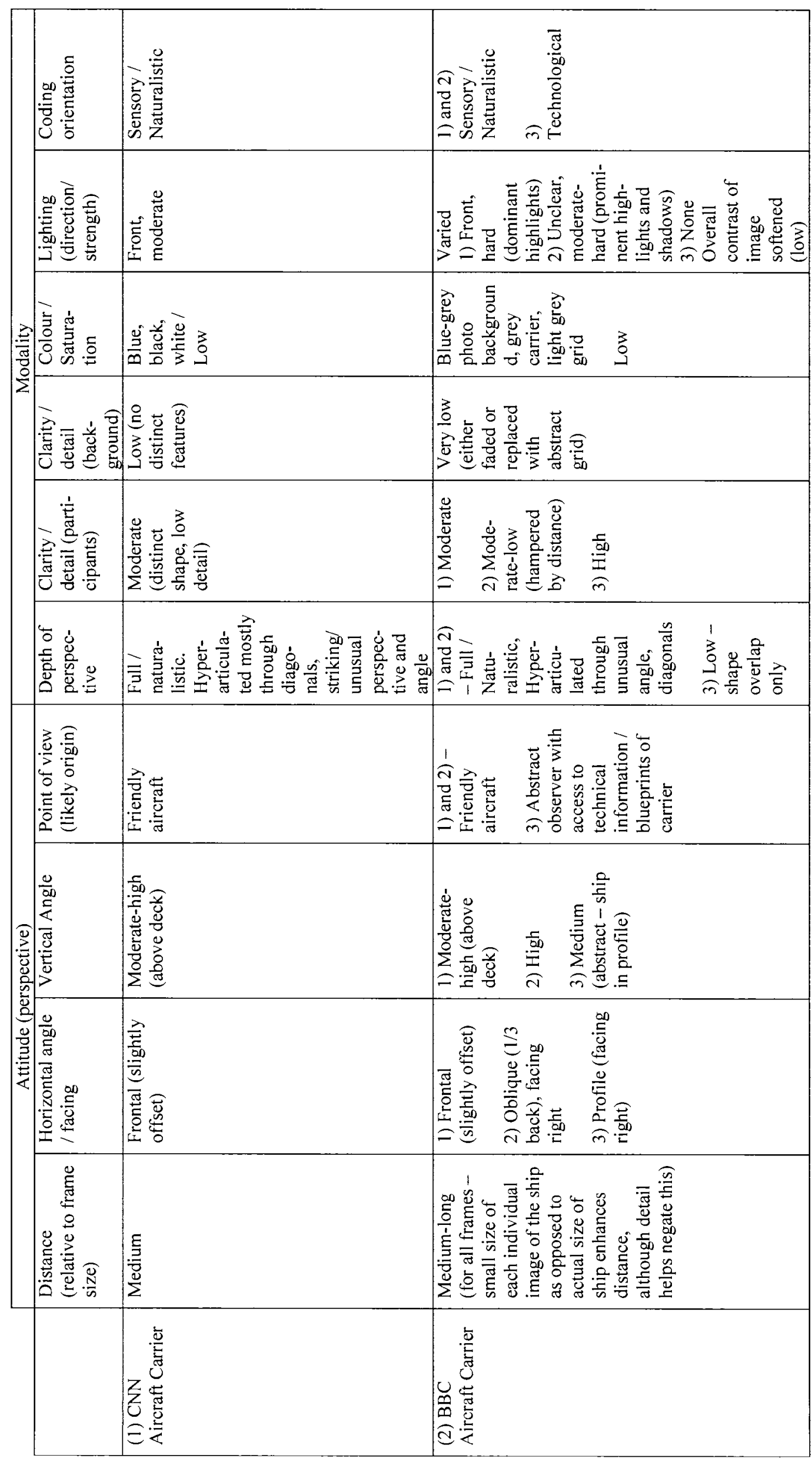




\begin{tabular}{|c|c|c|}
\hline 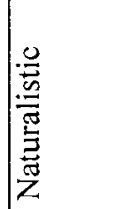 & 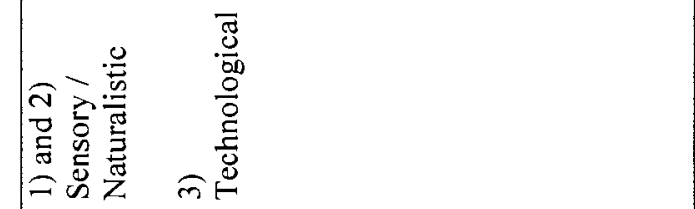 & 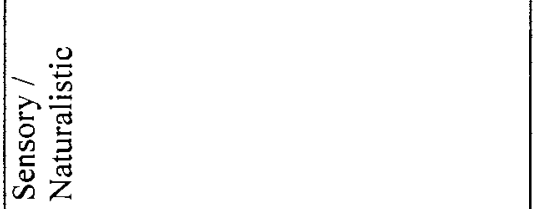 \\
\hline 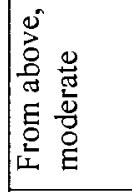 & 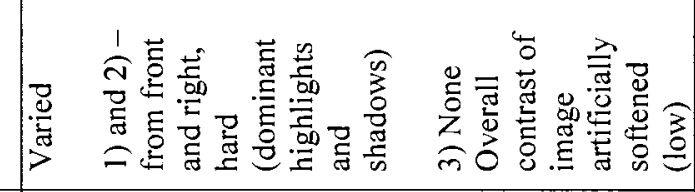 & 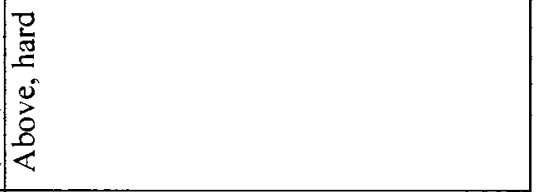 \\
\hline 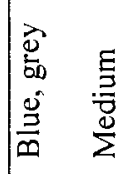 & 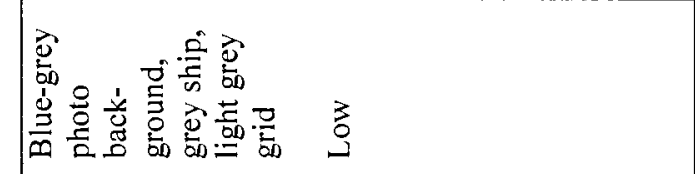 & 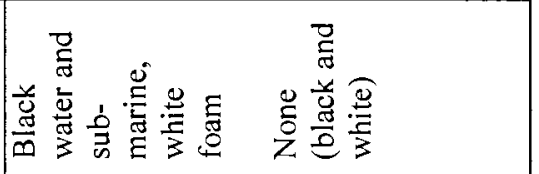 \\
\hline 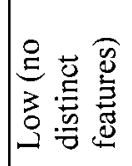 & 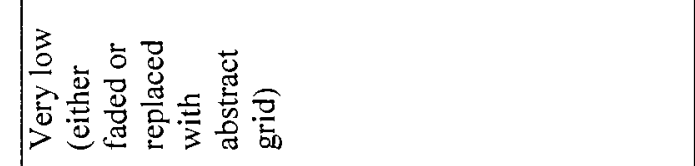 & 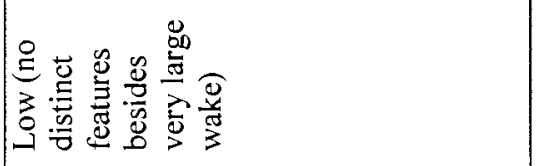 \\
\hline 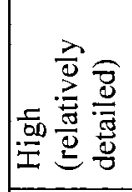 & 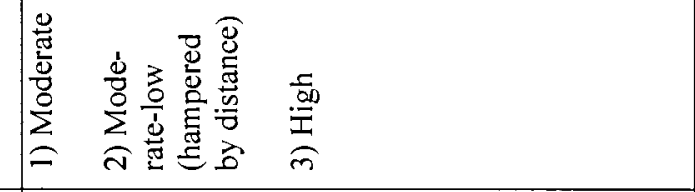 & 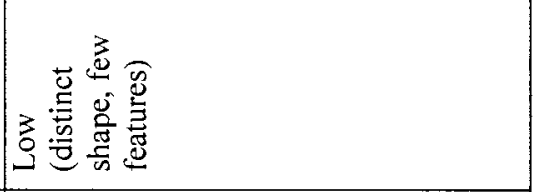 \\
\hline 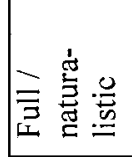 & 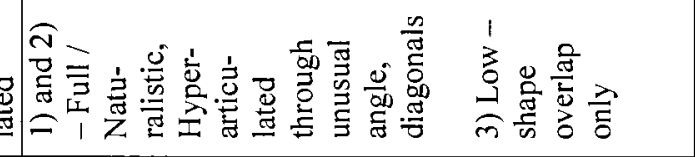 & 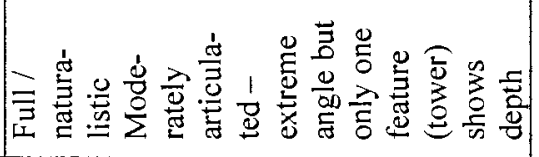 \\
\hline 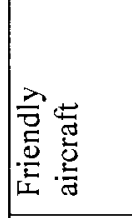 & 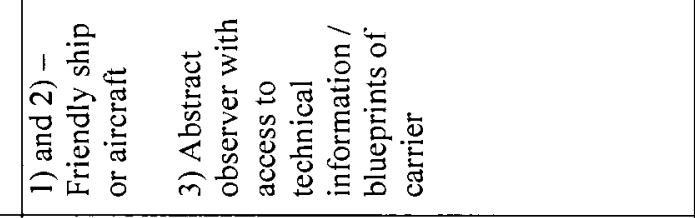 & 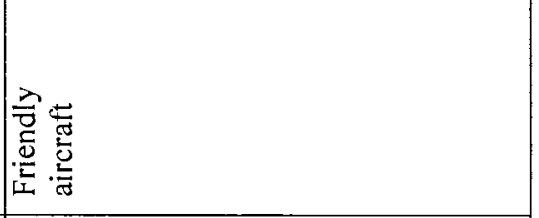 \\
\hline $\mid \frac{5}{.50}$ & 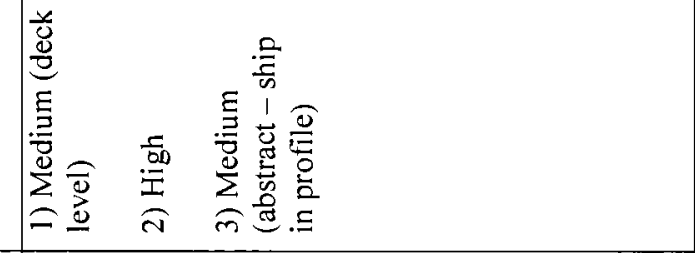 & 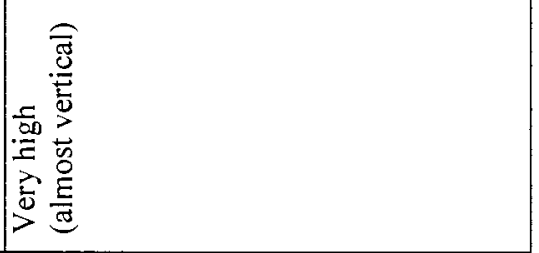 \\
\hline 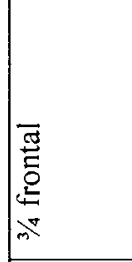 & 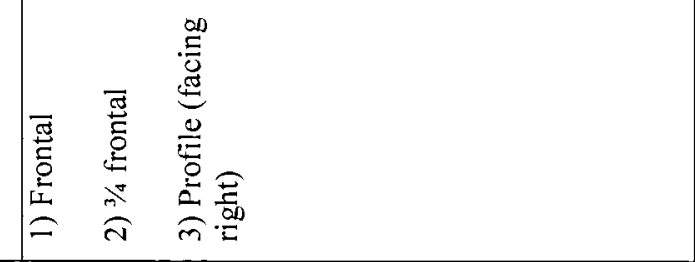 & 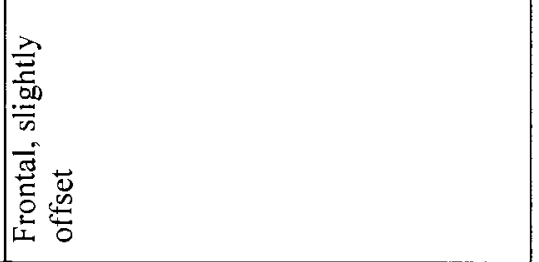 \\
\hline 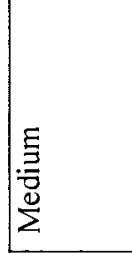 & 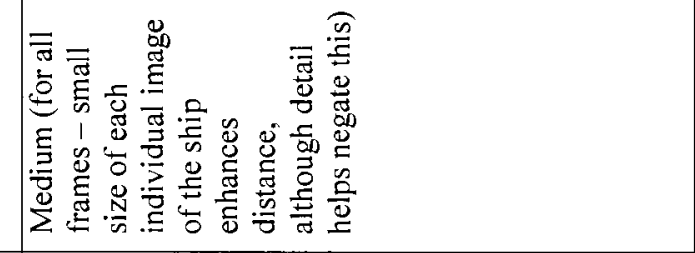 & 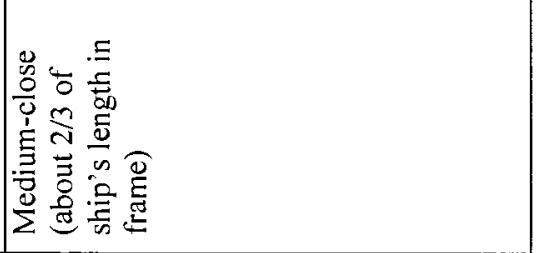 \\
\hline 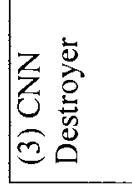 & 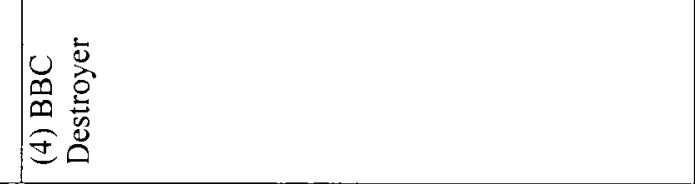 & 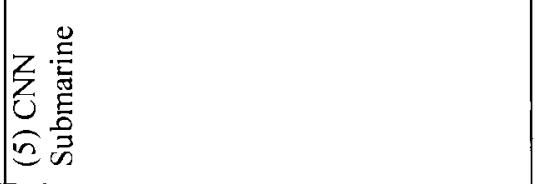 \\
\hline
\end{tabular}




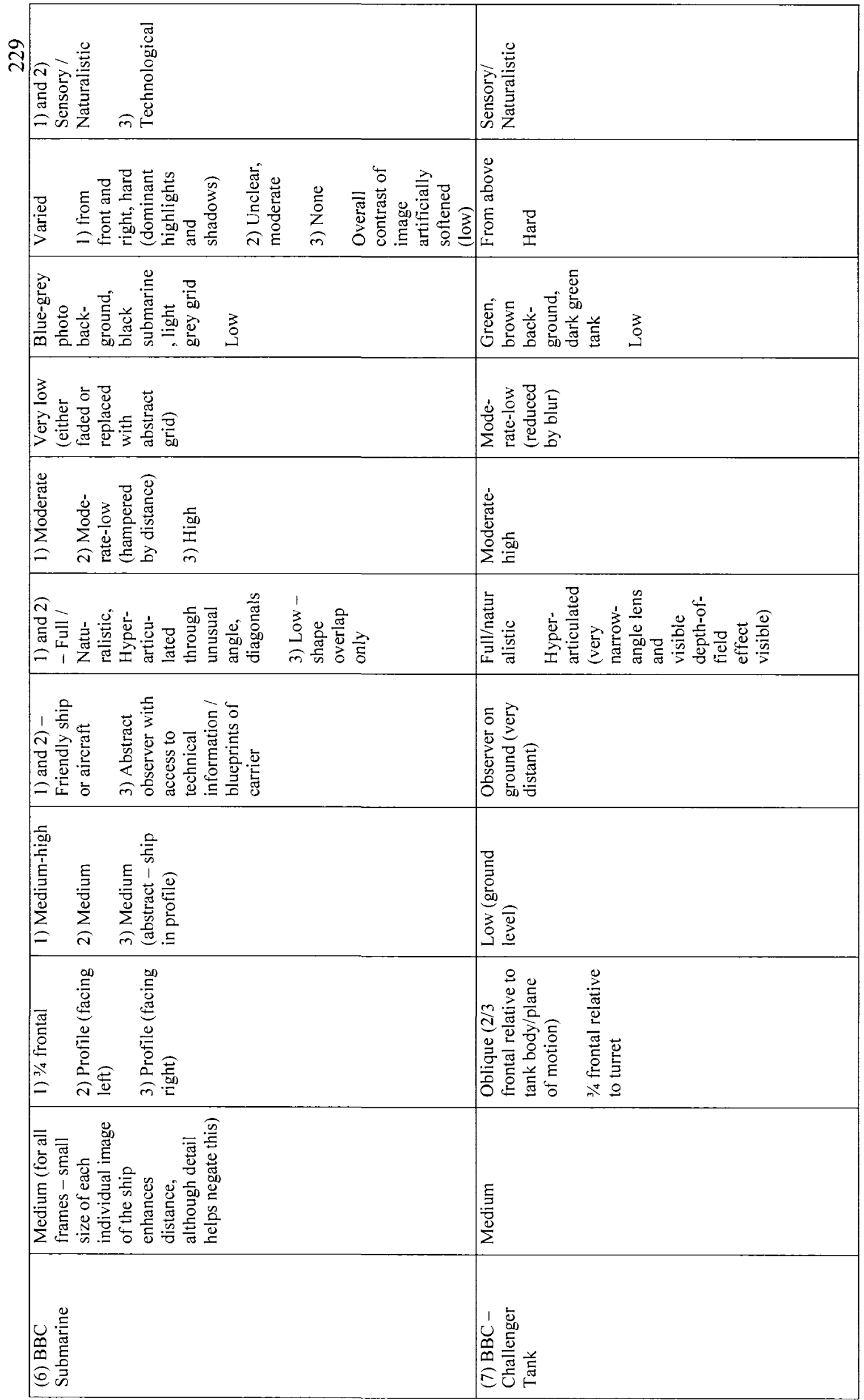




\begin{tabular}{|c|c|}
\hline 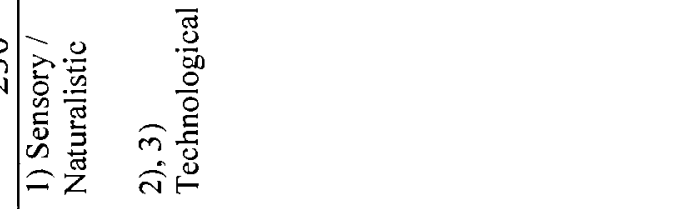 & 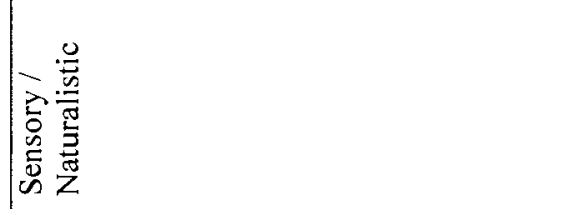 \\
\hline 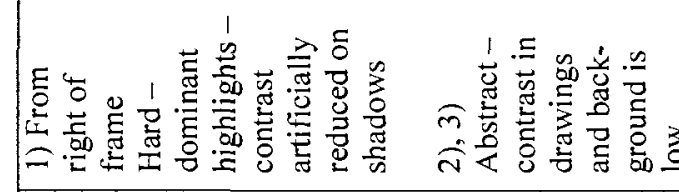 & 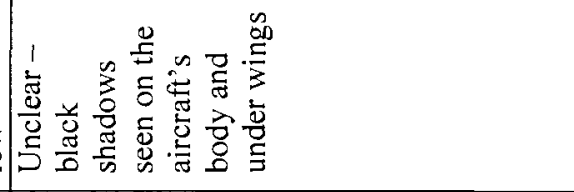 \\
\hline 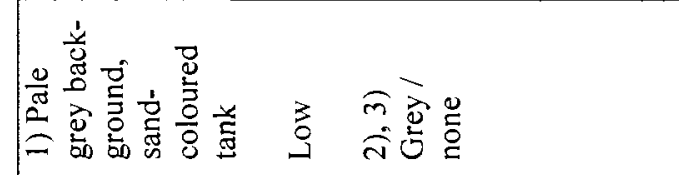 & 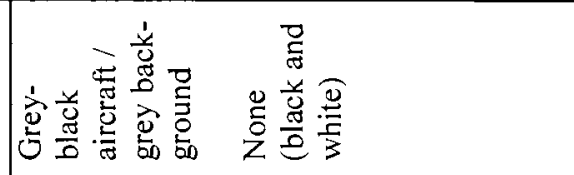 \\
\hline 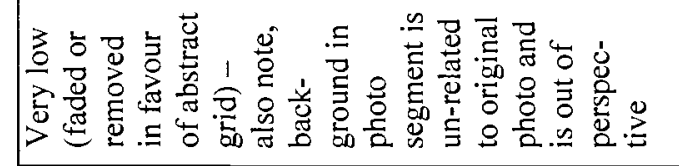 & 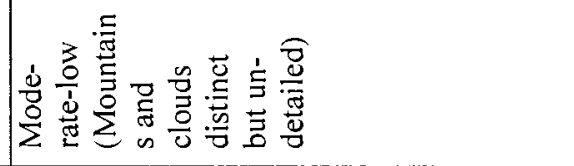 \\
\hline 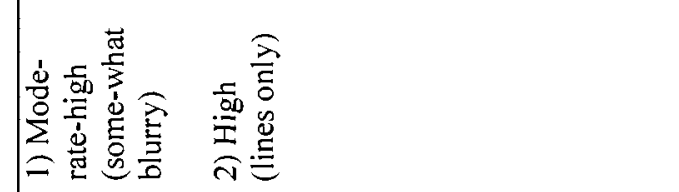 & 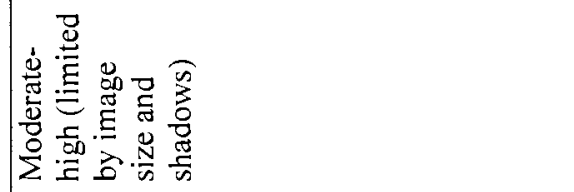 \\
\hline 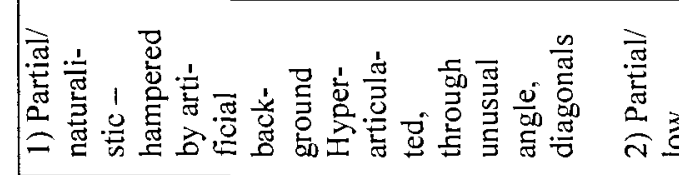 & 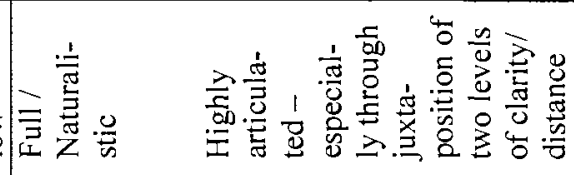 \\
\hline 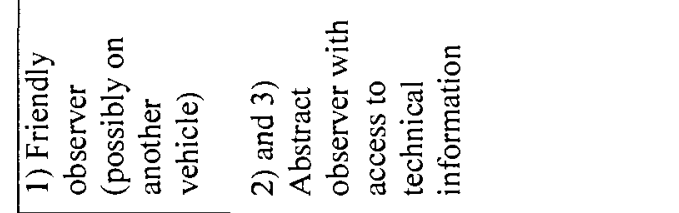 & 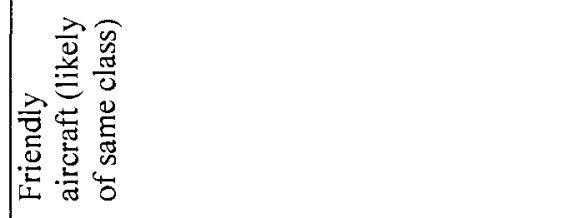 \\
\hline 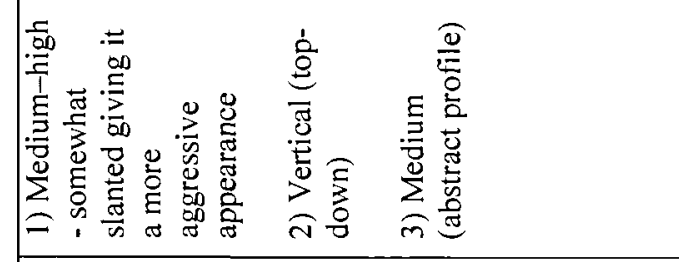 & 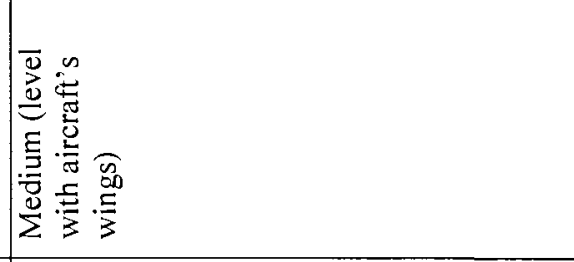 \\
\hline 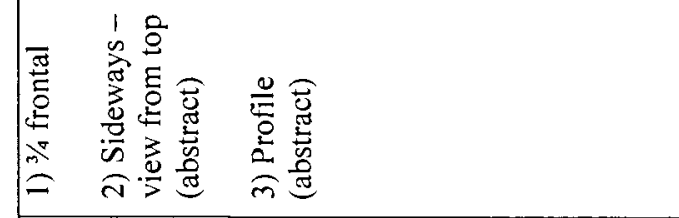 & 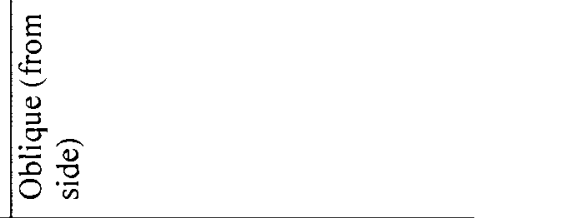 \\
\hline 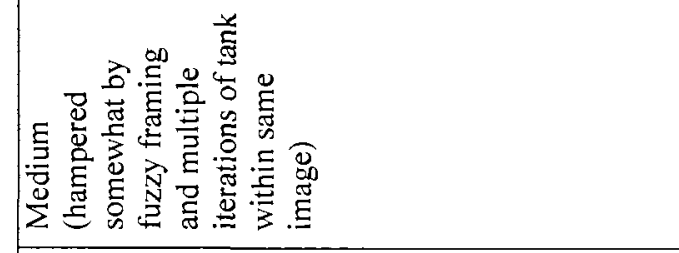 & 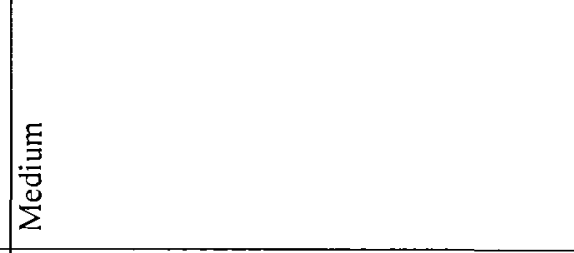 \\
\hline 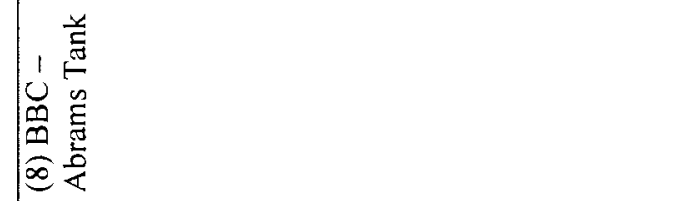 & 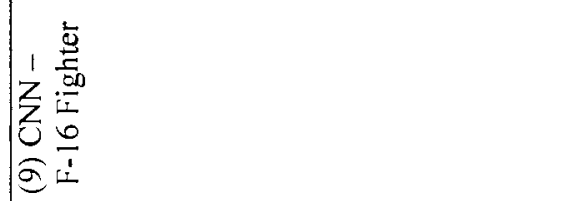 \\
\hline
\end{tabular}




\begin{tabular}{|c|c|c|}
\hline 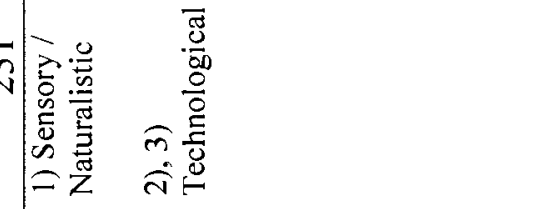 & 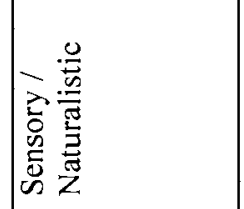 & 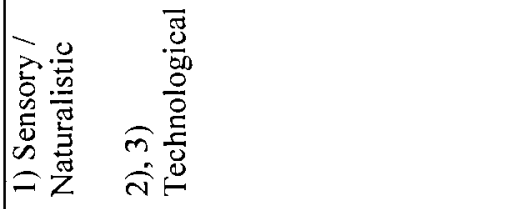 \\
\hline 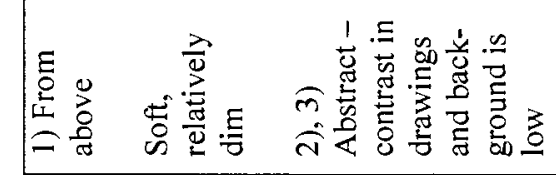 & 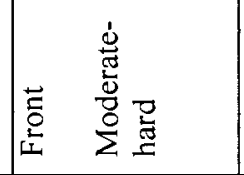 & 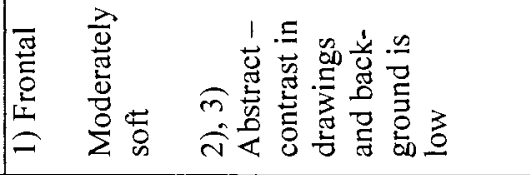 \\
\hline 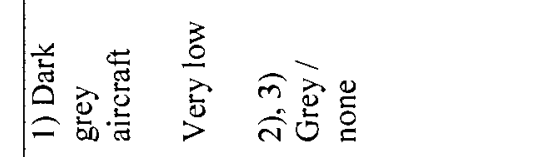 & 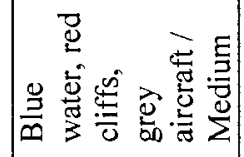 & 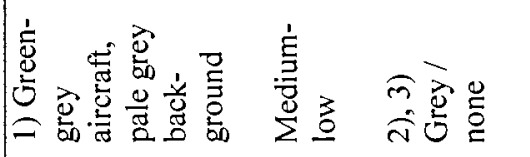 \\
\hline 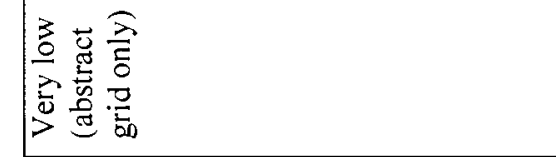 & 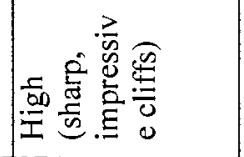 & 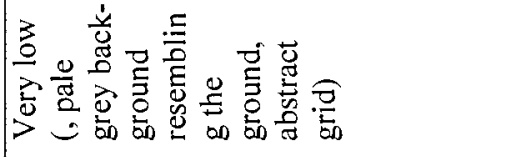 \\
\hline 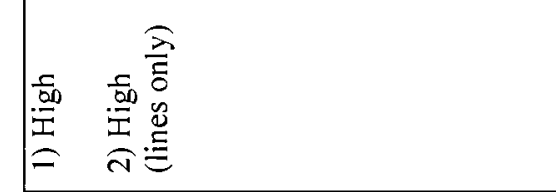 & $\frac{c}{\frac{c}{.00}}$ & 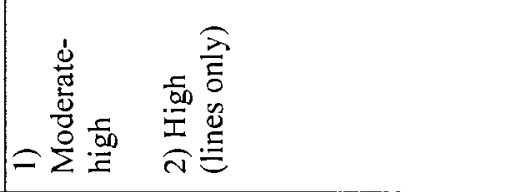 \\
\hline 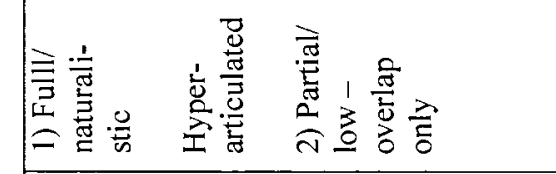 & 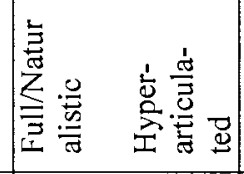 & 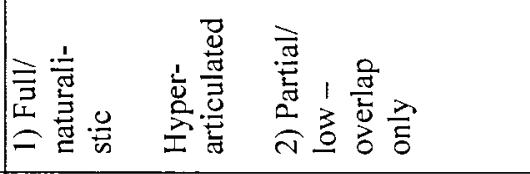 \\
\hline 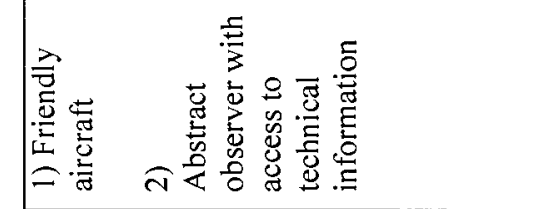 & 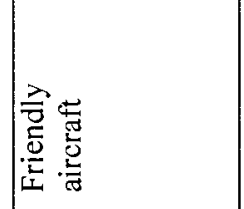 & 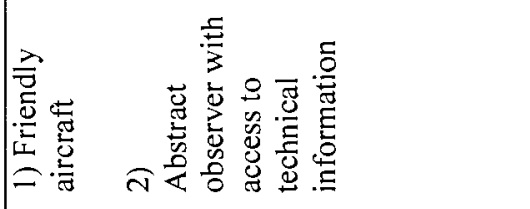 \\
\hline 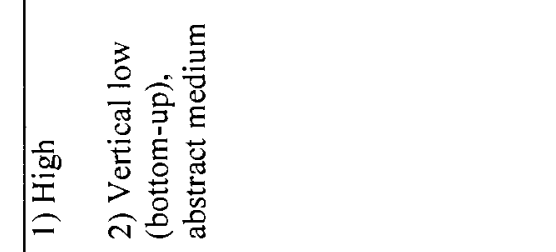 & 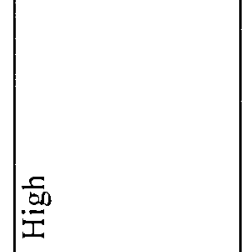 & 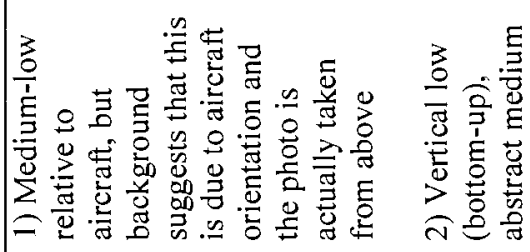 \\
\hline 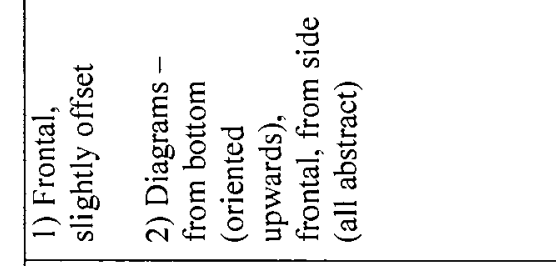 & 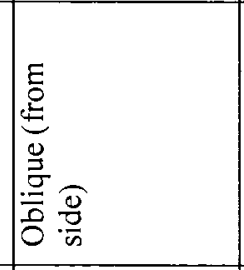 & 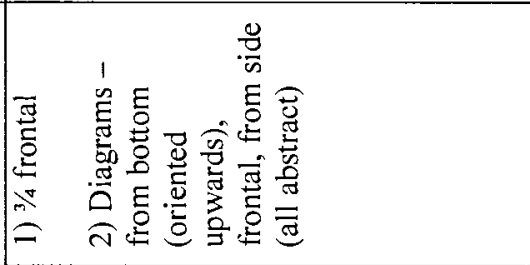 \\
\hline 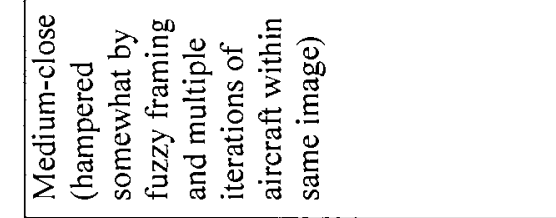 & E्ञ & 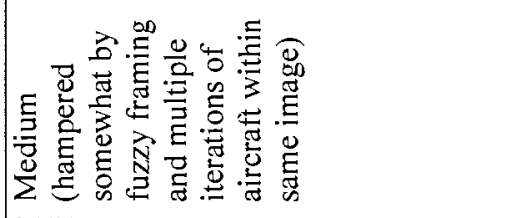 \\
\hline 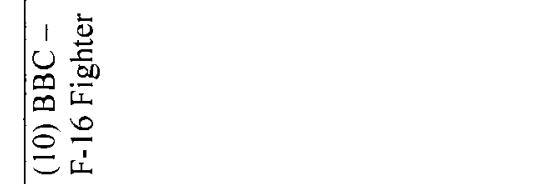 & 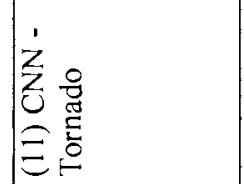 & 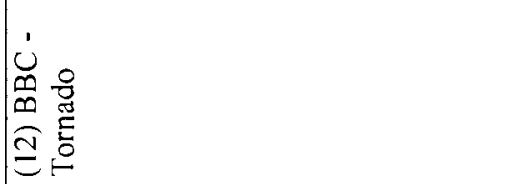 \\
\hline
\end{tabular}




\begin{tabular}{|c|c|c|}
\hline 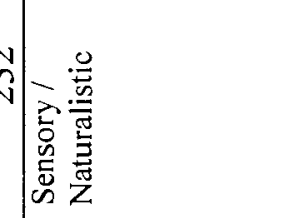 & 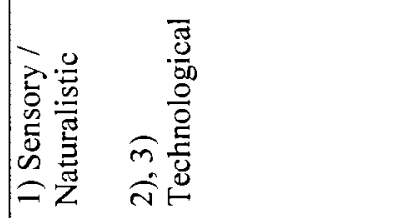 & \\
\hline 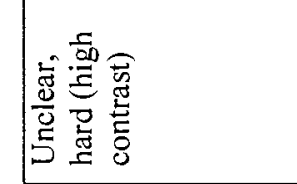 & 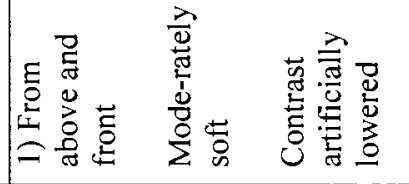 & 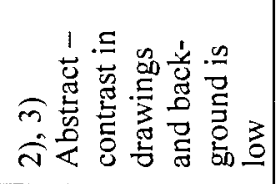 \\
\hline 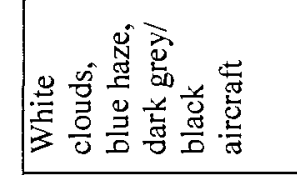 & 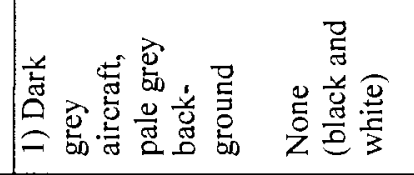 & 离离号 \\
\hline 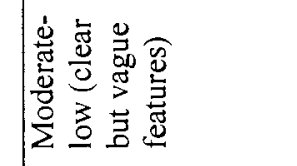 & 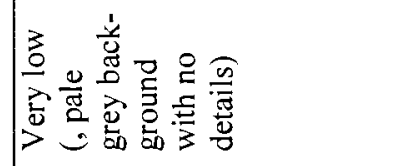 & \\
\hline 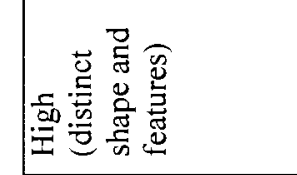 & 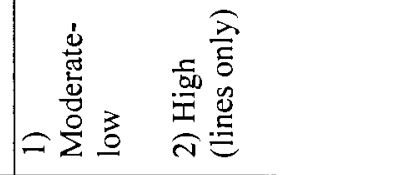 & \\
\hline 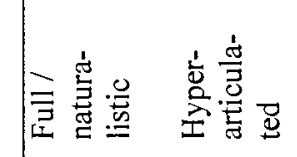 & 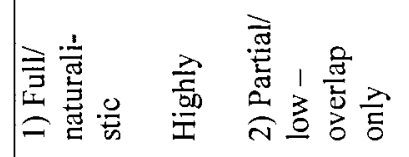 & \\
\hline 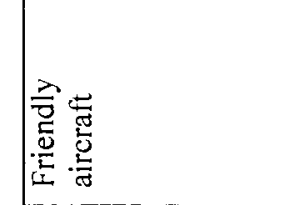 & 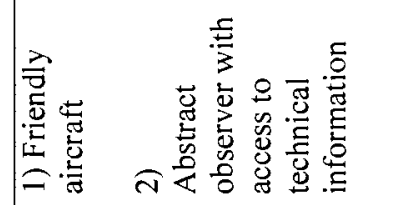 & \\
\hline 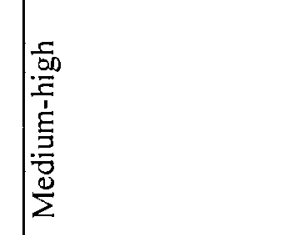 & 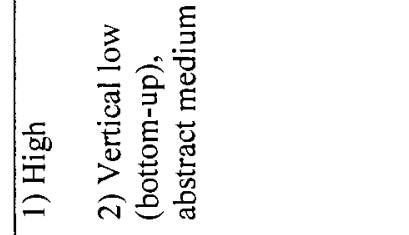 & \\
\hline 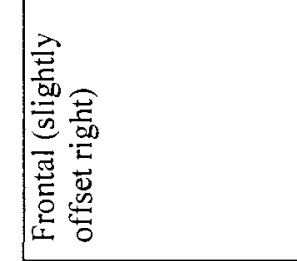 & 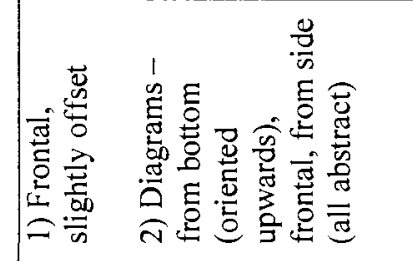 & \\
\hline 立 & 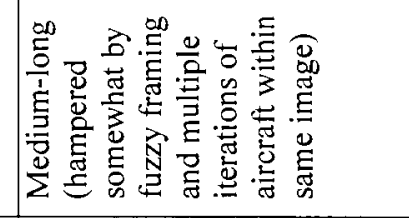 & \\
\hline 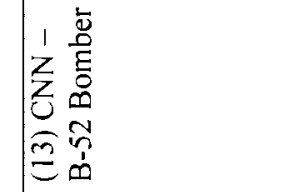 & 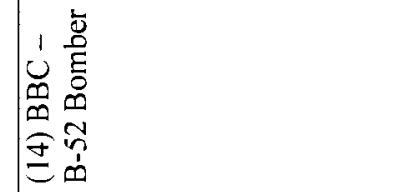 & \\
\hline
\end{tabular}


$\stackrel{m}{\pi}$

\begin{tabular}{|c|c|c|}
\hline \multicolumn{3}{|c|}{ 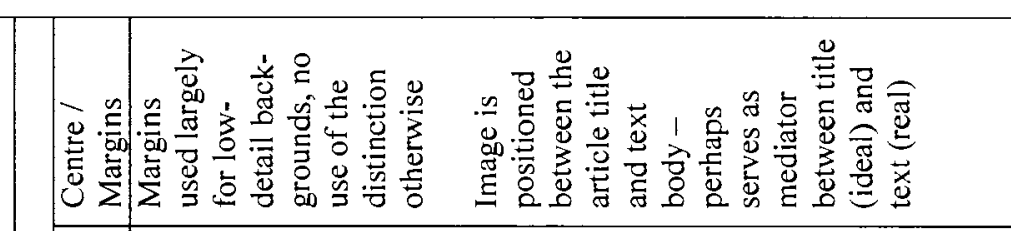 } \\
\hline 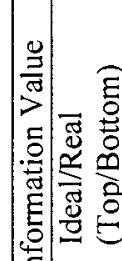 & 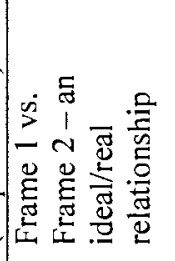 & 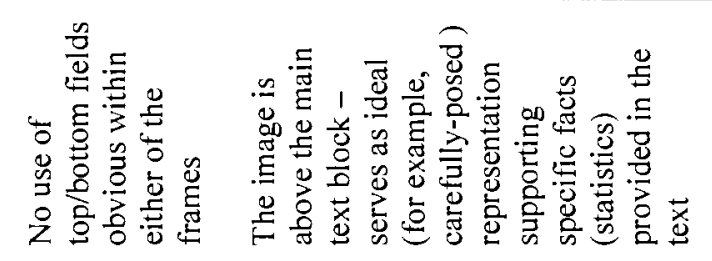 \\
\hline
\end{tabular}

든

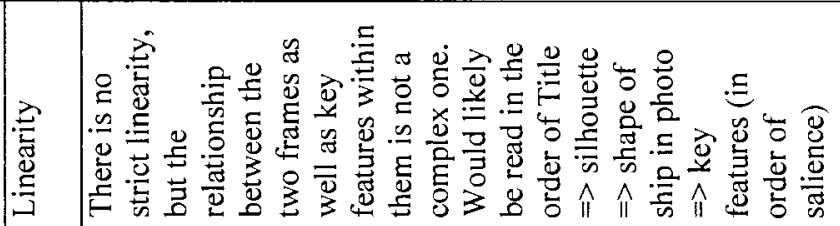

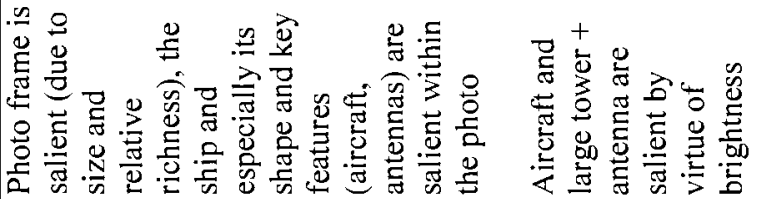

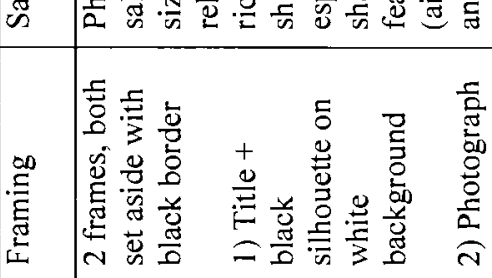

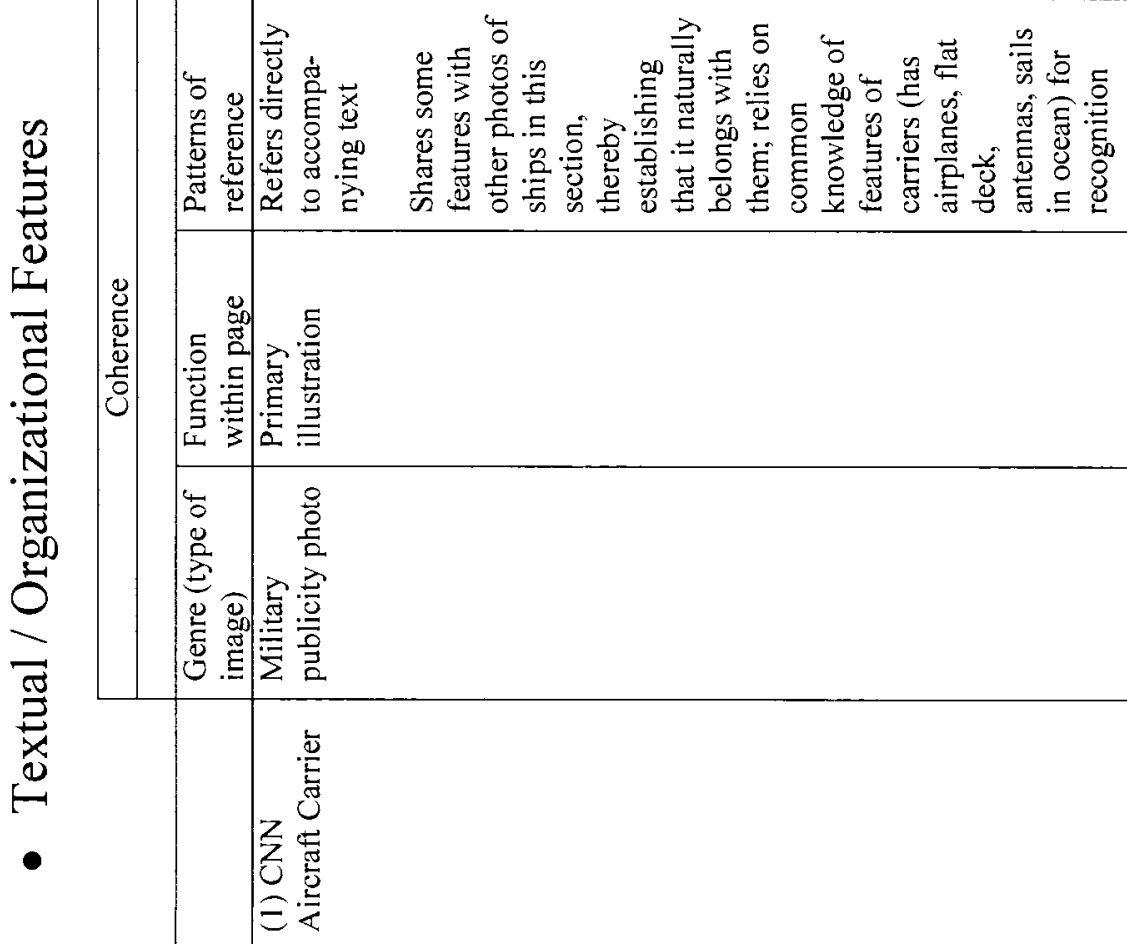




\begin{tabular}{|c|c|c|}
\hline 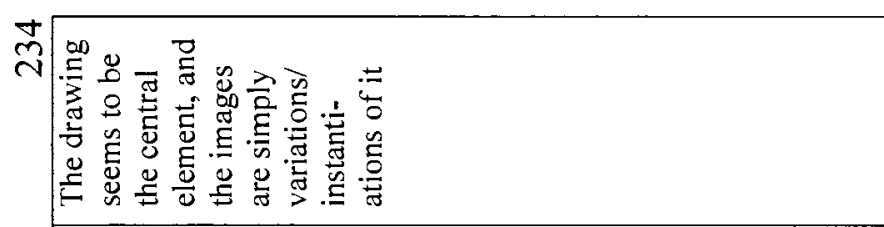 & 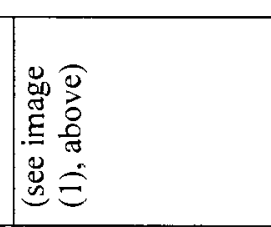 & 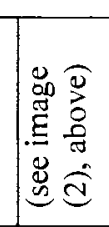 \\
\hline 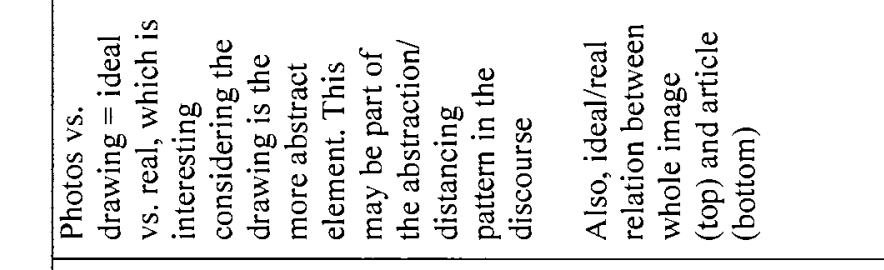 & 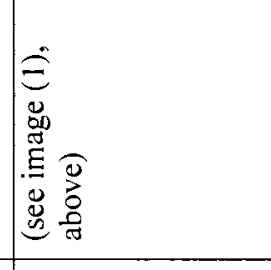 & 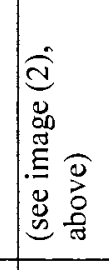 \\
\hline 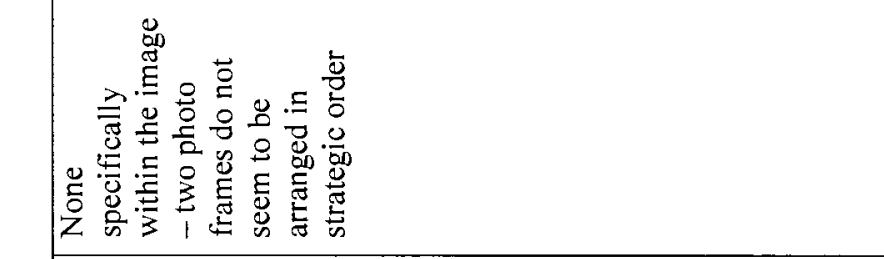 & 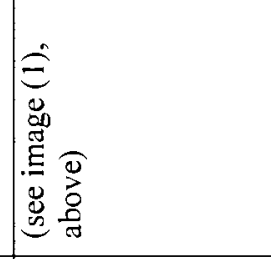 & 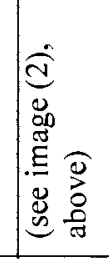 \\
\hline 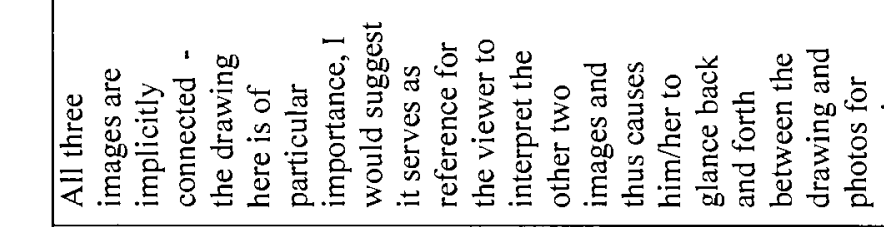 & 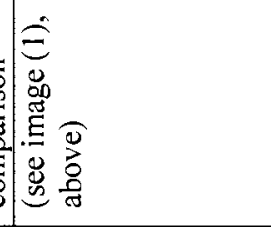 & 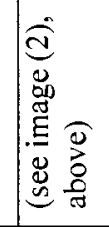 \\
\hline 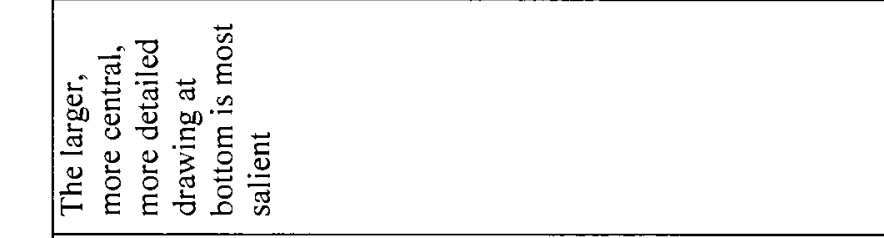 & 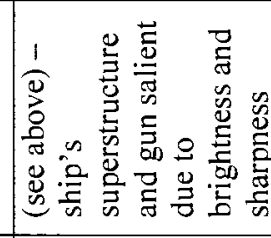 & 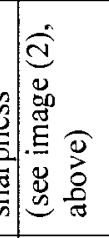 \\
\hline 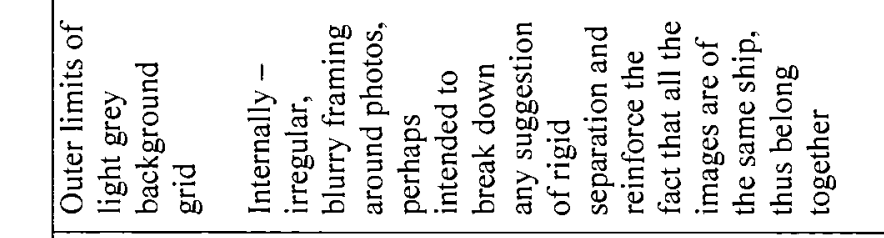 & 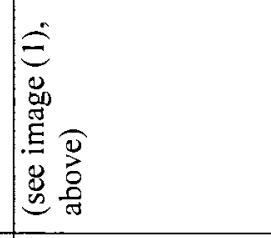 & 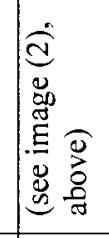 \\
\hline 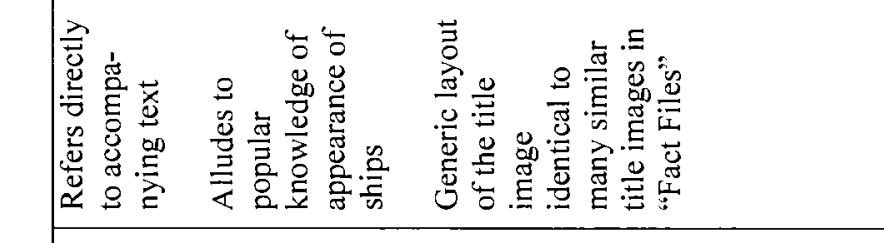 & 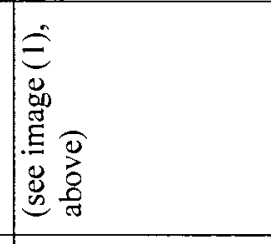 & 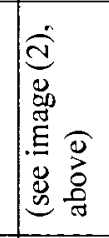 \\
\hline 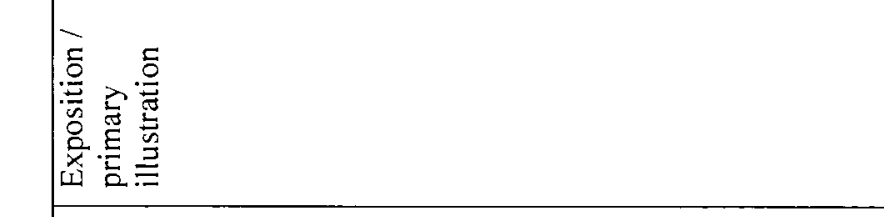 & 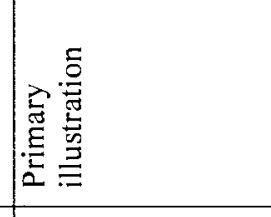 & 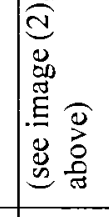 \\
\hline 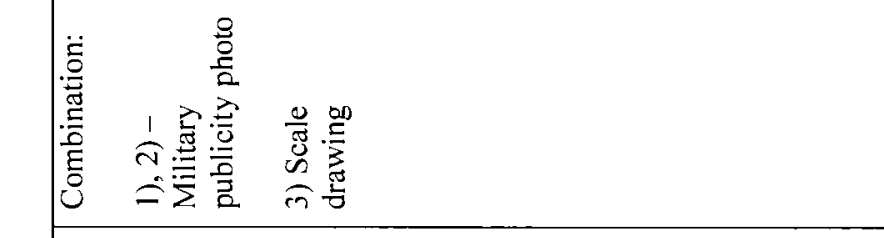 & 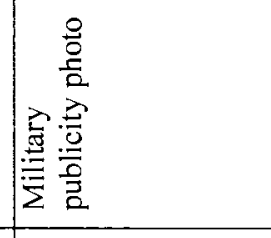 & 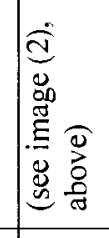 \\
\hline 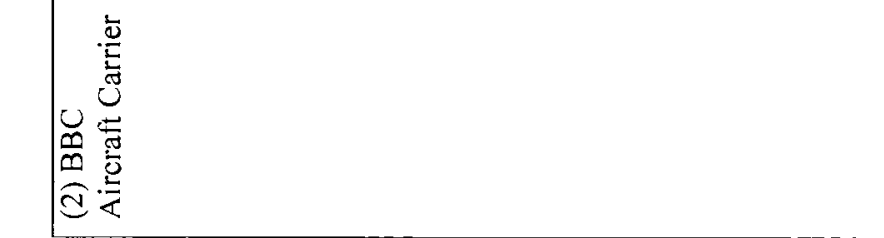 & 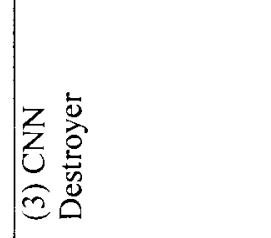 & 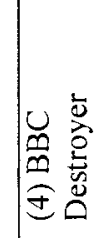 \\
\hline
\end{tabular}




\begin{tabular}{|c|c|}
\hline 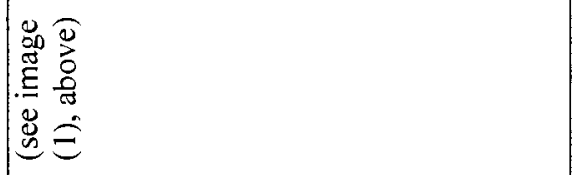 & 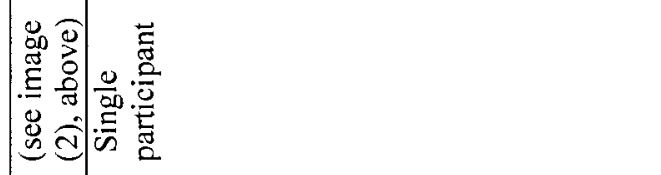 \\
\hline 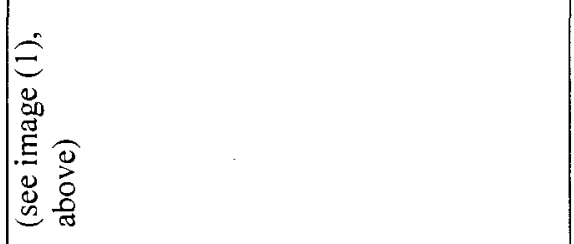 & 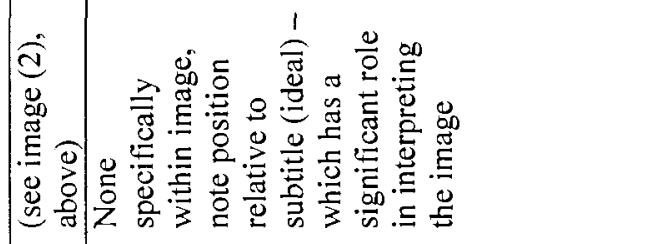 \\
\hline 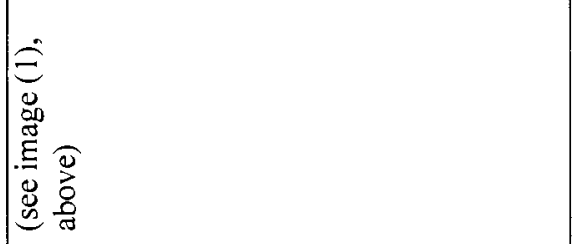 & 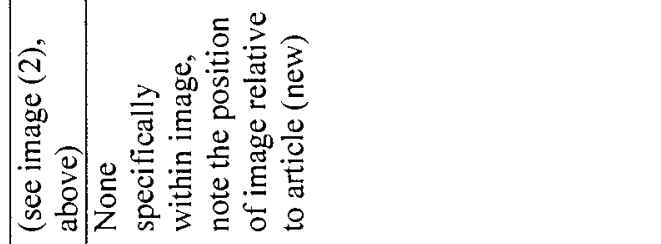 \\
\hline 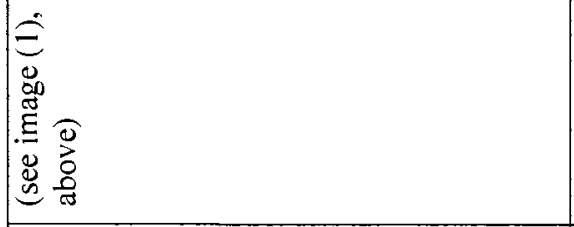 & 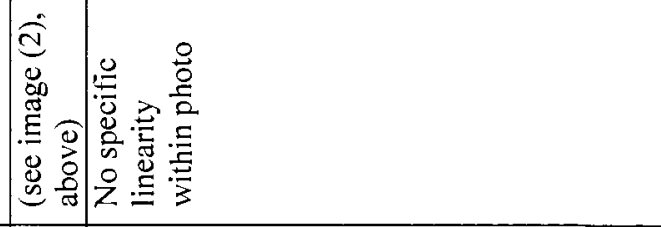 \\
\hline 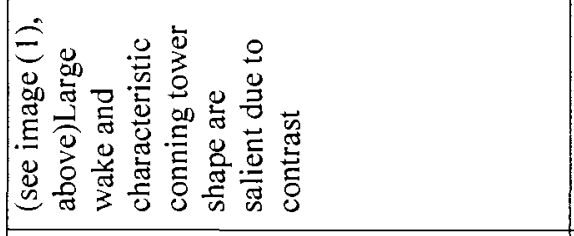 & 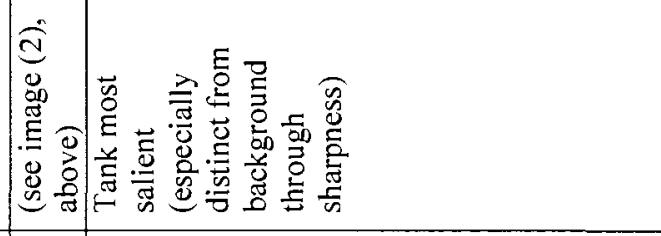 \\
\hline 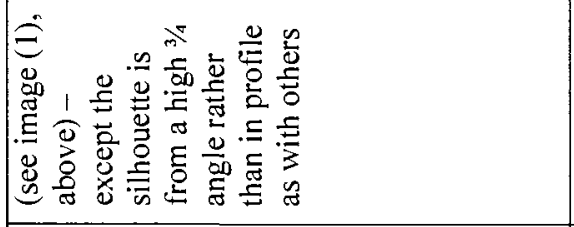 & 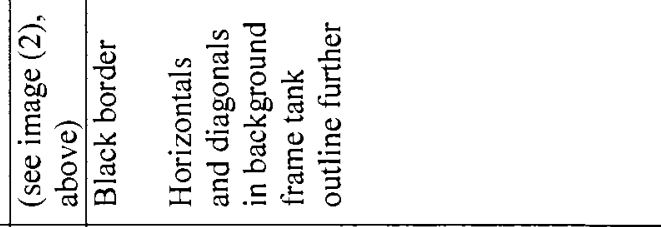 \\
\hline 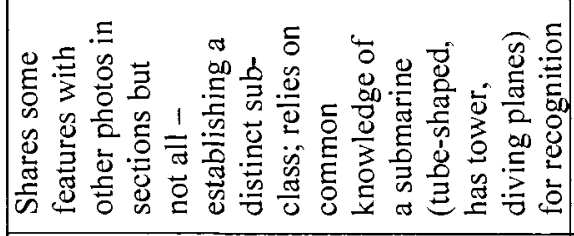 & 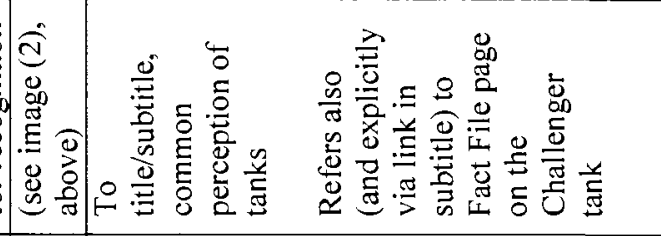 \\
\hline 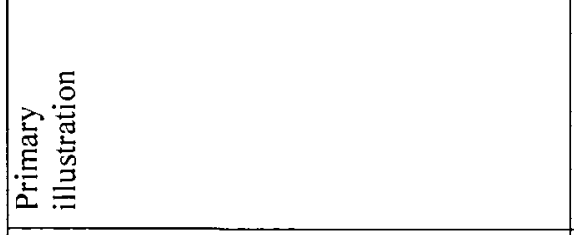 & 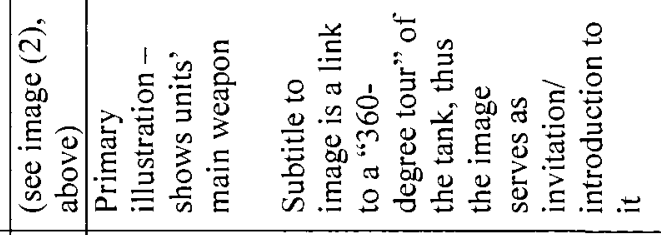 \\
\hline 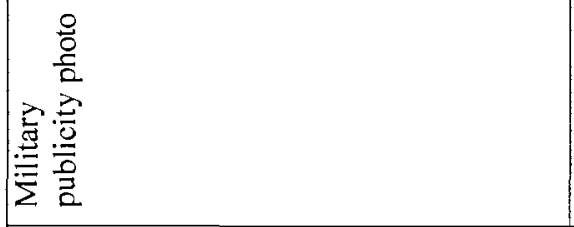 & 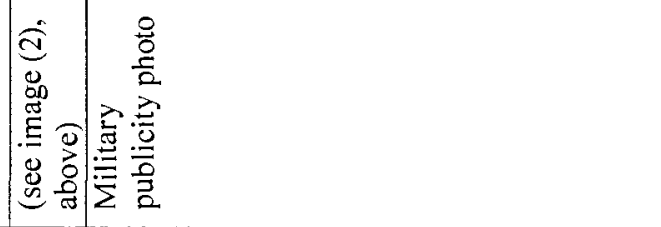 \\
\hline 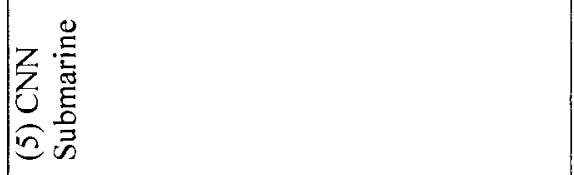 & 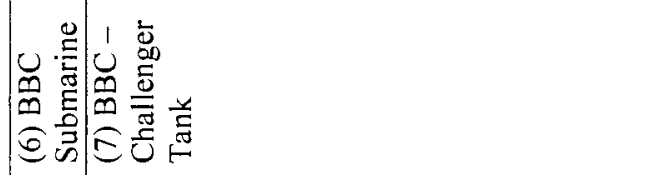 \\
\hline
\end{tabular}




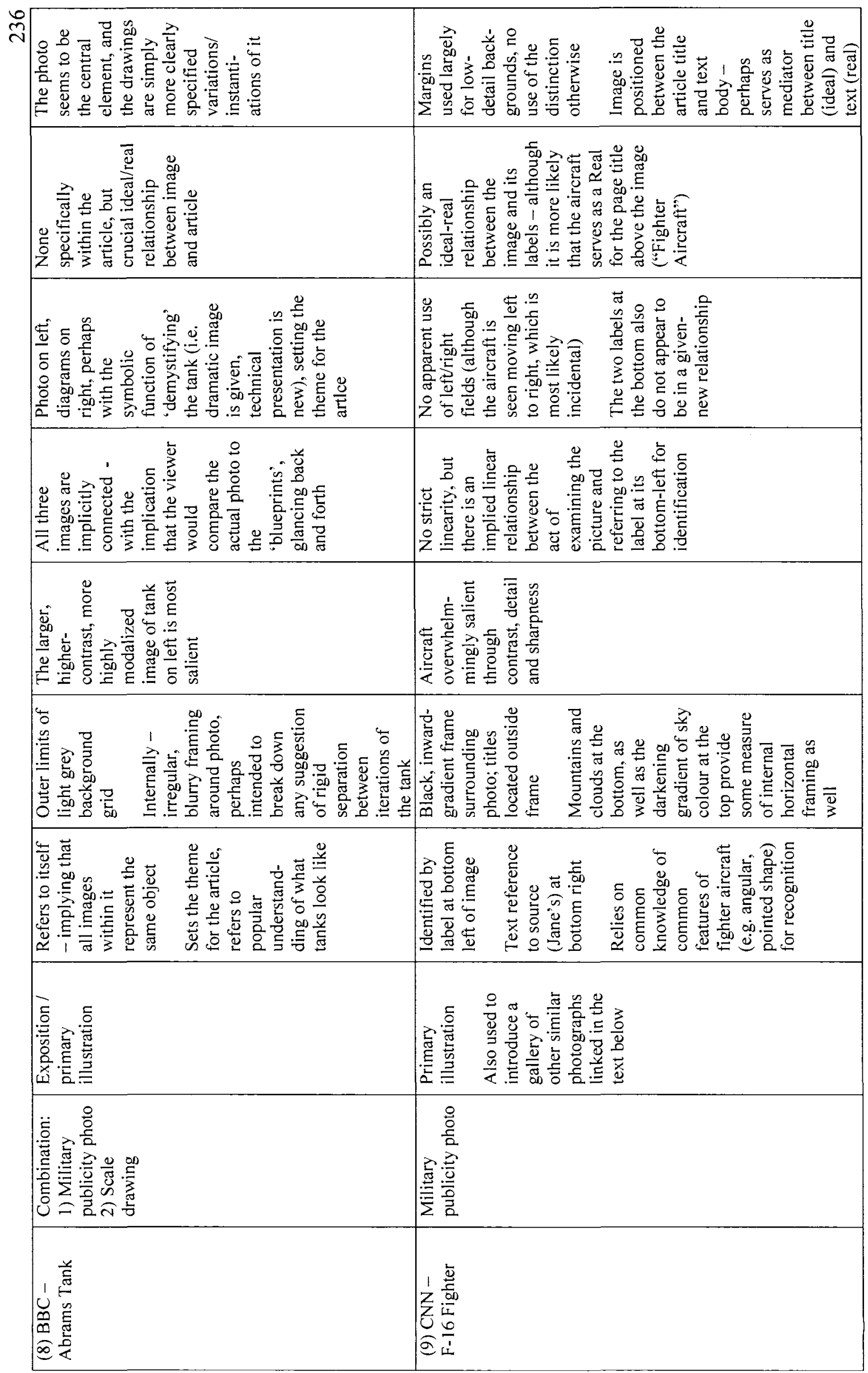


ลิ

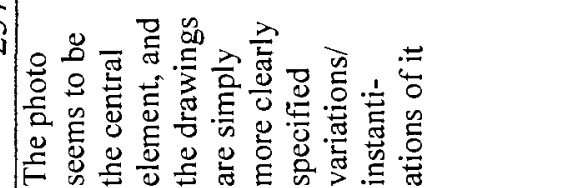

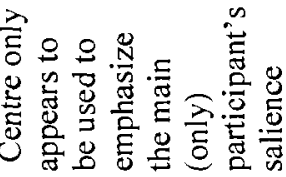

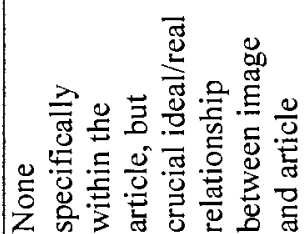

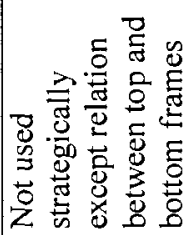

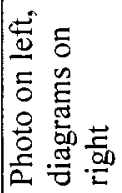

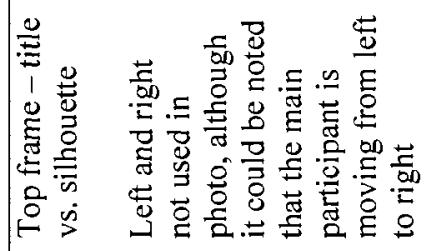

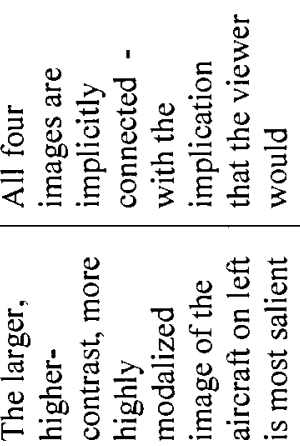

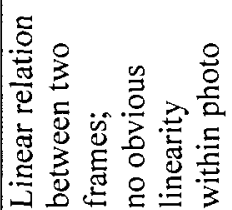

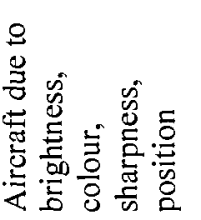

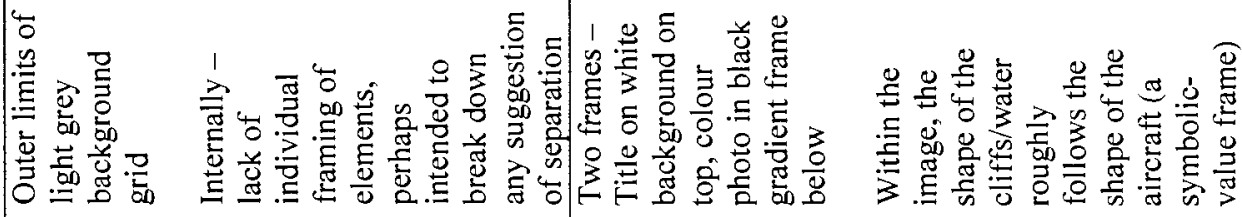

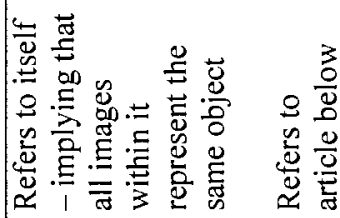

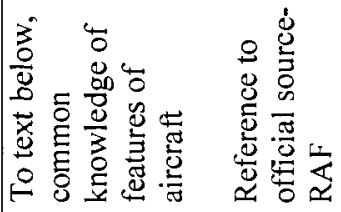

\section{站

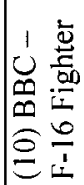

\begin{tabular}{|c|}
\hline 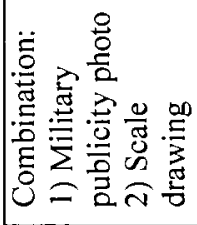 \\
\hline
\end{tabular}




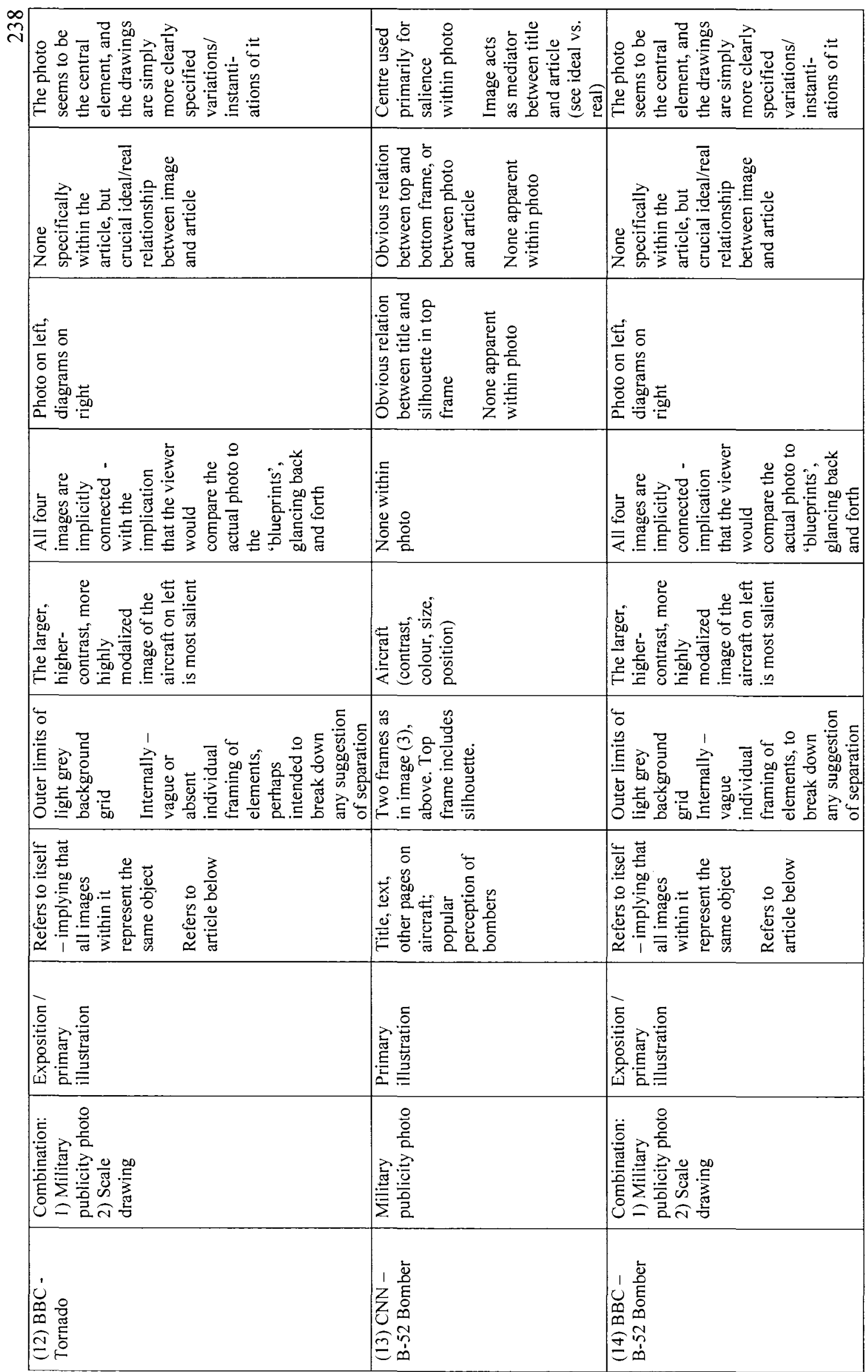




\begin{tabular}{|c|c|c|c|c|}
\hline & & | & 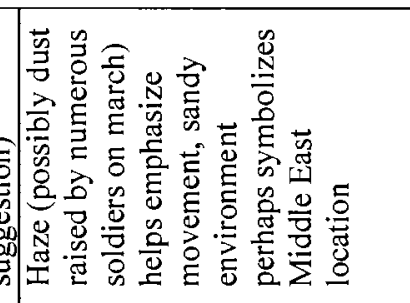 & 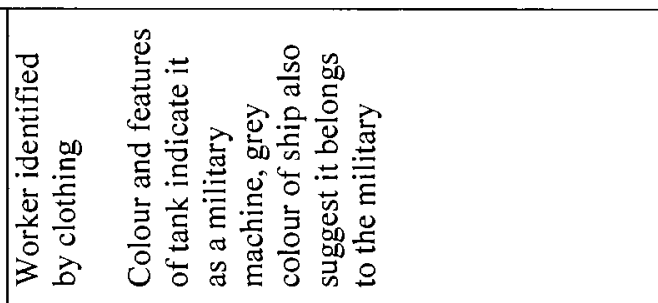 \\
\hline & & 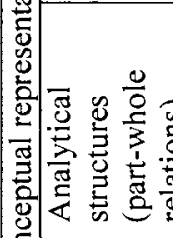 & 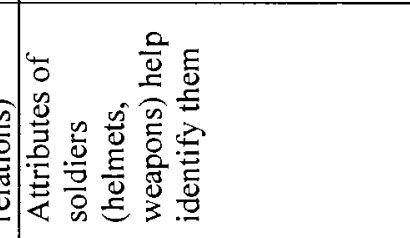 & 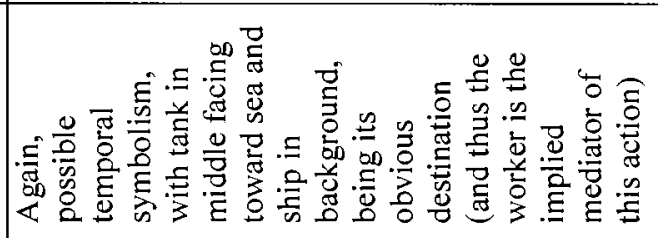 \\
\hline & & 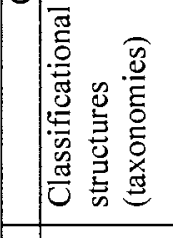 & 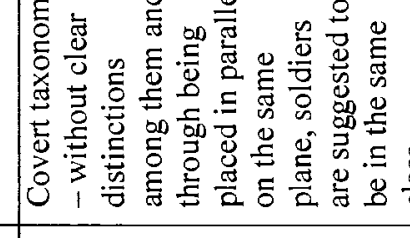 & 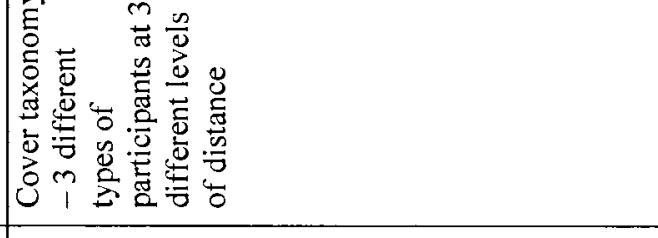 \\
\hline & & 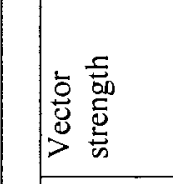 & 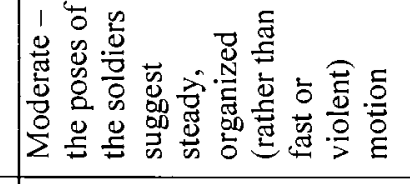 & 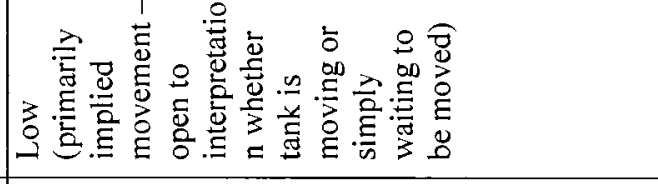 \\
\hline & & 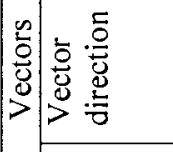 & 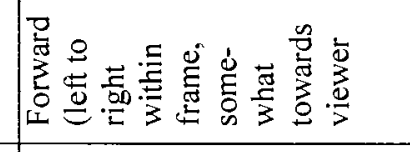 & 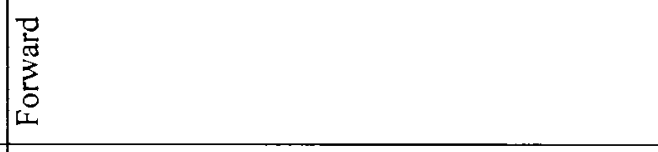 \\
\hline & & 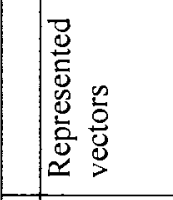 & 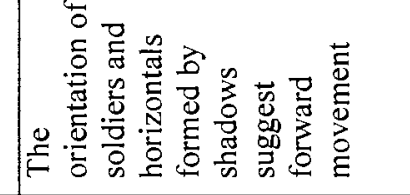 & 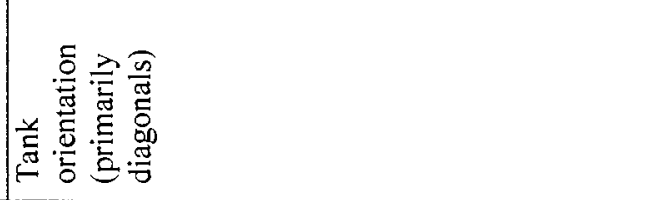 \\
\hline & $\stackrel{\mathscr{D}}{\stackrel{\mathscr{Z}}{\Xi}}$ & 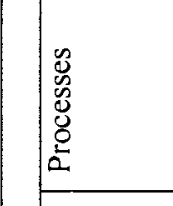 & 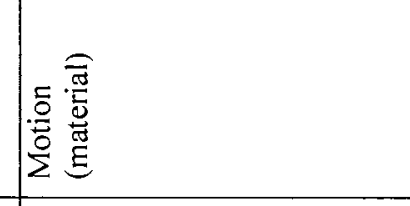 & 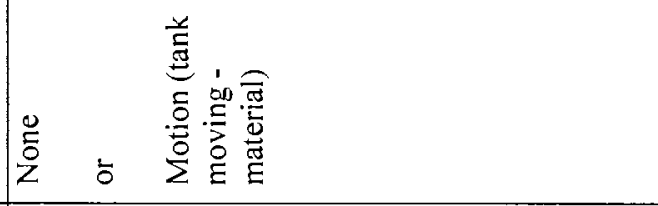 \\
\hline 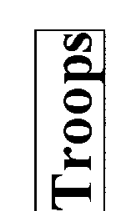 & 宸 & 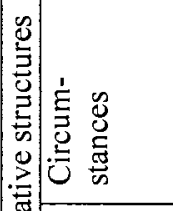 & 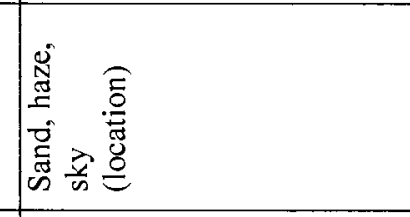 & 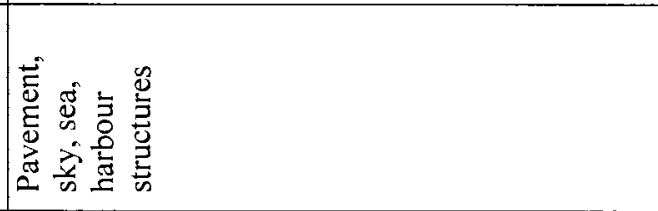 \\
\hline 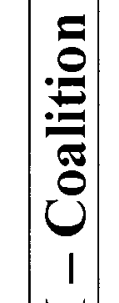 & 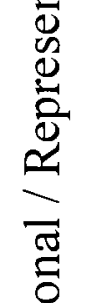 & 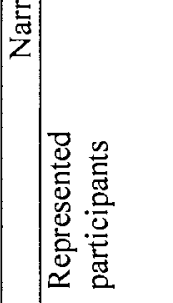 & 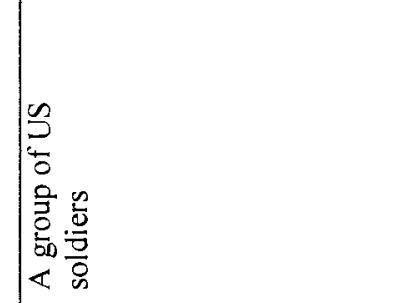 & 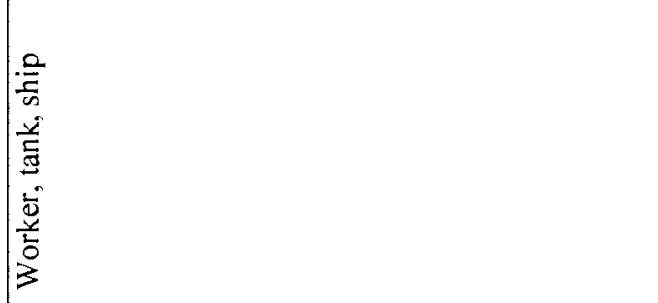 \\
\hline 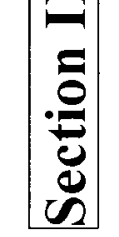 & 营 & & 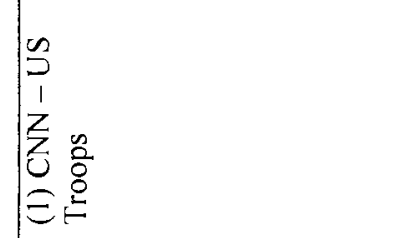 & 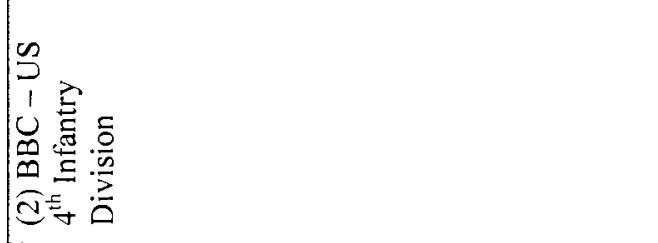 \\
\hline
\end{tabular}




\begin{tabular}{|c|c|c|}
\hline 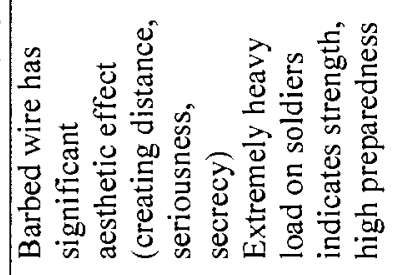 & 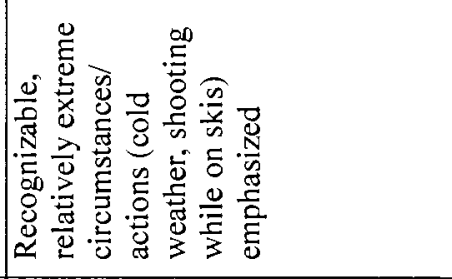 & 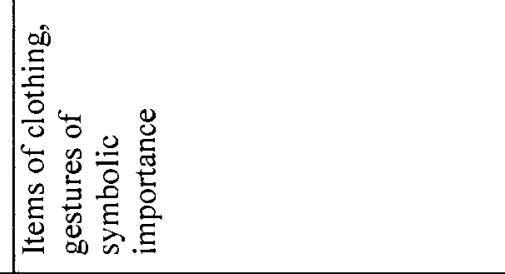 \\
\hline 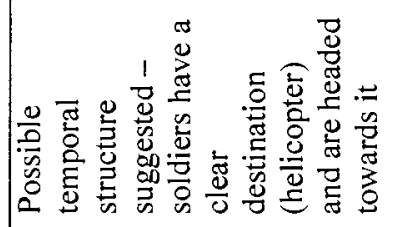 & 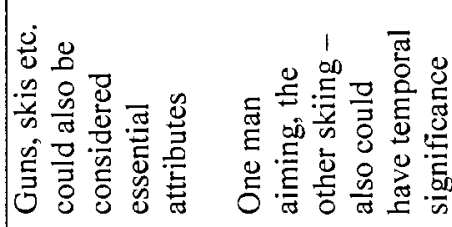 & 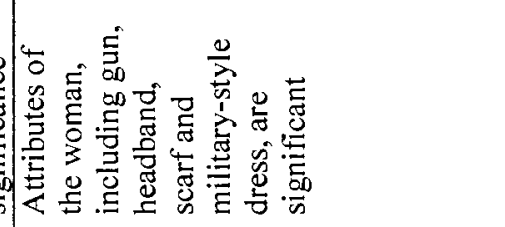 \\
\hline 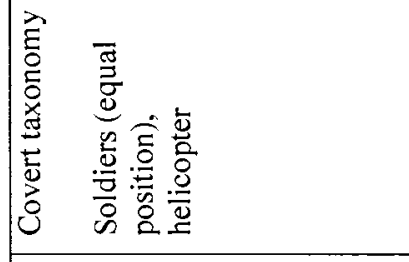 & 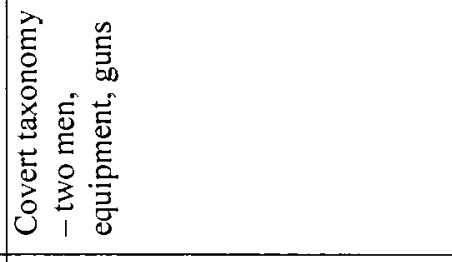 & 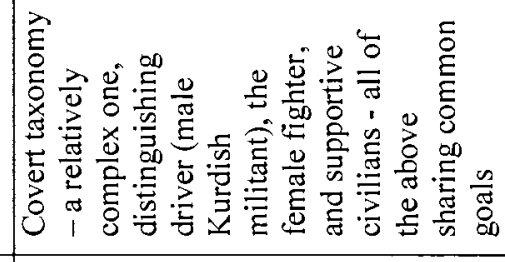 \\
\hline 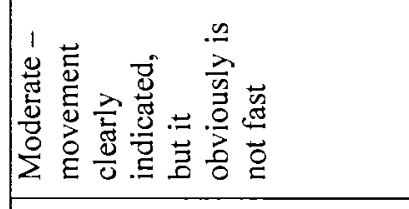 & 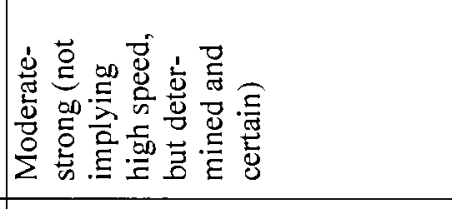 & 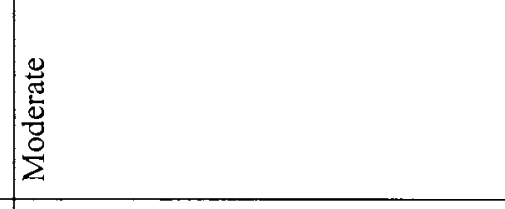 \\
\hline 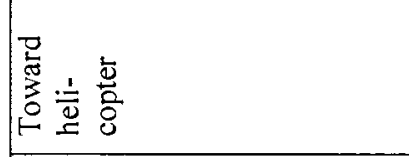 & 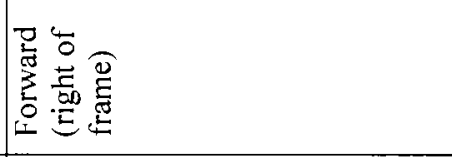 & 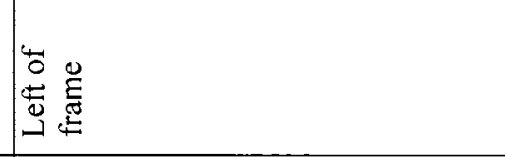 \\
\hline 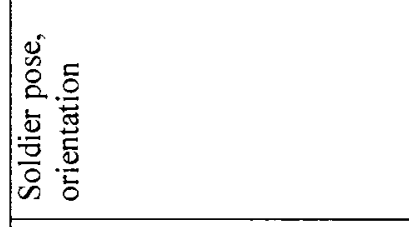 & 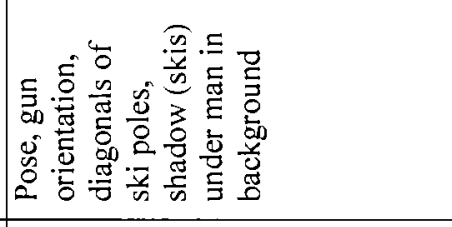 & 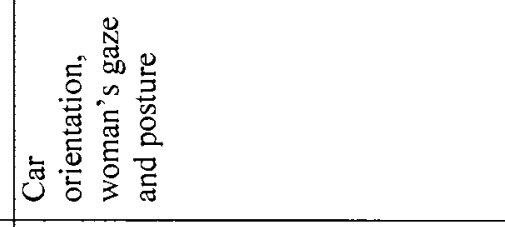 \\
\hline 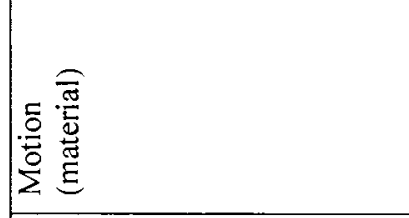 & 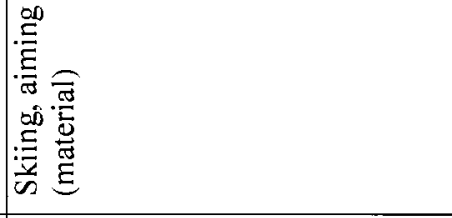 & 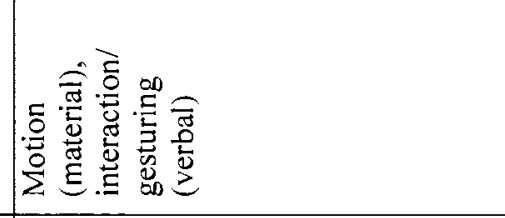 \\
\hline 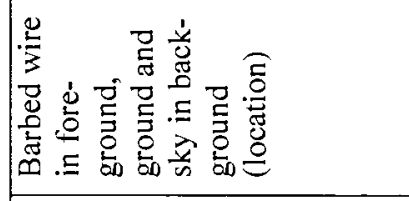 & 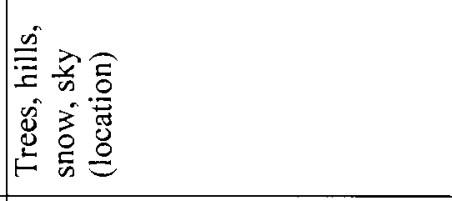 & 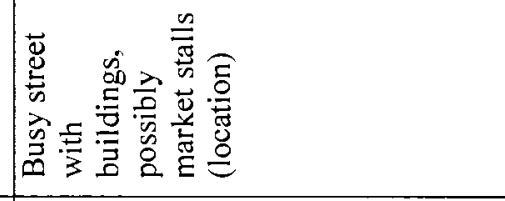 \\
\hline 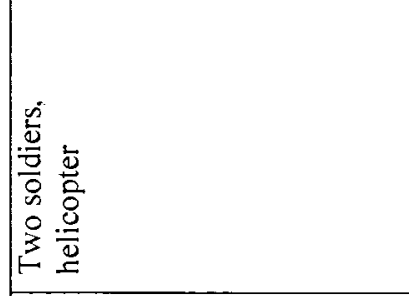 & 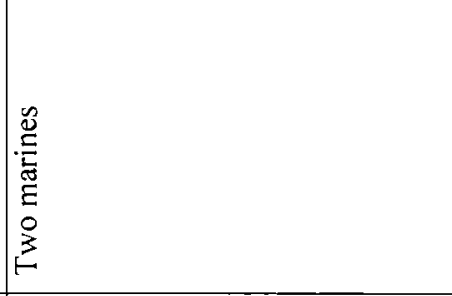 & 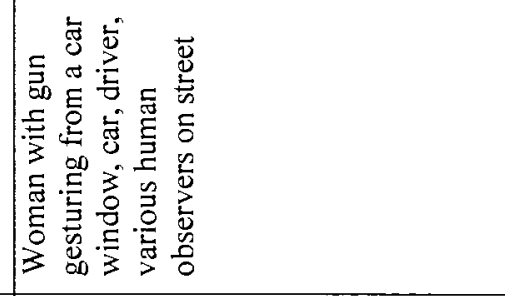 \\
\hline 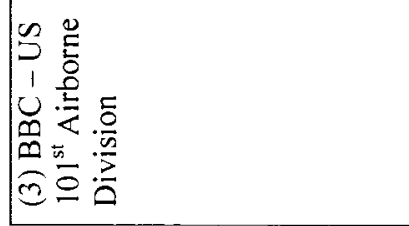 & 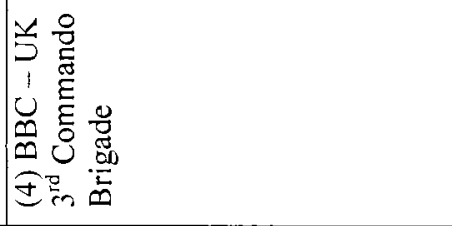 & 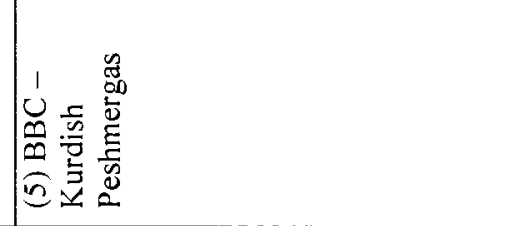 \\
\hline
\end{tabular}




\begin{tabular}{|c|c|c|}
\hline 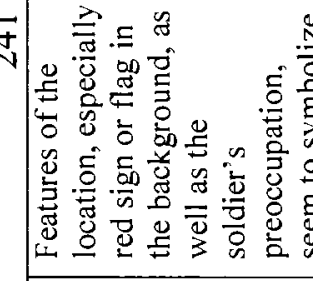 & 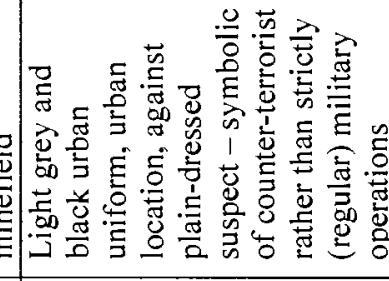 & 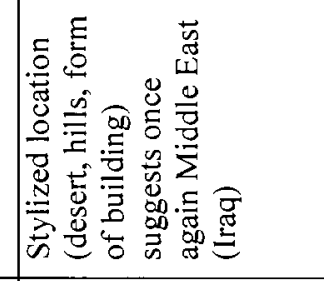 \\
\hline 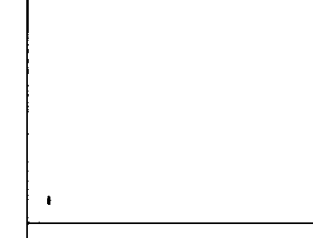 & 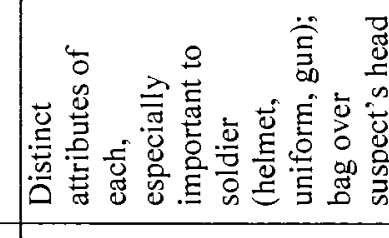 & 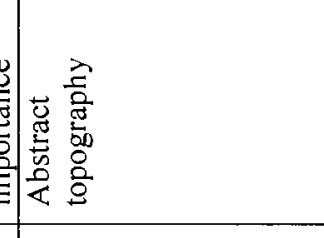 \\
\hline i & 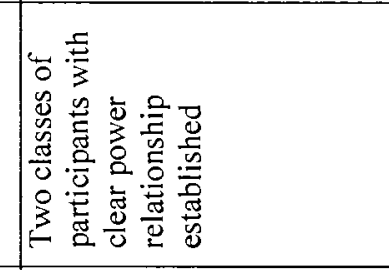 & 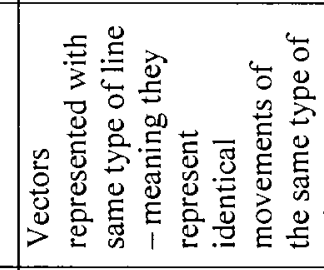 \\
\hline 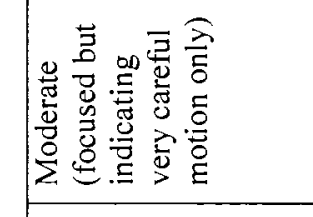 & 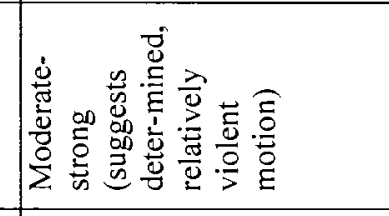 & 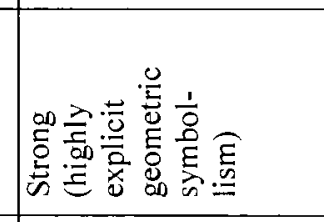 \\
\hline 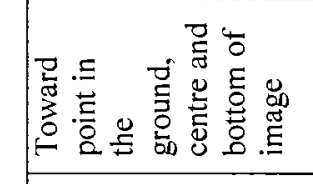 & 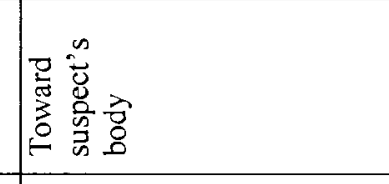 & 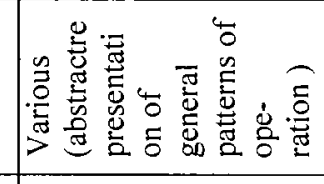 \\
\hline 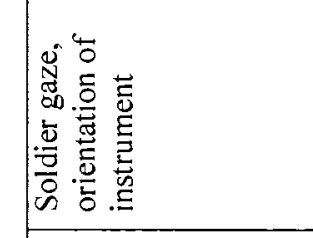 & 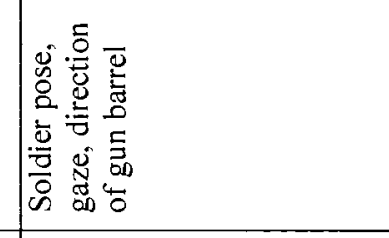 & 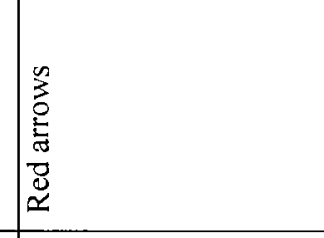 \\
\hline 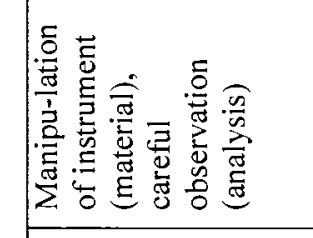 & 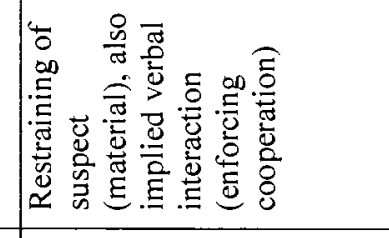 & 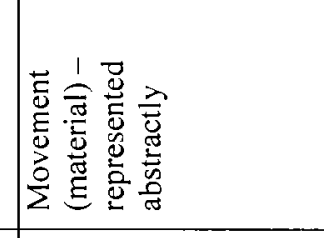 \\
\hline 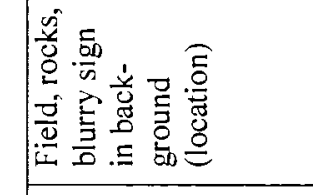 & 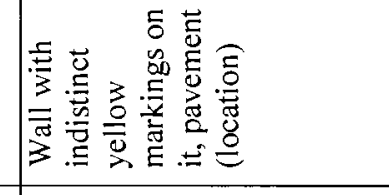 & 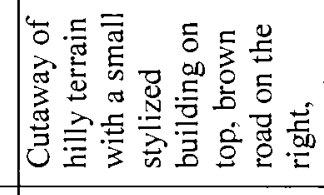 \\
\hline 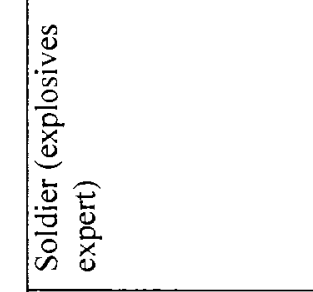 & 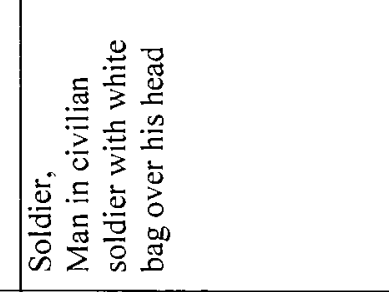 & 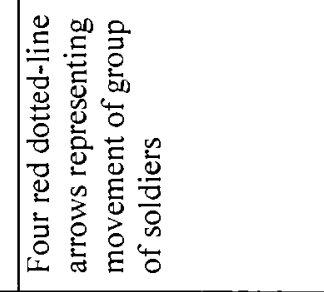 \\
\hline 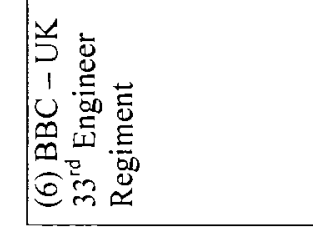 & 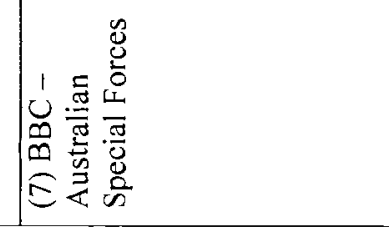 & 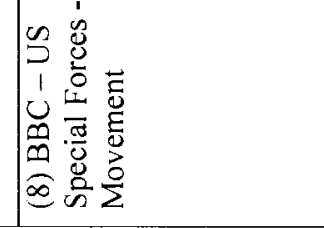 \\
\hline
\end{tabular}




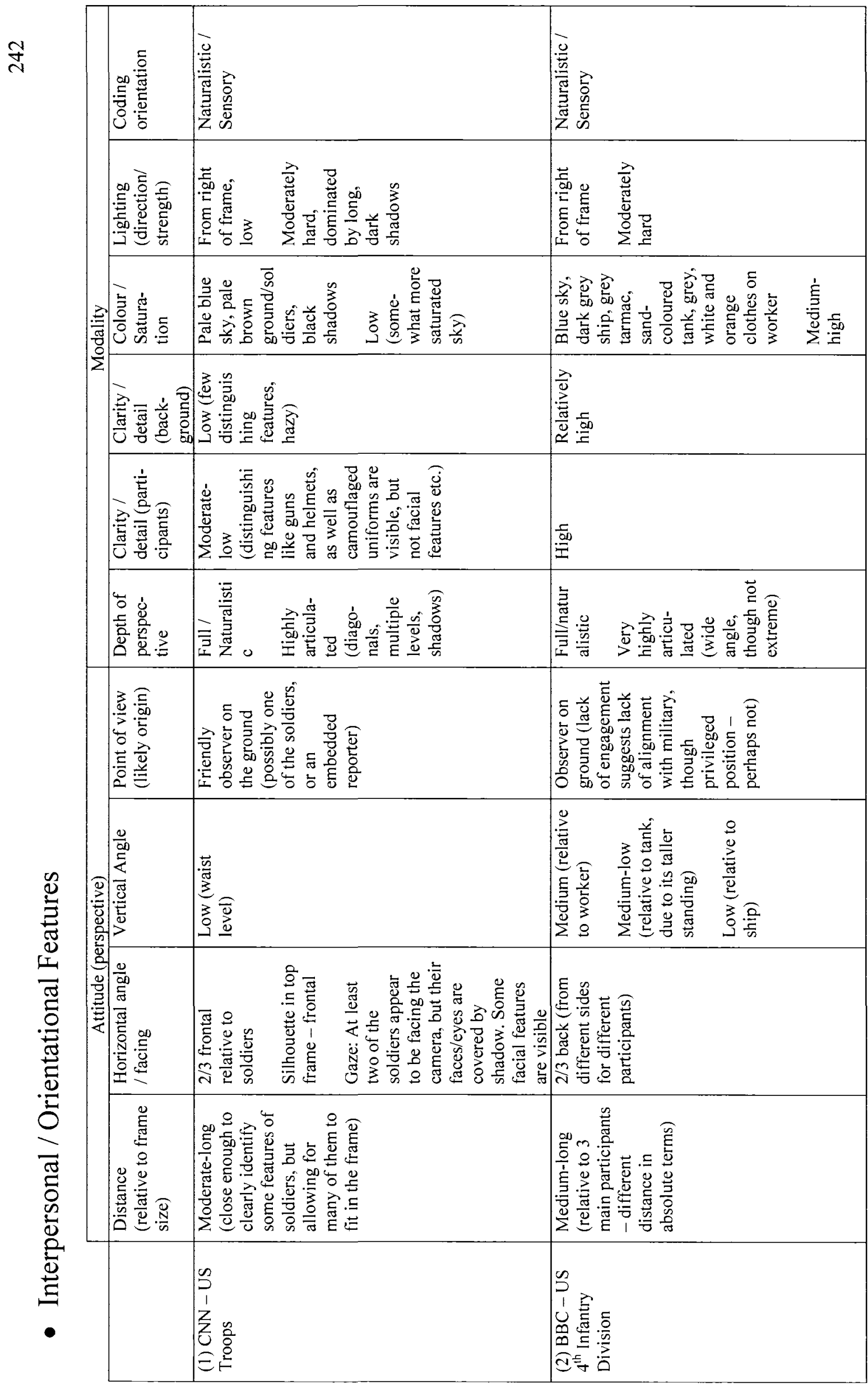




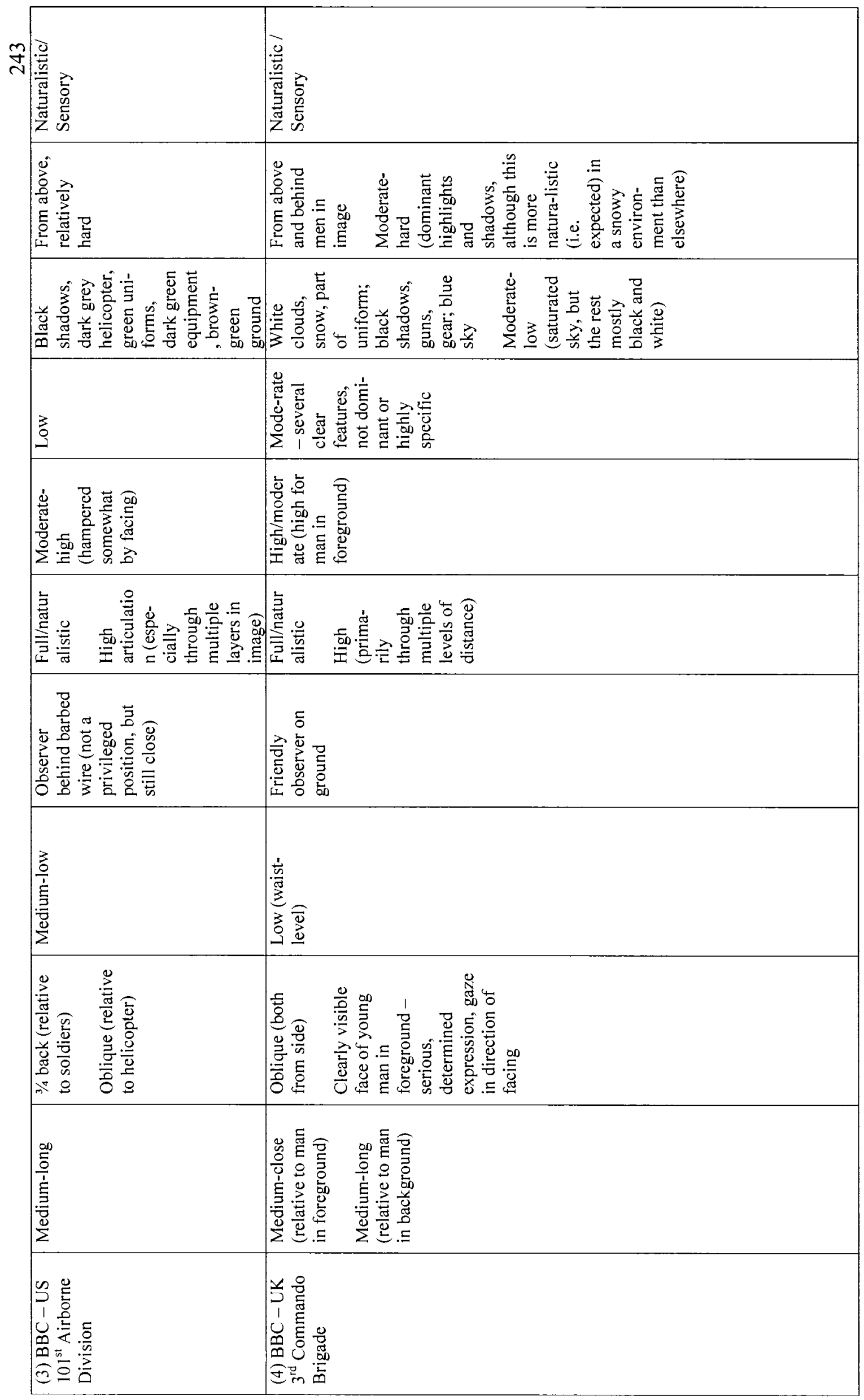




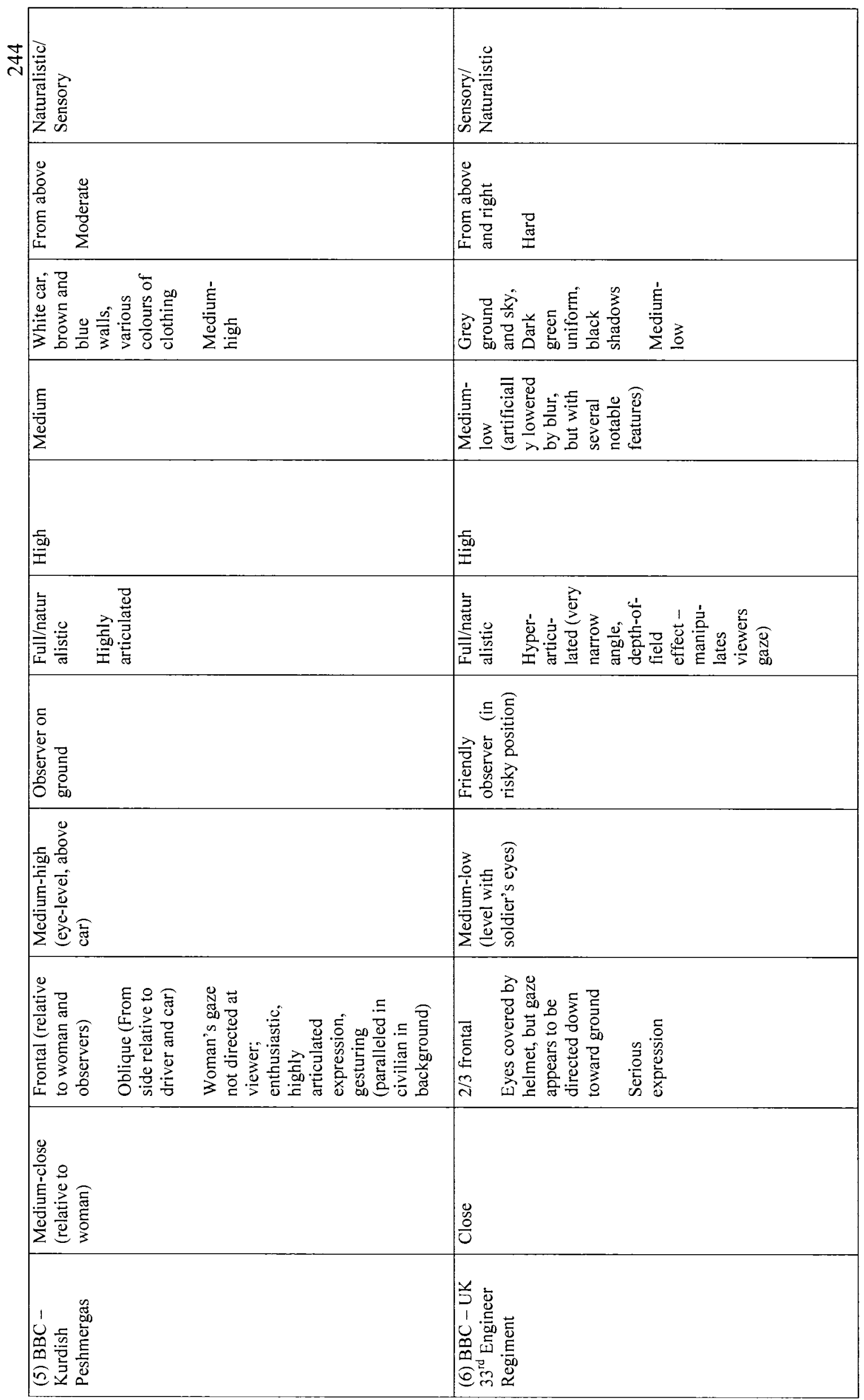




\begin{tabular}{|c|c|}
\hline 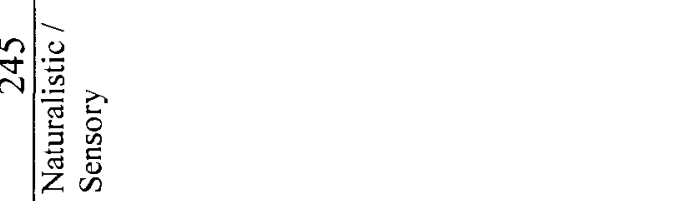 & 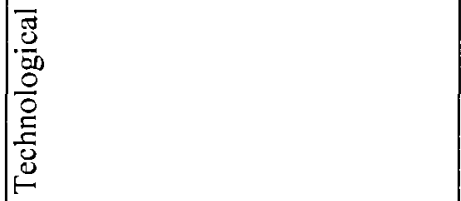 \\
\hline 䓂 & 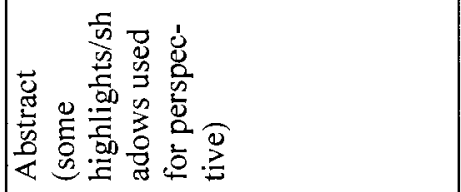 \\
\hline 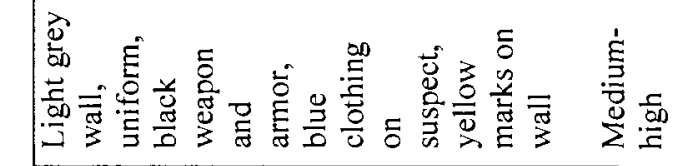 & 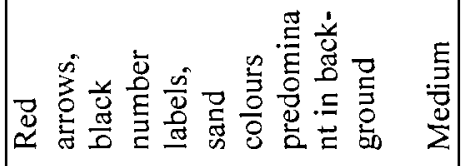 \\
\hline 染 & $\frac{3}{\frac{3}{2}}$ \\
\hline$\frac{\bar{D}}{\bar{D}}$ & 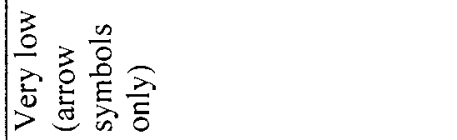 \\
\hline 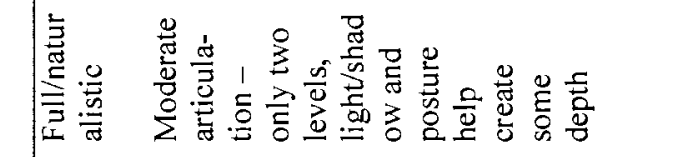 & 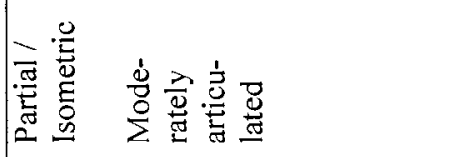 \\
\hline 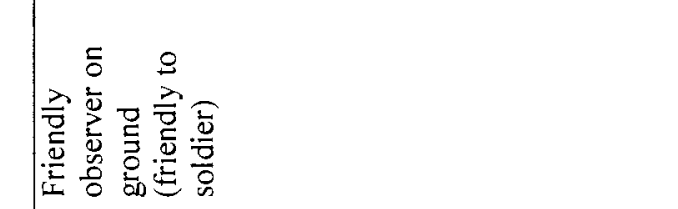 & 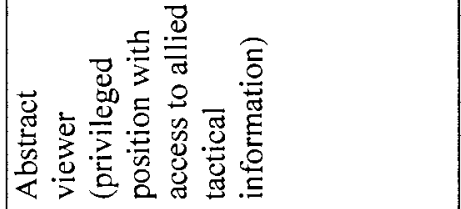 \\
\hline 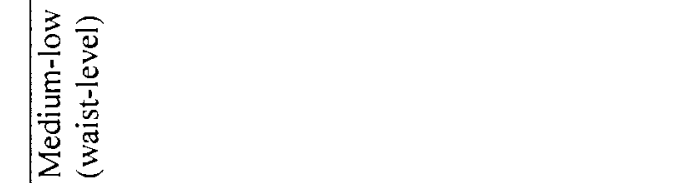 & 竞 \\
\hline 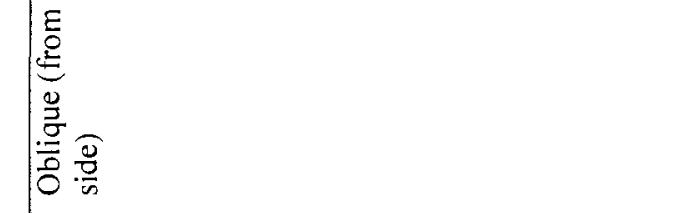 & 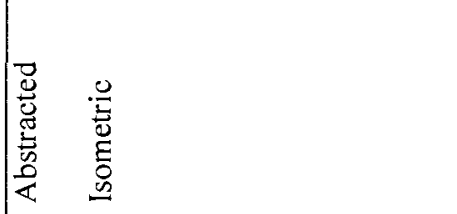 \\
\hline 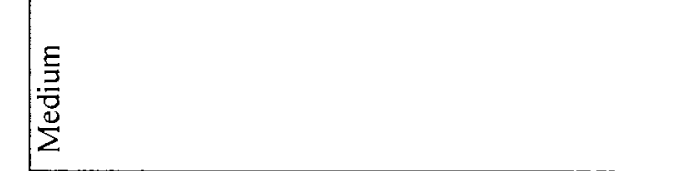 & 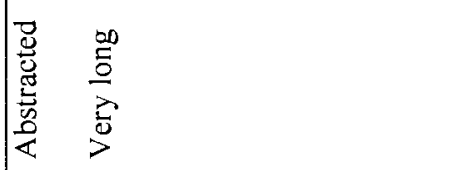 \\
\hline 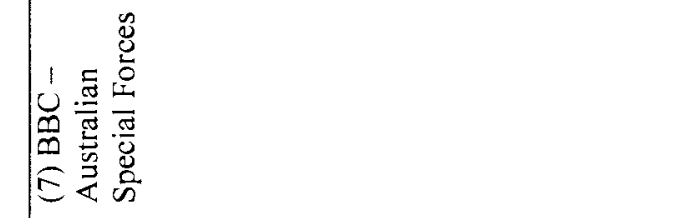 & 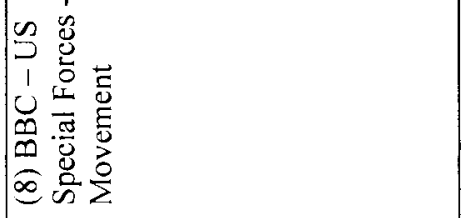 \\
\hline
\end{tabular}




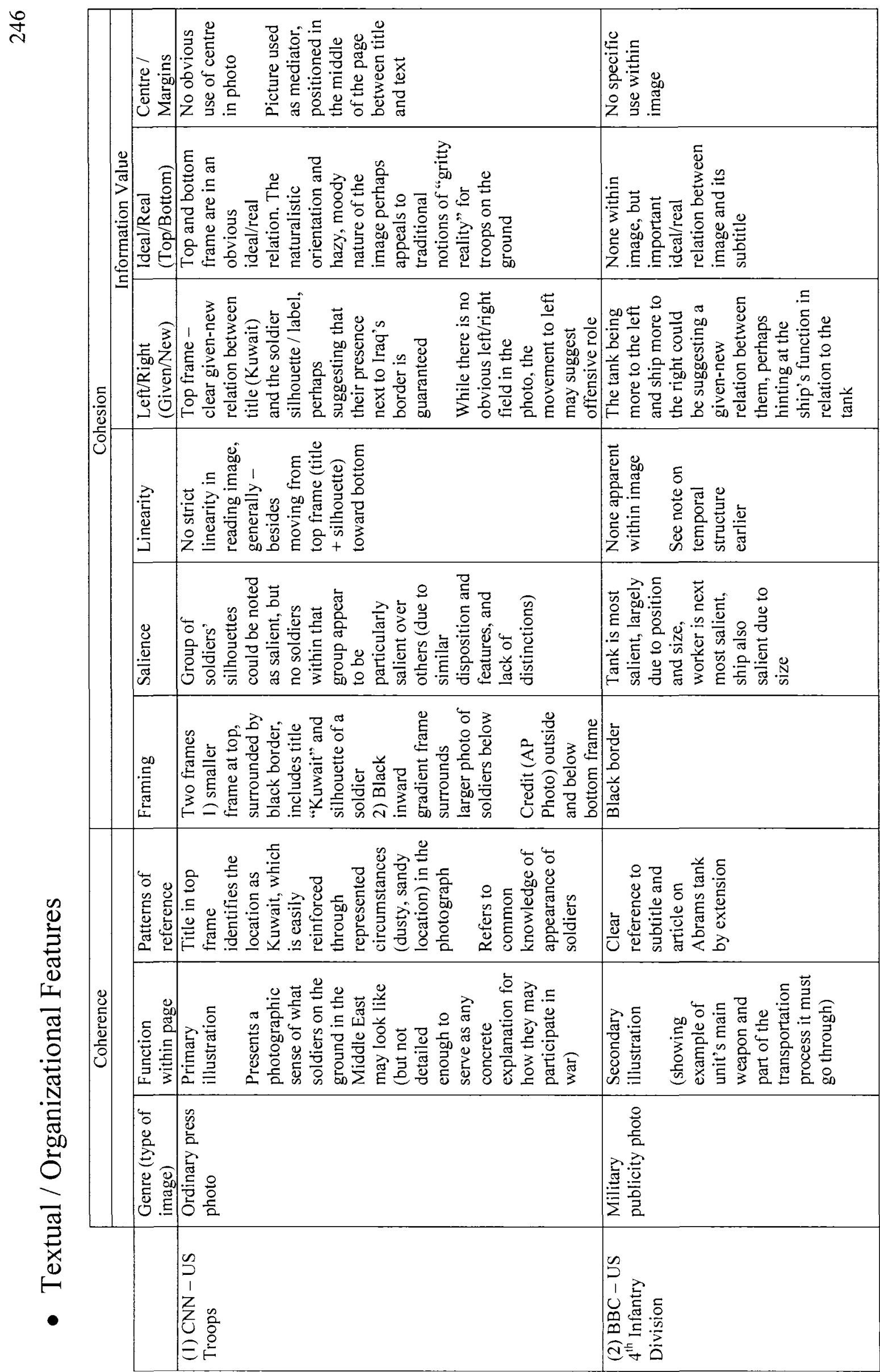




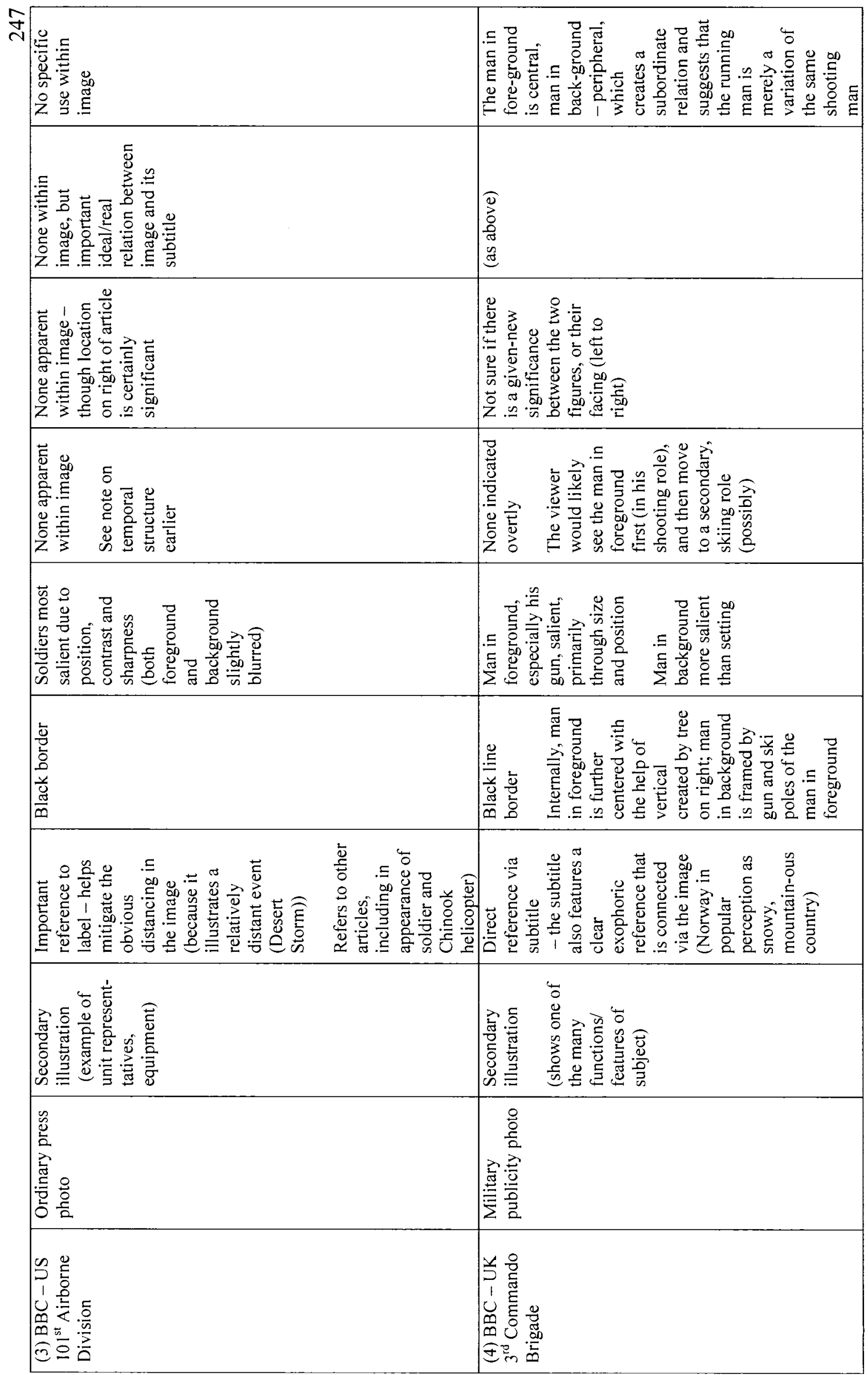




\begin{tabular}{|c|c|}
\hline 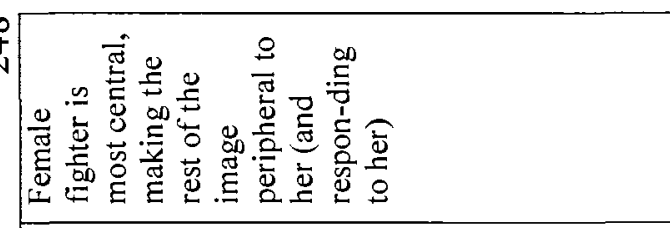 & 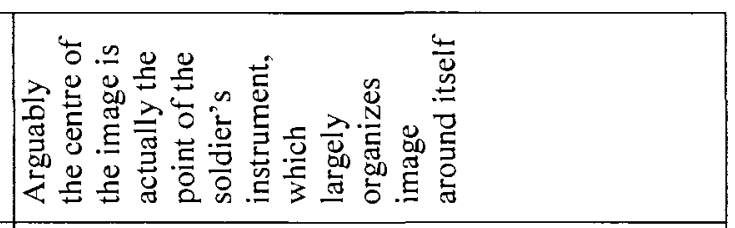 \\
\hline 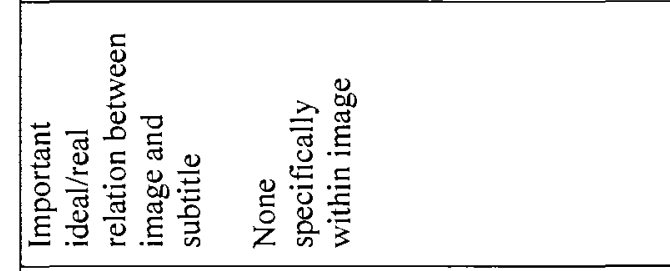 & 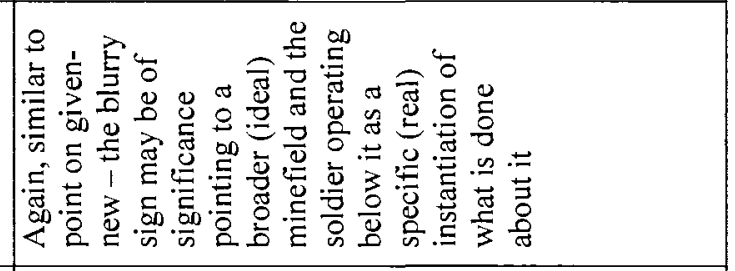 \\
\hline 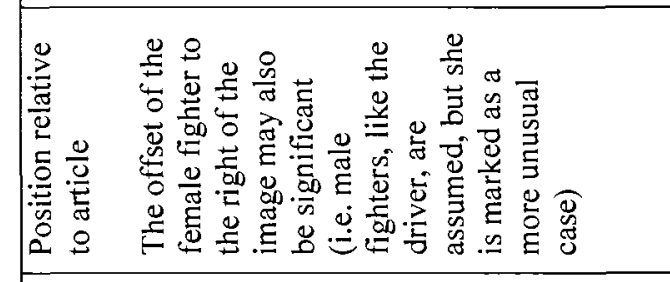 & 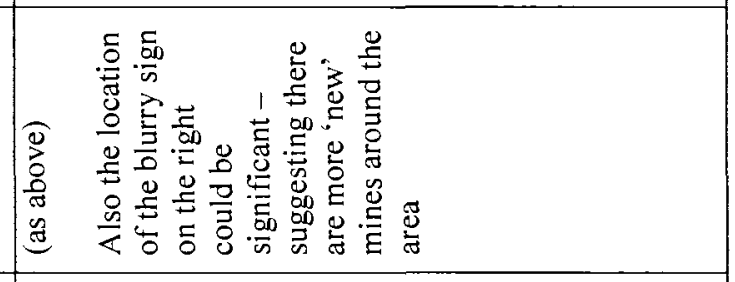 \\
\hline 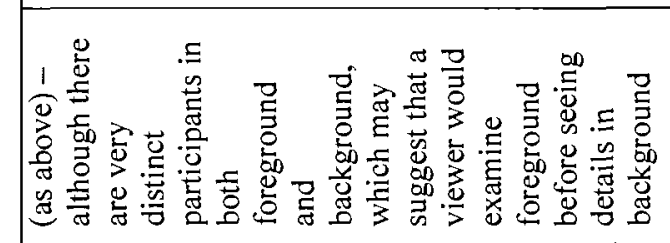 & 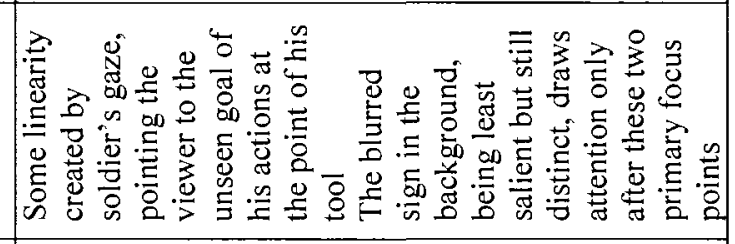 \\
\hline 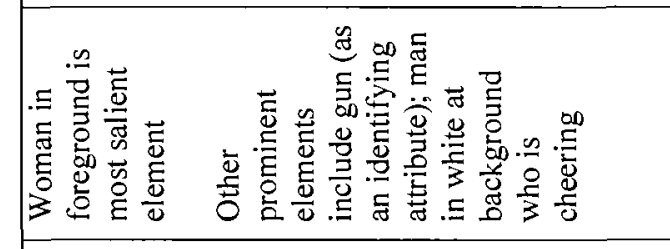 & 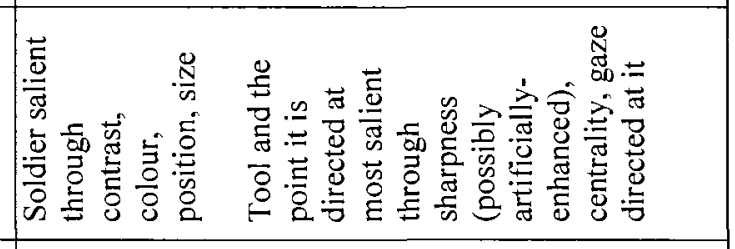 \\
\hline 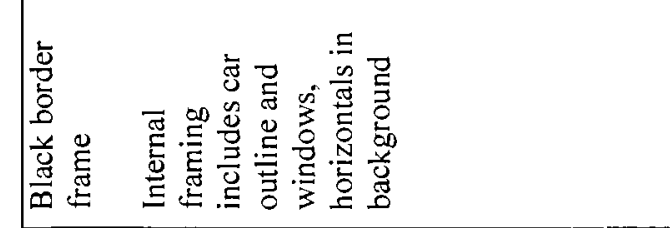 & 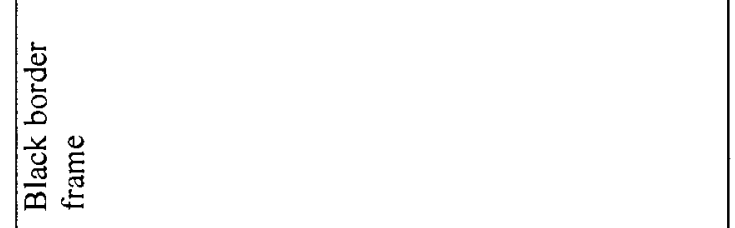 \\
\hline 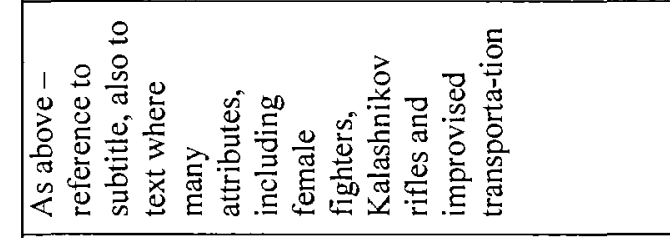 & 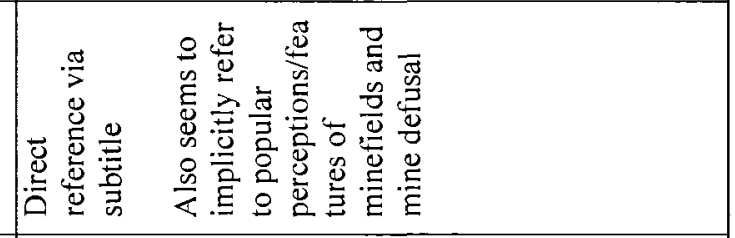 \\
\hline 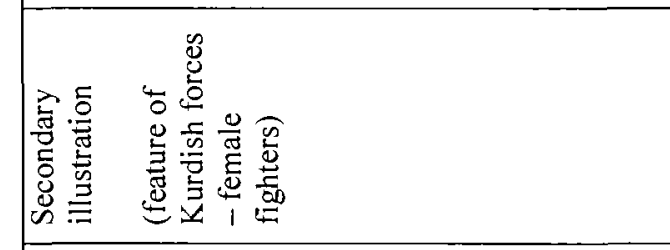 & 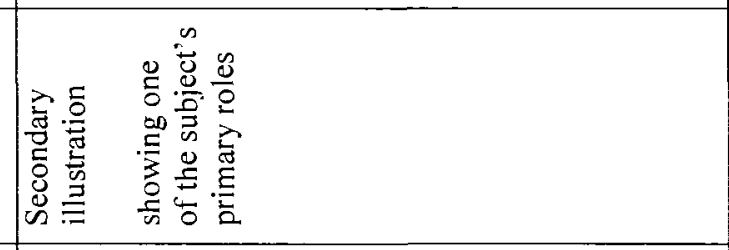 \\
\hline 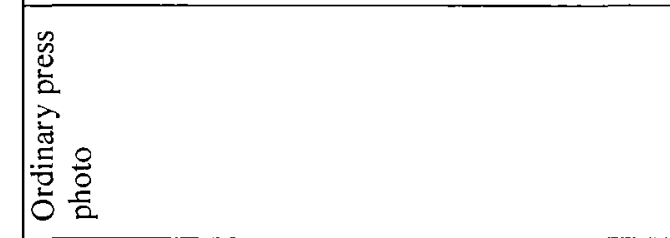 & 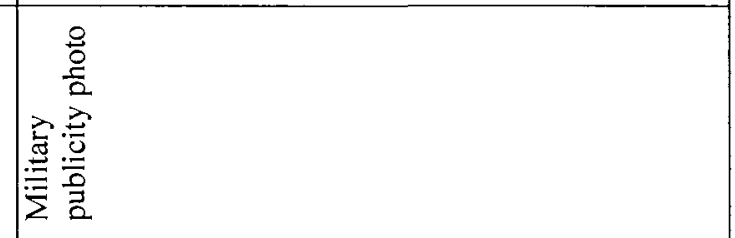 \\
\hline 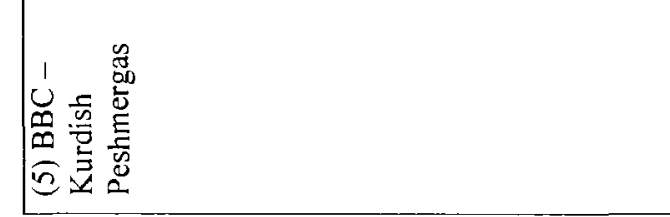 & 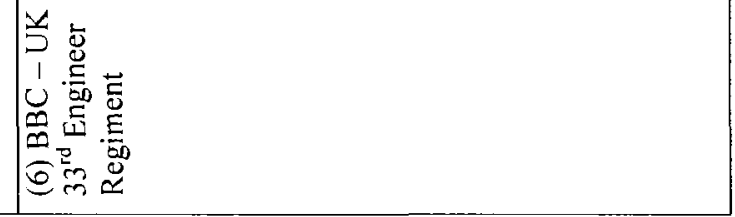 \\
\hline
\end{tabular}




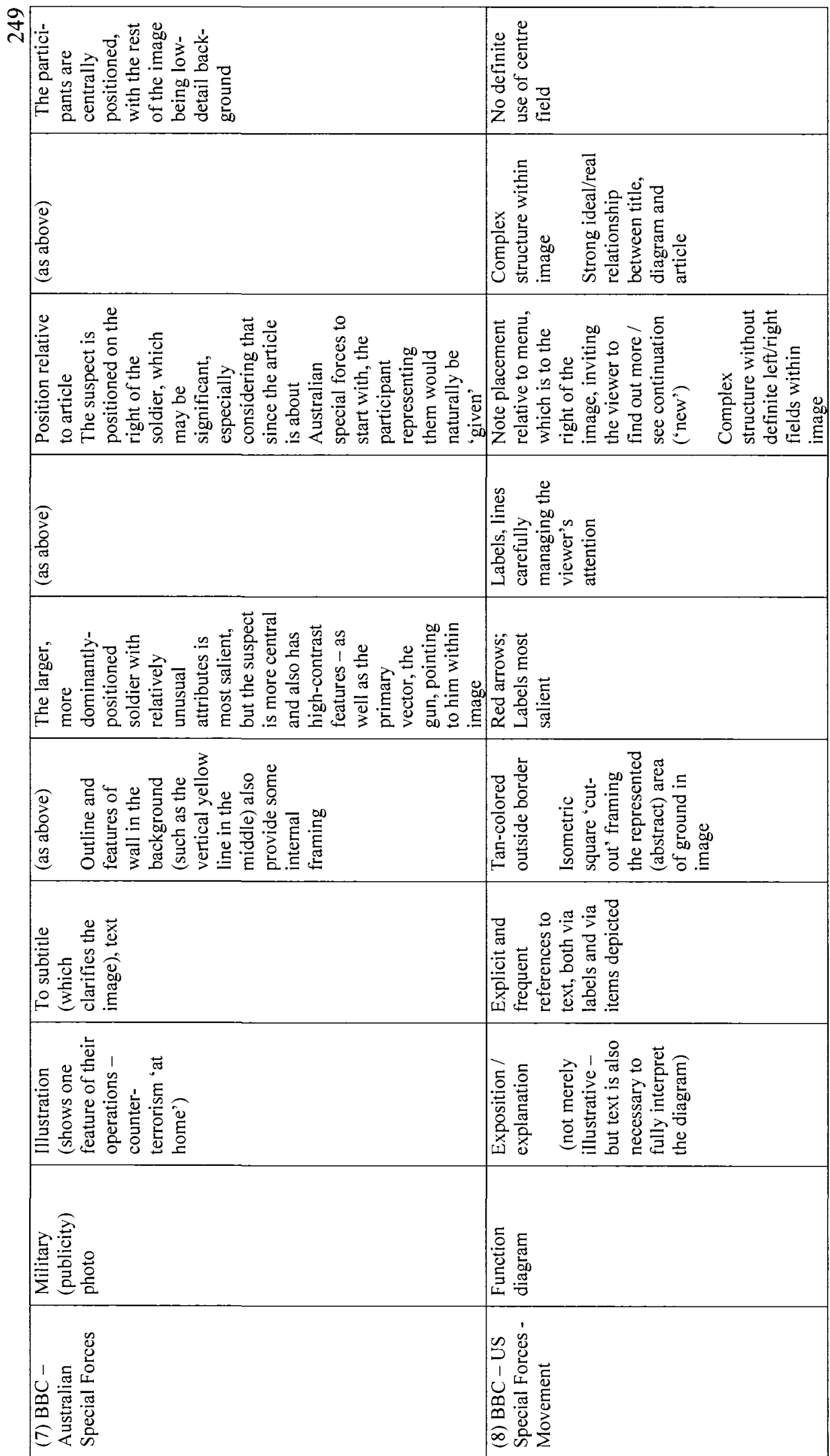




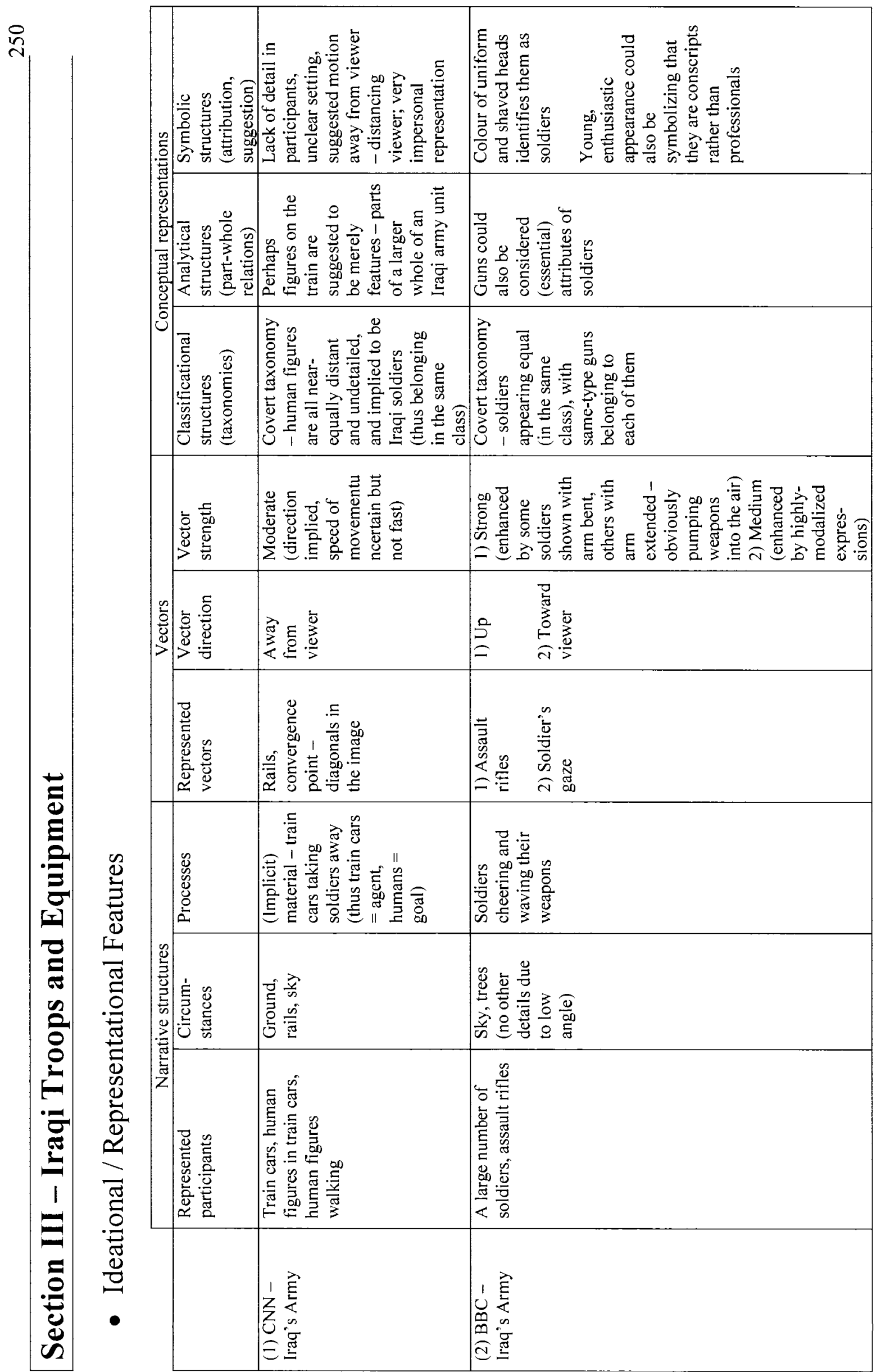




\begin{tabular}{|c|c|}
\hline 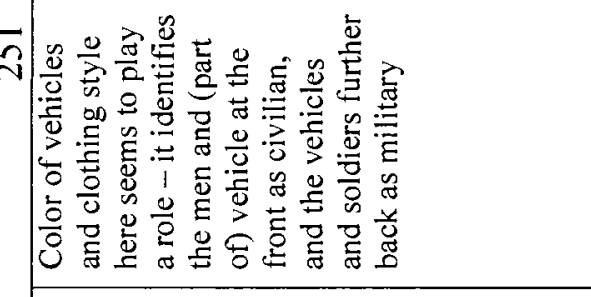 & 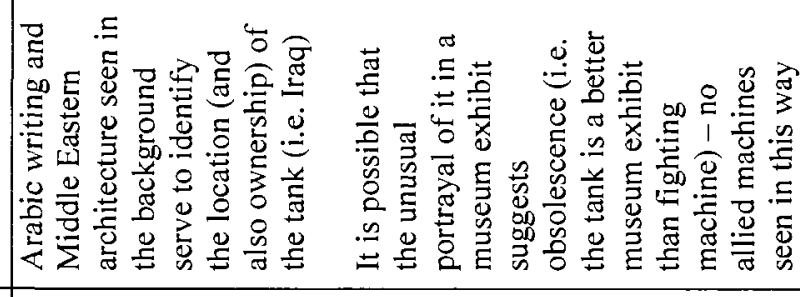 \\
\hline 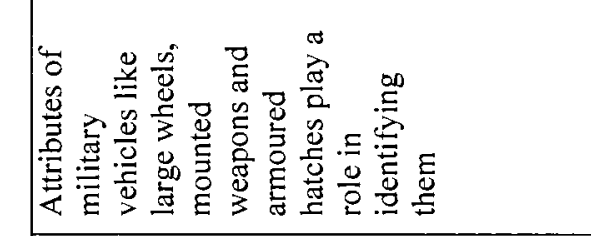 & 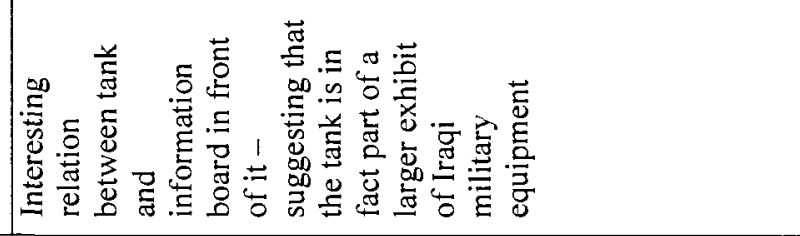 \\
\hline 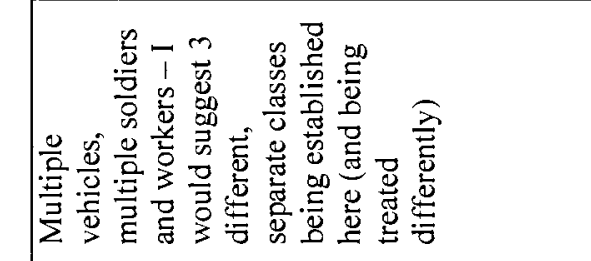 & 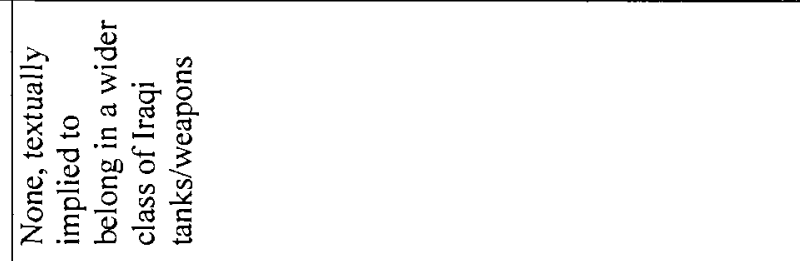 \\
\hline 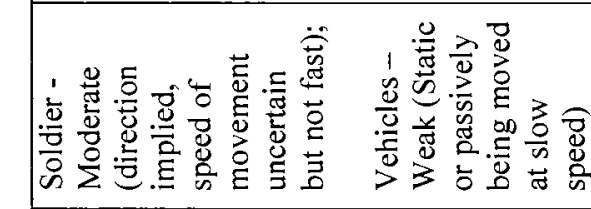 & 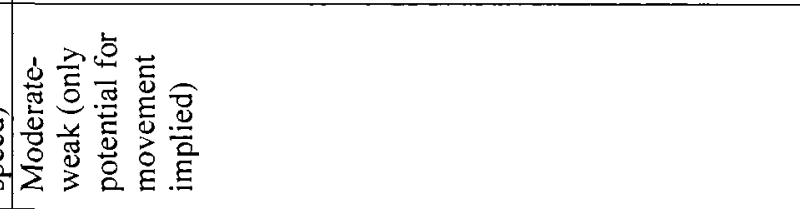 \\
\hline 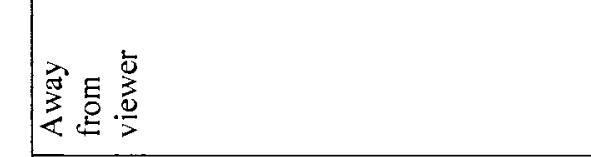 & 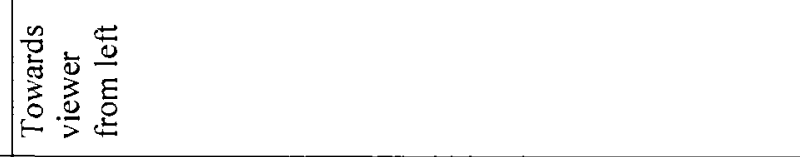 \\
\hline 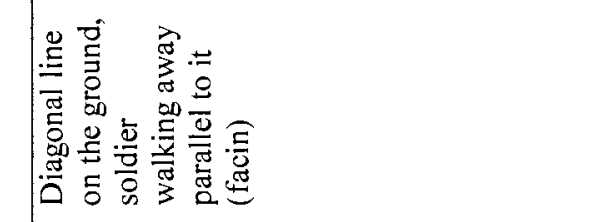 & 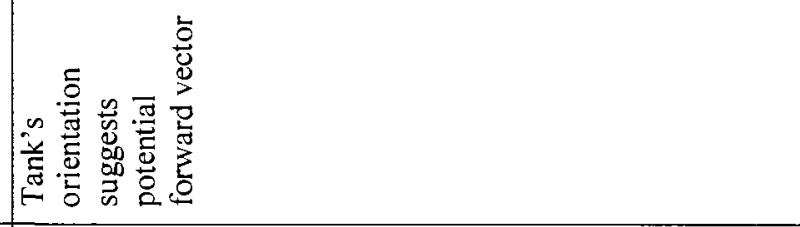 \\
\hline 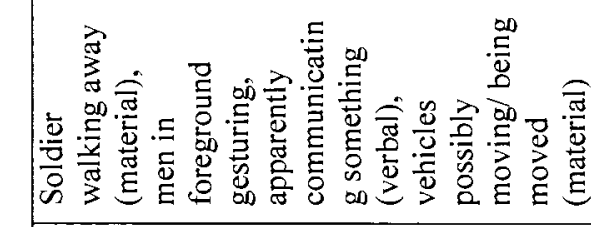 & 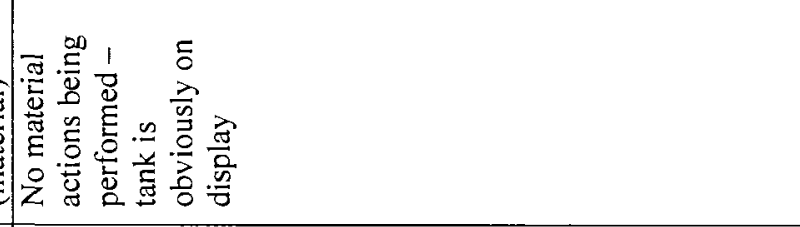 \\
\hline 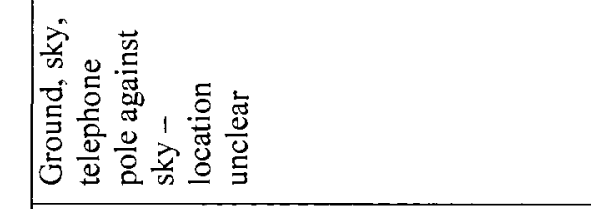 & 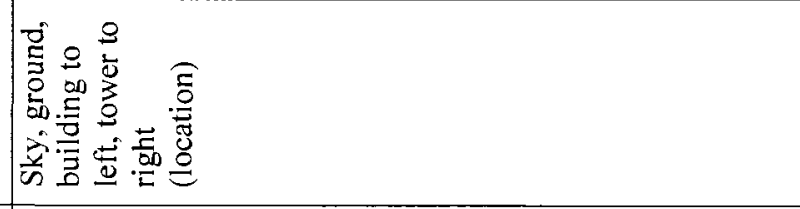 \\
\hline 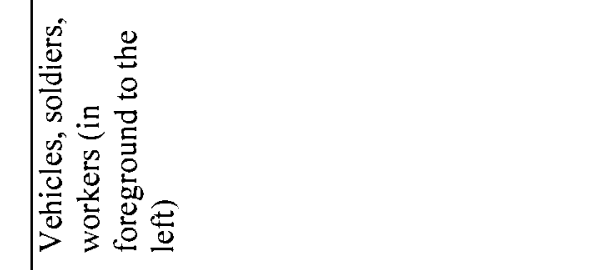 & 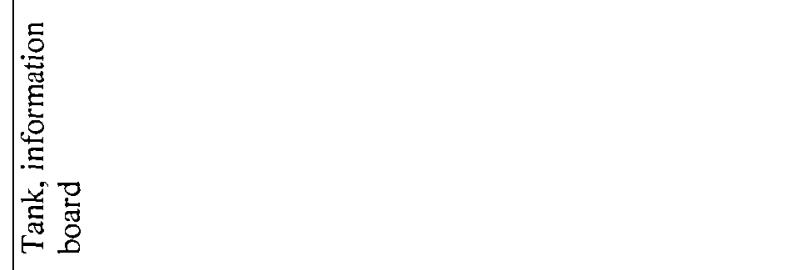 \\
\hline 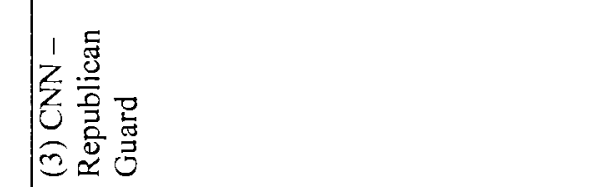 & 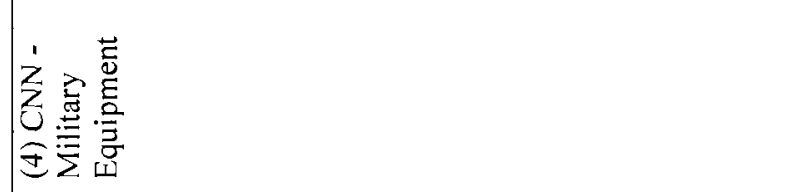 \\
\hline
\end{tabular}




\begin{tabular}{|c|c|}
\hline 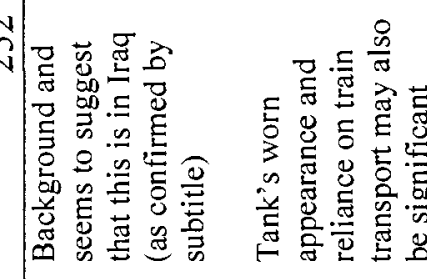 & 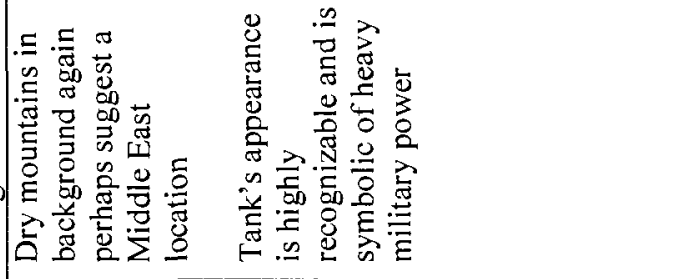 \\
\hline & 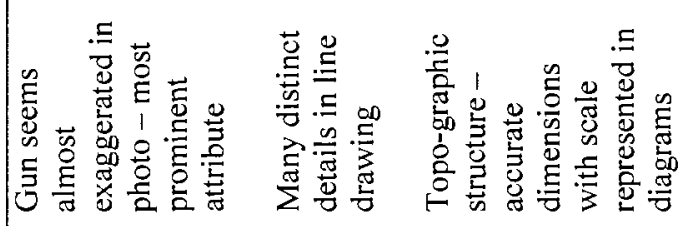 \\
\hline 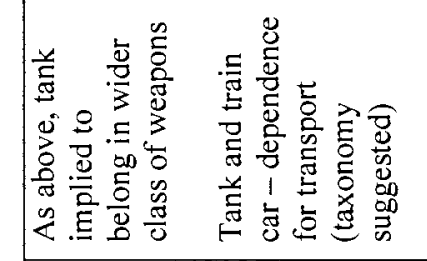 & 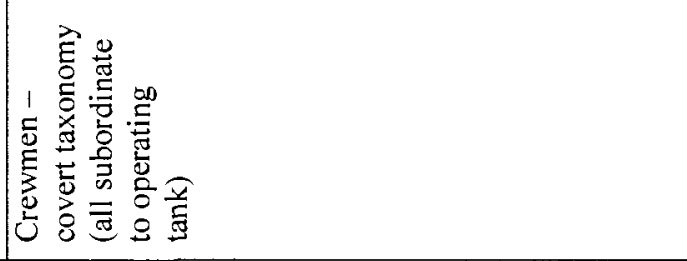 \\
\hline 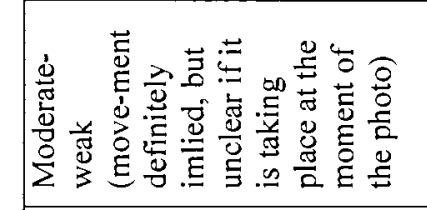 & 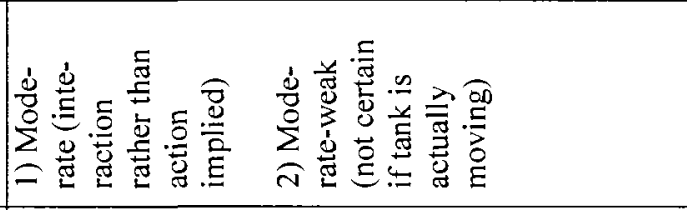 \\
\hline 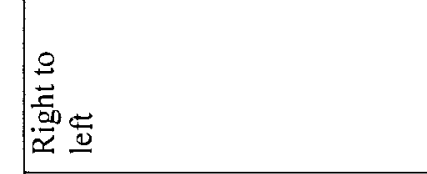 & 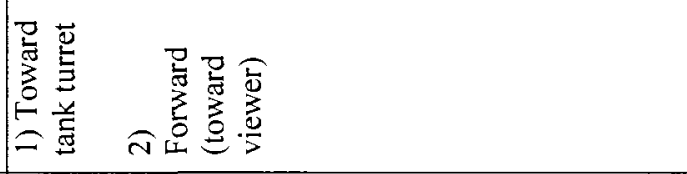 \\
\hline 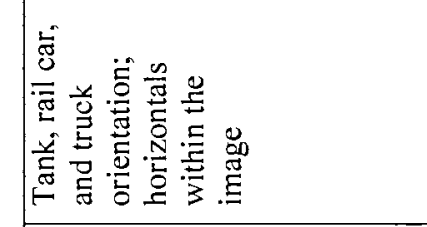 & 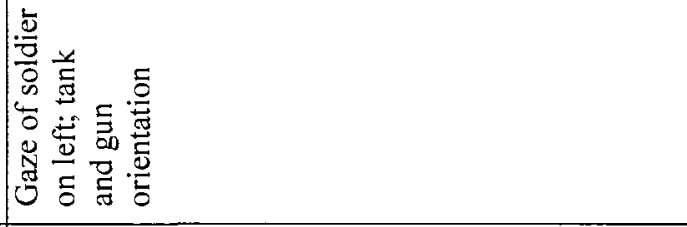 \\
\hline 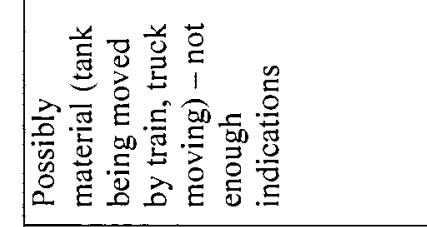 & 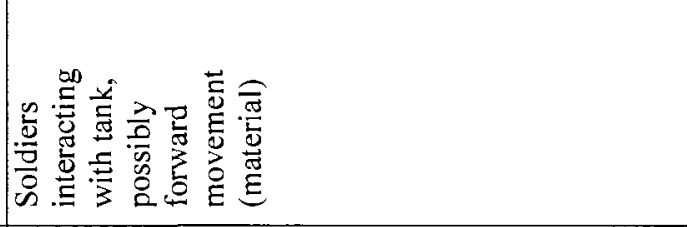 \\
\hline 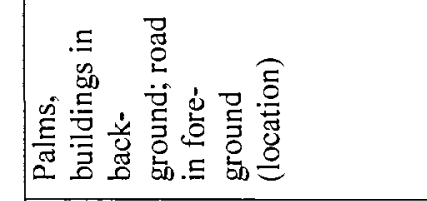 & 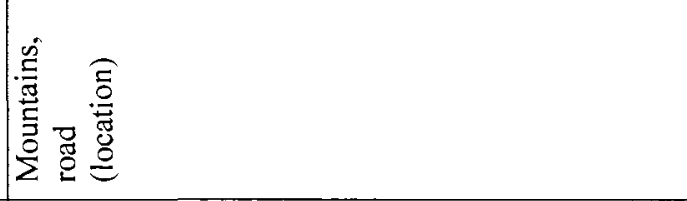 \\
\hline 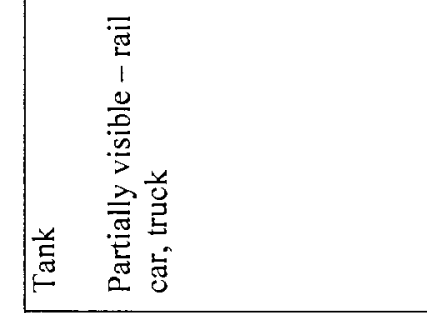 & 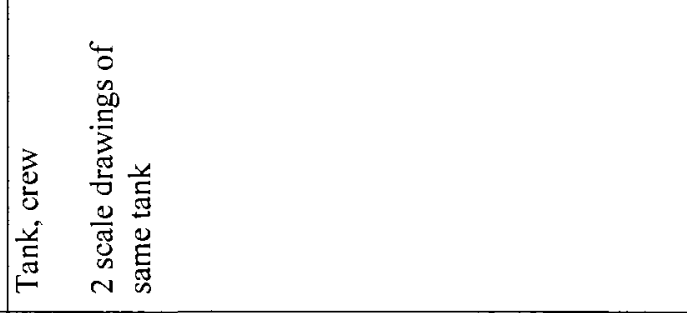 \\
\hline 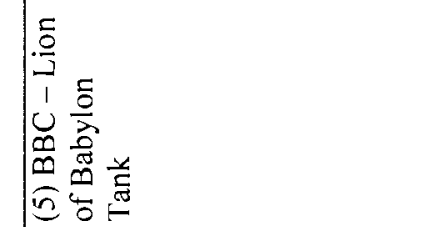 & 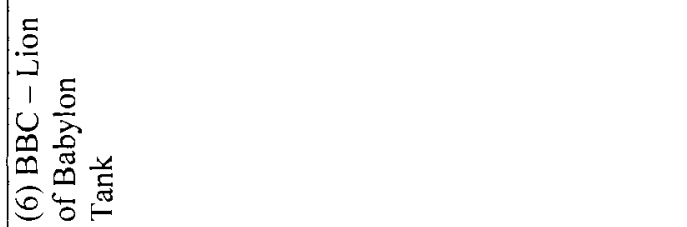 \\
\hline
\end{tabular}




\begin{tabular}{|c|c|}
\hline 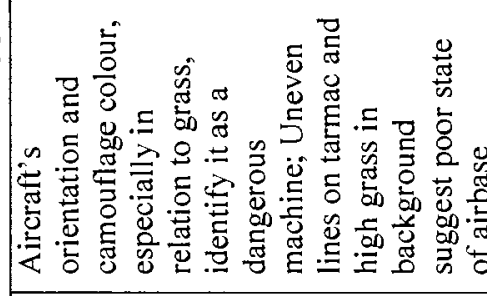 & 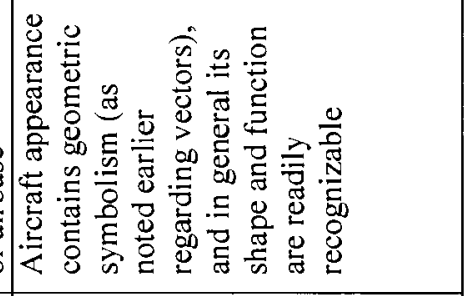 \\
\hline 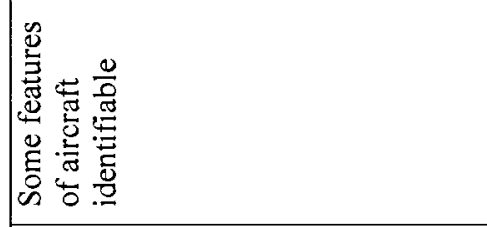 & 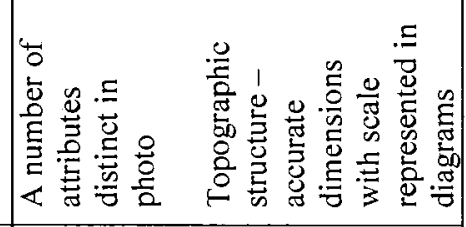 \\
\hline 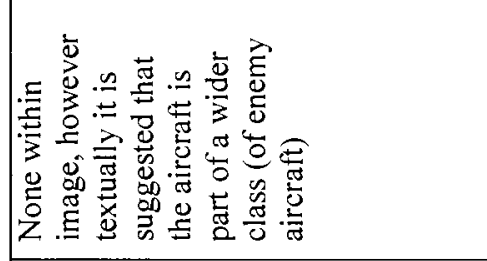 & 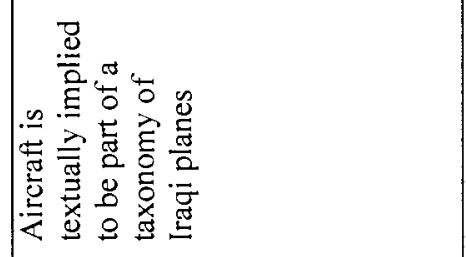 \\
\hline 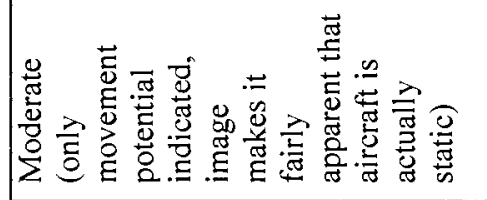 & 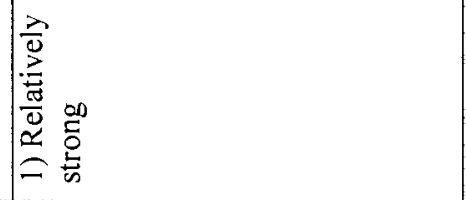 \\
\hline 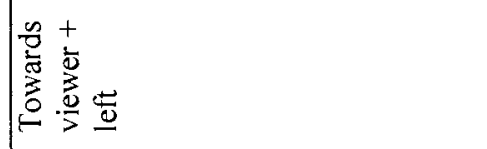 & 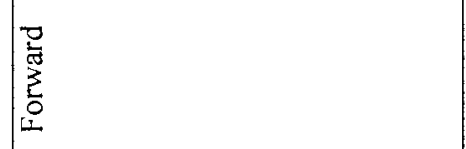 \\
\hline 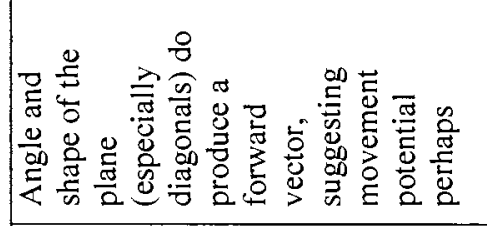 & 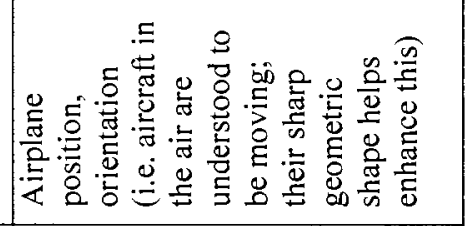 \\
\hline 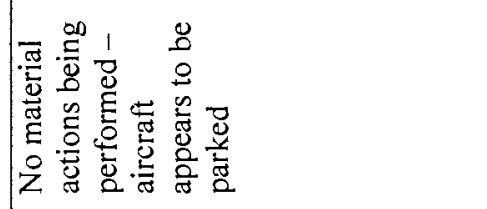 & 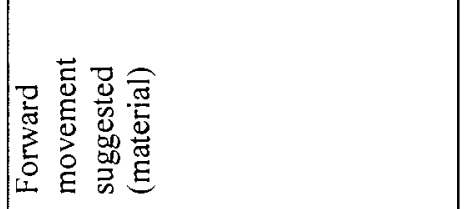 \\
\hline 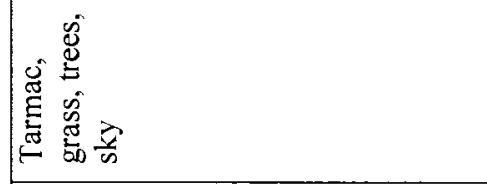 & 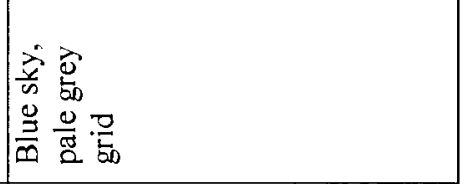 \\
\hline 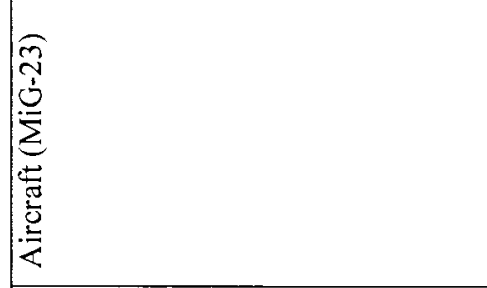 & 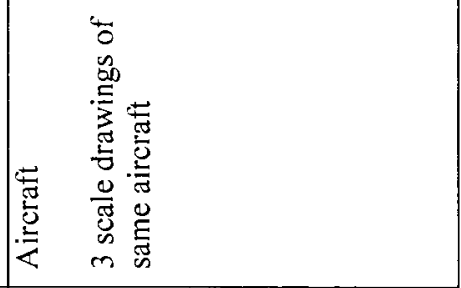 \\
\hline 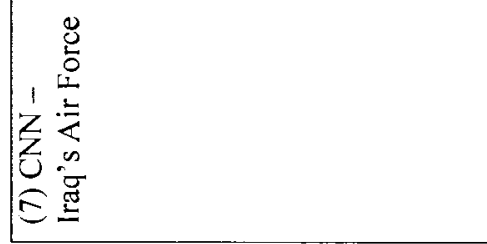 & 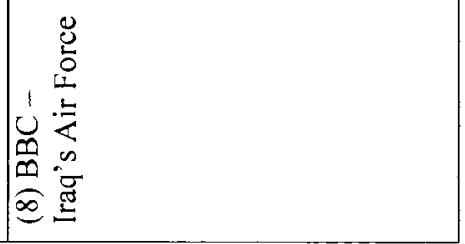 \\
\hline
\end{tabular}




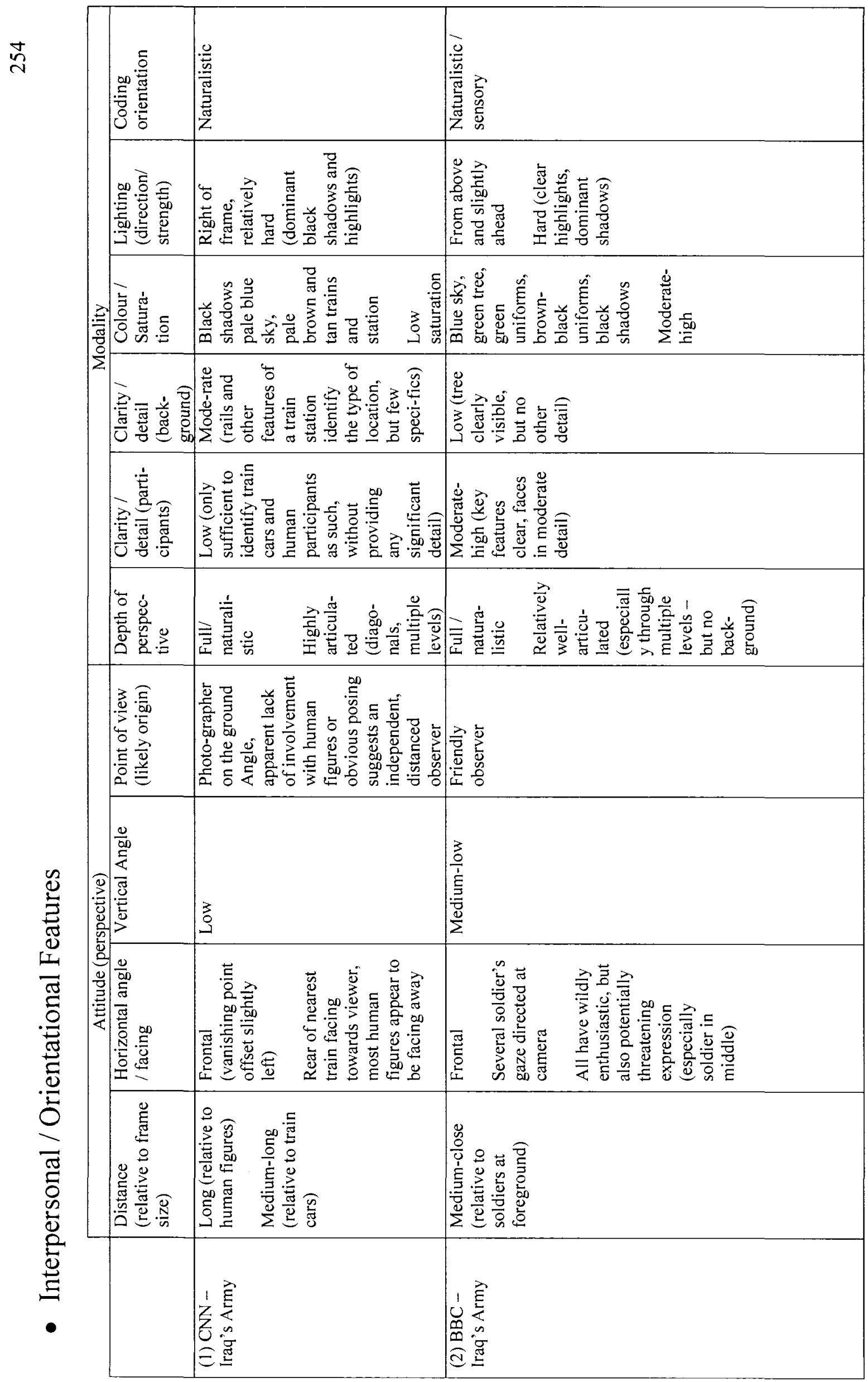




\begin{tabular}{|c|c|}
\hline & 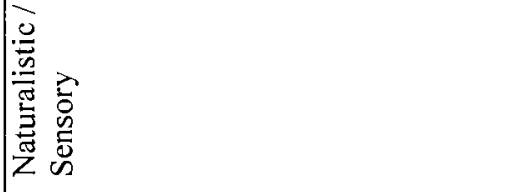 \\
\hline 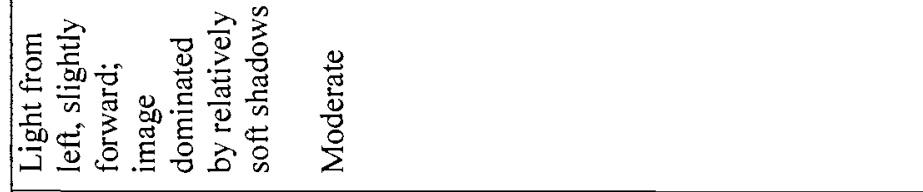 & 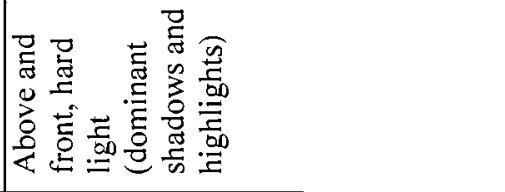 \\
\hline 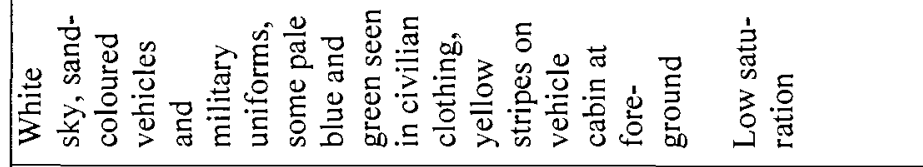 & 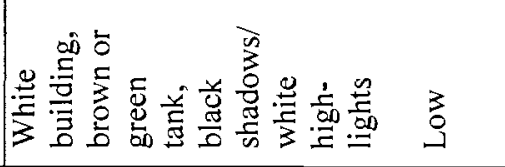 \\
\hline 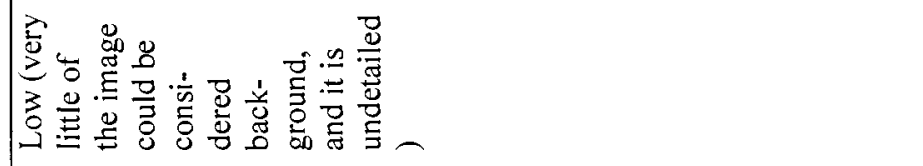 & 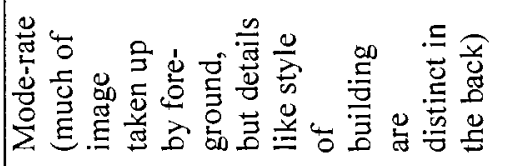 \\
\hline 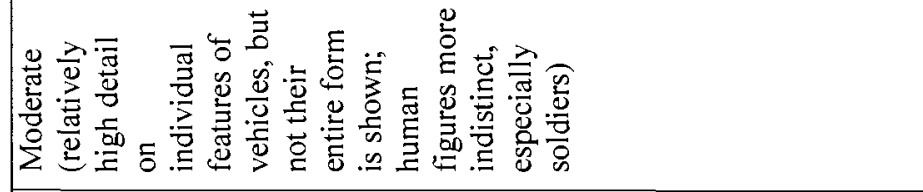 & 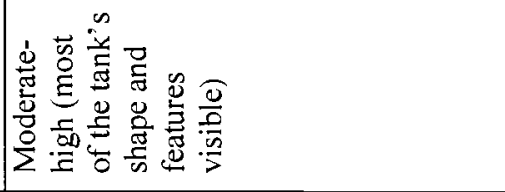 \\
\hline 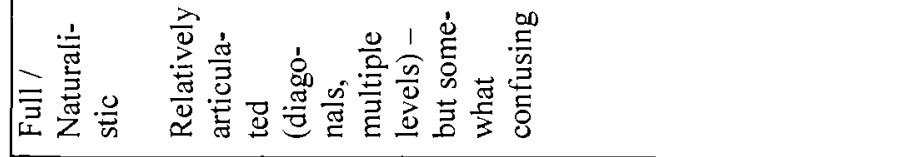 & 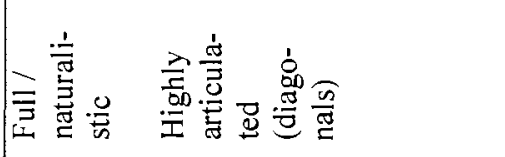 \\
\hline 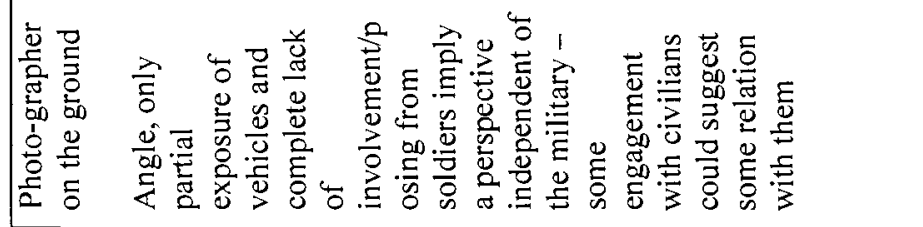 & 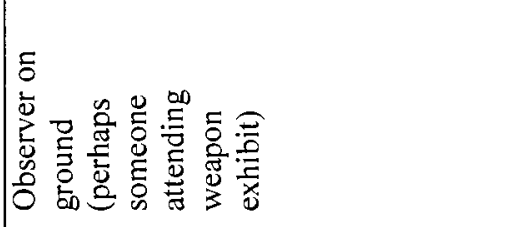 \\
\hline 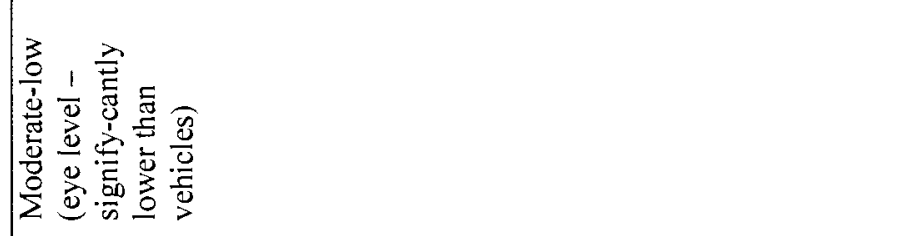 & 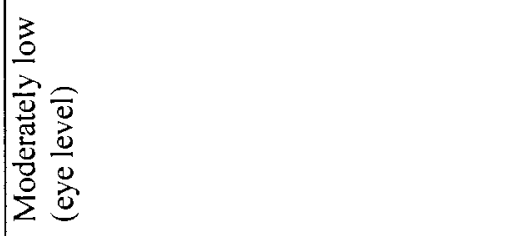 \\
\hline 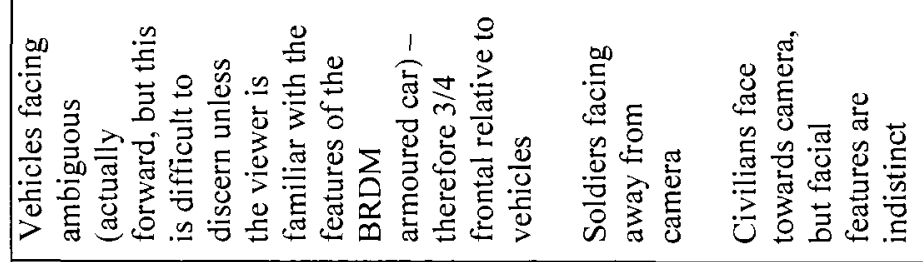 & 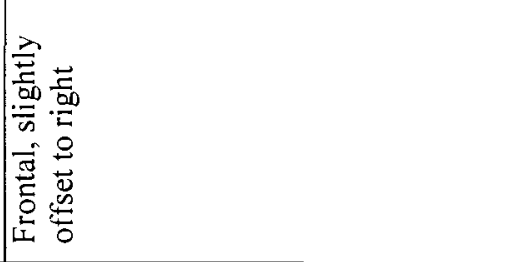 \\
\hline 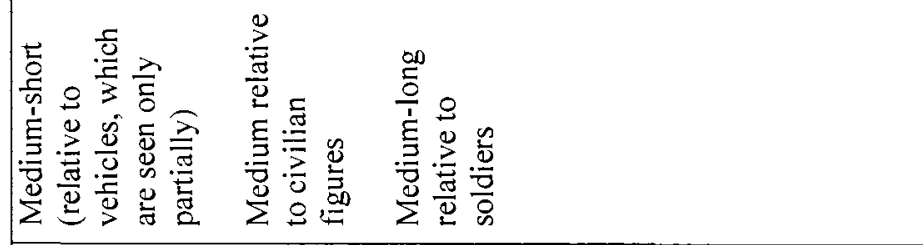 & 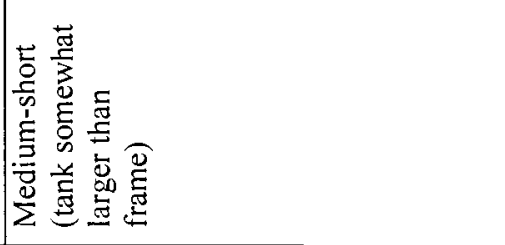 \\
\hline 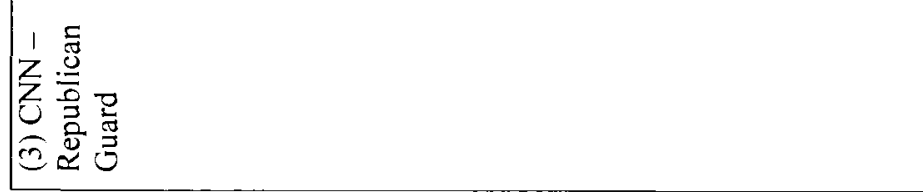 & 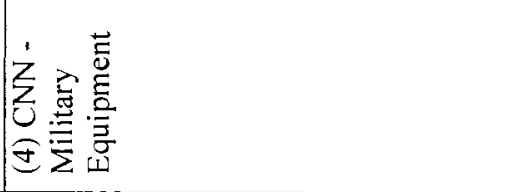 \\
\hline
\end{tabular}




\begin{tabular}{|c|c|}
\hline ה: & 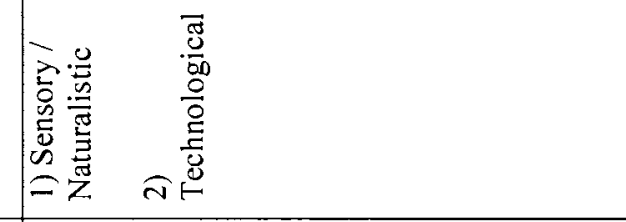 \\
\hline 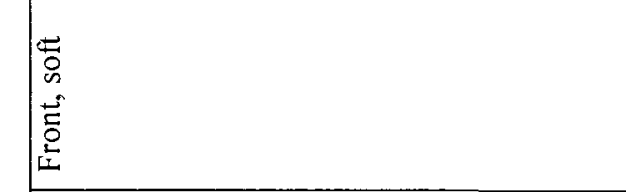 & 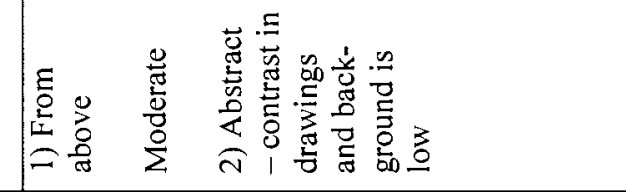 \\
\hline 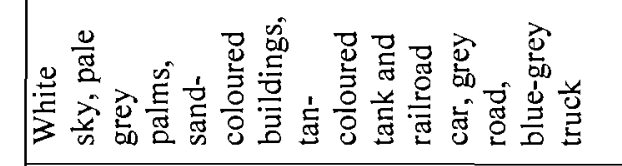 & 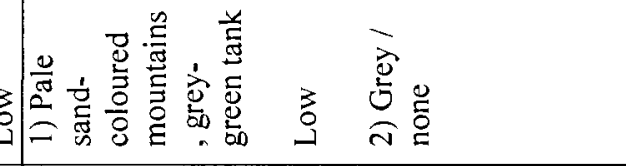 \\
\hline $\mid \frac{5}{.00}$ & 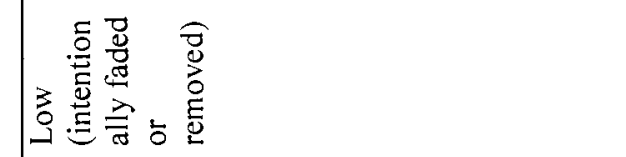 \\
\hline 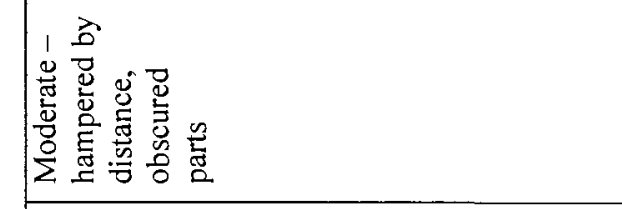 & 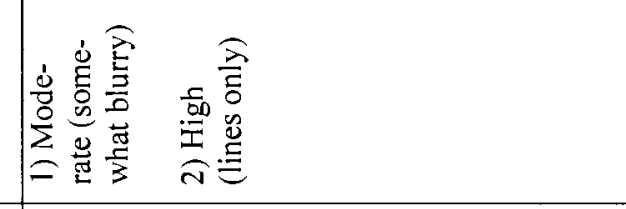 \\
\hline 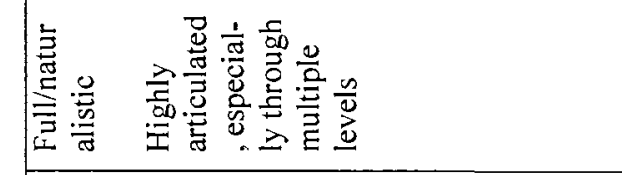 & 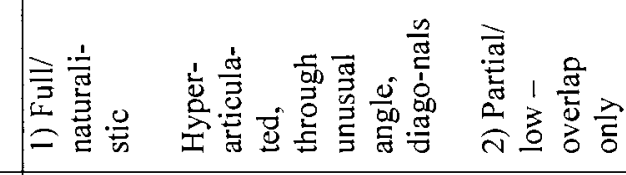 \\
\hline 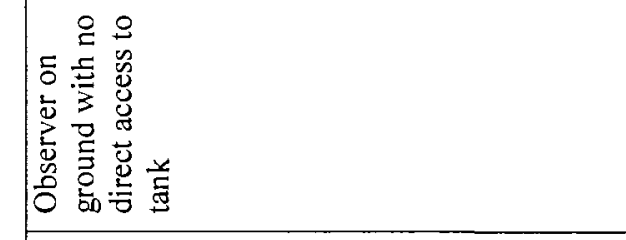 & 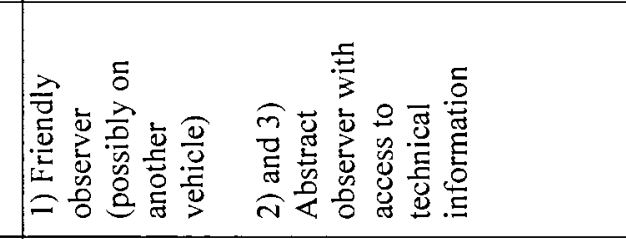 \\
\hline 䛒 & 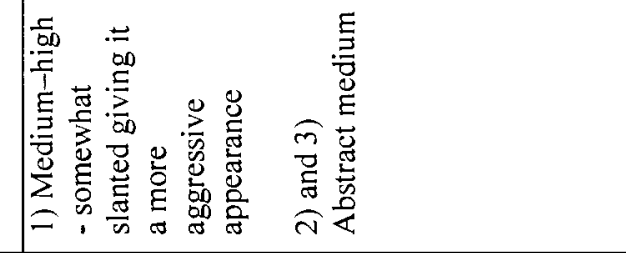 \\
\hline 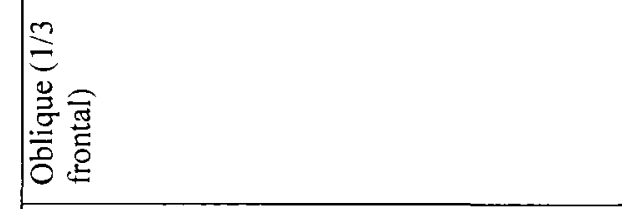 & 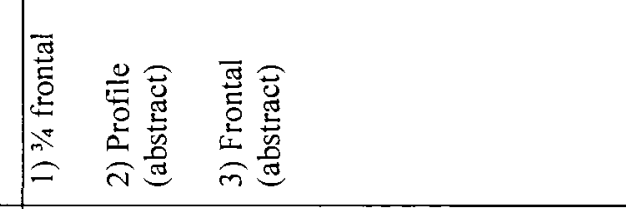 \\
\hline 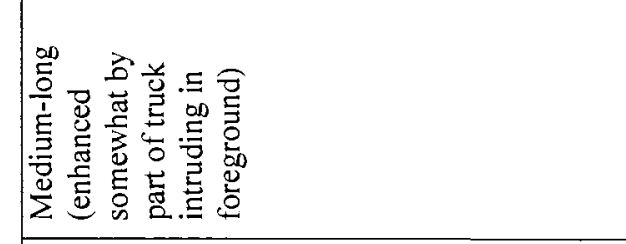 & 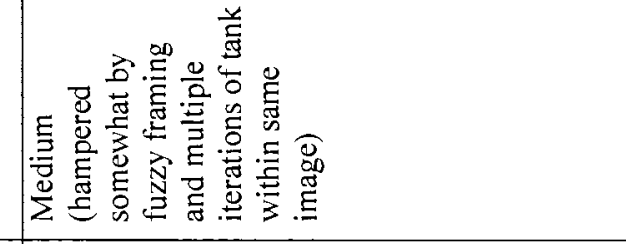 \\
\hline 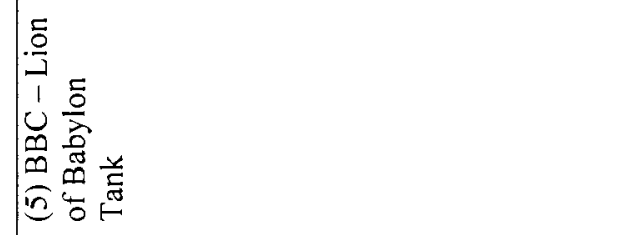 & 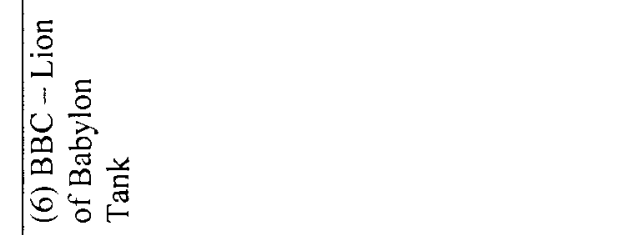 \\
\hline
\end{tabular}




\begin{tabular}{|c|c|}
\hline 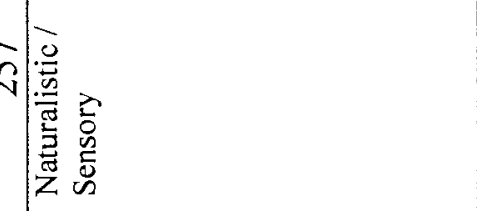 & 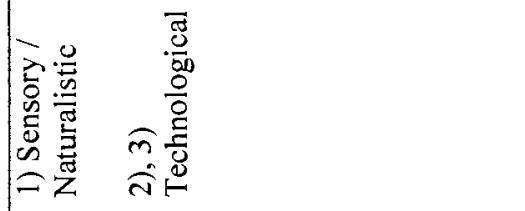 \\
\hline 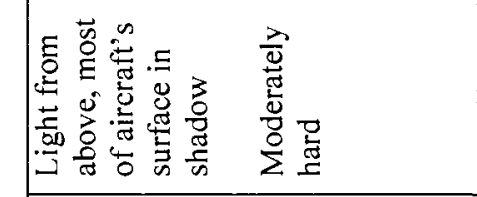 & 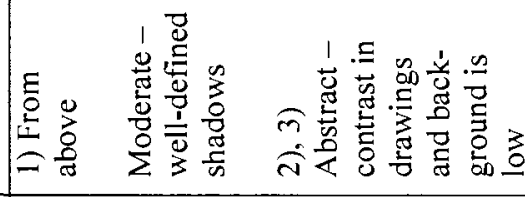 \\
\hline 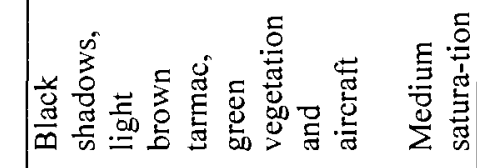 & 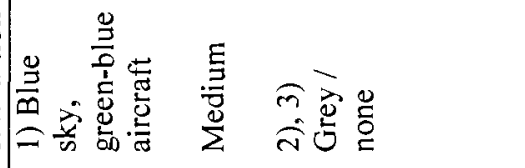 \\
\hline 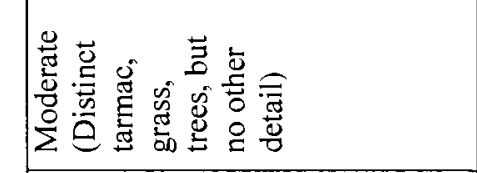 & 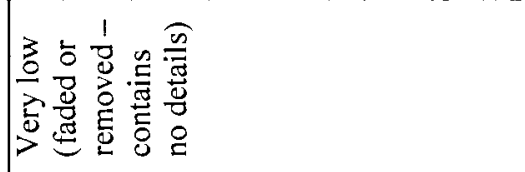 \\
\hline 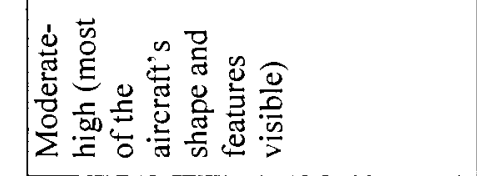 & 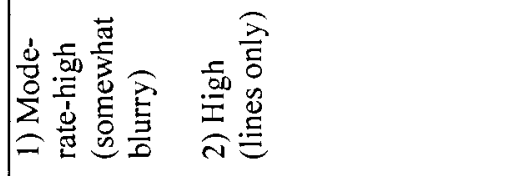 \\
\hline 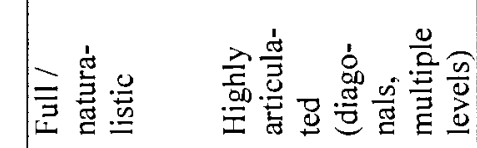 & 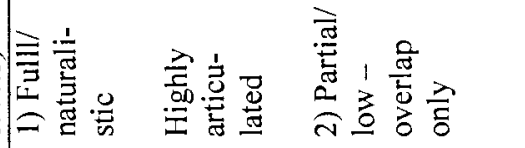 \\
\hline 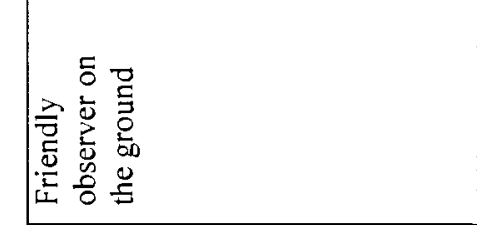 & 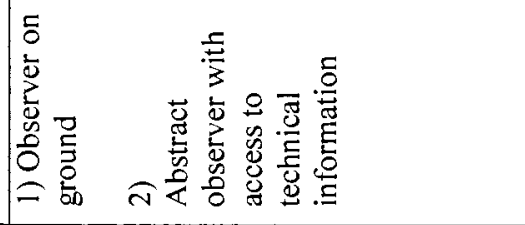 \\
\hline 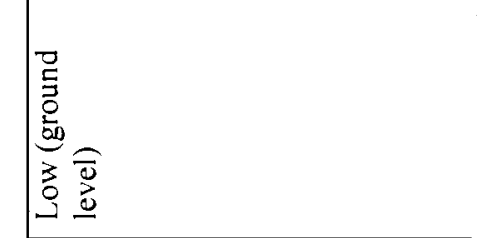 & 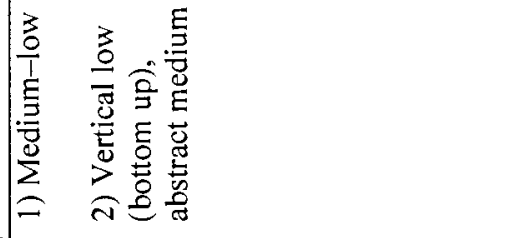 \\
\hline 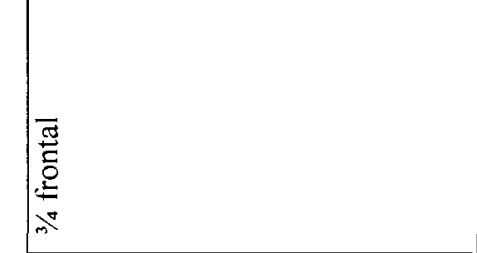 & 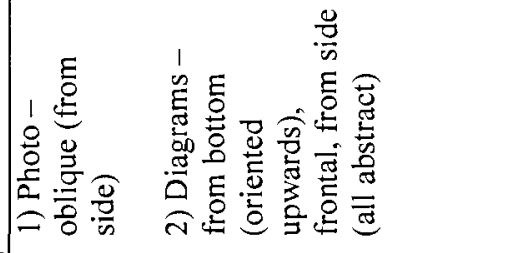 \\
\hline 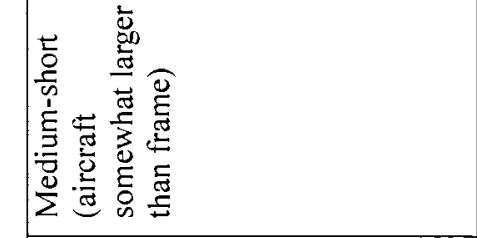 & 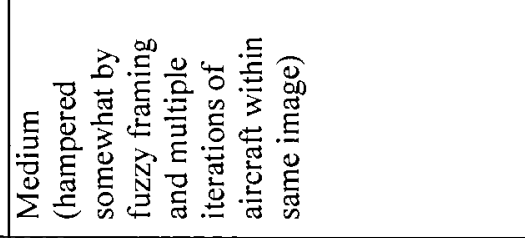 \\
\hline 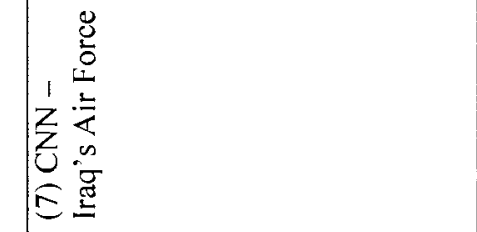 & 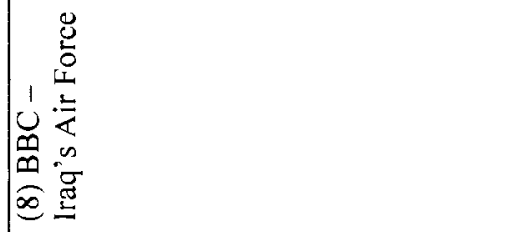 \\
\hline
\end{tabular}




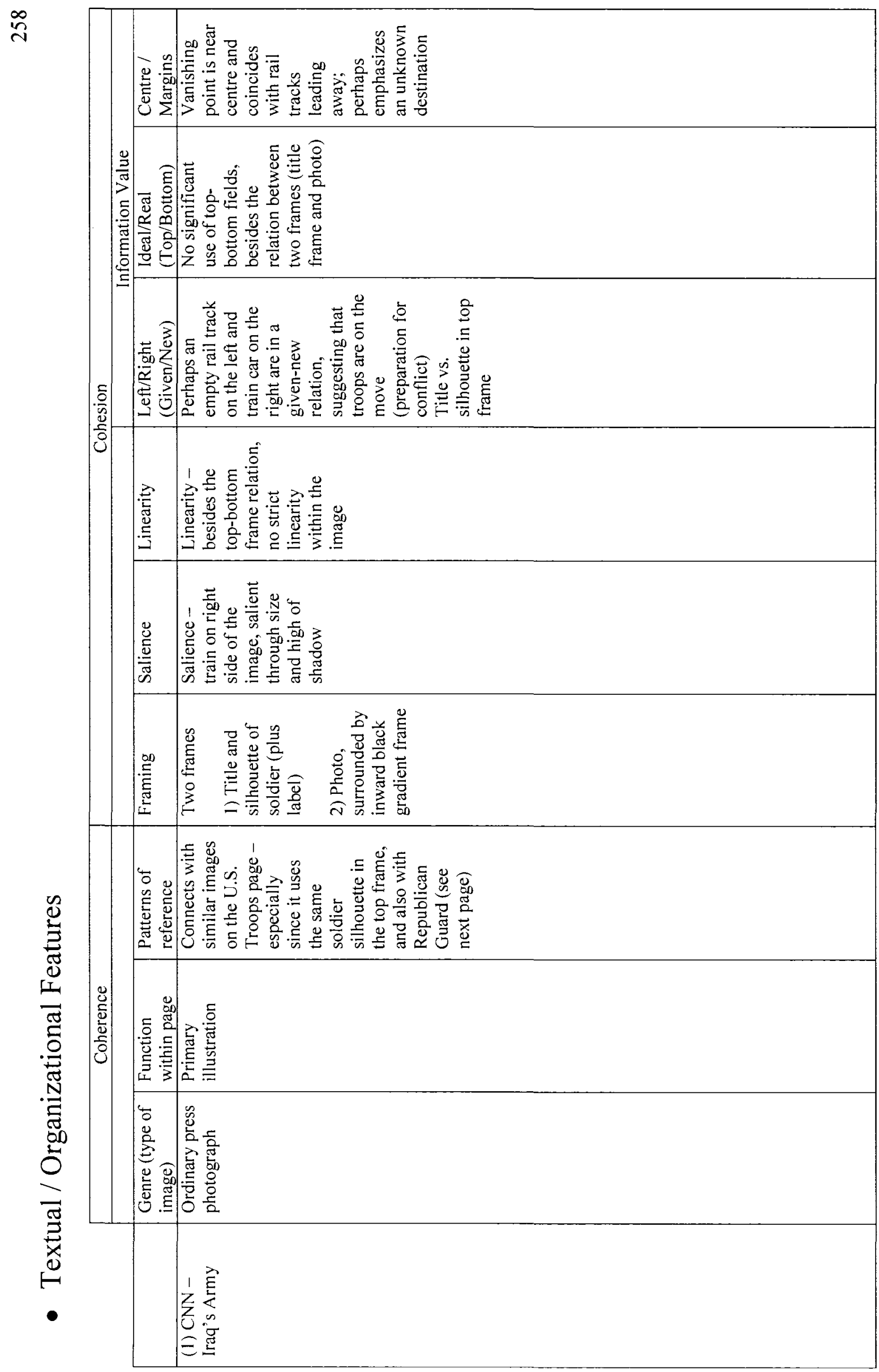




\begin{tabular}{|c|c|}
\hline 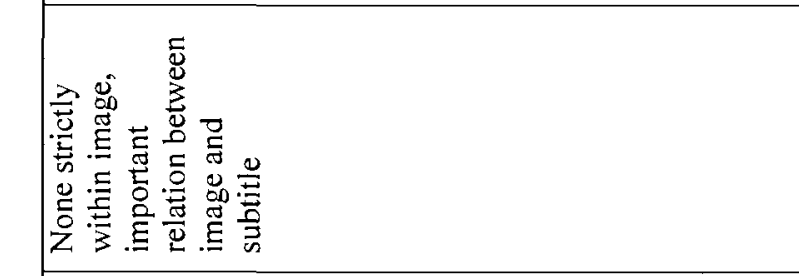 & 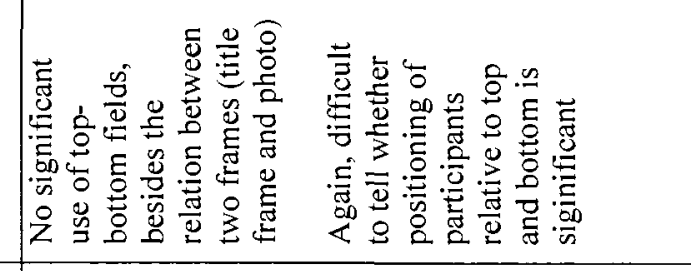 \\
\hline 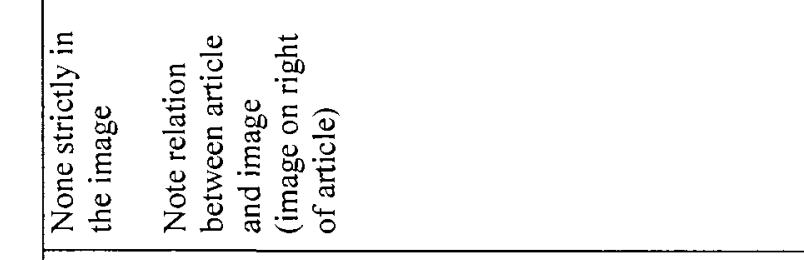 & 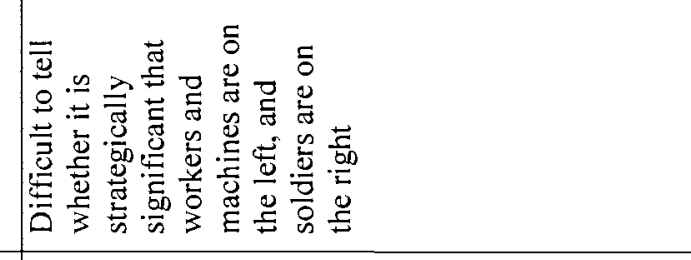 \\
\hline 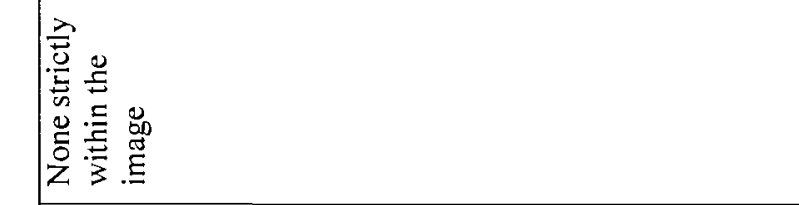 & 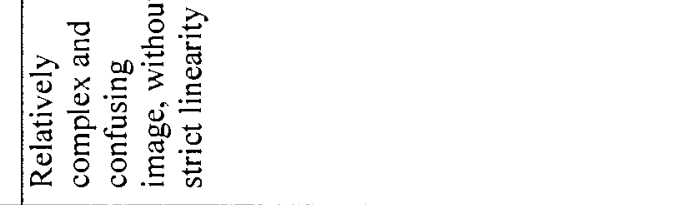 \\
\hline 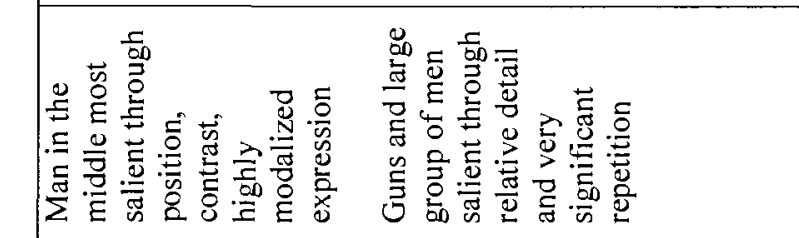 & 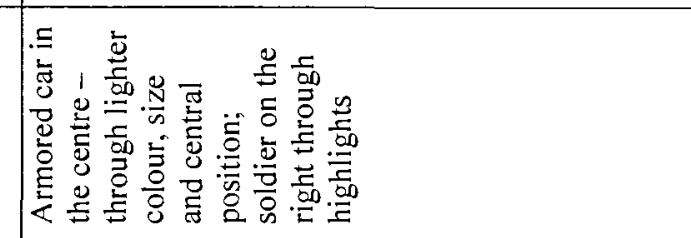 \\
\hline 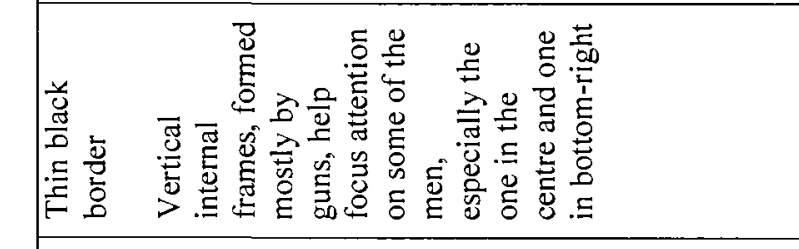 & 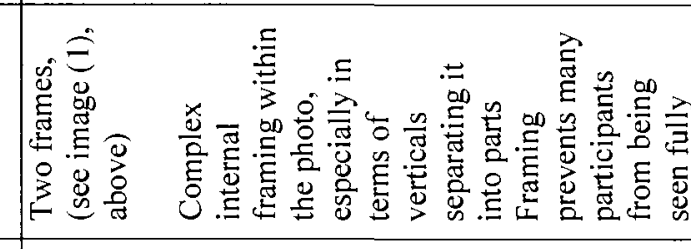 \\
\hline 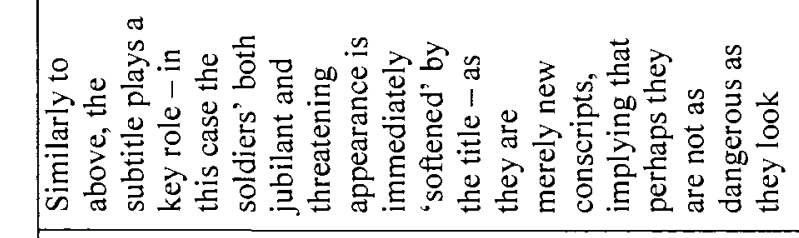 & 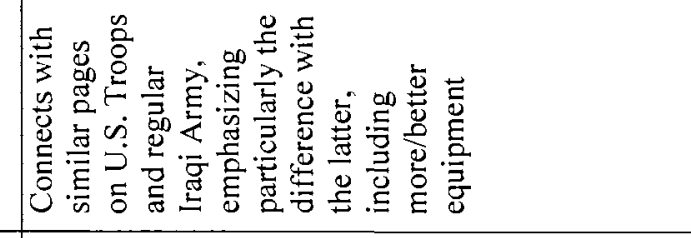 \\
\hline 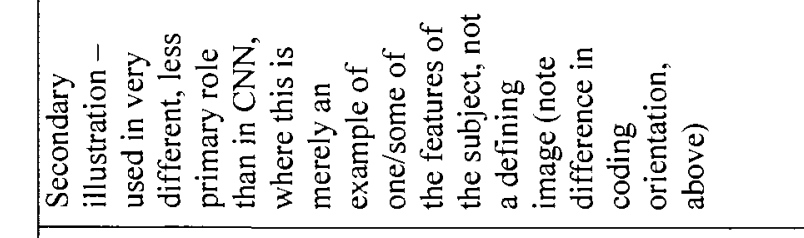 & 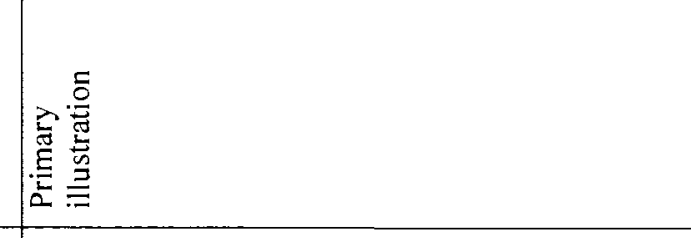 \\
\hline 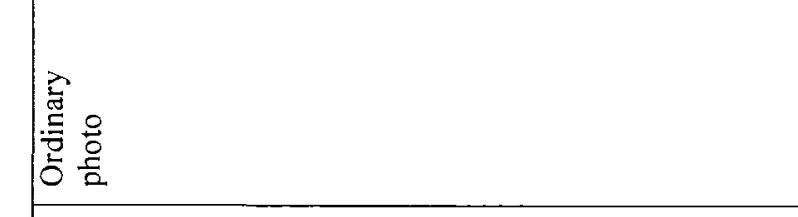 & 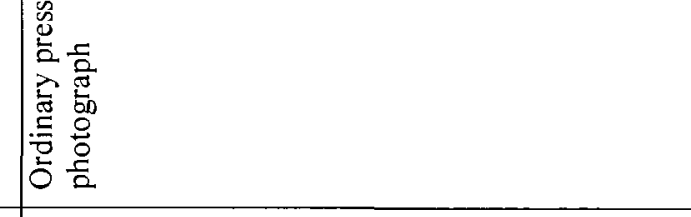 \\
\hline 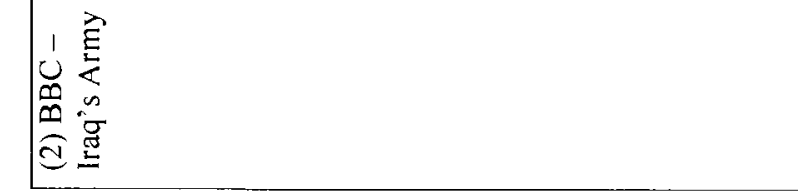 & 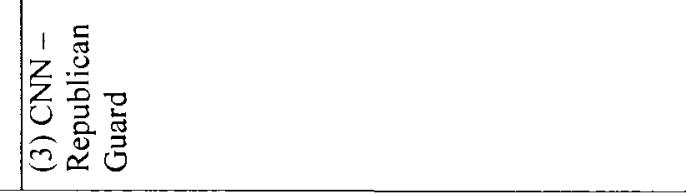 \\
\hline
\end{tabular}




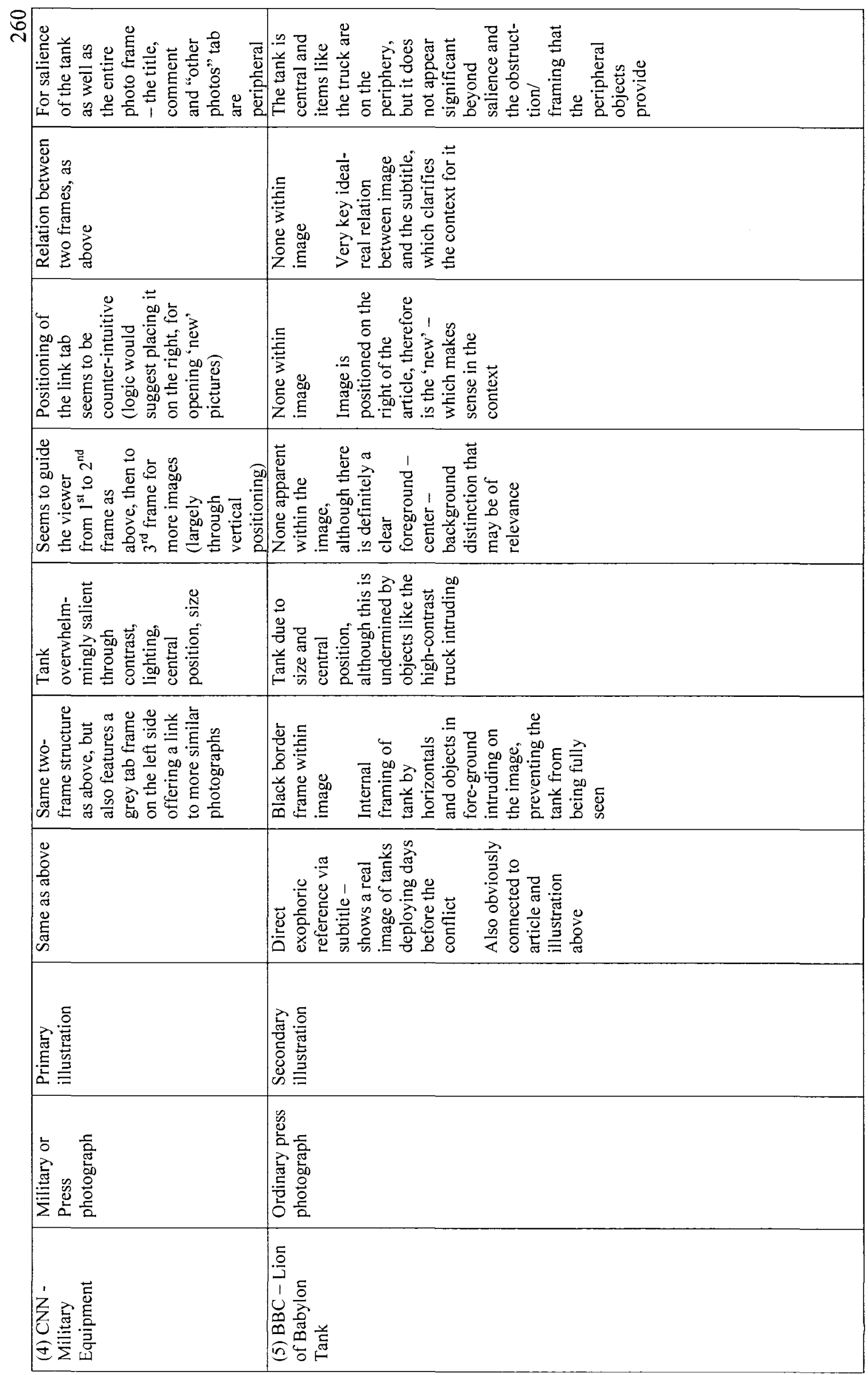




\begin{tabular}{|c|c|}
\hline 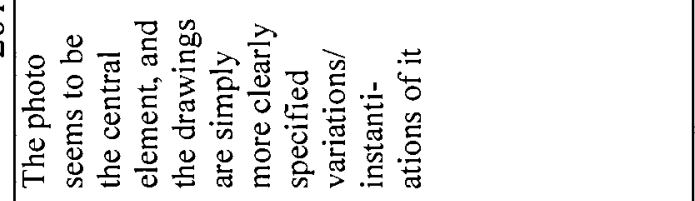 & 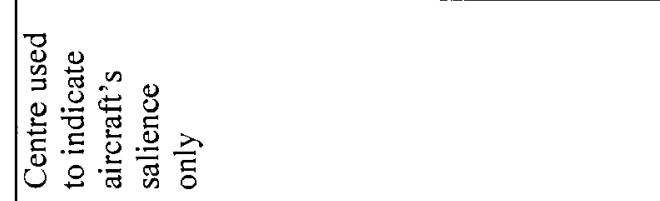 \\
\hline 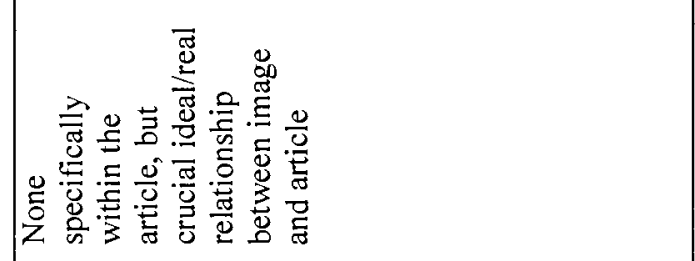 & 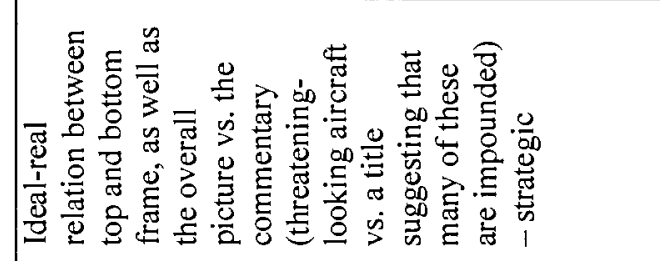 \\
\hline 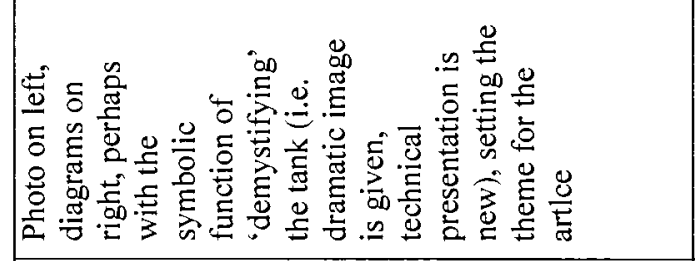 & 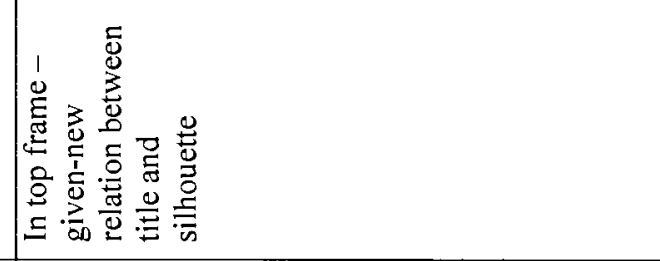 \\
\hline 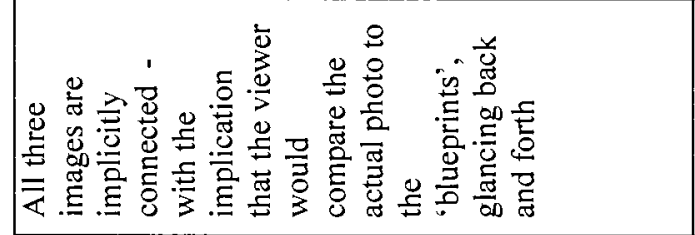 & 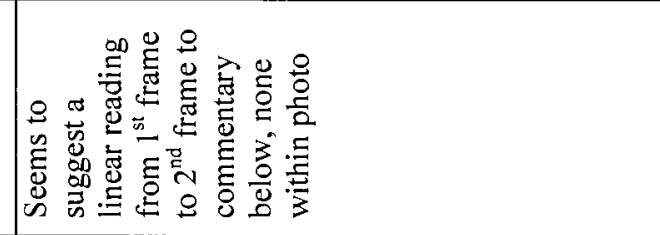 \\
\hline 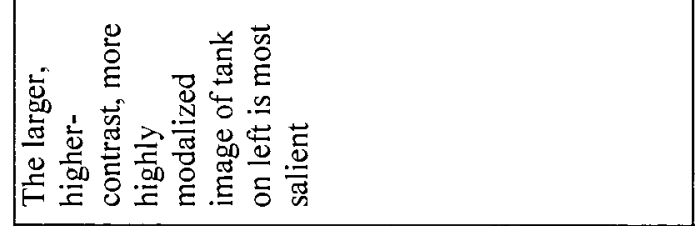 & 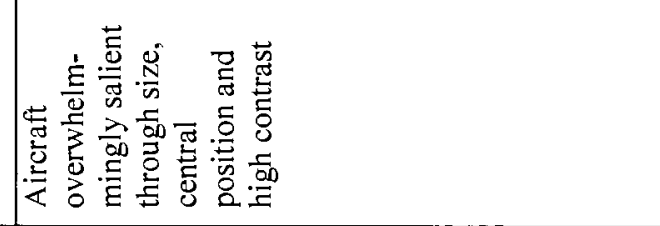 \\
\hline 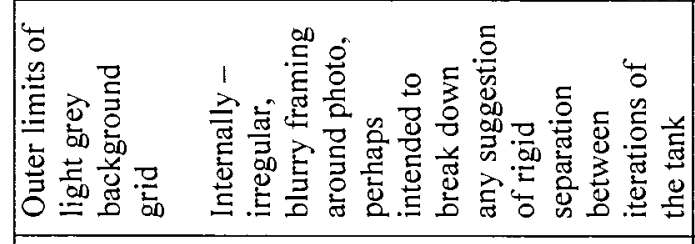 & 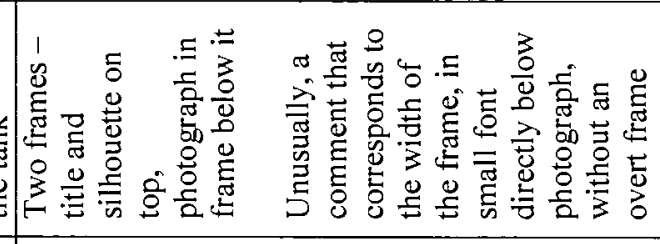 \\
\hline 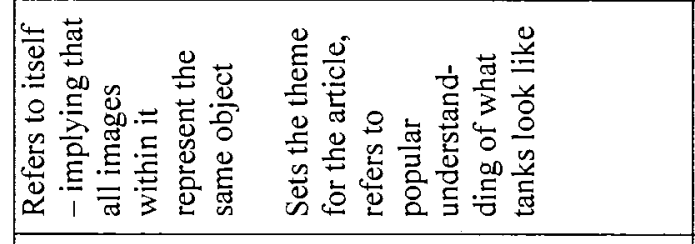 & 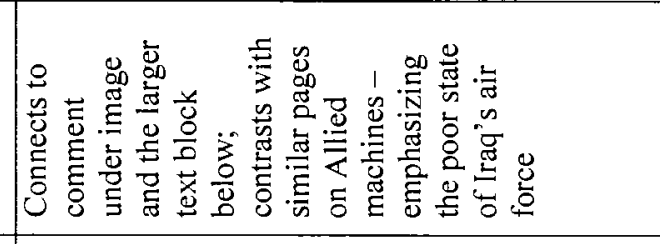 \\
\hline 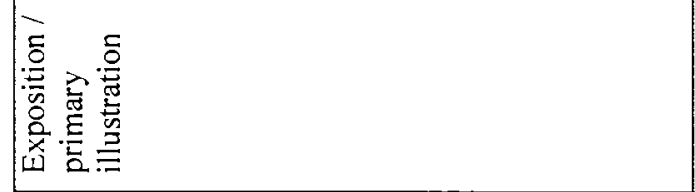 & 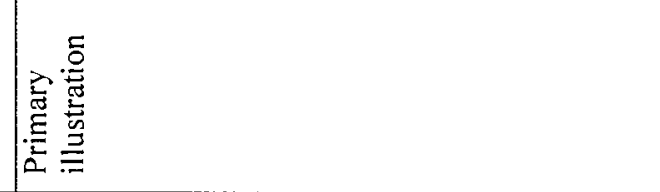 \\
\hline 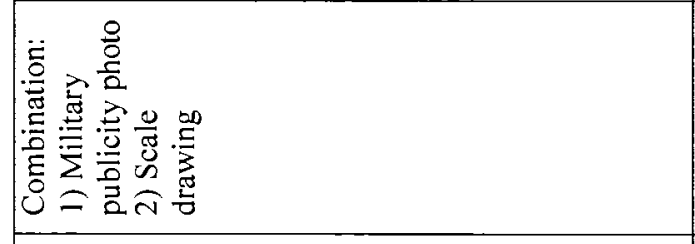 & 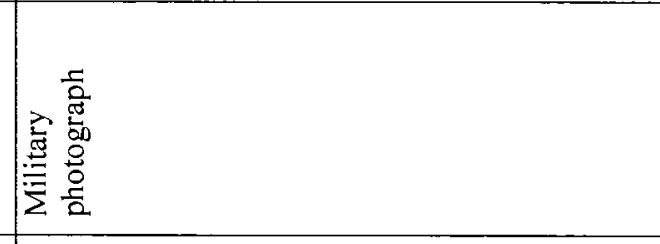 \\
\hline 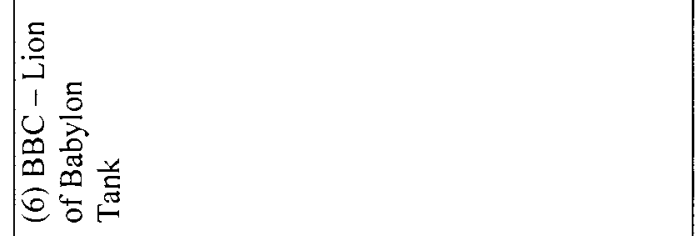 & 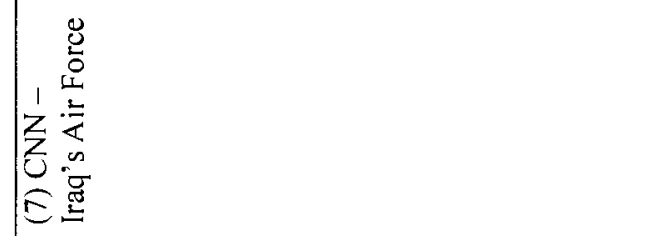 \\
\hline
\end{tabular}




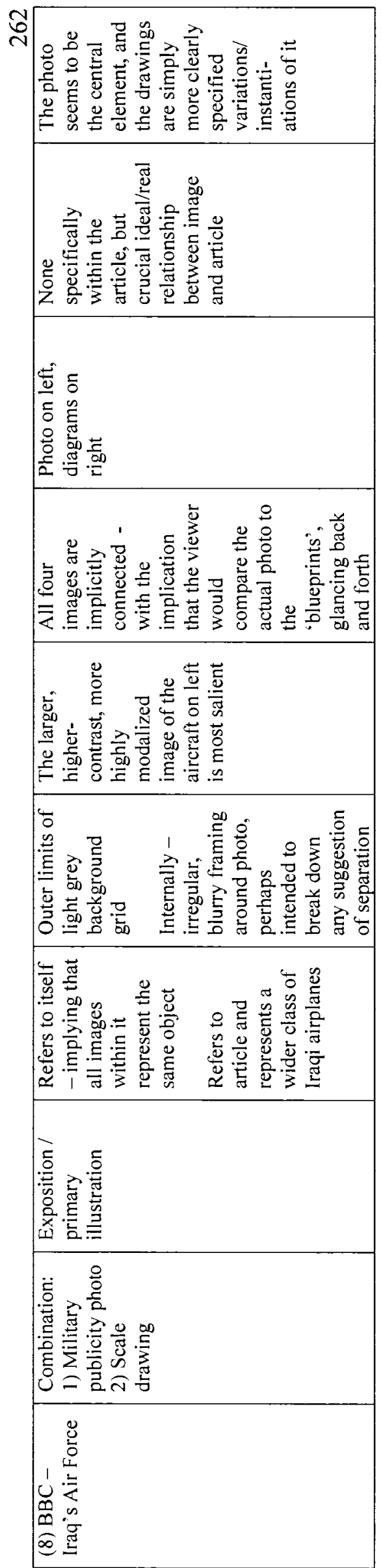




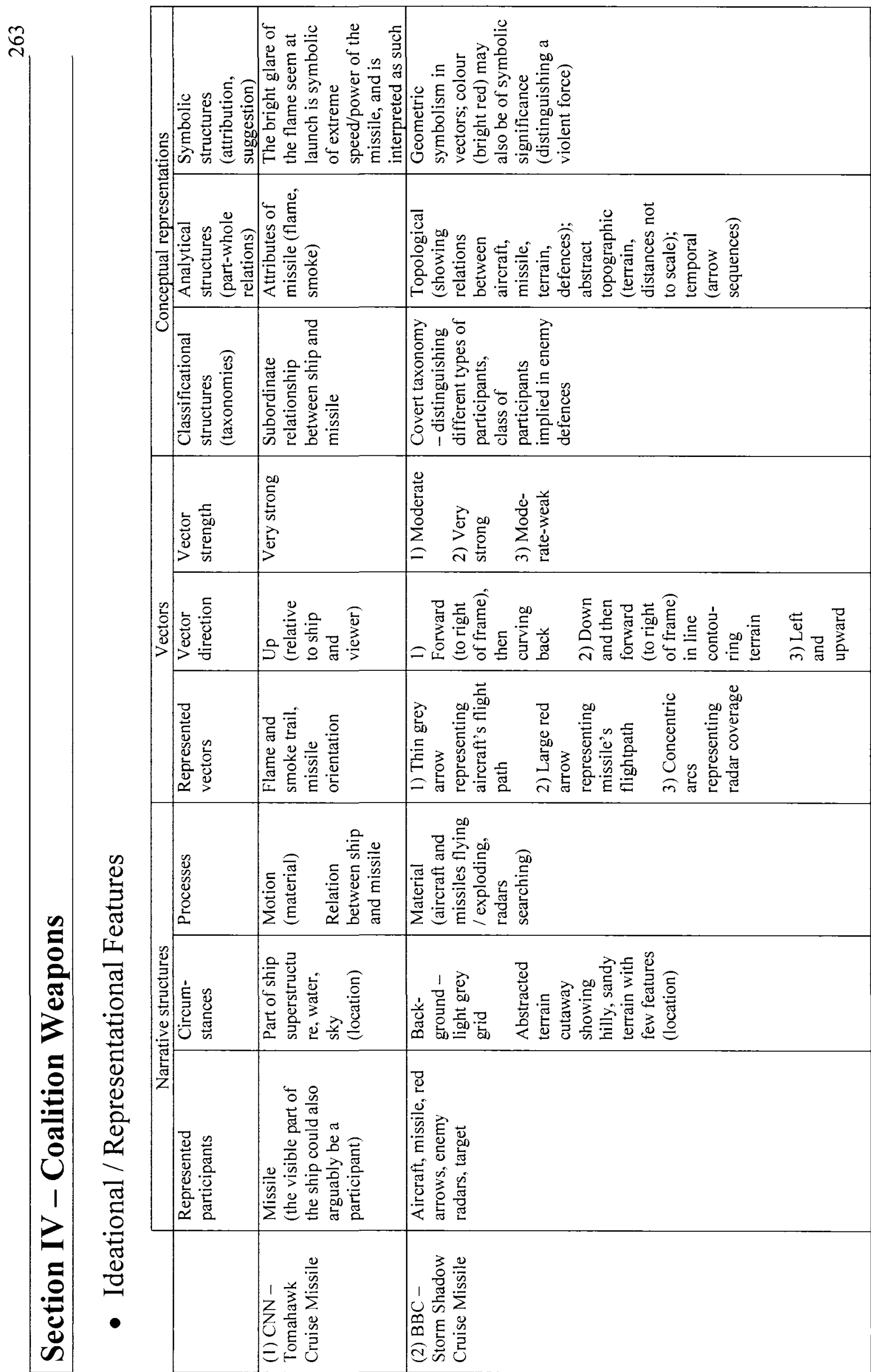




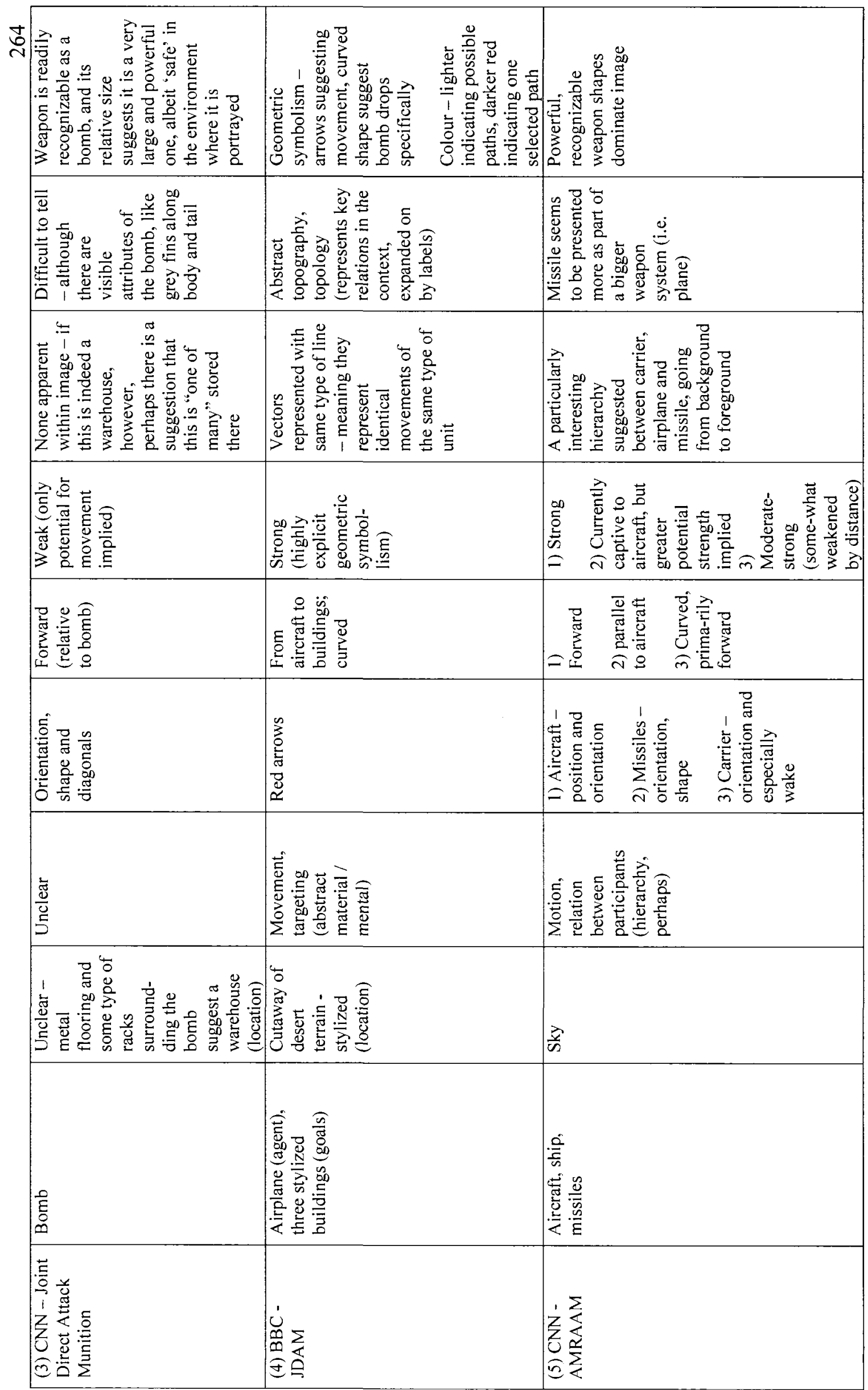




\begin{tabular}{|c|c|c|}
\hline 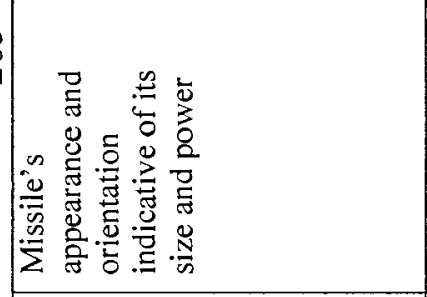 & 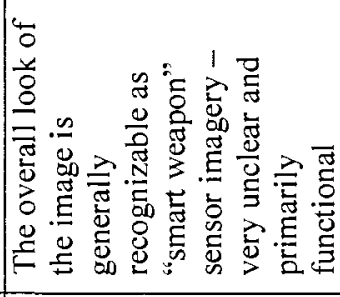 & 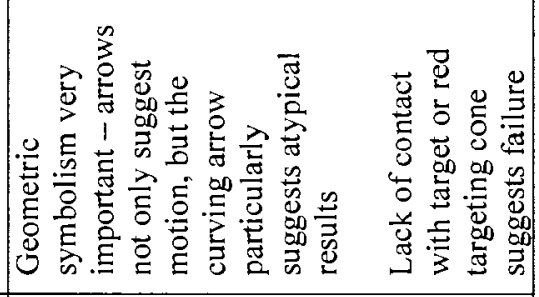 \\
\hline 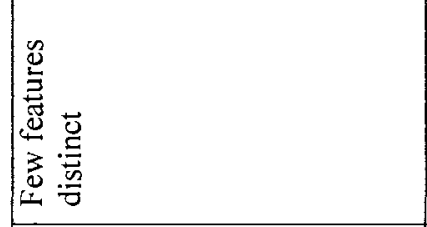 & 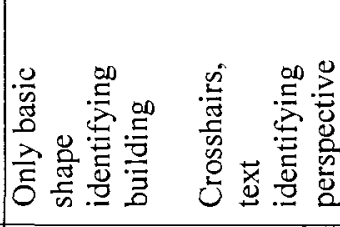 & 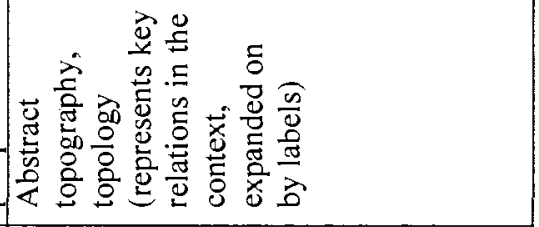 \\
\hline . & . & 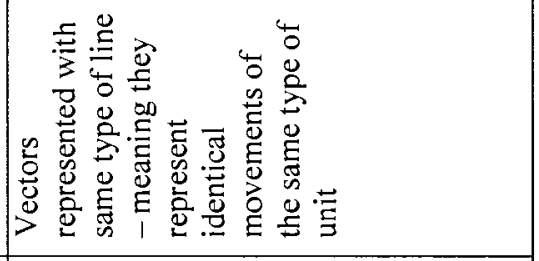 \\
\hline 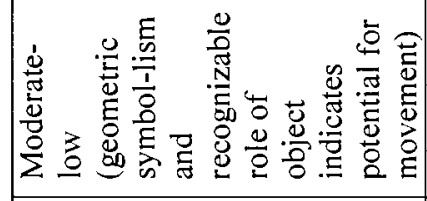 & 然 & 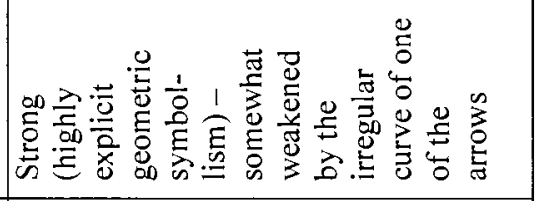 \\
\hline 要 & 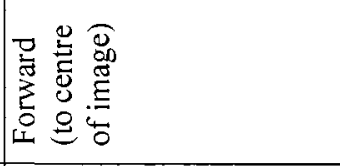 & 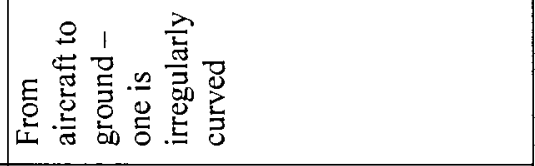 \\
\hline 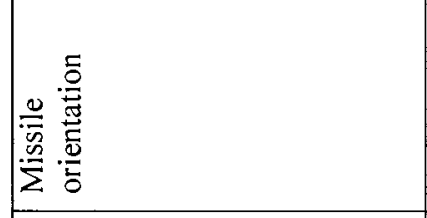 & 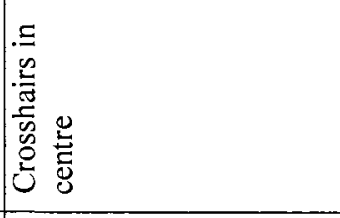 & 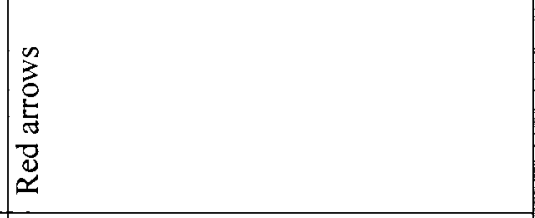 \\
\hline $\begin{array}{l}0 \\
\tilde{L} \\
z \\
z\end{array}$ & 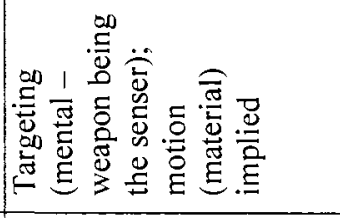 & 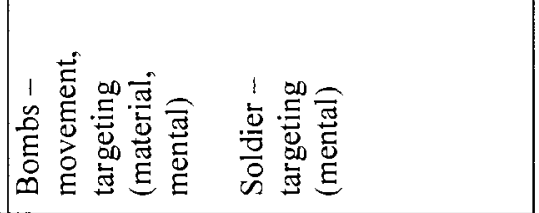 \\
\hline 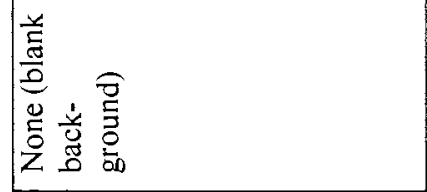 & $\mid \begin{array}{l}\frac{5}{5} \\
\frac{\mathbb{E}}{5} \\
5\end{array}$ & 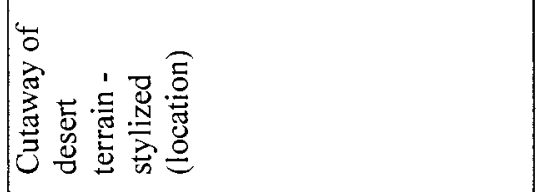 \\
\hline $\begin{array}{l}\frac{0}{5} \\
\bar{z} \\
\Sigma\end{array}$ & 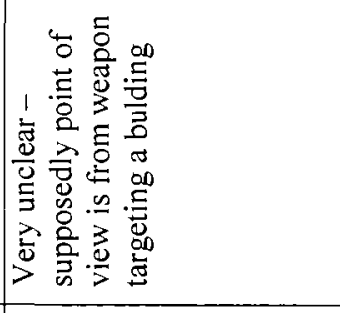 & 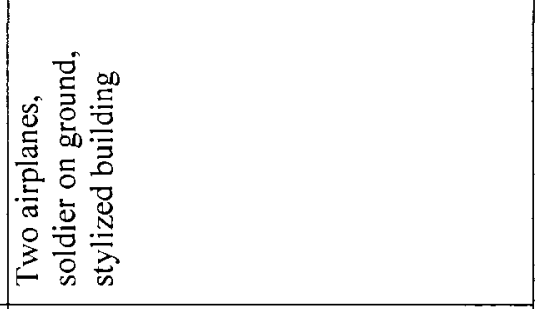 \\
\hline 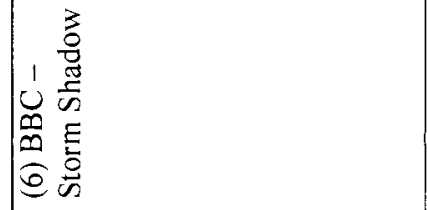 & 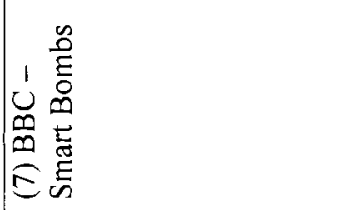 & 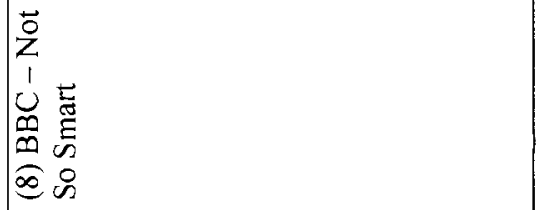 \\
\hline
\end{tabular}




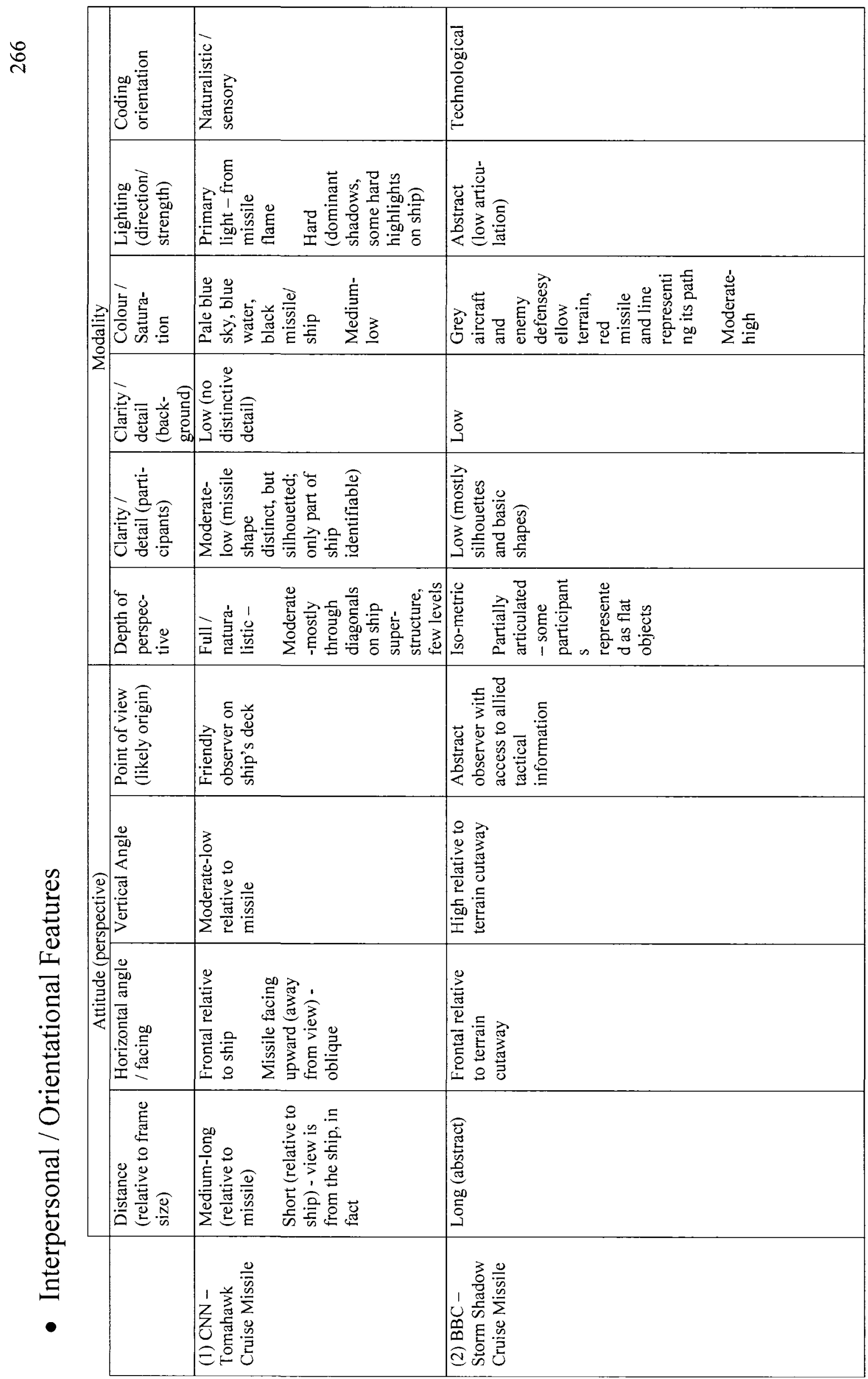




\begin{tabular}{|c|c|}
\hline 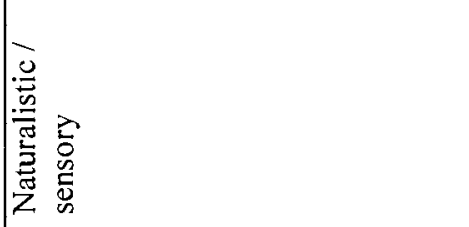 & 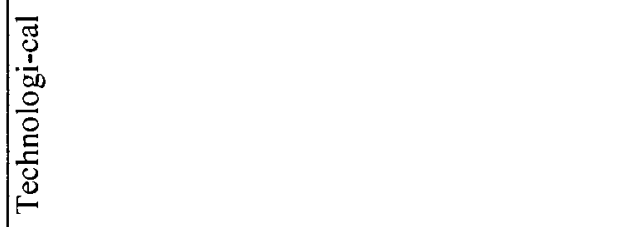 \\
\hline 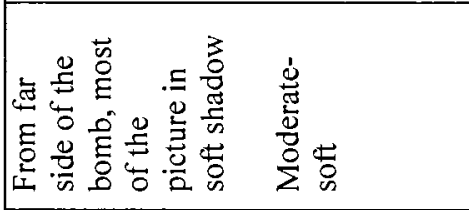 & 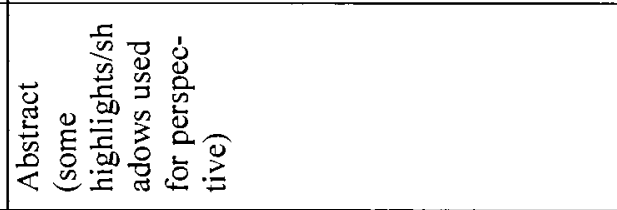 \\
\hline 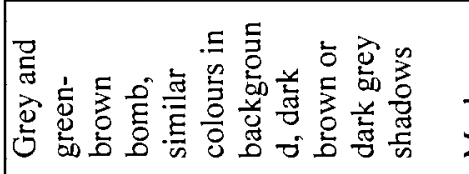 & 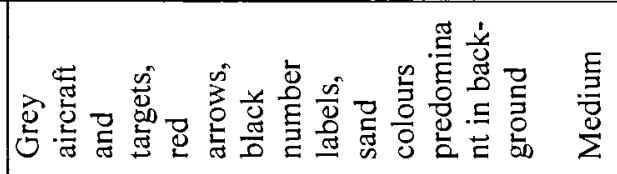 \\
\hline 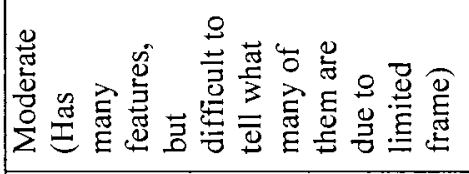 & 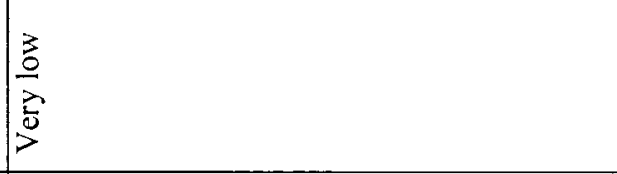 \\
\hline 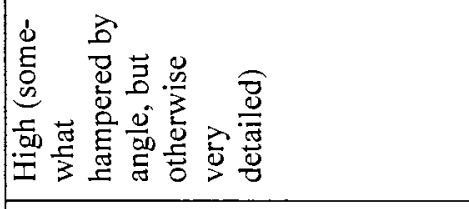 & 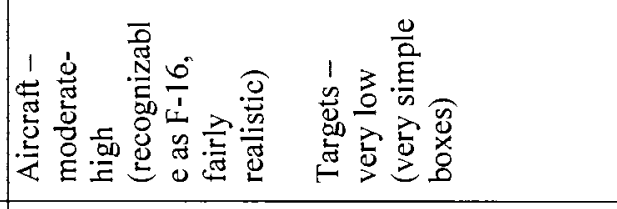 \\
\hline 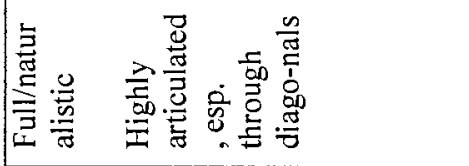 & 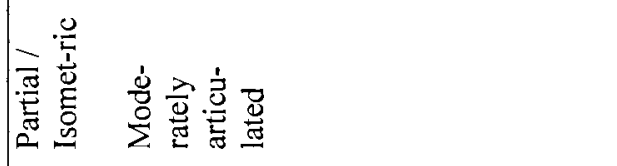 \\
\hline 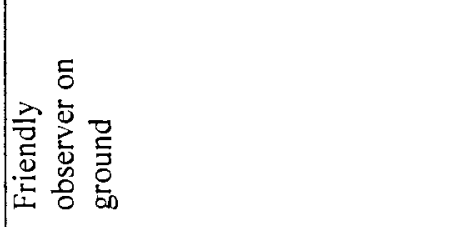 & 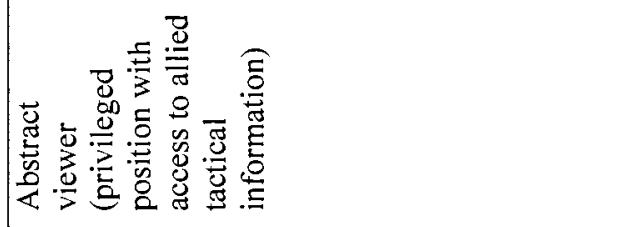 \\
\hline 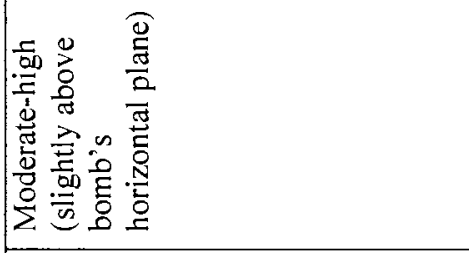 & $\begin{array}{l}-5 \\
\stackrel{5}{ \pm} \\
\end{array}$ \\
\hline 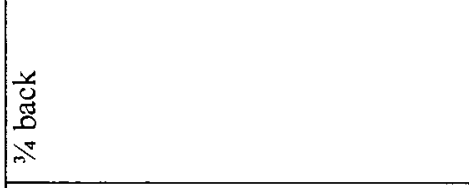 & 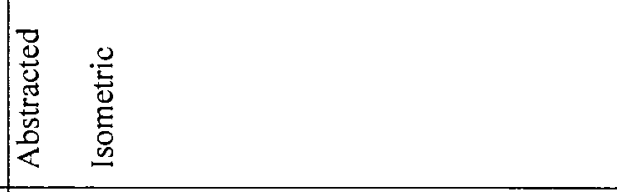 \\
\hline 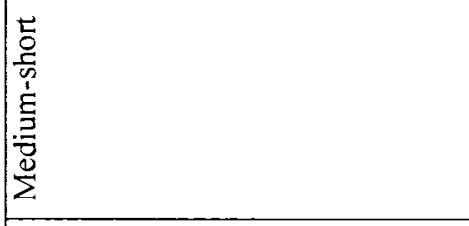 & 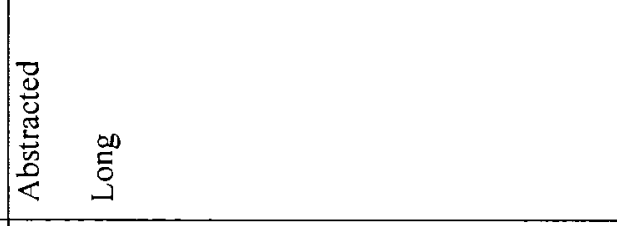 \\
\hline 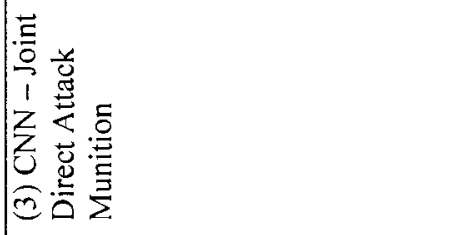 & 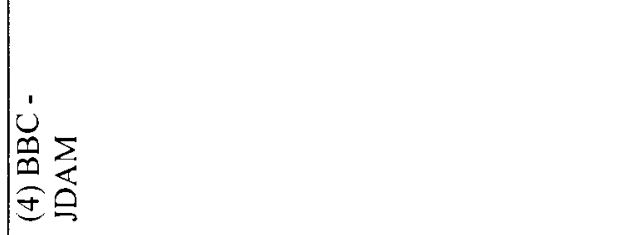 \\
\hline
\end{tabular}




\begin{tabular}{|c|c|}
\hline 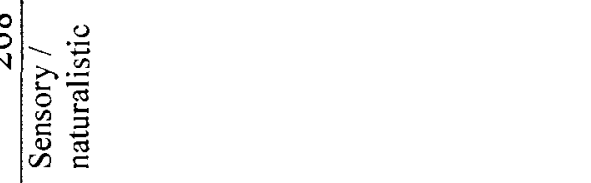 & 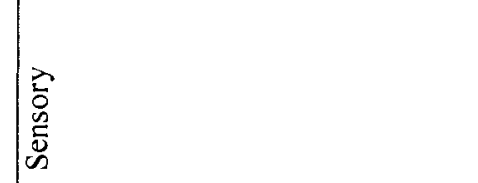 \\
\hline 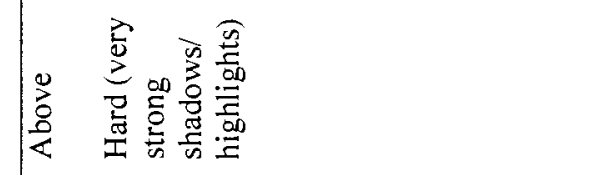 & 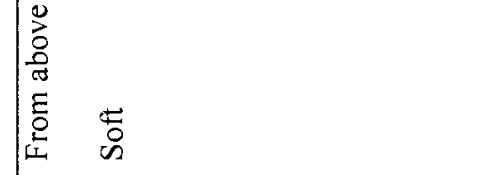 \\
\hline 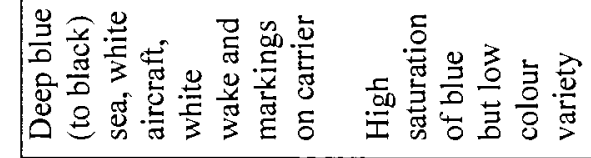 & 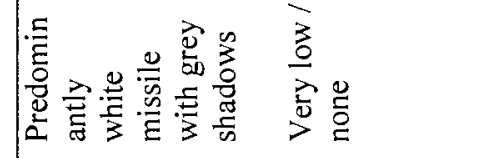 \\
\hline 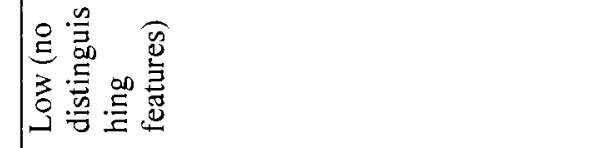 & $\mid \begin{array}{l}\frac{\tilde{g}}{\tilde{z}} \\
\frac{1}{2}\end{array}$ \\
\hline 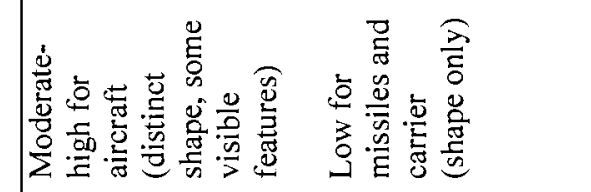 & 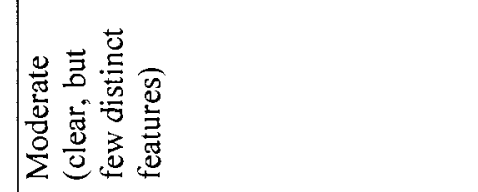 \\
\hline 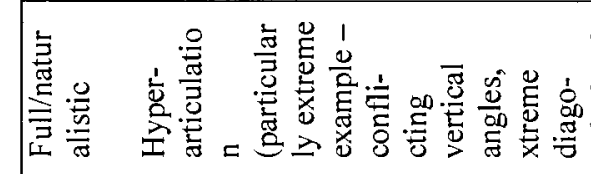 & 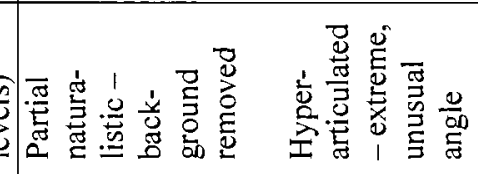 \\
\hline 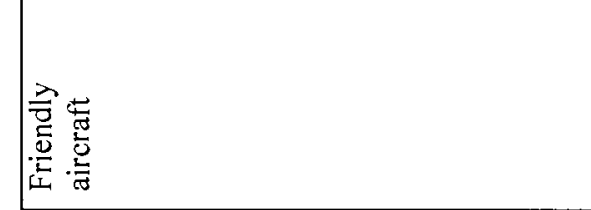 & 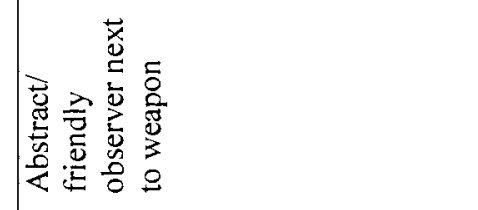 \\
\hline 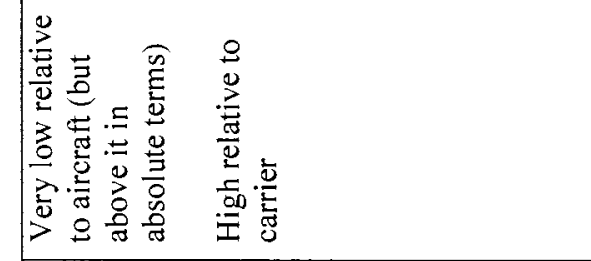 & 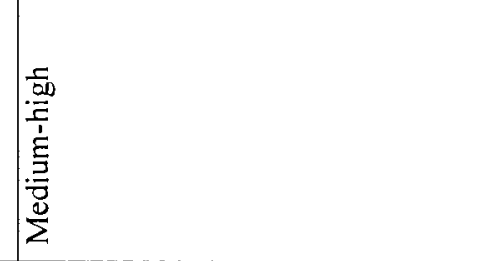 \\
\hline 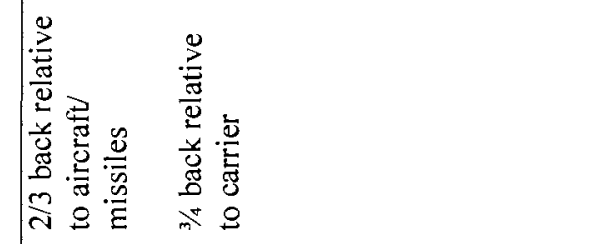 & 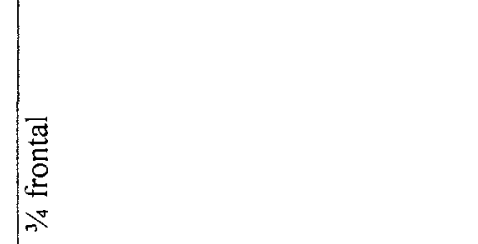 \\
\hline 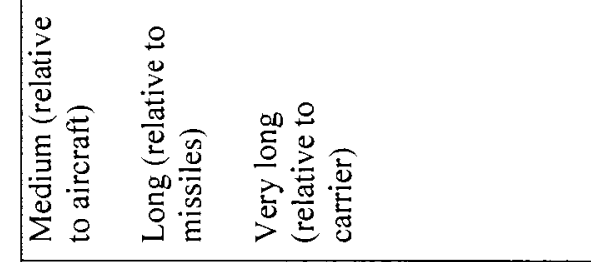 & 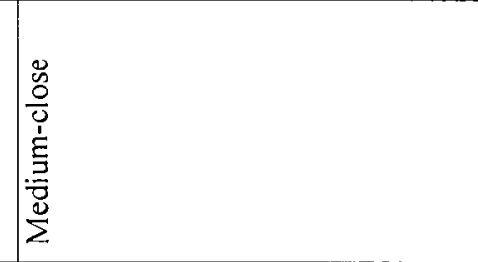 \\
\hline 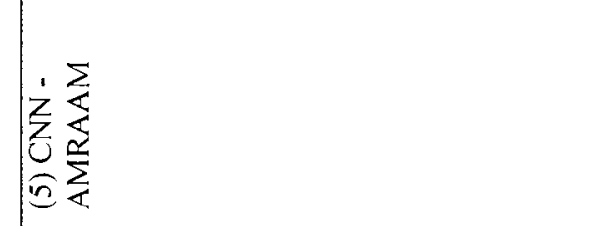 & 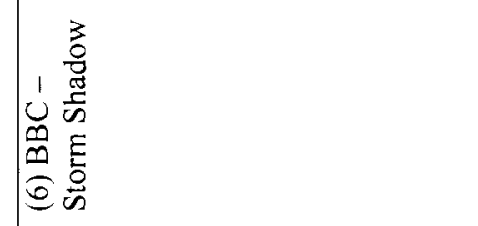 \\
\hline
\end{tabular}




\begin{tabular}{|c|c|}
\hline 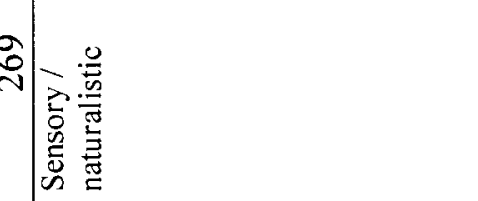 & 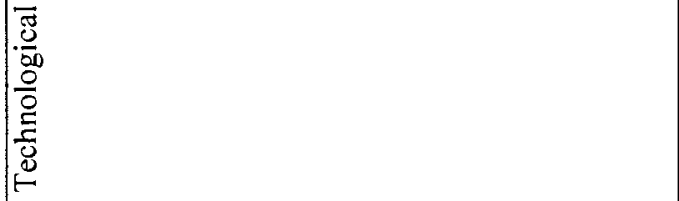 \\
\hline 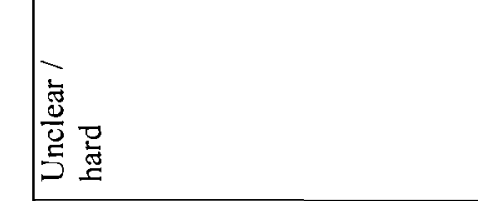 & 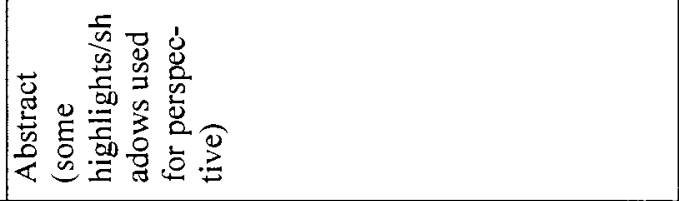 \\
\hline 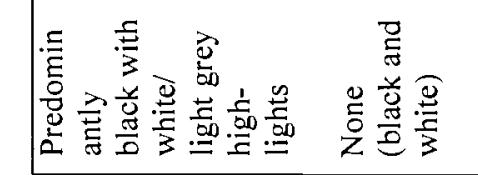 & 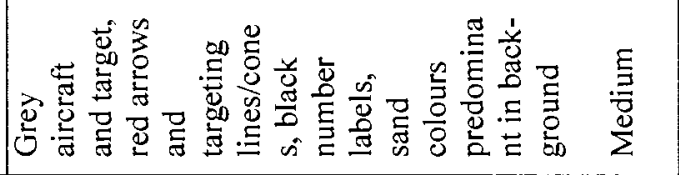 \\
\hline 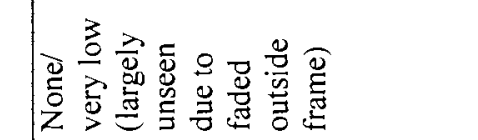 & \\
\hline 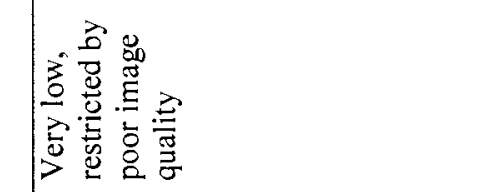 & 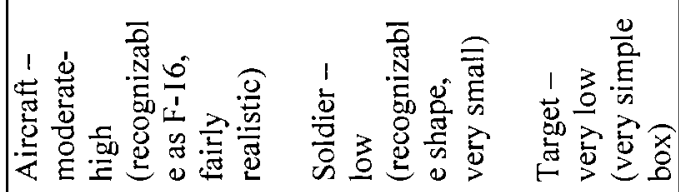 \\
\hline 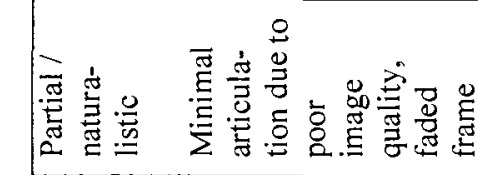 & 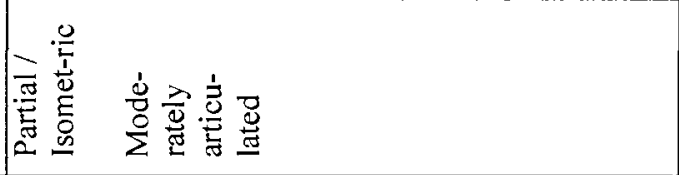 \\
\hline 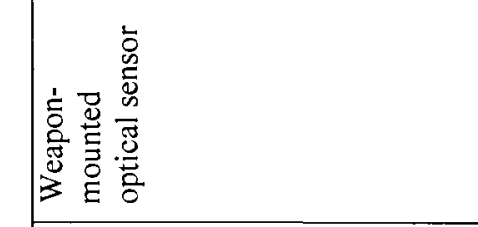 & 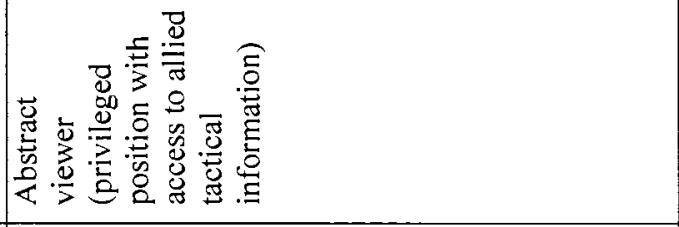 \\
\hline$\frac{-50}{.000}$ & $\frac{c}{\circ}$ \\
\hline 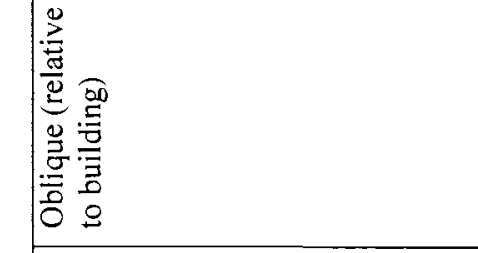 & 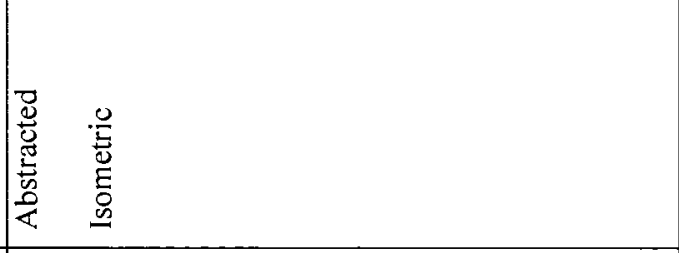 \\
\hline 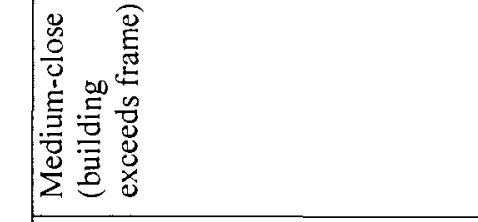 & 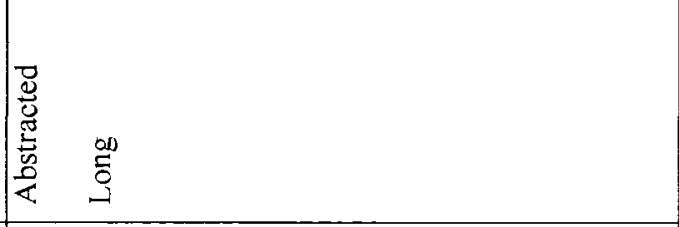 \\
\hline 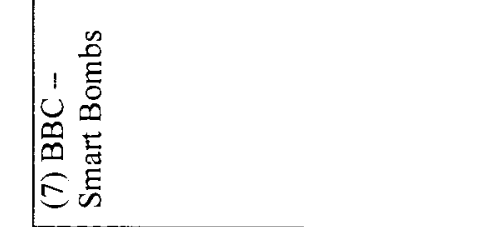 & 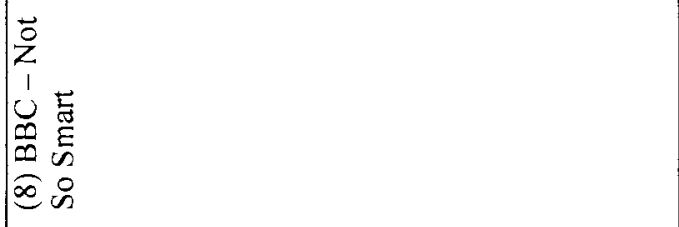 \\
\hline
\end{tabular}


$\stackrel{8}{2}$

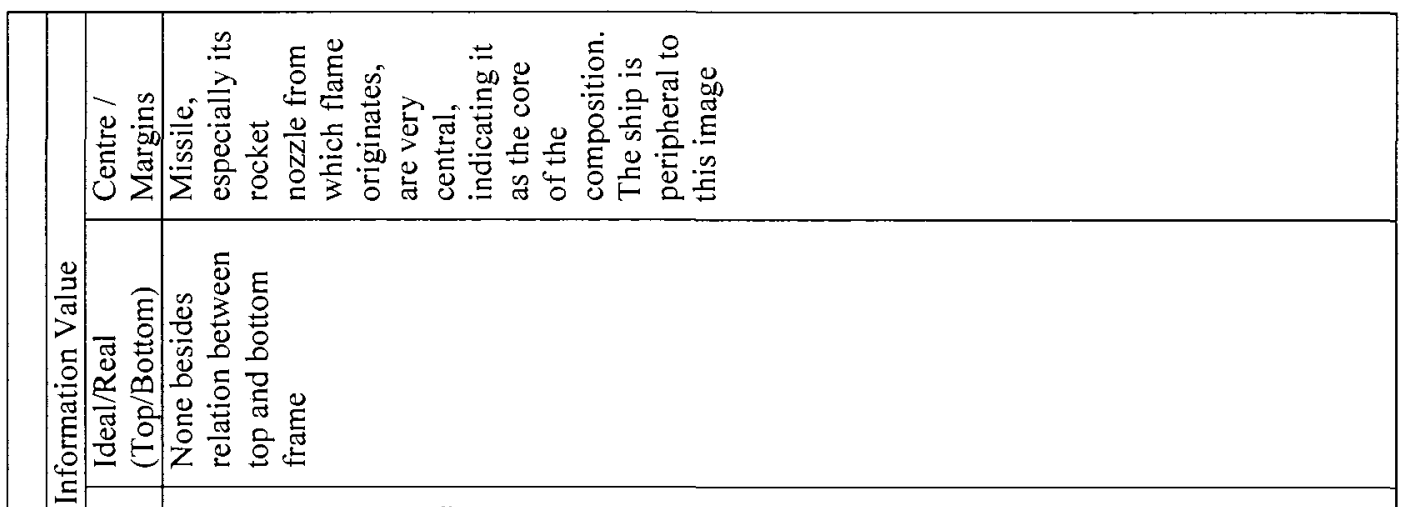

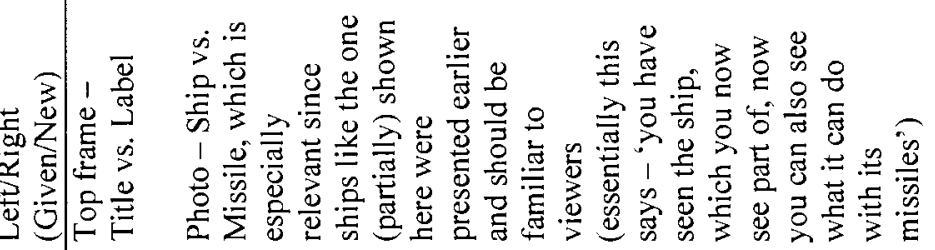

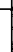

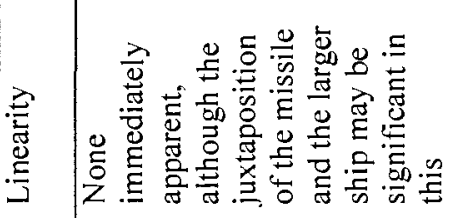

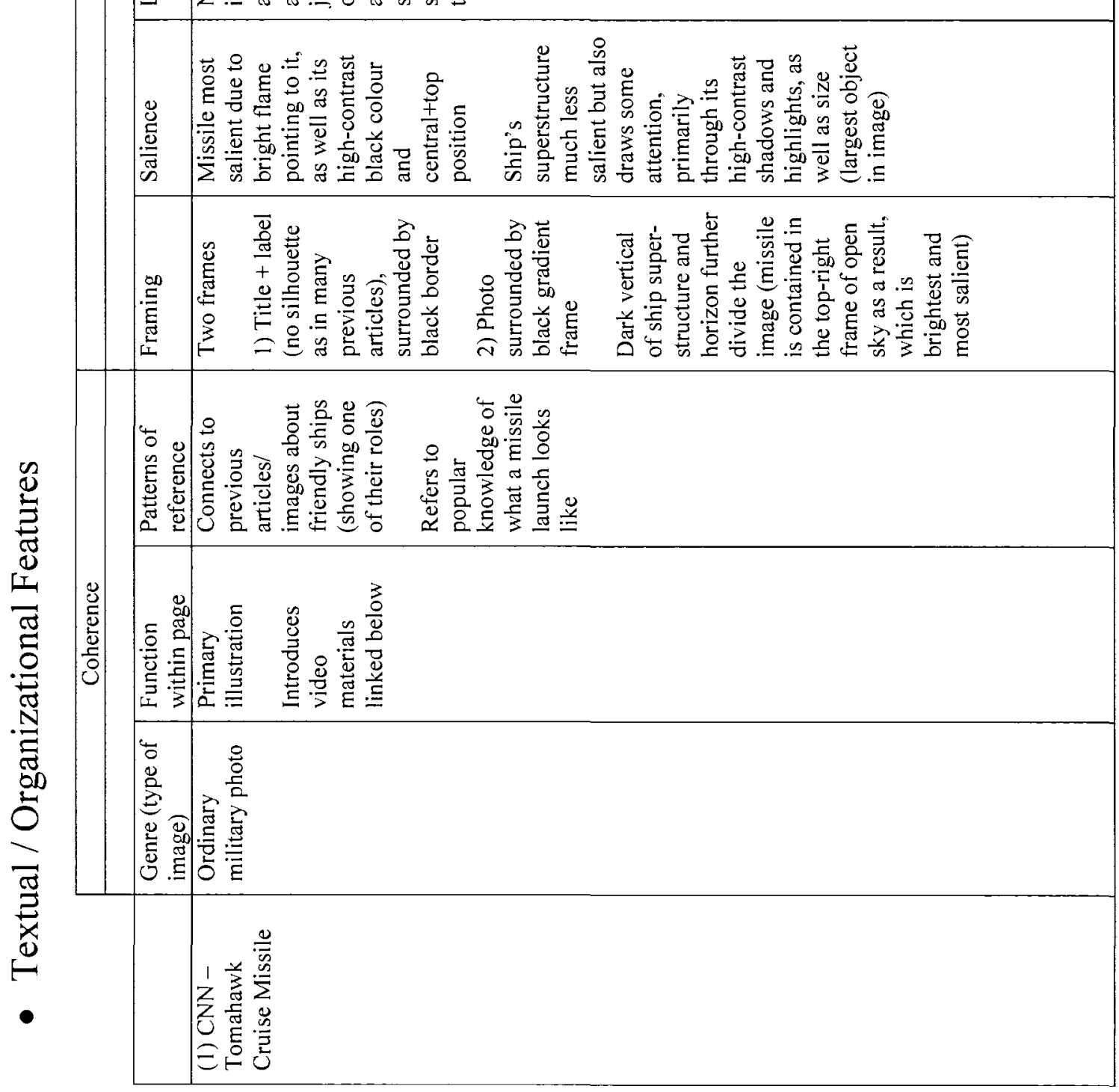




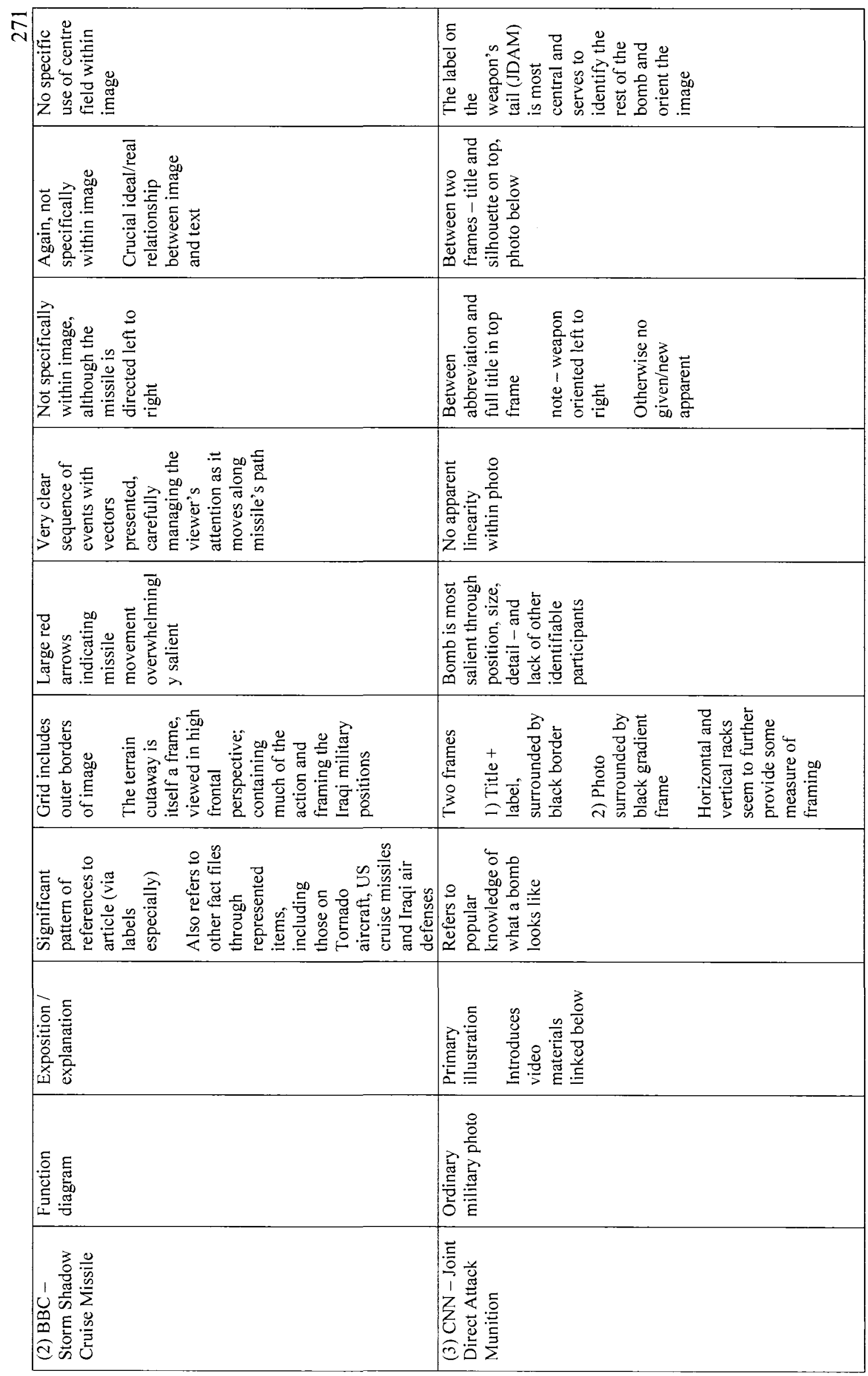




\begin{tabular}{|c|c|}
\hline 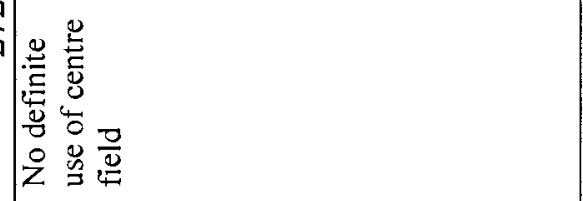 & 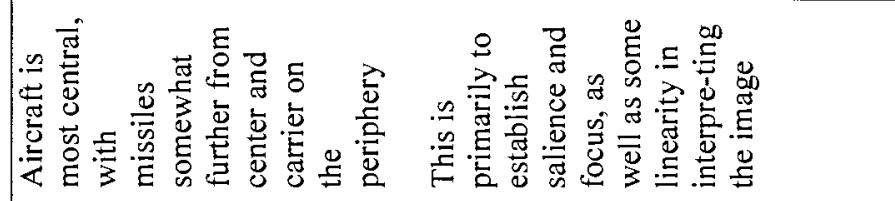 \\
\hline 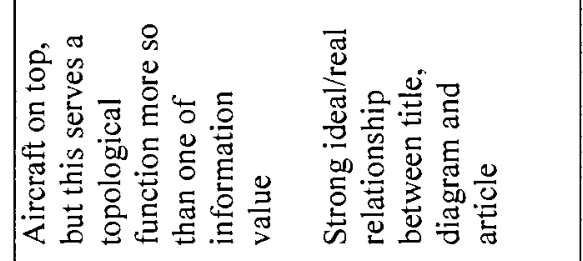 & 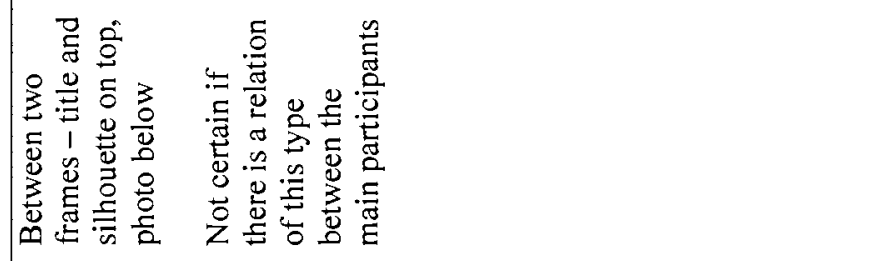 \\
\hline 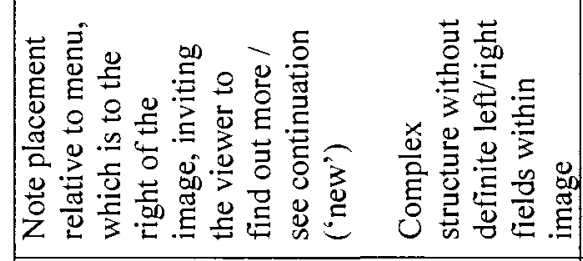 & 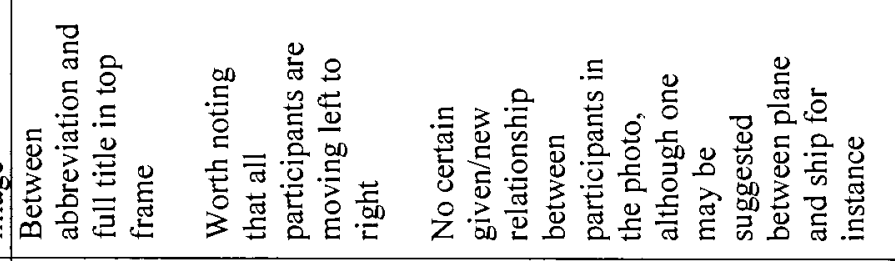 \\
\hline 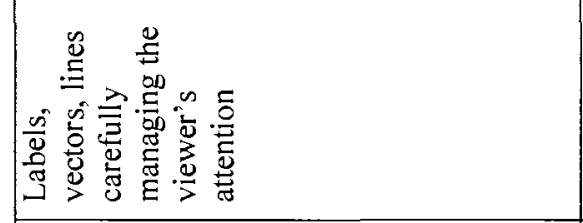 & 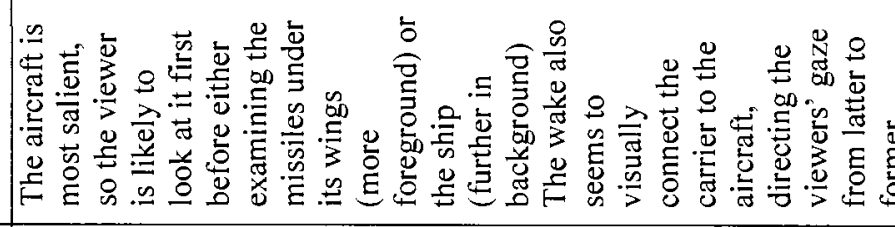 \\
\hline 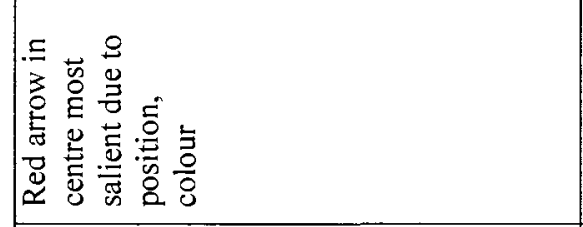 & 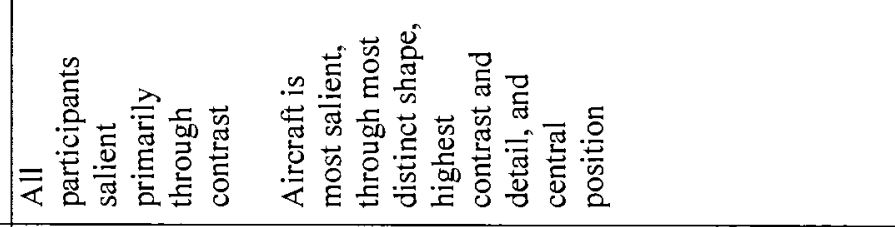 \\
\hline 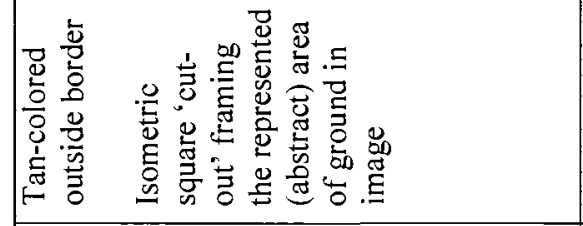 & 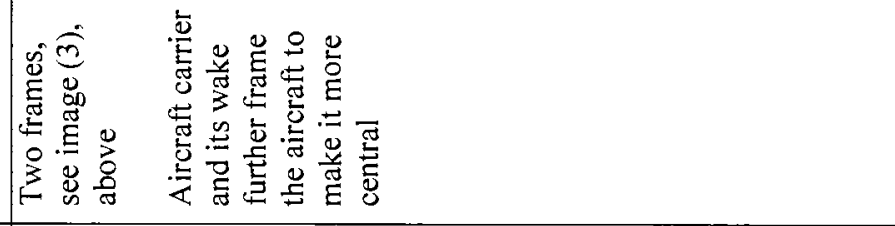 \\
\hline 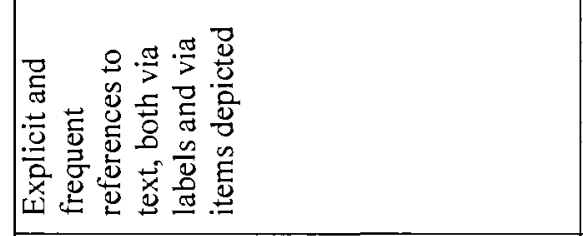 & 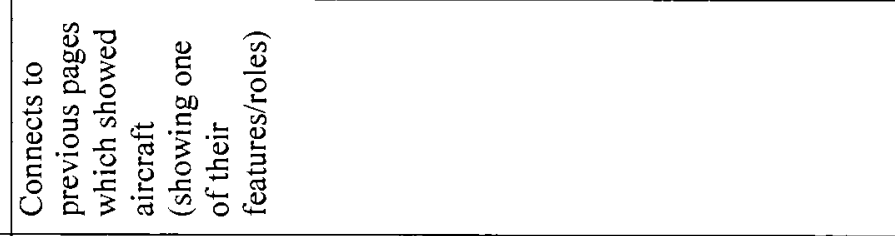 \\
\hline 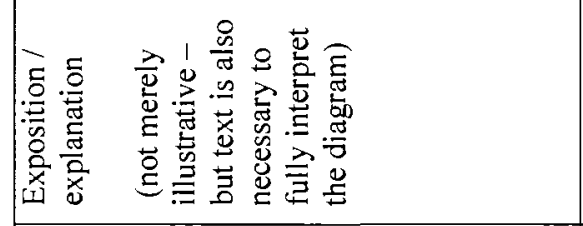 & 竞 \\
\hline 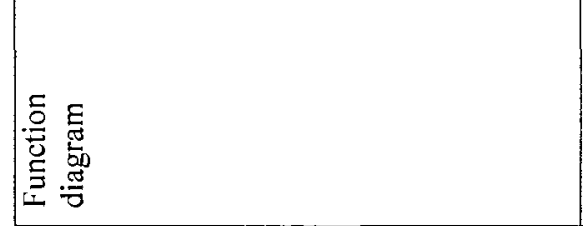 & 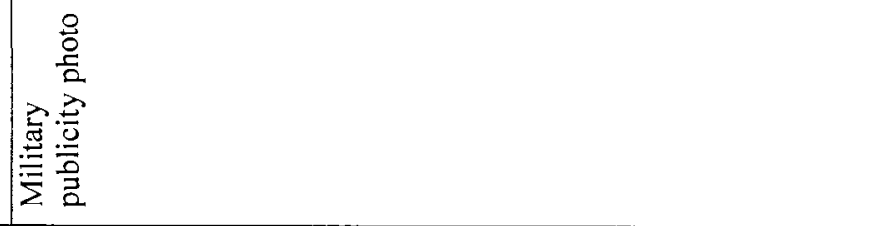 \\
\hline 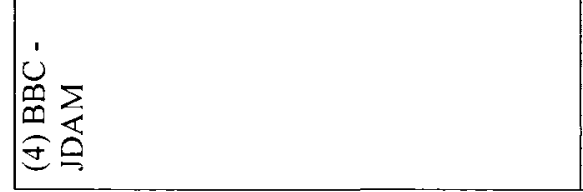 & 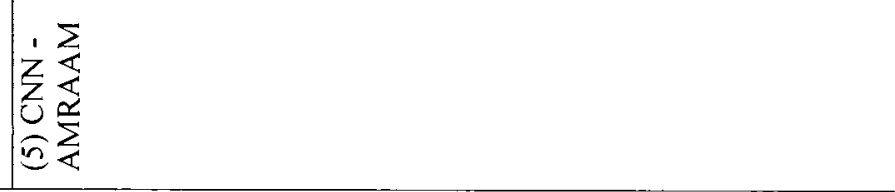 \\
\hline
\end{tabular}




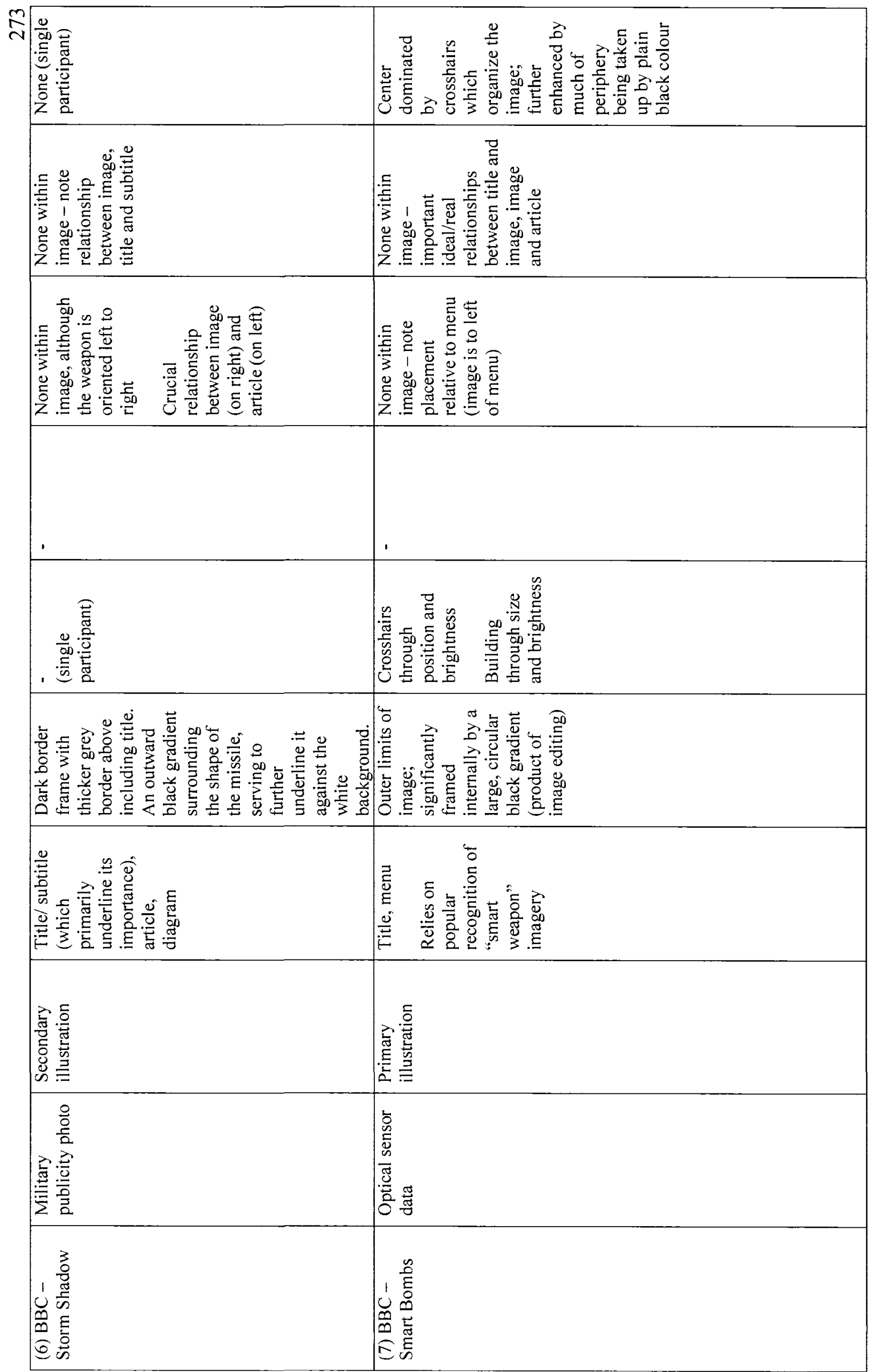




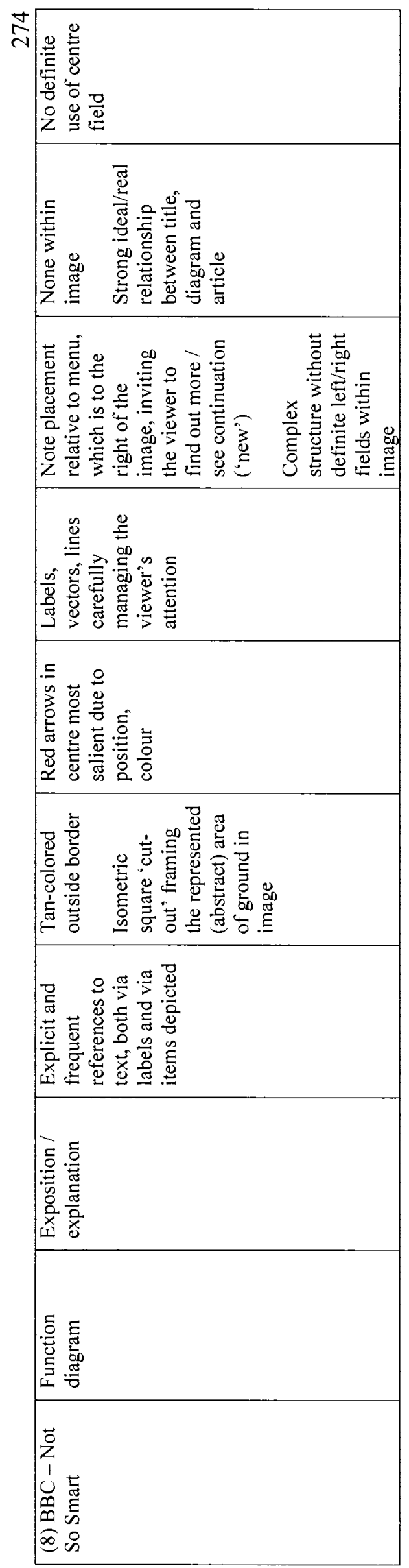


$\stackrel{n}{2}$

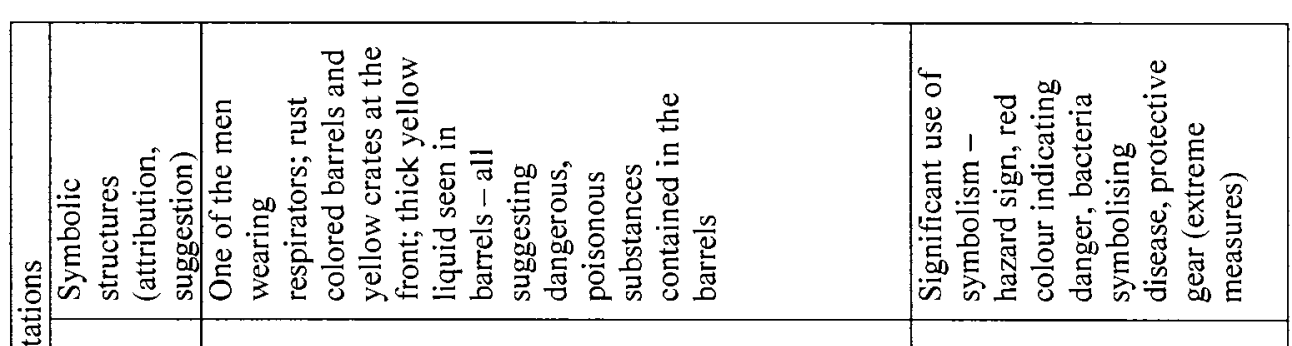

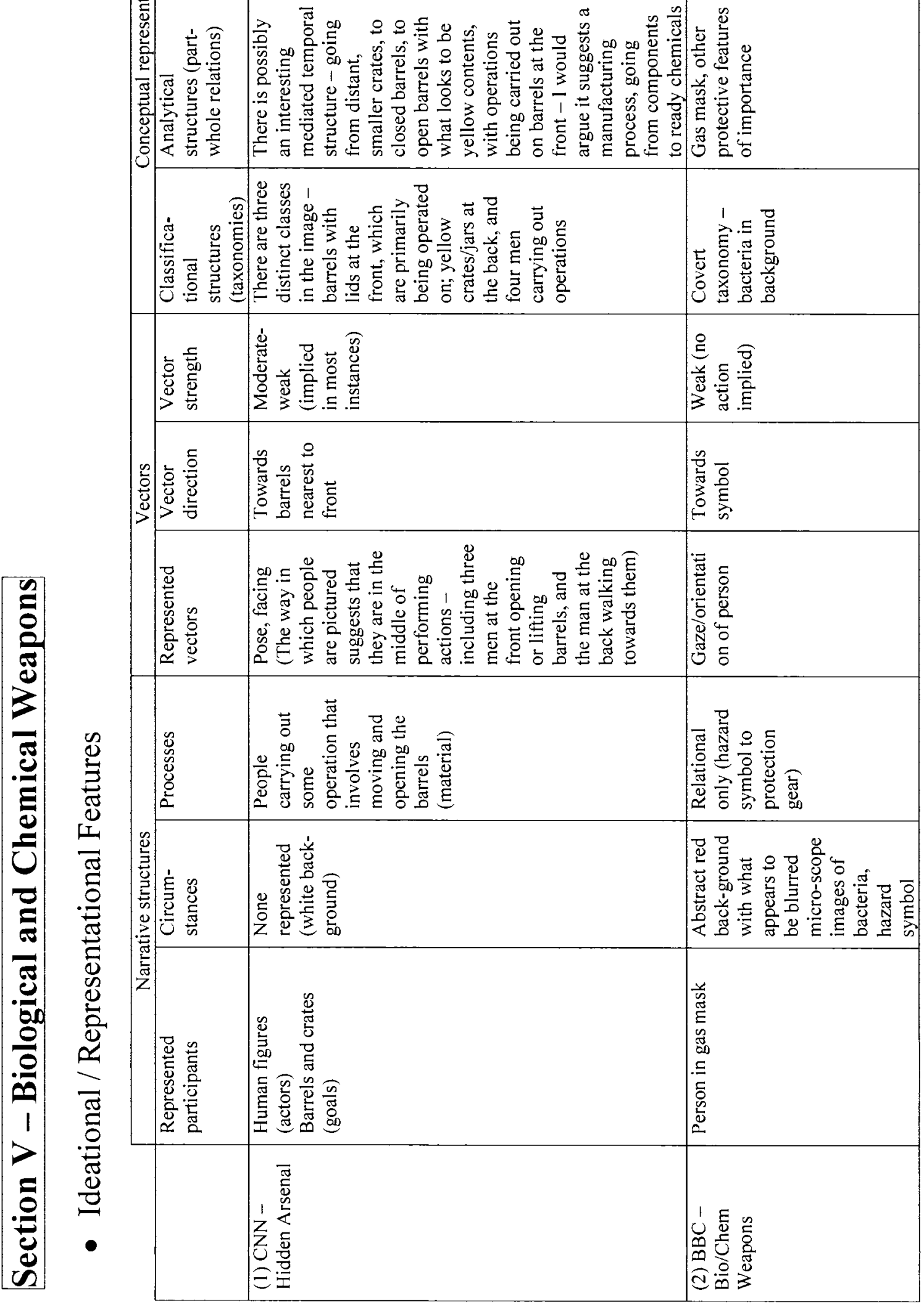




\begin{tabular}{|c|c|}
\hline 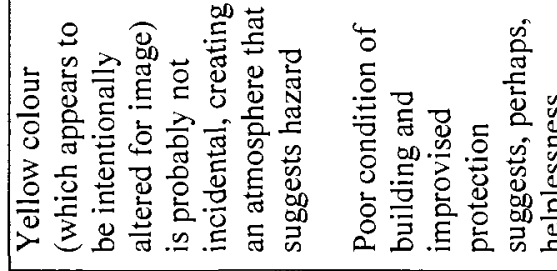 & 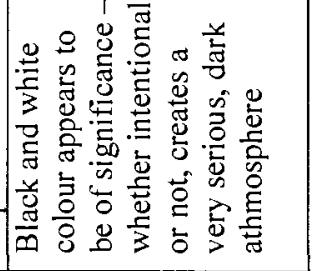 \\
\hline 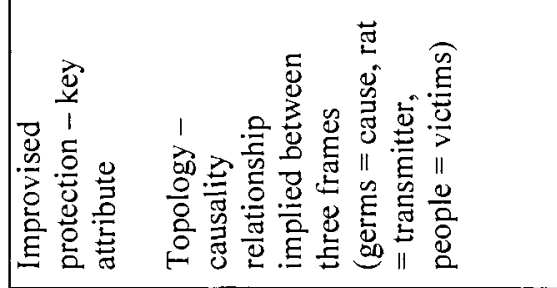 & 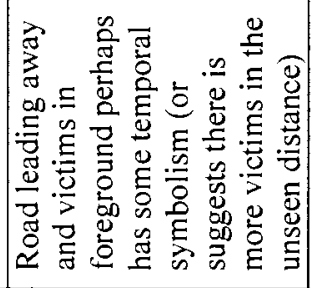 \\
\hline 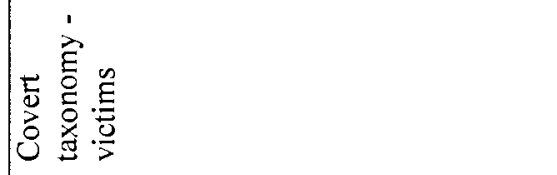 & 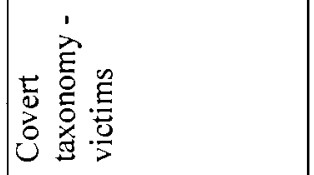 \\
\hline 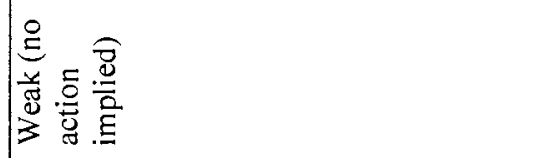 & 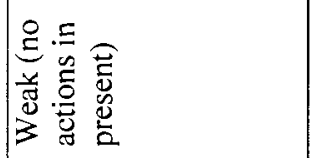 \\
\hline 总 & 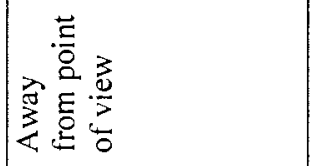 \\
\hline 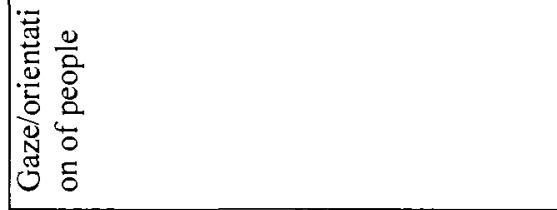 & 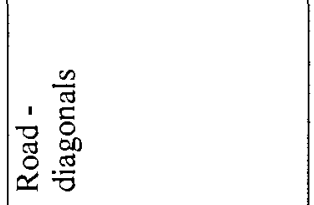 \\
\hline 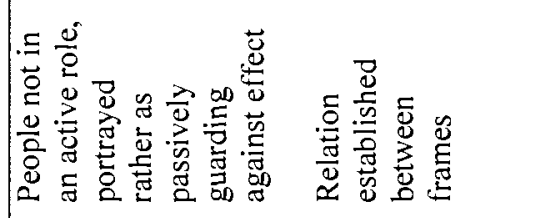 & 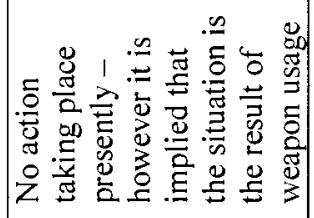 \\
\hline 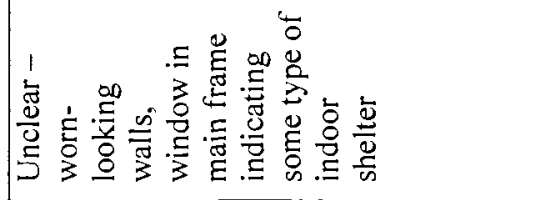 & 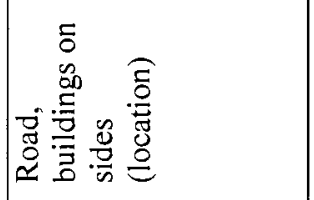 \\
\hline 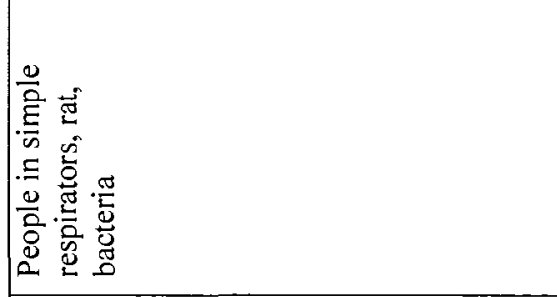 & 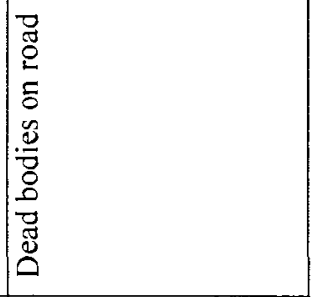 \\
\hline 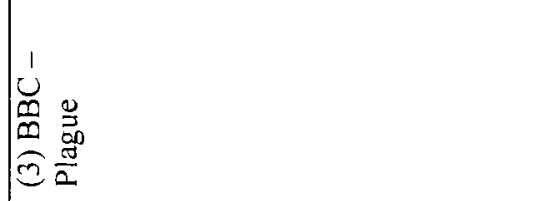 & 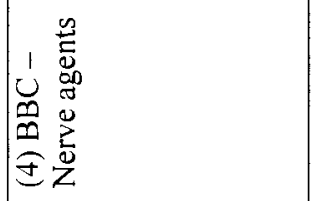 \\
\hline
\end{tabular}




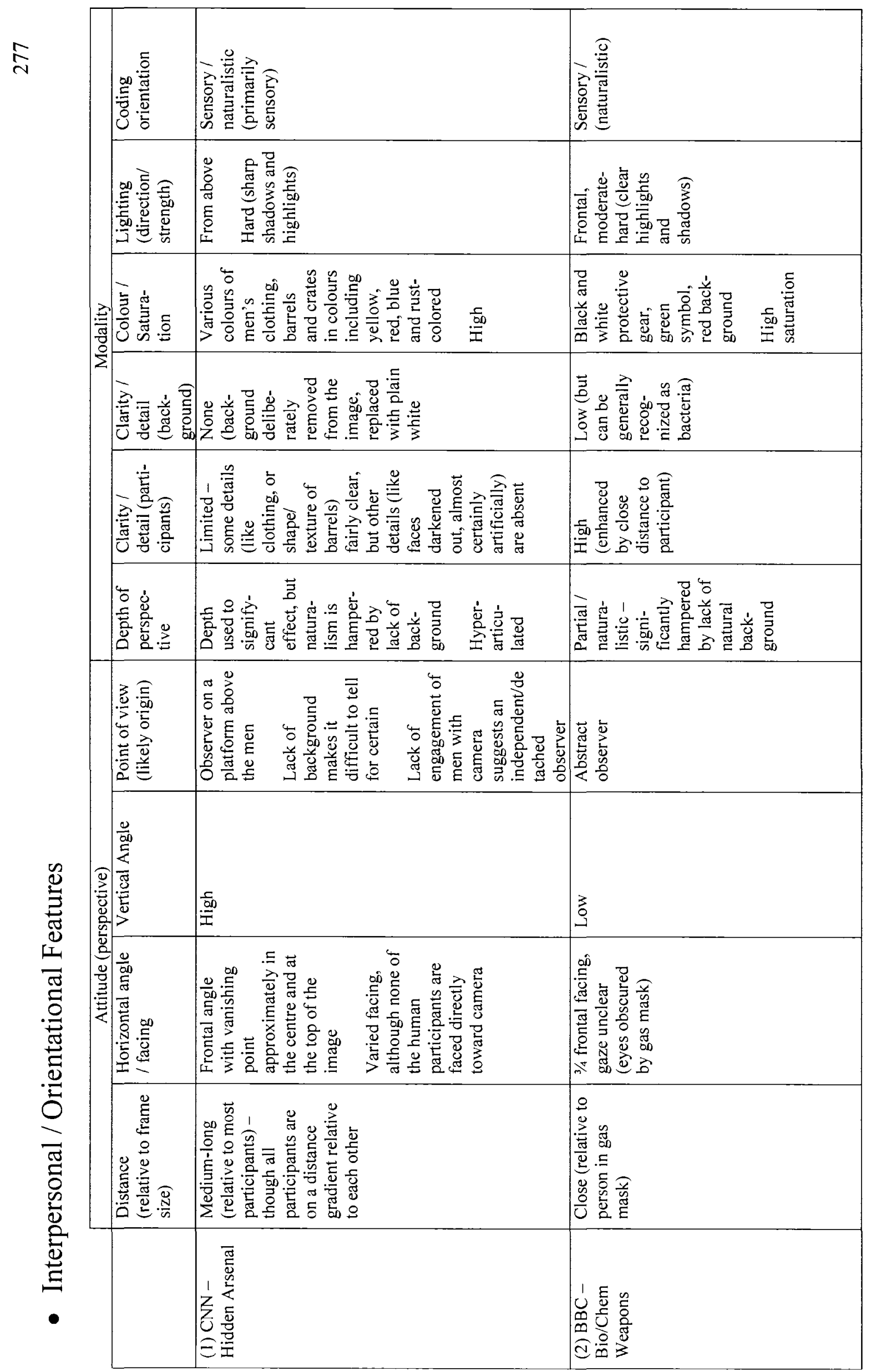




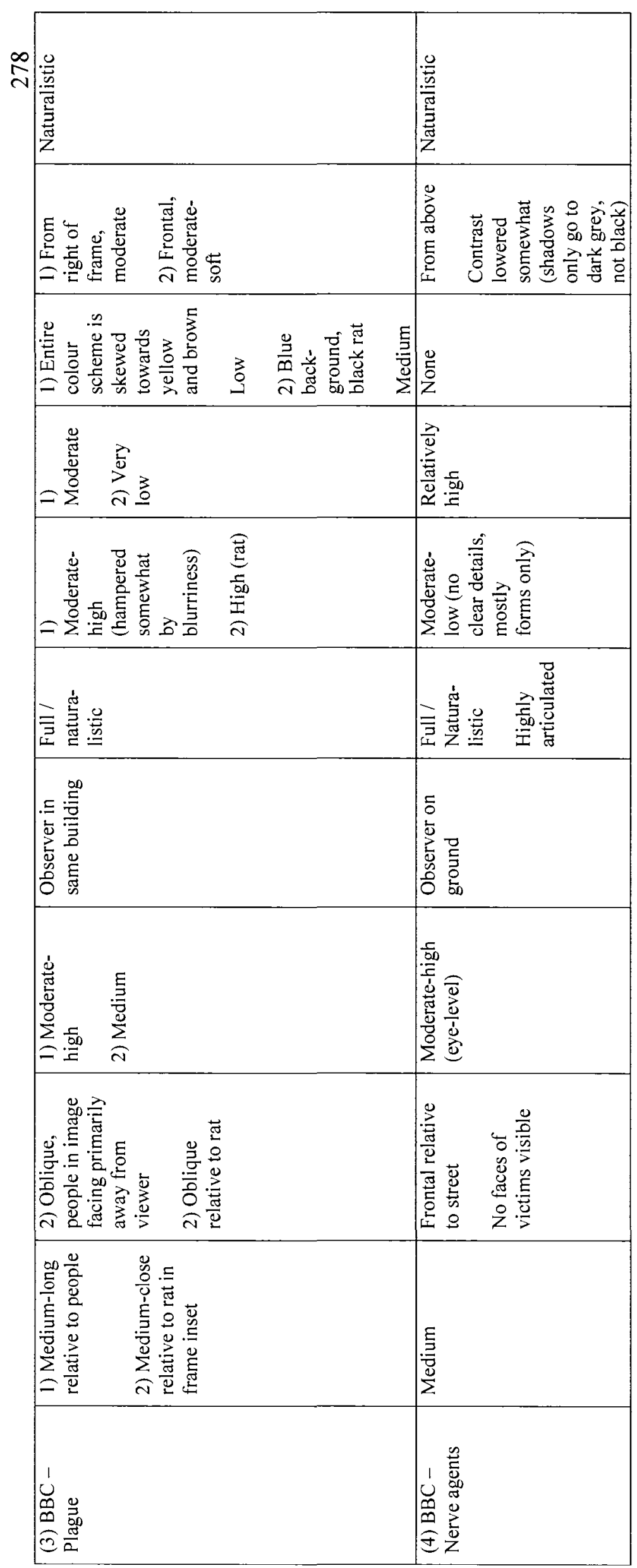


$\stackrel{ }{\sim}$

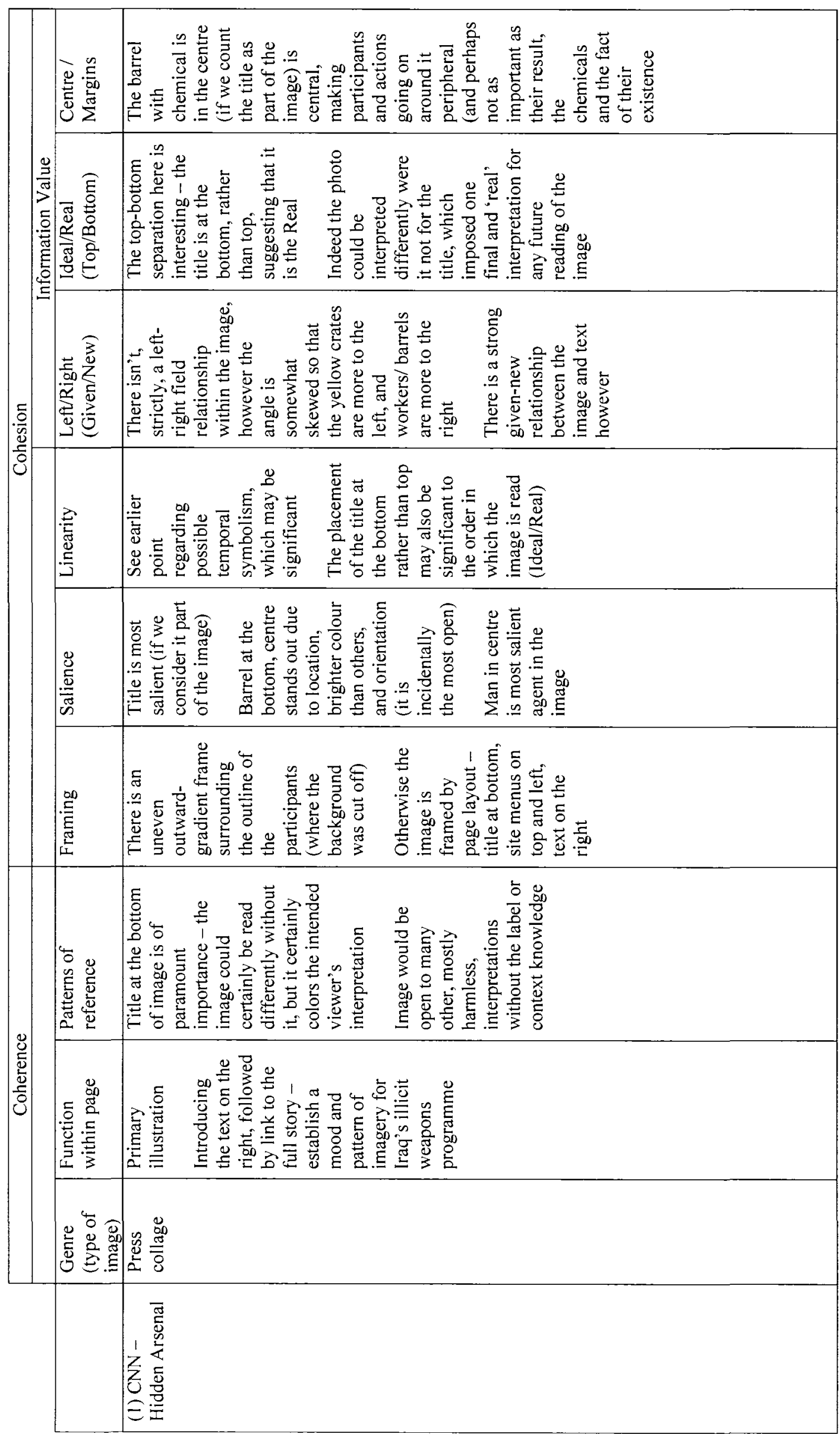




\begin{tabular}{|c|c|}
\hline 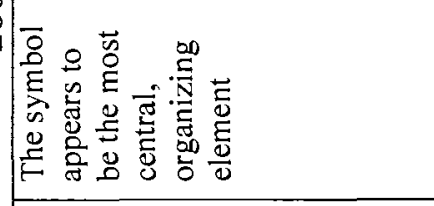 & 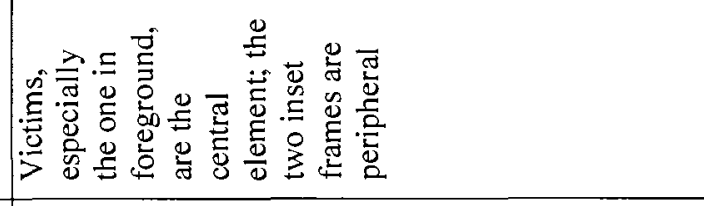 \\
\hline 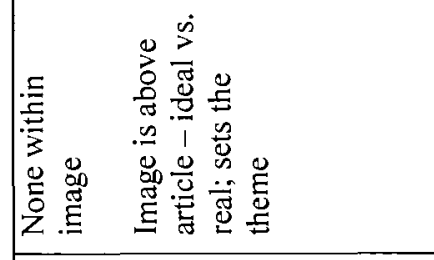 & 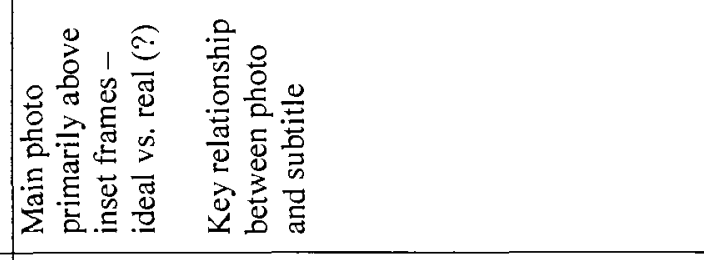 \\
\hline 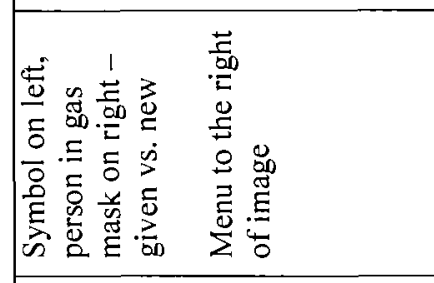 & 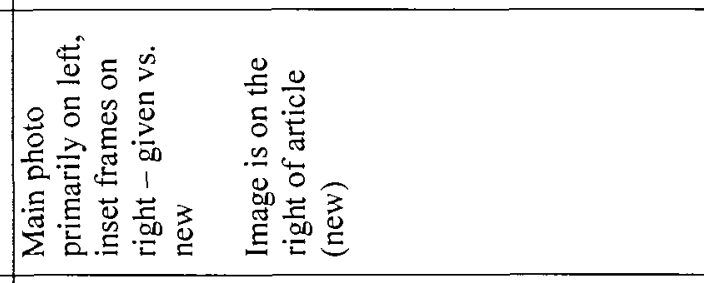 \\
\hline 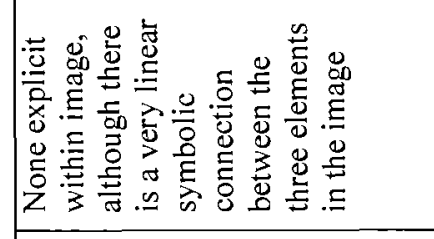 & 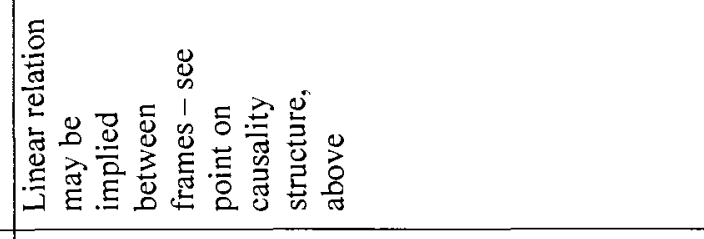 \\
\hline 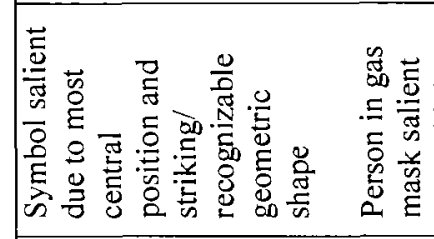 & 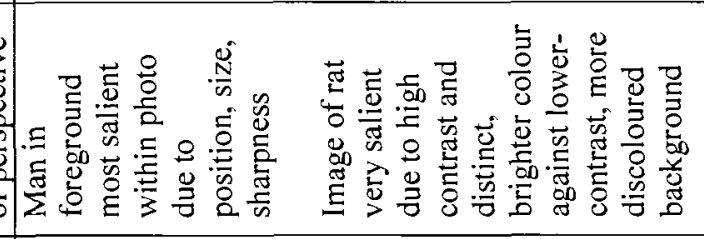 \\
\hline 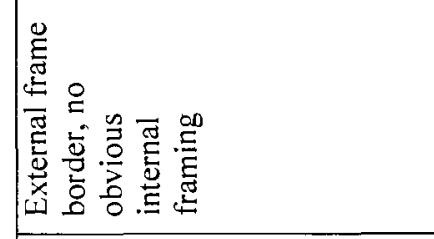 & 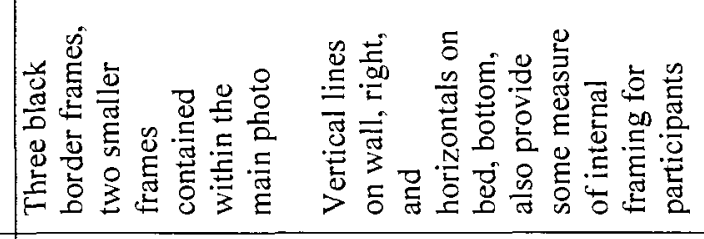 \\
\hline 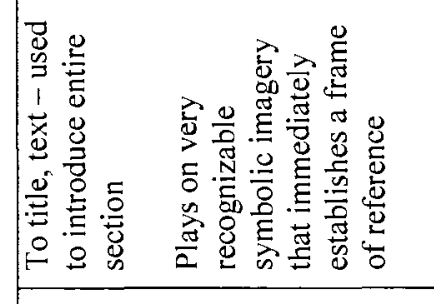 & 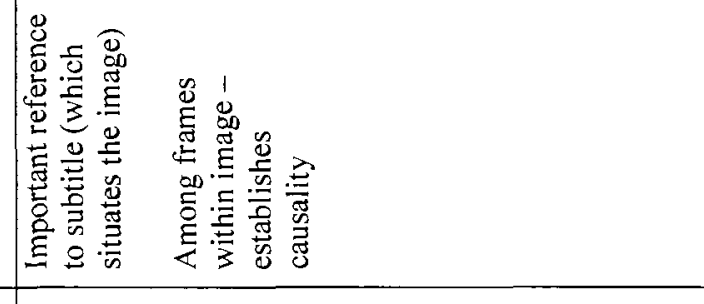 \\
\hline 亭 & 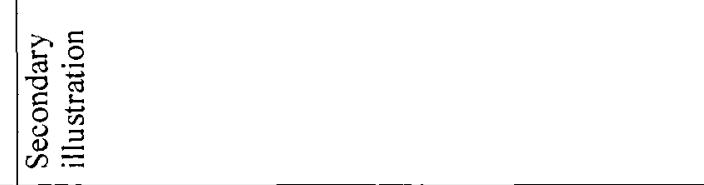 \\
\hline 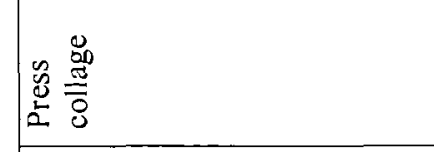 & 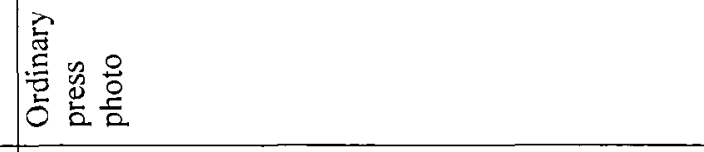 \\
\hline 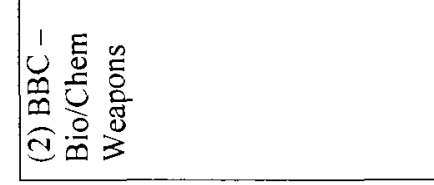 & 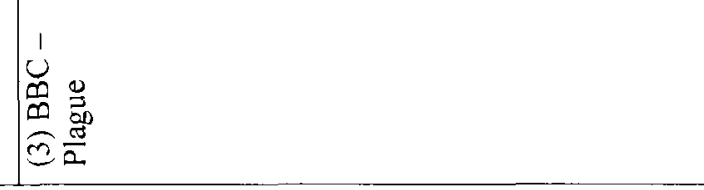 \\
\hline
\end{tabular}




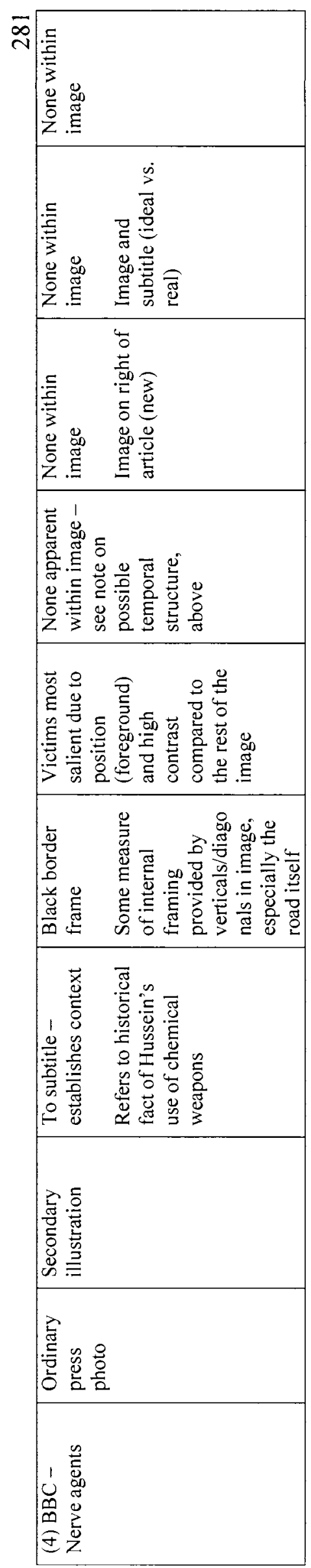

